\title{
Lewis Base Activation of Lewis Acids: Catalytic, Enantioselective Addition of Silyl Ketene Acetals to Aldehydes
}

\author{
Scott E. Denmark, Gregory L. Beutner, Thomas Wynn and Martin D. Eastgate \\ Roger Adams Laboratory, Department of Chemistry, University of Illinois \\ Urbana, Illinois 61801
}

\section{SUPPORTING INFORMATION}

\section{General Experimental}

All reactions were performed in oven-dried $\left(140^{\circ} \mathrm{C}\right)$ or flame-dried glassware under an atmosphere of dry $\mathrm{N}_{2}$. All temperatures refer to bath temperatures unless otherwise noted. Reaction solvents including dichloromethane (Fisher, HPLC Grade), diethyl ether (Fisher, BHT stabilizied HPLC Grade) and tetrahydrofuran (Fisher, HPLC Grade) were dried by percolation through two columns packed with neutral alumina under a positive pressure of argon. Reaction solvents including benzene (Fisher, thiophene free ACS Grade), hexane (Fisher, OPTIMA Grade) and toluene (Fisher, ACS Grade) were dried by percolation through a column packed with neutral alumina and a column packed with Q5 reactant, a supported copper catalyst for scavenging oxygen, under a positive pressure of argon. Solvents for chromatography and filtration were: hexane (Fisher, ACS Grade), ethyl acetate (technical grade, distilled from $\mathrm{K}_{2} \mathrm{CO}_{3}$ ), dichloromethane (technical grade, distilled from $\mathrm{CaCl}_{2}$ ). Column chromatography was performed using EM Science 230-400-mesh silica gel. Benzaldehyde (5a), 1-naphthaldehyde (5b), ${ }^{1}$ 4-tolualdehyde (5d), 4-anisaldehyde (5e), 4-trifluoromethylbenzaldehyde (5f), transcinnamaldehyde (5g), 2-methyl-trans-cinnamaldehyde $\quad \mathbf{( 5 f}), \quad$ crotonaldehyde $\quad(\mathbf{5 m}), \quad$ 2furfuraldehyde (5i), thiophene-2-carboxaldehyde (5l), cyclohexanecarboxaldehyde (5j), isovaleraldehyde (5o), hydrocinnamaldehyde (5k), chlorotrimethylsilane (Aldrich), 1-(tertbutyldimethylsilyloxy)-1-methoxyethene (Aldrich, 1a), methyl trimethylsilyl dimethylketene acetal (Aldrich, 3) and 2,2,6-trimethyl-1,3-dioxin-4-one (Lancaster) were freshly distilled before use. 2-Naphthaldehyde (5c) (Aldrich) was sublimed prior to use. Silicon tetrachloride (Aldrich) was heated at reflux for $24 \mathrm{~h}$ and then distilled prior to use. Hexamethylphosphoramide 
(Aldrich, HMPA) was distilled and then stored over $3 \AA$ molecular sieves prior to use. Trifluoroacetic anhydride (Aldrich), tert-butyl dimethylsilyl chloride (TBSCl, Gelest), 3,5dintrobenzoyl chloride (Aldrich), benzoyl chloride (Aldrich), ethyl 3,3-dimethyl acrylate (Aldrich), methyl tiglate (Lancaster), ethyl crotonate (Aldrich) and (E)-2-pentenoic acid (Lancaster) were used without further purification. tert-Butyl acetate, methyl propanoate (Aldrich), ethyl propanoate, tert-butyl propanoate, ethyl butyrate (Aldrich), propionic acid phenyl ester (TCI America), ethyl isovalerate (Fluka), ethyl isocaproate (Fluka) and 1,3dimethyl-3,4,5,6-tetrahydro-2(1H)-pyrimidinone (Aldrich, DMPU) were stored over $3 \AA$ molecular sieves. Diisopropylamine, diisopropylethylamine (DIPEA), triethylamine, pyridine and hexamethyldisilazane were freshly distilled from $\mathrm{CaH}_{2}$ prior to use. Tetrabutylammonium iodide (Aldrich) was recrystallized from toluene and dried under vacuum prior to use.

${ }^{1} \mathrm{H}$ NMR, ${ }^{13} \mathrm{C} \mathrm{NMR},{ }^{19} \mathrm{~F}$ NMR, ${ }^{31} \mathrm{P}$ NMR, ${ }^{29} \mathrm{Si}$ NMR and all low temperature spectra were recorded on a Varian Unity $400\left(400 \mathrm{MHz},{ }^{1} \mathrm{H} ; 100 \mathrm{MHz},{ }^{13} \mathrm{C}, 376 \mathrm{MHz},{ }^{19} \mathrm{~F} ; 376.288{ }^{31} \mathrm{P}\right)$, Unity $500\left(500 \mathrm{MHz},{ }^{1} \mathrm{H} ; 126 \mathrm{MHz},{ }^{13} \mathrm{C}, 202 \mathrm{MHz}\right)$ and Unity $600\left(99.443 \mathrm{MHz},{ }^{29} \mathrm{Si}\right)$ spectrometers. Spectra were referenced to residual chloroform $\left(7.26 \mathrm{ppm},{ }^{1} \mathrm{H}, 77.23 \mathrm{ppm},{ }^{13} \mathrm{C}\right)$ and ${ }^{19} \mathrm{~F}$ spectra were referenced externally to $\mathrm{C}_{6} \mathrm{~F}_{6}$. Chemical shifts are reported in ppm, multiplicities are indicated by s (singlet), d (doublet), t (triplet), q (quartet), p (pentet) h (hextet) m (multiplet) and br (broad). Coupling constants, $J$, are reported in Hertz. Mass spectroscopy was performed by the University of Illinois Mass Spectrometer Center. EI and CI mass spectra were performed on a 70-VSE spectrometer. FAB mass spectra were performed on a FinniganMATCH-5 spectrometer. Data are reported in the form of $(\mathrm{m} / \mathrm{z})$. Infrared spectra (IR) were recorded on a Mattson Galaxy 5020 spectrophotometer in $\mathrm{NaCl}$ cells. Peaks are reported in $\mathrm{cm}^{-1}$ with indicated relative intensities: s (strong, 67-100\%); m (medium, 34-66\%); w (weak, 0-33\%). Elemental analyses were performed by the University of Illinois Microanalytical Service Laboratory. Melting points ( $\mathrm{mp}$ ) were determined on a Thomas-Hoover capillary melting point apparatus in sealed tubes and are corrected. Kugelrohr distillations were performed on a Büchi GKR-50 Kugelrohr and temperatures reported are air bath temperatures (ABT). Analytical thinlayer chromatography was performed on Merck silica gel plates with QF-254 indicator. Visualization was accomplished with $\mathrm{UV}(254)$, potassium permanganate $\left(\mathrm{KMnO}_{4}\right), p$ anialdehyde and phosphomolybdic acid (PMA) staining solutions.

Optical rotation data was obtained on a JASCO DIP-360 digital polarimeter and are 
reported as follows: concentration $(\mathrm{c}=\mathrm{g} / 100 \mathrm{~mL})$, and solvent. Analytical supercritical fluid chromatography (CSP-SFC) was performed on a Berger Instruments packed-column SFC with built-in photometric detector $(220 \mathrm{~nm})$ using Daicel Chiralpak OD, OJ, OB, AD and AS columns as well as a Regis Whelk-O1 column. Analytical capillary gas chromatography (GC) was performed on a Hewlett Packard 5890 Series II fitted with a flame ionization detector $\left(\mathrm{H}_{2}\right.$ carrier gas, $16 \mathrm{~mL} / \mathrm{min}$ ) and equipped with an Astec (Chiraldex) GTA 30m x 0.25mm x 0.125 column. The injector temperature was $225^{\circ} \mathrm{C}$, the detector temperature was $300{ }^{\circ} \mathrm{C}$ Retention times $\left(t_{R}\right)$ and peak ratios were determined with a Hewlett Packard 3396 Series II integrator. Ozonolyses were performed with a Welsbach Model T-816 Ozonator set at $55 \mathrm{~W} / 90 \mathrm{~V}$ with a 1 slpm flow rate.

\section{Literature Preparations.}

$(R, R)-N, N^{\prime}$-Bis[4,5-dihydro-3,5-dimethyl-4-(3H-dinaphtho[2,1-d:1',2'-f][1,3,2]-2-oxodiazaphosphepino)]-N,N'-dimethyl-1,5-pentanediamine $((R, R)-6),{ }^{2}$ 3-phenyl-2-propynal (5n), ${ }^{3}$ (E)-(1-ethoxy-propenyloxy)-tert-butyldimethylsilane (2b), ${ }^{4} 6$-(tert-butyldimethysiloxy)hexanal $(\mathbf{5 p}),{ }^{5}$ 6-(benyloxy)hexanal $(\mathbf{5 q})^{6}$ and 6-(benzoyloxy)hexanal $(\mathbf{5 r})^{7}$ were prepared by literature methods. 


\section{Experimental Procedures.}

General Procedure 1. Enolization of Alkanoates to form Silyl Ketene Acetals.

Preparation of (E)-[(1-Methoxy)propenyloxy]-tert-butyldimethylsilane (2a)

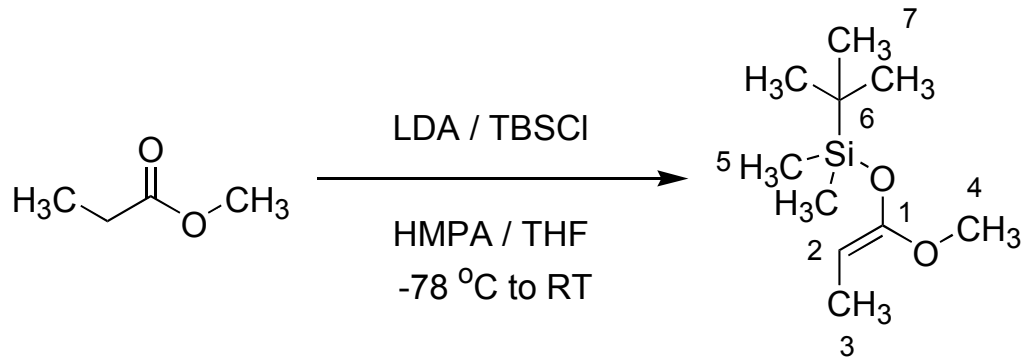

$(E)-\mathbf{2 a}$

To a flame dried, $100 \mathrm{~mL}, 3$-neck, round-bottom flask was added $1.54 \mathrm{~mL}$ of diisopropylamine (11 mmol, 1.1 equiv) and $14 \mathrm{~mL}$ of THF. The solution was cooled to $0{ }^{\circ} \mathrm{C}$ in a ice-water bath and then $9.2 \mathrm{~mL}$ of $n$-BuLi (1.2 $\mathrm{M}$ in hexane, $11 \mathrm{mmol}, 1.1$ equiv) was added slowly over five min. The resulting solution was stirred for $30 \mathrm{~min}$ at $0{ }^{\circ} \mathrm{C}$ prior to cooling to $-78{ }^{\circ} \mathrm{C}$ in a dry ice/isopropanol bath. Then $0.96 \mathrm{~mL}$ of methyl propanoate $(10 \mathrm{mmol})$ was added slowly over 5 min. The resulting solution was stirred for an additional $30 \mathrm{~min}$ at $-78{ }^{\circ} \mathrm{C}$ in a dry ice/isopropanol bath. Then a solution of $1.65 \mathrm{~g}$ of TBSCl $(11 \mathrm{mmol}, 1.1$ equiv) and $4 \mathrm{~mL}$ of HMPA (22 mmol, 2.2 equiv) in $4 \mathrm{~mL}$ of THF was added slowly via cannula. The dry ice/isopropanol bath was then removed and the solution allowed to stir for $1 \mathrm{~h}$ at room temperature. The yellow solution was diluted with cold pentane $(50 \mathrm{~mL})$ and was washed with cold water $(3 \times 75 \mathrm{~mL})$. The organic layer was dried over $\mathrm{Na}_{2} \mathrm{SO}_{4}(10 \mathrm{~g})$, and filtered through Celite and the filtrate was concentrated in vacuo. The residue was purified by Kugelrohr distillation to yield $0.852 \mathrm{~g}(42 \%, E / Z$ 82/18) of $\mathbf{2 a}$ as a colorless oil. The NMR spectral data matched that previously reported in the literature. ${ }^{8}$

Data for $(E)-\mathbf{2 a}$ :

bp: $\quad 100{ }^{\circ} \mathrm{C}(1 \mathrm{mmHg}, \mathrm{ABT})$

1 NMR: $\quad\left(500 \mathrm{MHz}, \mathrm{CDCl}_{3}\right)$

3.65 (q, $J=6.6,1 \mathrm{H}, \mathrm{HC}(2)), 3.54$ (s, $\left.3 \mathrm{H}, \mathrm{H}_{3} \mathrm{C}(4)\right), 1.49$ (d, $\left.J=6.6,3 \mathrm{H}, \mathrm{H}_{3} \mathrm{C}(3)\right)$, 0.94 (s, $\left.9 \mathrm{H}, \mathrm{H}_{3} \mathrm{C}(7)\right), 0.18$ (s, $\left.6 \mathrm{H}, \mathrm{H}_{3} \mathrm{C}(5)\right)$ 
${ }^{13} \mathrm{C}$ NMR: $\quad\left(100 \mathrm{MHz}, \mathrm{CDCl}_{3}\right)$

154.32 (C(1)), $78.83(\mathrm{C}(2)), 55.06(\mathrm{C}(4)), 25.86(\mathrm{C}(3)), 18.37(\mathrm{C}(7)), 9.67(\mathrm{C}(6))$, $-4.77(\mathrm{C}(5))$

IR: (neat)

2597 (s), 2932 (s), 2860 (s), 1686 (s), 1472 (m), 1254 (s), 1221 (s), 1177 (m), $1110(\mathrm{~m})$

MS: $\quad(\mathrm{EI}, 70 \mathrm{eV})$

$203\left(\mathrm{M}^{+1}, 31\right), 189$ (43), 163 (12), 147 (43), 131 (28), 115 (15), 89 (38), 73 (100)

Preparation of (E)-[(1-Isopropoxy)propenyloxy]tert-butyldimethylsilane (2c)

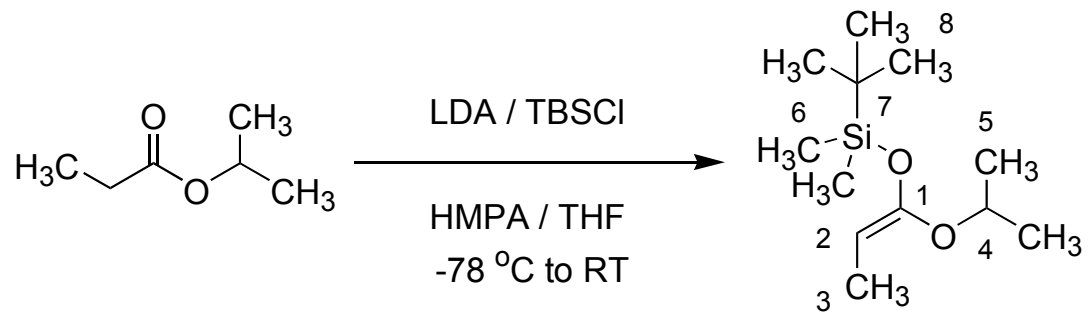

(E)-2c

Following General Procedure 1, $1.54 \mathrm{~mL}$ of diisopropylamine (11 mmol, 1.1 equiv) was combined with $6.70 \mathrm{~mL}$ of $n-\mathrm{BuLi}$ (1.64 M in hexane, $11 \mathrm{mmol}, 1.1$ equiv), $1.16 \mathrm{~g}$ of isopropyl propanoate (10 mmol, 1.0 equiv.), $1.65 \mathrm{~g}$ of TBSCl (11 mmol, 1.1 equiv) and $2.1 \mathrm{~mL}$ of HMPA (12 mmol, 1.2 equiv) to yield, after Kugelrohr distillation, $1.67 \mathrm{~g}(73 \%, E / Z 71 / 29)$ of $2 \mathrm{c}$ as a colorless oil. NMR spectral data matched that previously reported in the literature. ${ }^{9}$

Data for $(E)-\mathbf{2 c}$ :

bp: $\quad 100{ }^{\circ} \mathrm{C}(0.5 \mathrm{mmHg}, \mathrm{ABT})$

${ }^{1} \mathrm{H}$ NMR: $\quad\left(500 \mathrm{MHz}, \mathrm{CDCl}_{3}\right)$

4.33 (septet, $J=6.6,1 \mathrm{H}, \mathrm{HC}(4)$ ), 3.79 (q, $J=6.6,1 \mathrm{H}, \mathrm{HC}(2)$ ), 1.45 (d, $J=6.6$, $\left.\mathrm{H}_{3} \mathrm{C}(3)\right), 1.18\left(\mathrm{~d}, J=6.3,6 \mathrm{H}, \mathrm{H}_{3} \mathrm{C}(5)\right), 0.92$ (s, $\left.9 \mathrm{H}, \mathrm{H}_{3} \mathrm{C}(8)\right), 0.0\left(\mathrm{~s}, 6 \mathrm{H}, \mathrm{H}_{3} \mathrm{C}(6)\right)$

${ }^{13} \mathrm{C} \mathrm{NMR}: \quad\left(125 \mathrm{MHz}, \mathrm{CDCl}_{3}\right)$

$152.18(\mathrm{C}(1)), 82.01(\mathrm{C}(2)), 68.83(\mathrm{C}(4)), 25.60(\mathrm{C}(3)), 21.96(\mathrm{C}(5)), 18.08$ $(\mathrm{C}(8)), 9.79(\mathrm{C}(7)),-5.04(\mathrm{C}(6))$ 
IR: (neat)

2975 (s), 2959 (s), 2931 (s), 2841 (s), 1682 (s), 1464 (m), 1372 (m), 1362 (m),

$1340(\mathrm{~m}), 1304(\mathrm{~m}), 1254(\mathrm{~s}), 1208(\mathrm{~s}), 1110(\mathrm{~s}), 1041(\mathrm{~m})$

MS: $\quad(E I, 70 \mathrm{ev})$

$230\left(\mathrm{M}^{+}, 3\right), 187(3), 173(3), 131(51), 117(6), 75(100)$

\section{Preparation of (E)-[(1-Phenoxy)propenyloxy]-tert-butyldimethylsilane (2d)}

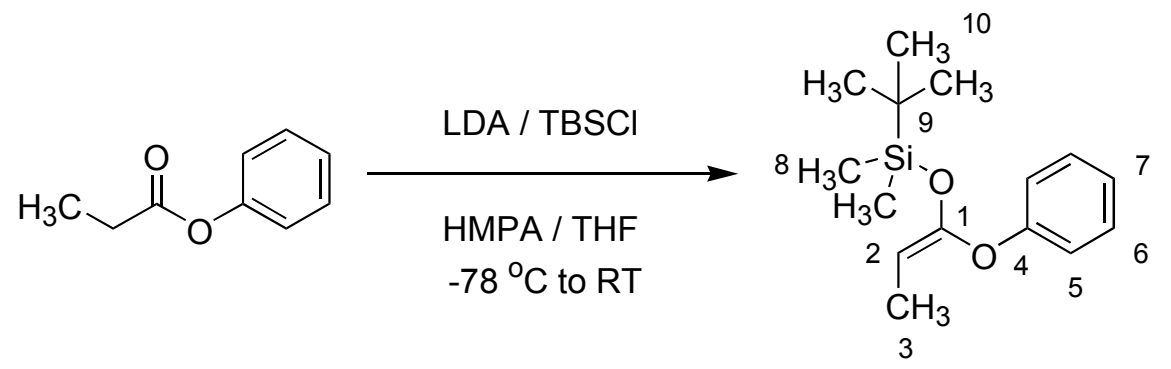

$(E)-\mathbf{2 d}$

Following General Procedure 1, $3.08 \mathrm{~mL}$ of diisopropylamine (22 mmol, 1.1 equiv) was combined with $14.0 \mathrm{~mL}$ of $n$-BuLi (1.55 M in hexane, $22 \mathrm{mmol}, 1.1$ equiv), $2.86 \mathrm{~mL}$ of propanoic acid phenyl ester ( $20 \mathrm{mmol}, 1.0$ equiv), $3.3 \mathrm{~g}$ of TBSCl ( $22 \mathrm{mmol}, 1.1$ equiv) and 8 $\mathrm{mL}$ of HMPA (44 mmol, 2.2 equiv) to yield, after Kugelrohr distillation, $2.64 \mathrm{~g} \mathrm{(50 \% ,} \mathrm{E/Z} \mathrm{94/6)}$ of $\mathbf{2 d}$ as a colorless oil.

Data for $(E)-\mathbf{2 d}$ :

bp: $\quad 125^{\circ} \mathrm{C}(1 \mathrm{mmHg}, \mathrm{ABT})$

1N NMR: $\quad\left(399 \mathrm{MHz}, \mathrm{CDCl}_{3}\right)$

7.26 (m, 2 H, HC(5)), 7.0 (m, $3 \mathrm{H}, \mathrm{HC}(6,7)), 4.26$ (q, $J=6.56,1 \mathrm{H}, \mathrm{HC}(2)), 1.52$

(d, $\left.J=6.64,3 \mathrm{H}, \mathrm{H}_{3} \mathrm{C}(3)\right), 0.819$ (s, $\left.9 \mathrm{H}, \mathrm{H}_{3} \mathrm{C}(10)\right), 0.09$ (s, $6 \mathrm{H}, \mathrm{H}_{3} \mathrm{C}(8)$ )

${ }^{13} \mathrm{C}$ NMR: $\quad\left(100 \mathrm{MHz}, \mathrm{CDCl}_{3}\right)$

$155.57(\mathrm{C}(4)), 150.18(\mathrm{C}(1)), 129.5(\mathrm{C}(6)), 122.03$ (C(7)), $116.13(\mathrm{C}(5)), 86.57$

$(\mathrm{C}(2)), 25.36(\mathrm{C}(3)), 17.91(\mathrm{C}(10)), 9.72(\mathrm{C}(9)),-5.04(\mathrm{C}(8))$

IR: (neat)

2957 (s), 2931 (s), 2887 (m), 2860 (s), 1695 (s), 1596 (s), 1491 (s), 1472 (s), 1297

(m) 
MS: $\quad(E I, 70 \mathrm{eV})$

264 (M+, 17), 207 (24), 193 (28), 171 (29), 151 (26), 137 (18), 115 (21), 105 (10), $73(100)$

HRMS: $\quad$ calcd for $\mathrm{C}_{15} \mathrm{H}_{24} \mathrm{O}_{2} \mathrm{Si}: 264.154501$, found: 264.154559

Preparation of (E)-[(1-tert-Butyloxy)propenyloxy]tert-butyldimethylsilane (2e)

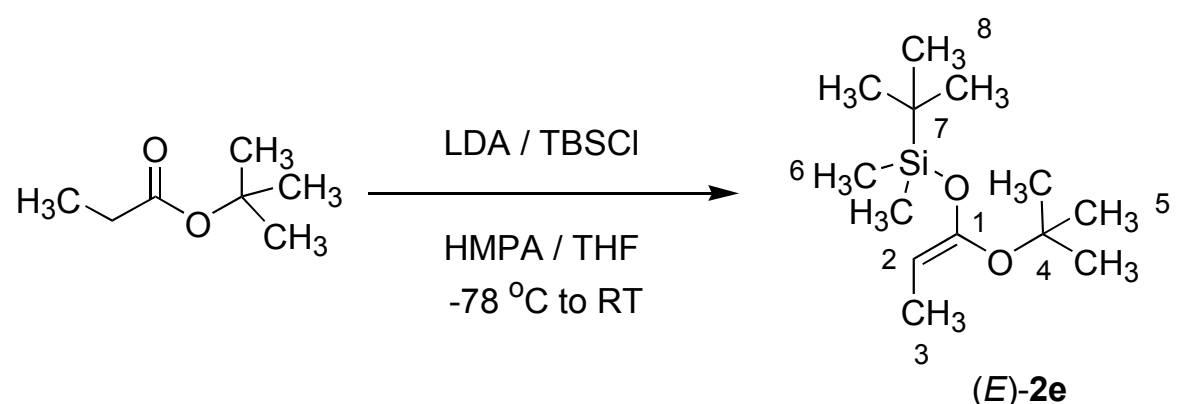

Following General Procedure 1, $6.2 \mathrm{~mL}$ of diisopropylamine (44 mmol, 1.1 equiv) was combined with $28 \mathrm{~mL}$ of $n$-BuLi (1.58 M in hexane, $44 \mathrm{mmol}, 1.1$ equiv), $6.0 \mathrm{~mL}$ of tert-butyl propanoate (40 mmol, 1.0 equiv), $6.6 \mathrm{~g}$ of TBSCl (44 mmol, 1.1 equiv) and $16 \mathrm{~mL}$ of HMPA (88 mmol, 2.2 equiv) to yield, after short path distillation, $6.8 \mathrm{~g}(70 \%, E / Z$ 95/5) of $\mathbf{2 e}$ as a colorless oil.

Data for $(E)-\mathbf{2 e :}$

bp: $\quad 70^{\circ} \mathrm{C}(1 \mathrm{mmHg}, \mathrm{ABT})$

${ }^{1} \mathrm{H}$ NMR: $\left(399 \mathrm{MHz}, \mathrm{CDCl}_{3}\right)$

$3.93(\mathrm{q}, J=6.6,1 \mathrm{H}, \mathrm{HC}(2)), 1.48\left(\mathrm{~d}, J=6.6,3 \mathrm{H}, \mathrm{H}_{3} \mathrm{C}(3)\right), 1.32(\mathrm{~s}, 9 \mathrm{H}$, $\left.\mathrm{H}_{3} \mathrm{C}(5)\right), 0.92$ (s, $\left.9 \mathrm{H}, \mathrm{H}_{3} \mathrm{C}(8)\right), 0.15$ (s, $\left.6 \mathrm{H}, \mathrm{H}_{3} \mathrm{C}(6)\right)$

${ }^{13} \mathrm{C}$ NMR: $\quad\left(100 \mathrm{MHz}, \mathrm{CDCl}_{3}\right)$

152.21 (C(1)), $86.04(\mathrm{C}(2), 78.32(\mathrm{C}(4)), 29.09(\mathrm{C}(3)), 25.74(\mathrm{C}(5)), 17.99(\mathrm{C}(8))$, $10.89(\mathrm{C}(7)),-4.81(\mathrm{C}(6))$

IR: (neat)

2997 (s), 2960 (s), 2932 (s), 2861 (s), 1678 (s), 1256 (s), 1207 (s), 1152 (s), 1110 (m), $861(\mathrm{~s}), 839$ (s), $781(\mathrm{~m})$

MS: $\quad(\mathrm{EI}, 70 \mathrm{eV})$

245 ( $\left.\mathrm{M}^{+1}, 15\right), 244$ (10), 190 (13), 189 (76), 188 (60), 187 (20), 173 (30), 132 
(14), 131 (100), 130 (11), 115 (11), 75 (90), 73 (45), 57 (55)

Anal: $\quad \mathrm{C}_{13} \mathrm{H}_{28} \mathrm{O}_{2} \mathrm{Si}(244.19)$

Calcd: $\quad$ C, $63.87 ; \quad H, 11.55 \%$

Found: $\quad$ C, 63.91; $\quad H, 11.58 \%$

Preparation of (Z)-[(1-tert-Butoxy)propenyloxy]-tert-butyldimethylsilane (2e)

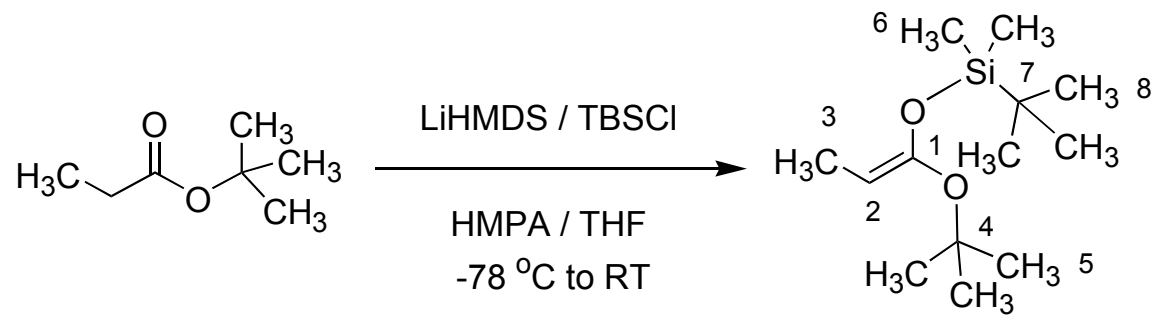

$(Z)-2 e$

To a flame dried, $250 \mathrm{~mL}$, 3-neck, round-bottom flask was added $2.46 \mathrm{~mL}$ of hexamethyldisilazane ( $11 \mathrm{mmol}, 1.0$ equiv) and $18 \mathrm{~mL}$ of THF. The solution was cooled to $0{ }^{\circ} \mathrm{C}$ under nitrogen in an ice-water bath and then $7.15 \mathrm{~mL}$ of $n$-BuLi (1.55 $\mathrm{M}$ in hexane, $11 \mathrm{mmol}, 1.1$ equiv) was added slowly over $5 \mathrm{~min}$. The resulting solution was stirred for $30 \mathrm{~min}$ at $0{ }^{\circ} \mathrm{C}$ prior to cooling to $-78{ }^{\circ} \mathrm{C}$ in a dry ice/isopropanol bath. To this solution was added $12 \mathrm{~mL}$ of HMPA ( $88 \mathrm{mmol}, 2.2$ equiv). Then $1.65 \mathrm{~mL}$ of tert-butyl propanoate $(11 \mathrm{mmol})$ was added slowly over $5 \mathrm{~min}$. The resulting solution was stirred for an additional $1 \mathrm{~h}$ at $-78{ }^{\circ} \mathrm{C}$ in a dry ice/isopropanol bath. Then a solution of $1.8 \mathrm{~g}$ of TBSCl (12 mmol, 1.1 equiv) in $8 \mathrm{~mL}$ of THF was added slowly via cannula. The dry ice/isopropanol bath was then removed and the solution allowed to stir for 1 $\mathrm{h}$ at room temperature. The yellow solution was diluted with cold pentane $(100 \mathrm{~mL})$ and washed with cold water $(3 \times 75 \mathrm{~mL})$. The organic layer was dried over $\mathrm{Na}_{2} \mathrm{SO}_{4}(10 \mathrm{~g})$, filtered through Celite and the filtrate was concentrated in vacuo. The resiude was purified by short path distillation to yield $1.72 \mathrm{~g}(64 \%, E / Z 12 / 88)$ of $2 \mathrm{e}$ as a colorless oil.

Data for $(Z)-\mathbf{2 e}:$

bp: $70{ }^{\circ} \mathrm{C}(1 \mathrm{mmHg})$

${ }^{1}$ H NMR: $\left(499 \mathrm{MHz}, \mathrm{CDCl}_{3}\right)$

$3.98(\mathrm{q}, J=6.0,1 \mathrm{H}, \mathrm{HC}(2)), 1.50(\mathrm{~d}, J=6.6,3 \mathrm{H}, \mathrm{HC}(3)), 1.28$ (s, $\left.9 \mathrm{H}, \mathrm{H}_{3} \mathrm{C}(5)\right)$, 0.95 (s, $\left.9 \mathrm{H}, \mathrm{H}_{3} \mathrm{C}(8)\right), 0.16$ (s, $\left.6 \mathrm{H}, \mathrm{H}_{3} \mathrm{C}(6)\right)$ 
${ }^{13} \mathrm{C}$ NMR: $\quad\left(125 \mathrm{MHz}, \mathrm{CDCl}_{3}\right)$

$151.63(\mathrm{C}(1)), 87.02$ (C(2)), 78.22 (C(4)), 29.10 (C(3)), 28.48 (C(5)), 17.99 $(\mathrm{C}(8)),-4.21(\mathrm{C}(6))$

IR: (neat)

2978 (s), 2959 (s), 2931 (s), 2860 (s), 1680 (s), 1473 (m), 1389 (m), 1366 (m), 1306 (s), 1254 (s), 1209 (m), 1146 (s), 1104 (m), 1049 (s)

\section{Preparation of 1-[(tert-Butoxy)ethenyloxy]tert-butyldimethylsilane (1b)}

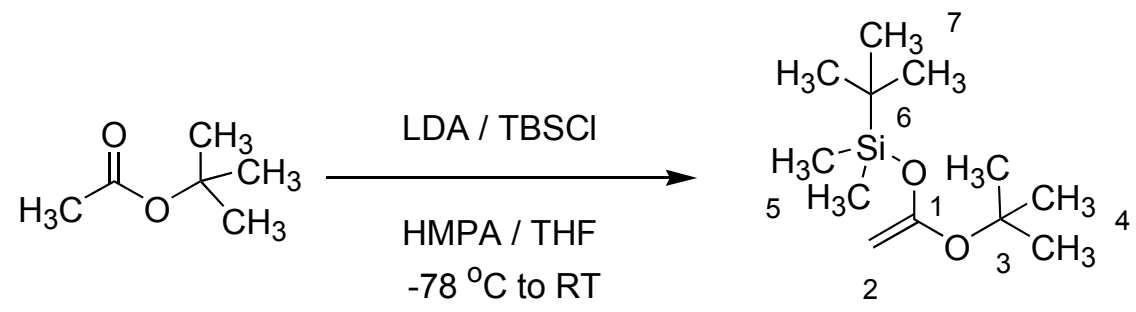

$1 b$

Following General Procedure 1, $8.1 \mathrm{~mL}$ of diisopropylamine (58 mmol, 1.1 equiv) was combined with $36.1 \mathrm{~mL}$ of $n$-BuLi (1.6 M in hexanes, $58 \mathrm{mmol}, 1.1$ equiv), $7.1 \mathrm{~mL}$ of tert-butyl acetate (53 mmol), $8.72 \mathrm{~g}$ of TBSCl (58 mmol, 1.1 equiv) and $10.1 \mathrm{~mL}$ of HMPA (58 mmol, 1.1 equiv) to yield, after short path distillation, $9.11 \mathrm{~g}(75 \%)$ of $\mathbf{1 b}$ as a colorless oil. The NMR spectral data matched that previously reported in the literature. ${ }^{9}$

Data for 1b:

bp: $\quad 66{ }^{\circ} \mathrm{C}(5.0 \mathrm{mmHg}, \mathrm{ABT})$

${ }^{1} \mathrm{H} \mathrm{NMR}: \quad\left(500 \mathrm{MHz}, \mathrm{CDCl}_{3}\right)$

3.49 (m, $1 \mathrm{H}, \mathrm{HC}(2)), 3.44$ (m, $1 \mathrm{H}, \mathrm{HC}(2)), 1.38$ (s, 9 H, HC(4)), 0.95 (s, 9 H, $\mathrm{HC}(7)), 0.20$ (s, $6 \mathrm{H}, \mathrm{HC}(5))$ 


\section{Preparation of (E)-tert-Butyl(1-ethoxy-1-butenyloxy)dimethylsilane (10a)}

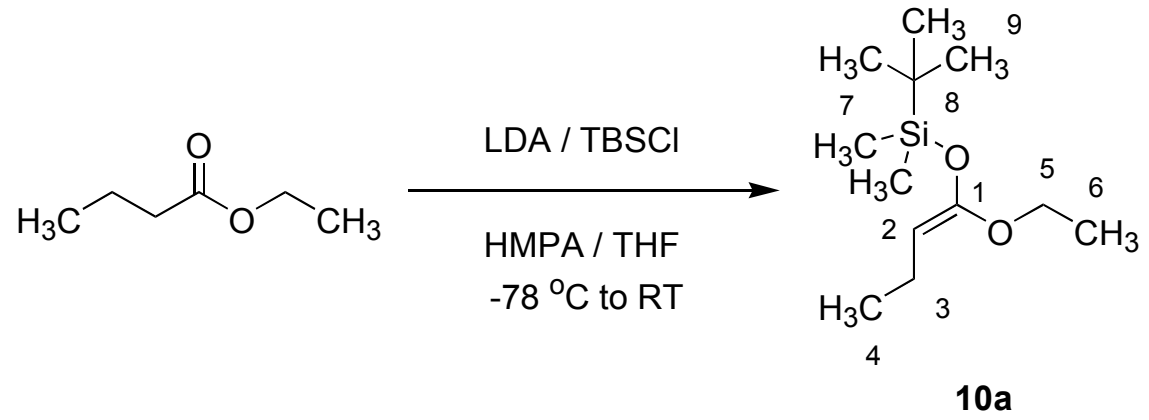

Following General Procedure 1, $1.54 \mathrm{~mL}$ of diisopropylamine (11 mmol, 1.1 equiv) was combined with $7.15 \mathrm{~mL}$ of $n$-BuLi (1.55 M in hexane, $11 \mathrm{mmol}, 1.1$ equiv), $1.32 \mathrm{~mL}$ of ethyl butyrate (10 mmol), $1.65 \mathrm{~g}$ of TBSCl (11 mmol, 1.1 equiv) and $2.1 \mathrm{~mL}$ of HMPA (12 mmol, 1.2 equiv) to yield, after short path distillation, $1.46 \mathrm{~g}(64 \%, E / Z$ 82/18) of 10a as a colorless oil.

Data for $(E)-\mathbf{1 0 a}$ :

bp: $\quad 62{ }^{\circ} \mathrm{C}(1 \mathrm{mmHg})$

${ }^{1} \mathrm{H}$ NMR: $\quad\left(400 \mathrm{MHz}, \mathrm{CDCl}_{3}\right)$

3.84 (q, $\left.J=7.1,2 \mathrm{H}, \mathrm{H}_{2} \mathrm{C}(5)\right), 3.73$ (t, $\left.J=7.3,1 \mathrm{H}, \mathrm{HC}(2)\right), 1.96$ (pent, $J=7.6,2$

$\left.\mathrm{H}, \mathrm{H}_{2} \mathrm{C}(3)\right), 1.22$ (t, $\left.J=7.1,3 \mathrm{H}, \mathrm{H}_{3} \mathrm{C}(6)\right), 0.93$ (s, $\left.9 \mathrm{H}, \mathrm{H}_{3} \mathrm{C}(9)\right), 0.91$ (t, $J=7.6,3$

$\left.\mathrm{H}, \mathrm{H}_{3} \mathrm{C}(4)\right), 0.17$ (s, $\left.6 \mathrm{H}, \mathrm{H}_{3} \mathrm{C}(7)\right)$

${ }^{13} \mathrm{C} \mathrm{NMR}: \quad\left(100 \mathrm{MHz}, \mathrm{CDCl}_{3}\right)$

152.40 (C(1)), 88.06 (C(2)), 78.32 (C(5)), 62.98 (C(3)), 25.62 (C(6)), 25.71

(C(9)), 18.05 (C(4)), $14.83(\mathrm{C}(8)),-5.04(\mathrm{C}(7))$

IR: (neat)

3072 (w), 3050 (w), 2959 (m), 2932 (m), 2859 (m), 1681 (s), 1473 (w), 1428 (m),

1374 (m), 1299 (s), 1248 (s), 1115 (s), 1020 (w)

MS: $\quad(E I, 70 \mathrm{ev})$

$230\left(\mathrm{M}^{+}, 9\right), 215$ (8), 174 (13), 145 (76), 131 (6), 115 (8), 103 (44), 75 (100)

HRMS: calcd for $\mathrm{C}_{12} \mathrm{H}_{26} \mathrm{O}_{2} \mathrm{Si}: 230.170764$, found: 230.170209

Analysis: $\quad \mathrm{C}_{12} \mathrm{H}_{26} \mathrm{O}_{2} \mathrm{Si}(230.42)$

Calcd: $\quad$ C, 62.55; H, 11.37\%

Found; $\quad \mathrm{C}, 62.37 ; \quad \mathrm{H}, 11.38 \%$ 
Preparation of (E)-tert-Butyl(1-ethoxy-3-methyl-1-butenyloxy)dimethylsilane (10b)

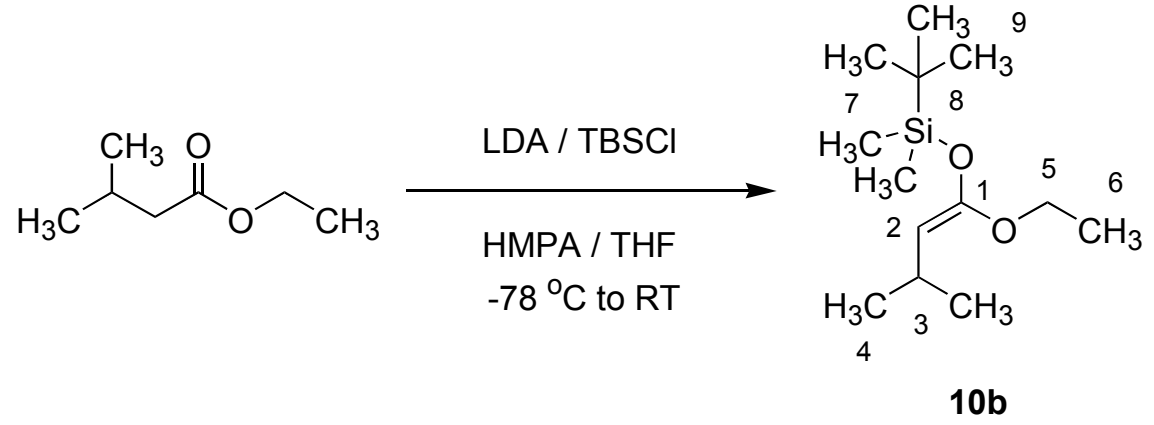

Following General Procedure 1, $1.54 \mathrm{~mL}$ of diisopropylamine (11 mmol, 1.1 equiv) was combined with $7.15 \mathrm{~mL}$ of $n$-BuLi (1.55 M in hexane, $11 \mathrm{mmol}, 1.1$ equiv), $1.5 \mathrm{~mL}$ of ethyl isovalerate (10 mmol), $1.65 \mathrm{~g}$ of TBSCl $(11 \mathrm{mmol}, 1.1$ equiv) and $2.1 \mathrm{~mL}$ of HMPA (12 mmol, 1.2 equiv) to yield, after short path distillation, $1.58 \mathrm{~g}(65 \%, E / Z 78: 22)$ of $\mathbf{1 0 b}$ as a colorless oil. Data for $(E)-\mathbf{1 0 b}$ :

bp: $\quad 62{ }^{\circ} \mathrm{C}(0.5 \mathrm{mmHg})$

${ }^{1} \mathrm{H}$ NMR: $\quad\left(400 \mathrm{MHz}, \mathrm{CDCl}_{3}\right)$

3.84 (q, $\left.J=7.1,2 \mathrm{H}, \mathrm{H}_{3} \mathrm{C}(5)\right), 3.61$ (d, $\left.J=9.0,1 \mathrm{H}, \mathrm{HC}(2)\right), 2.53$ (m, $1 \mathrm{H}, \mathrm{HC}(3)$ ),

$1.21\left(\mathrm{t}, J=7.1,3 \mathrm{H}, \mathrm{H}_{3} \mathrm{C}(6)\right), 0.93\left(\mathrm{~s}, 9 \mathrm{H}, \mathrm{H}_{3} \mathrm{C}(9)\right), 0.90$ (d, $J=6.5,6 \mathrm{H}$,

$\left.\mathrm{H}_{3} \mathrm{C}(4)\right), 0.17$ (s, $\left.6 \mathrm{H}, \mathrm{H}_{3} \mathrm{C}(7)\right)$

${ }^{13} \mathrm{C} \mathrm{NMR}: \quad\left(100 \mathrm{MHz}, \mathrm{CDCl}_{3}\right)$

$151.43(\mathrm{C}(1)), 95.15(\mathrm{C}(2)), 77.31(\mathrm{C}(5)), 62.98(\mathrm{C}(3)), 25.72(\mathrm{C}(6)), 25.63$

$(\mathrm{C}(9)), 24.09(\mathrm{C}(4)), 14.83(\mathrm{C}(8)),-5.05(\mathrm{C}(7))$

IR: (neat)

2957 (s), 2898 (m), 2862 (m), 1677 (s), $1472(\mathrm{~m}), 1464(\mathrm{~m}), 1390(\mathrm{w}), 1379(\mathrm{w})$, 1367 (w), 1275 (m), 1254 (s), 1199 (s), 1128 (s), 1099 (m), 1063 (m)

MS: (EI, $70 \mathrm{ev})$

$244\left(\mathrm{M}^{+}, 19\right), 229$ (44), 188 (17), 143 (14), 159 (38), 103 (60), 69 (100)

HRMS: calcd for $\mathrm{C}_{13} \mathrm{H}_{28} \mathrm{O}_{2} \mathrm{Si}: 244.186088$, found: 244.185859 
Preparation of (E)-tert-Butyl(1-ethoxy-4-methyl-1-pentenyloxy)]dimethylsilane (10c)
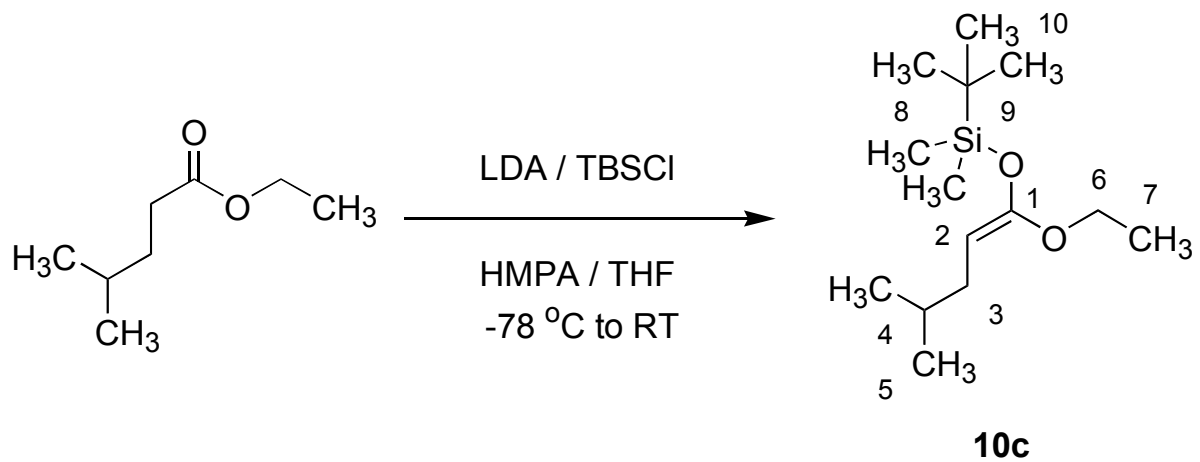

Following General Procedure 1, $1.54 \mathrm{~mL}$ of diisopropylamine (11 mmol, 1.1 equiv) was combined with $7.15 \mathrm{~mL}$ of $n$-BuLi (1.55 M in hexane, $11 \mathrm{mmol}, 1.1$ equiv), $1.66 \mathrm{~mL}$ of ethyl isocaproate (10 mmol), $1.65 \mathrm{~g}$ of TBSCl (11 mmol, 1.1 equiv) and $2.1 \mathrm{~mL}$ of HMPA (12 mmol, 1.2 equiv) to yield, after short path distillation, $1.62 \mathrm{~g}(63 \%, E / Z 84: 16)$ of $10 \mathrm{c}$ as a colorless oil. Data for $(E)-\mathbf{1 0 c}$ :

bp: $\quad 73{ }^{\circ} \mathrm{C}(1 \mathrm{mmHg})$

${ }^{1} \mathrm{H}$ NMR: $\quad\left(400 \mathrm{MHz}, \mathrm{CDCl}_{3}\right)$

3.83 (q, $\left.J=7.1,2 \mathrm{H}, \mathrm{H}_{2} \mathrm{C}(6)\right), 3.71$ (t, $\left.J=7.3,1 \mathrm{H}, \mathrm{HC}(2)\right), 1.86$ (t, $J=7.1,2 \mathrm{H}$,

$\left.\mathrm{H}_{2} \mathrm{C}(3)\right), 1.51$ (octet, $\left.J=6.6,1 \mathrm{H}, \mathrm{HC}(4)\right), 1.21$ (t, $\left.J=7.1,3 \mathrm{H}, \mathrm{H}_{3} \mathrm{C}(7)\right), 0.94$ (s, 9

$\left.\mathrm{H}, \mathrm{H}_{3} \mathrm{C}(10)\right), 0.87$ (d, $\left.J=6.6,6 \mathrm{H}, \mathrm{H}_{3} \mathrm{C}(5)\right), 0.18$ (s, $\left.6 \mathrm{H}, \mathrm{H}_{3} \mathrm{C}(8)\right)$

${ }^{13} \mathrm{C} \mathrm{NMR}: \quad\left(100 \mathrm{MHz}, \mathrm{CDCl}_{3}\right)$

$153.08(\mathrm{C}(1)), 85.12$ (C(2)), $74.72(\mathrm{C}(6)), 62.87(\mathrm{C}(3)), 33.80(\mathrm{C}(4)), 29.22$

(C(7)), 25.63 (C(10)), 22.28 (C(5)), 14.88 (C(9)), -4.98 (C(8))

IR: (neat)

2956 (s), 2932 (s), 2897 (m), 2862 (m), 1679 (s), 1471 (m), 1464 (m), 1367 (m),

1252 (s), $1198(\mathrm{~s}), 1133$ (m), 1096 (m)

MS: (EI, $70 \mathrm{ev})$

$258\left(\mathrm{M}^{+}, 28\right), 215(96), 173$ (5), 129 (13), $115(14), 103$ (23), 73 (100)

HRMS: $\quad$ calcd for $\mathrm{C}_{14} \mathrm{H}_{30} \mathrm{O}_{2} \mathrm{Si}$ : 258.201846, found: 258.201509

Analysis: $\quad \mathrm{C}_{14} \mathrm{H}_{30} \mathrm{O}_{2} \mathrm{Si}(258.47)$

Calcd: $\quad$ C, 65.06; H, 11.70\%

Found; $\quad \mathrm{C}, 64.83 ; \quad \mathrm{H}, 11.82 \%$ 


\section{General Procedure 2. Enolization of Alkenoates to Silyl Dienol Ethers.}

\section{Preparation of (Z)-tert-Butyl(1-ethoxy-1,3-butadienyloxy)dimethylsilane (4a)}

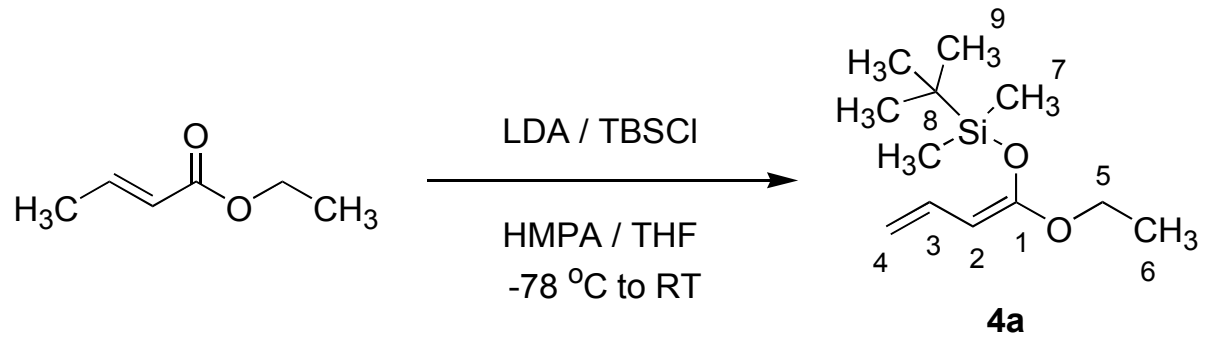

To a flame-dried, $100 \mathrm{~mL}, 3$-neck round-bottom flask was added $1.54 \mathrm{~mL}$ of diisopropylamine (11 mmol, 1.1 equiv) and $11 \mathrm{~mL}$ of THF. The solution was cooled to $0{ }^{\circ} \mathrm{C}$ in an ice-water bath and then $7.1 \mathrm{~mL}$ of $n$-BuLi (1.55 $\mathrm{M}$ in hexane, $11 \mathrm{mmol}, 1.1$ equiv) was added slowly over 5 min. The resulting solution was stirred for $30 \mathrm{~min}$ prior to cooling to $-78{ }^{\circ} \mathrm{C}$ in a dry ice/isopropanol bath. To this solution was added $2.1 \mathrm{~mL}$ of HMPA (12 mmol, 1.2 equiv). The solution was stirred for $30 \mathrm{~min}$ at $-78{ }^{\circ} \mathrm{C}$ in a dry ice/isopropanol bath and a white precipitate formed. Then, $1.24 \mathrm{~mL}$ of ethyl crotonate $(10 \mathrm{mmol})$ was added slowly over $5 \mathrm{~min}$. The resulting solution was stirred for $30 \mathrm{~min}$ at $-78^{\circ} \mathrm{C}$ prior to slow addition of $1.65 \mathrm{~g}$ of TBSCl (11.6 mmol, 1.2 equiv) in $6 \mathrm{~mL}$ of THF via cannula. The dry ice/isopropanol bath was then removed and the solution was allowed to stir for $1 \mathrm{~h}$ at room temperature. The yellow solution was diluted with cold pentane $(50 \mathrm{~mL})$ and washed with cold $\mathrm{H}_{2} \mathrm{O}(3 \times 50 \mathrm{~mL})$. The organic layer was dried over $\mathrm{Na}_{2} \mathrm{SO}_{4}(10 \mathrm{~g})$, filtered through a pad of Celite and the filtrate was concentrated in vacuo. The residue was purified by short path distillation to yield $1.63 \mathrm{~g}(71 \%, Z / E$ 86/14) of $\mathbf{4 a}$ as a colorless oil.

Data for (Z)-4a:

bp: $\quad 70{ }^{\circ} \mathrm{C}(1 \mathrm{mmHg})$

개 NMR: $\quad\left(500 \mathrm{MHz}, \mathrm{CDCl}_{3}\right)$

$6.53(\mathrm{dt}, J=17.1,10.5,1 \mathrm{H}, \mathrm{HC}(3)), 4.81(\mathrm{dd}, J=17.5,1.9,1 \mathrm{H}, \mathrm{HC}(4)), 4.57$ (dd, $J=10.7,1.5,1 \mathrm{H}, \mathrm{HC}(4)), 4.43(\mathrm{~d}, J=10.5,1 \mathrm{H}, \mathrm{HC}(2)), 3.79$ (q, $J=7.1,2$ $\mathrm{H}, \mathrm{HC}(5)), 1.30$ (t, $\left.J=7.1,3 \mathrm{H}, \mathrm{H}_{3} \mathrm{C}(6)\right), 0.95$ (s, $\left.9 \mathrm{H}, \mathrm{H}_{3} \mathrm{C}(9)\right), 0.19$ (s, $6 \mathrm{H}$, $\left.\mathrm{H}_{3} \mathrm{C}(7)\right)$ 
NOE ${ }^{1} \mathrm{H}$ NMR: $\left(500 \mathrm{MHz}, \mathrm{CDCl}_{3}\right)$

Irradiation at $3.79 \mathrm{ppm}(\mathrm{HC}(5))$ enhanced signal at $4.43 \mathrm{ppm}(\mathrm{HC}(2))$ in major isomer

${ }^{13} \mathrm{C}$ NMR: $\quad\left(100 \mathrm{MHz}, \mathrm{CDCl}_{3}\right)$

$157.73(\mathrm{C}(1)), 132.58(\mathrm{C}(3)), 106.26(\mathrm{C}(4)), 80.52(\mathrm{C}(5)), 63.26(\mathrm{C}(2)), 25.69$

$(\mathrm{C}(9)), 25.52(\mathrm{C}(6)), 14.35(\mathrm{C}(8)),-4.15(\mathrm{C}(7))$

IR: (neat)

$3085(\mathrm{w}), 2958$ (m), 2933 (m), 2888 (m), 2859 (m), $1648(\mathrm{~s}), 1602(\mathrm{w}), 1473(\mathrm{w})$, 1425 (m), 1369 (m), 1334 (m), 1255 (m), 1214 (s), 1176 (m), 1079 (m), $1025(\mathrm{~m})$

MS: $\quad(\mathrm{CI}, 70 \mathrm{eV})$

229 (M+27), 205 (26), 187 (36), 171 (36), 147 (30), 115 (52), 75 (100)

HRMS: calcd for $\mathrm{C}_{12} \mathrm{H}_{24} \mathrm{O}_{2} \mathrm{Si}$ : 229.126413, found: 229.1249

\section{Preparation of (Z)-tert-Butyl(1-methoxy-2-methyl-1,3-butadienyloxy)dimethylsilane (4b)}<smiles>CC=C(C)C(=O)OC</smiles>
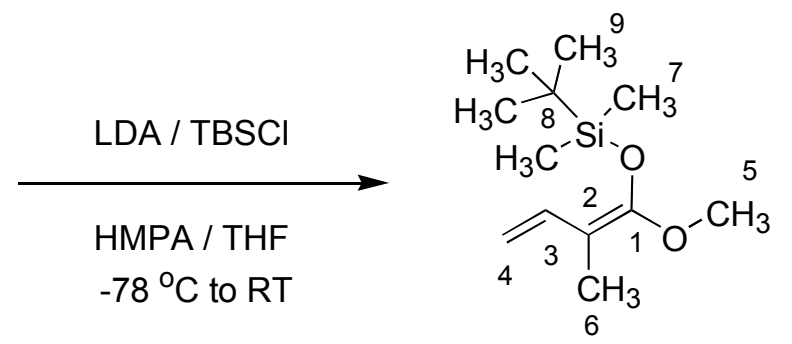

4b

Following General Procedure 2, $1.54 \mathrm{~mL}$ of diisopropylamine (11 mmol,1.1 equiv) was combined with $7.1 \mathrm{~mL}$ of $n-\mathrm{BuLi}$ (1.55 M in hexane, $11 \mathrm{mmol}, 1.1$ equiv), $2.1 \mathrm{~mL}$ of HMPA (12 mmol, 1.2 equiv), $1.2 \mathrm{~mL}$ of methyl tiglate $(10 \mathrm{mmol})$ and $1.65 \mathrm{~g}$ of TBSCl (11.6 mmol, 1.2 equiv) to yield, after short path distillation, $1.48 \mathrm{~g} \mathrm{(65 \% ,Z/E} \mathrm{58:42)} \mathrm{of} \mathbf{4 b}$ as a colorless oil. NMR spectral data matched that previously reported in the literature. ${ }^{10}$

Data for (Z)-4b:

bp: $\quad 66{ }^{\circ} \mathrm{C}(1 \mathrm{mmHg})$

근 $\quad\left(500 \mathrm{MHz}, \mathrm{CDCl}_{3}\right)$

$6.76(\mathrm{dd}, J=17.6,11.0,1 \mathrm{H}, \mathrm{HC}(3)), 4.85$ (dd, $J=17.6,1.5,1 \mathrm{H}, \mathrm{HC}(4)), 4.78$ (dd, $J=10.5,1.5,1 \mathrm{H}, \mathrm{HC}(4)), 3.58$ (s, $\left.3 \mathrm{H} \mathrm{H}_{3} \mathrm{C}(5)\right), 1.63$ (s, $\left.3 \mathrm{H}, \mathrm{H}_{3} \mathrm{C}(6)\right), 0.97$ (s, $\left.9 \mathrm{H}, \mathrm{H}_{3} \mathrm{C}(9)\right), 0.18\left(\mathrm{~s}, 6 \mathrm{H}, \mathrm{H}_{3} \mathrm{C}(7)\right)$ 
${ }^{13} \mathrm{C}$ NMR: $\quad\left(100 \mathrm{MHz}, \mathrm{CDCl}_{3}\right)$

$153.20(\mathrm{C}(1)), 134.54(\mathrm{C}(3)), 107.55(\mathrm{C}(4)), 97.43(\mathrm{C}(2)), 57.83(\mathrm{C}(5)), 25.62$ $(\mathrm{C}(9)), 18.15(\mathrm{C}(6)), 10.65(\mathrm{C}(8)),-4.49(\mathrm{C}(7))$

IR: (neat)

3089 (w), 2958 (s), 2935 (s), 2896 (m), 2859 (s), 1658 (s), 1463 (m), 1440 (m), 1290 (s), 1255 (s), 1209 (s), 1170 (s), 1124 (s), 1043 (m)

MS: $\quad(\mathrm{CI}, 70 \mathrm{eV})$

$229\left(\mathrm{M}^{+}, 6\right), 213(4), 187(100), 173(12)$

HRMS: calcd for $\mathrm{C}_{12} \mathrm{H}_{24} \mathrm{O}_{2} \mathrm{Si}: 229.126413$, found: 229.1264

\section{Preparation of (Z)-tert-Butyl(1-ethoxy-3-methyl-1,3-butadienyloxy)dimethylsilane (4c)}

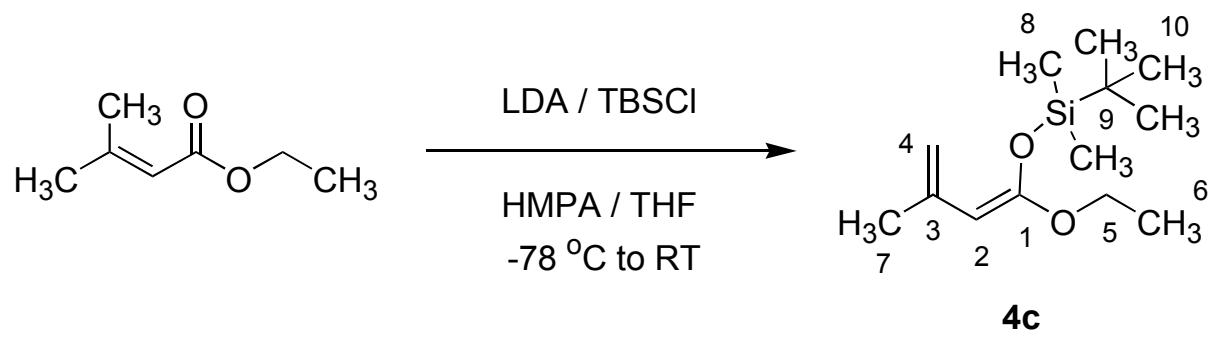

Following General Procedure 2, $1.54 \mathrm{~mL}$ of diisopropylamine (11 mmol, 1.1 equiv) was combined with $7.1 \mathrm{~mL}$ of $n$-BuLi (1.55 M in hexane, $11 \mathrm{mmol}, 1.1$ equiv), $2.1 \mathrm{~mL}$ of HMPA (12 mmol, 1.2 equiv), $1.39 \mathrm{~mL}$ of ethyl senecioate $(10 \mathrm{mmol})$ and $1.65 \mathrm{~g}$ of TBSCl (11.6 mmol, 1.2 equiv) to yield, after short path distillation, $1.65 \mathrm{~g}(68 \%, Z / E>99 / 1)$ of $4 \mathbf{c}$ as a colorless oil.

Data for $(Z)-\mathbf{4 c}$ :

bp: $\quad 68{ }^{\circ} \mathrm{C}(1 \mathrm{mmHg})$

개 NMR: $\quad\left(400 \mathrm{MHz}, \mathrm{CDCl}_{3}\right)$

4.77 (d, $J=2.4,1 \mathrm{H}, \mathrm{HC}(4)), 4.51$ (d, $J=2.4,1 \mathrm{H}, \mathrm{HC}(4)), 4.2$ (s, $1 \mathrm{H}, \mathrm{HC}(2))$,

3.78 (q, $\left.J=6.8,2 \mathrm{H}, \mathrm{H}_{2} \mathrm{C}(5)\right), 1.95$ (s, $\left.3 \mathrm{H}, \mathrm{H}_{3} \mathrm{C}(7)\right), 1.29$ (t, $J=7.1,3 \mathrm{H}$, $\left.\mathrm{H}_{3} \mathrm{C}(6)\right), 0.94$ (s, $\left.9 \mathrm{H}, \mathrm{H}_{3} \mathrm{C}(10)\right), 0.20$ (s, $6 \mathrm{H}, \mathrm{H}_{3} \mathrm{C}(8)$ )

${ }^{13} \mathrm{C} \mathrm{NMR}: \quad\left(100 \mathrm{MHz}, \mathrm{CDCl}_{3}\right)$

$156.62(\mathrm{C}(1)), 140.50(\mathrm{C}(3)), 107.02(\mathrm{C}(4)), 80.39(\mathrm{C}(4)), 63.42(\mathrm{C}(2)), 25.85$ $(\mathrm{C}(10)), 23.96(\mathrm{C}(6)), 18.14(\mathrm{C}(7)), 14.37(\mathrm{C}(9)),-3.70(\mathrm{C}(8))$ 
IR: (neat)

$3079(\mathrm{w}), 2960$ (m), 2931 (s), 2859 (m), 1650 (s), 1606 (w), 1473 (m), 1452 (m), 1349(m), 1375 (m), 1275 (m), 1195 (s), 1087 (s), 1031 (m)

MS: $\quad(\mathrm{CI}, 70 \mathrm{eV})$

$243\left(\mathrm{M}^{+}, 41\right), 201$ (73), 185 (46), 147 (38), 127 (29), 103 (70), 75 (100)

HRMS: calcd for $\mathrm{C}_{13} \mathrm{H}_{26} \mathrm{O}_{2} \mathrm{Si}$ : 243.140368, found: 243.14034

\section{Preparation of (2E)-2-Pentenoic Acid tert-Butyl Ester (30)}

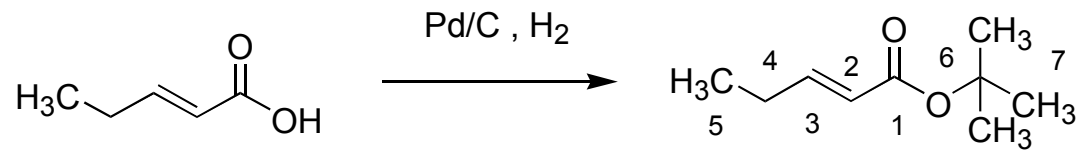

30

A $250 \mathrm{~mL}$ Parr flask containing a solution of $22.7 \mathrm{~mL}$ of trans-2-pentenoic acid $(0.225$ $\mathrm{mol})$ and $2.25 \mathrm{~mL}$ of conc. sulfuric acid ( $0.04 \mathrm{~mol}, 0.17$ equiv) in $10 \mathrm{~mL}$ of $\mathrm{Et}_{2} \mathrm{O}$ was cooled to $78{ }^{\circ} \mathrm{C}$ in a dry ice/isopropanol bath before condensation of $\sim 30 \mathrm{~mL}$ of isobutylene $(0.34 \mathrm{mmol}$, 1.5 equiv) into the solution. The flask was then placed in a sealed Parr shaker and it was shaken for $24 \mathrm{~h}$. The flask was vented and the excess isobutylene was removed by sparging the solution with an $\mathrm{N}_{2}$ stream. The solution was diluted with $50 \mathrm{~mL}$ of $\mathrm{Et}_{2} \mathrm{O}$, washed with sat. aq. $\mathrm{NaHCO}_{3}$ solution $(50 \mathrm{~mL})$, brine $(50 \mathrm{~mL})$, dried over $\mathrm{K}_{2} \mathrm{CO}_{3}(10 \mathrm{~g})$, filtered and the filtrate was concentrated by distillation of the remaining $\mathrm{Et}_{2} \mathrm{O}$ at atmospheric pressure. The residue was then purified by short path distillation to yield $8.94 \mathrm{~g}(25 \%)$ of $(2 E)-30$ as a colorless oil. NMR spectral data matched that reported in the literature. ${ }^{11}$

Data for (2E)-30:

bp: $\quad 72{ }^{\circ} \mathrm{C}(32 \mathrm{mmHg})$

근 $\quad\left(400 \mathrm{MHz}, \mathrm{CDCl}_{3}\right)$

$6.90(\mathrm{dt}, J=15.1,6.3,1 \mathrm{H}, \mathrm{HC}(3)), 5.74$ (dt, $J=15.1,1.7,1 \mathrm{H}, \mathrm{HC}(2)), 2.18$ (dpent, $\left.J=1.4,7.6,2 \mathrm{H}, \mathrm{H}_{2} \mathrm{C}(4)\right), 1.47$ (s, $\left.9 \mathrm{H}, \mathrm{H}_{3} \mathrm{C}(7)\right), 1.04$ (t, $J=7.6,3 \mathrm{H}$, $\left.\mathrm{H}_{3} \mathrm{C}(5)\right)$

${ }^{13} \mathrm{C}$ NMR: $\quad\left(100 \mathrm{MHz}, \mathrm{CDCl}_{3}\right)$

166.26 (C(1)), 149.38 (C(3)), 122.03 (C(2)), 79.95 (C(6)), 28.12 (C(4)), 25.10 (C(7)), $12.19(\mathrm{C}(5))$ 
Preparation of (1Z,3Z)-[1-(tert-Butyloxy)-1,3-pentadienyloxy]tert-butyldimethylsilane (4d)

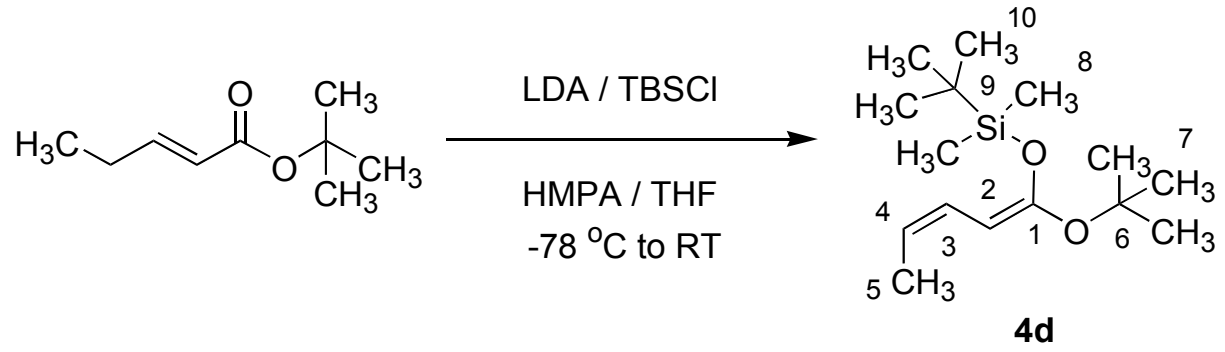

Following General Procedure 2, $1.54 \mathrm{~mL}$ of diisopropylamine (11 mmol, 1.1 equiv) was combined with $7.1 \mathrm{~mL}$ of $n-\mathrm{BuLi}$ (1.55 M in hexane, $11 \mathrm{mmol}, 1.1$ equiv), $2.1 \mathrm{~mL}$ of HMPA (12 mmol, 1.2 equiv), $1.55 \mathrm{~g}$ of tert-butyl 2-pentenoate $(10 \mathrm{mmol})$ and $1.65 \mathrm{~g}$ of TBSCl $(11.6 \mathrm{mmol}$, 1.2 equiv) to yield, after short path distillation, $1.41 \mathrm{~g}$ (52\%,1Z,3Z/others isomers 89/11) of $\mathbf{4 d}$ as a colorless oil.

Data for $(1 Z, 3 Z)-\mathbf{4 d}$ :

bp: $\quad 68{ }^{\circ} \mathrm{C}(1 \mathrm{mmHg})$

근 $\quad\left(500 \mathrm{MHz}, \mathrm{CDCl}_{3}\right)$

6.12 (dt, $J=1.7,10.7,1 \mathrm{H}, \mathrm{HC}(3)), 5.14$ (dq, $J=10.2,6.3,1 \mathrm{H}, \mathrm{HC}(4)), 4.87$ (d, $J$

$=10.8,1 \mathrm{H}, \mathrm{HC}(2)), 1.65\left(\mathrm{dd}, J=6.8,1.5,3 \mathrm{H}, \mathrm{H}_{3} \mathrm{C}(5)\right), 1.37\left(\mathrm{~s}, 9 \mathrm{H}, \mathrm{H}_{3} \mathrm{C}(7)\right)$, 0.95 (s, $\left.9 \mathrm{H}, \mathrm{H}_{3} \mathrm{C}(10)\right), 0.17$ (s, $6 \mathrm{H}, \mathrm{H}_{3} \mathrm{C}(8)$ )

NOE ${ }^{1} \mathrm{H}$ NMR: $\left(500 \mathrm{MHz}, \mathrm{CDCl}_{3}\right)$

Irradiation at $4.87 \mathrm{ppm}(\mathrm{HC}(2))$ enhanced signal at $1.65 \mathrm{ppm}(\mathrm{HC}(5))$ in major isomer

${ }^{13} \mathrm{C}$ NMR: $\quad\left(100 \mathrm{MHz}, \mathrm{CDCl}_{3}\right)$

$153.22(\mathrm{C}(1)), 124.73(\mathrm{C}(3)), 118.08(\mathrm{C}(4)), 88.38(\mathrm{C}(6)), 79.39$ (C(2)), 29.09 $(\mathrm{C}(5)), 28.50(\mathrm{C}(7)), 18.06(\mathrm{C}(10)), 13.25(\mathrm{C}(9)),-4.13(\mathrm{C}(8))$

IR: (neat)

2977 (s), 2958 (s), 2931 (s), 2859 (s), 1648 (s), 1610 (s), 1473 (m), 1367 (m), 1319 (m), 1299 (m), 1253 (s), 1201 (m), 1132 (s), 1062 (m)

MS: $\quad(\mathrm{EI}, 70 \mathrm{eV})$

$270\left(\mathrm{M}^{+}, 6\right), 231$ (5), 214 (100), 198 (42), 156 (38), 82 (98)

HRMS: calcd for $\mathrm{C}_{15} \mathrm{H}_{30} \mathrm{O}_{2} \mathrm{Si}: 270.202029$, found: 270.201509 


\section{Preparation of tert-Butyl-(2,2-dimethyl-6-methylene-6H-[1,3]dioxin-4-yloxy)dimethylsilane} (13)

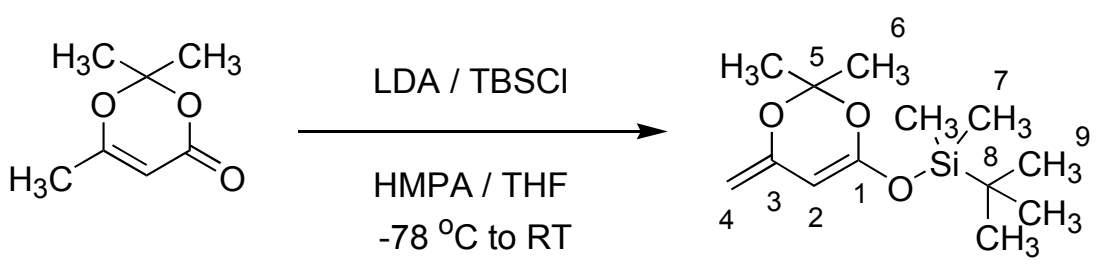

13

Following General Procedure 2, $1.54 \mathrm{~mL}$ of diisopropylamine (11 mmol, 1.1 equiv) was combined with $7.1 \mathrm{~mL}$ of $n$-BuLi (1.55 M in hexane, $11 \mathrm{mmol}, 1.1$ equiv), $2.1 \mathrm{~mL}$ of HMPA (12 mmol, 1.2 equiv), $1.3 \mathrm{~mL}$ of 2,2,6-Trimethyl-1,3-dioxin-4-one $(10 \mathrm{mmol})$ and $1.65 \mathrm{~g}$ of TBSCl (11.6 mmol, 1.2 equiv) to yield, after short path distillation, $1.52 \mathrm{~g} \mathrm{(59 \% )} \mathrm{of} \mathbf{1 3}$ as a colorless oil.

Data for 13:

bp: $\quad 78{ }^{\circ} \mathrm{C}(1 \mathrm{mmHg})$

글 NMR: $\quad\left(400 \mathrm{MHz}, \mathrm{CDCl}_{3}\right)$

4.65 (s, $1 \mathrm{H}, \mathrm{HC}(2)), 4.05$ (s, $1 \mathrm{H}, \mathrm{HC}(4)), 3.86$ (s, $1 \mathrm{H}, \mathrm{HC}(4)), 1.54$ (s, $6 \mathrm{H}$, $\mathrm{HC}(6)), 0.93$ (s, $\left.9 \mathrm{H}, \mathrm{H}_{3} \mathrm{C}(9)\right), 0.20$ (s, $6 \mathrm{H}, \mathrm{H}_{3} \mathrm{C}(7)$ )

${ }^{13} \mathrm{C} \mathrm{NMR}: \quad\left(100 \mathrm{MHz}, \mathrm{CDCl}_{3}\right)$

$153.54(\mathrm{C}(1)), 151.94(\mathrm{C}(3)), 102.45(\mathrm{C}(5)), 84.67(\mathrm{C}(4)), 76.64(\mathrm{C}(2)), 25.49$ $(\mathrm{C}(9)), 24.50(\mathrm{C}(6)), 18.01(\mathrm{C}(8)),-4.28(\mathrm{C}(7))$

IR: (neat) 2996 (m), 2958 (m), 2933 (m), 2888 (w), 2861 (m), $1668(\mathrm{~s}), 1633(\mathrm{~m}), 1473(\mathrm{w})$, 1386 (m), 1375 (m), 1344 (s), 1294 (m), 1261 (s), 1224 (s), 1205 (s), 1126 (m), $1031(\mathrm{~m})$

MS: $\quad(\mathrm{CI}, 70 \mathrm{eV})$ $257\left(\mathrm{M}^{+}, 100\right), 215$ (21), 173 (22), 143 (24), 85 (29)

HRMS: calcd for $\mathrm{C}_{13} \mathrm{H}_{24} \mathrm{O}_{3} \mathrm{Si}: 257.157488$, found: 257.157298 
General Procedure 3. Addition of Acetate-derived Silyl Ketene Acetals to Aromatic Aldehydes. Preparation of (3R)-Methyl 3-Hydroxy-3-phenylpropanoate (7aa)

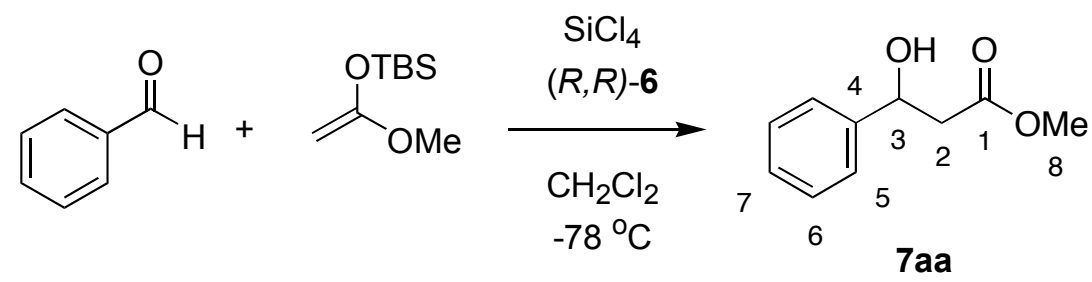

A flame-dried, 20-mL, 2-neck flask containing a solution of $84 \mathrm{mg}(0.1 \mathrm{mmol}, 0.05$ equiv) of bis-phosphoramide $((R, R)-6)$ in $\mathrm{CH}_{2} \mathrm{Cl}_{2}(10 \mathrm{~mL})$ was cooled to $-78{ }^{\circ} \mathrm{C}$ under nitrogen in a dry ice/isopropanol bath and then $200 \mu \mathrm{L}(2.0 \mathrm{mmol})$ of benzaldehyde was added in one portion. To the resulting solution $252 \mu \mathrm{L}\left(2.2 \mathrm{mmol}, 1.1\right.$ equiv) of $\mathrm{SiCl}_{4}$ was added and the reaction mixture was allowed to stir at $-78{ }^{\circ} \mathrm{C}$ for $5 \mathrm{~min}$ in a dry ice/isopropanol bath. Then $523 \mu \mathrm{L}(2.4 \mathrm{mmol}$, 1.2 equiv) of 1-(tert-butyldimethylsilyloxy)-1-methoxyethene (1a) was added dropwise (over 5 $\min$ ) to the reaction mixture. The resulting mixture was allowed to stir at $-78{ }^{\circ} \mathrm{C}$ for 15 min in a dry ice/isopropanol bath whereupon the cold reaction mixture was poured into a rapidly stirring solution of $1 / 1$ sat. aq. $\mathrm{KF} / 1.0 \mathrm{M} \mathrm{KH}_{2} \mathrm{PO}_{4}$ solution $(50 \mathrm{~mL})$. This biphasic mixture was stirred vigorously for $2 \mathrm{~h}$ after which the aqueous layer was washed with $\mathrm{CH}_{2} \mathrm{Cl}_{2}(3 \times 50 \mathrm{~mL})$. The combined organic extracts were dried over $\mathrm{Na}_{2} \mathrm{SO}_{4}$, filtered and the filtrate was concentrated in vacuo. The residue was purified by silica gel column chromatography (hexanes/EtOAc, 2/1). The product-containing fractions were combined and the solvent was removed in vacuo to yield $348 \mathrm{mg}(97 \%)$ of (3R)-methyl 3-hydroxy-3-phenylpropanoate (7aa) as a colorless oil. NMR spectral data matched that previously reported in the literature. ${ }^{12}$

Data for $(3 R)-7 \mathbf{a a}$ :

${ }^{1} \mathrm{H}$ NMR: $\quad\left(400 \mathrm{MHz}, \mathrm{CDCl}_{3}\right)$

7.40-7.34 (m, 4 H, 2 x HC(5), 2 x HC(6)), 7.31-7.27 (m, $1 \mathrm{H}, \mathrm{HC}(7)), 5.16$ (dt, $J$ $=8.8,3.4,1 \mathrm{H}, \mathrm{HC}(3)), 3.73$ (s, $3 \mathrm{H}, \mathrm{HC}(8)), 3.23$ (bs, $1 \mathrm{H}, \mathrm{HO}), 2.75$ (dd, $J=$ 16.5, 8.8, $1 \mathrm{H}, \mathrm{H} H \mathrm{C}(2)), 2.73(\mathrm{dd}, J=16.4,6.8,1 \mathrm{H}, H \mathrm{HC}(2))$

${ }^{13} \mathrm{C} \mathrm{NMR}: \quad\left(100 \mathrm{MHz}, \mathrm{CDCl}_{3}\right)$

173.03 (C(1)), 142.63 (C(4)), 128.78 (C(6)), 128.05 (C(7)), 125.84 (C(5)), 70.50 (C(3)), $52.13(\mathrm{C}(8)), 43.31(\mathrm{C}(2))$ 
IR: (neat)

3456 (s), $3031(\mathrm{~m}), 3005$ (w), $2953(\mathrm{~m}), 1735$ (s), $1494(\mathrm{w}), 1438$ (s), 1403 (s), 1360(m), 1269 (m), 1200 (m), 1164 (m), 1060 (m), 1036 (m), 1025 (m), 988 (w), $762(\mathrm{~m}), 701(\mathrm{~m})$

SFC: $\quad t_{R}(R)-7$ aa, $3.81(96.3 \%) ; t_{R}(S)-7 \mathbf{a a}, 4.23$ min (3.7\%) (Chiralpak AD, 200 psi, 40 ${ }^{\circ} \mathrm{C}, 3.5 \% \mathrm{CH}_{3} \mathrm{OH}$ in $\mathrm{CO}_{2}, 2.7 \mathrm{~mL} / \mathrm{min}, 220 \mathrm{~nm}$ )

Opt. Rot.: $\quad[\alpha]_{\mathrm{D}}^{24}+17.7(\mathrm{c}=2.05, \mathrm{EtOH})\left[\mathrm{Lit}^{12}:[\alpha]_{\mathrm{D}}^{24}(R)+12.4(\mathrm{c}=2.66, \mathrm{EtOH})\right]$

\section{Preparation of Methyl 3-Hydroxy-3-(1-naphthyl)propanoate (7ab)}

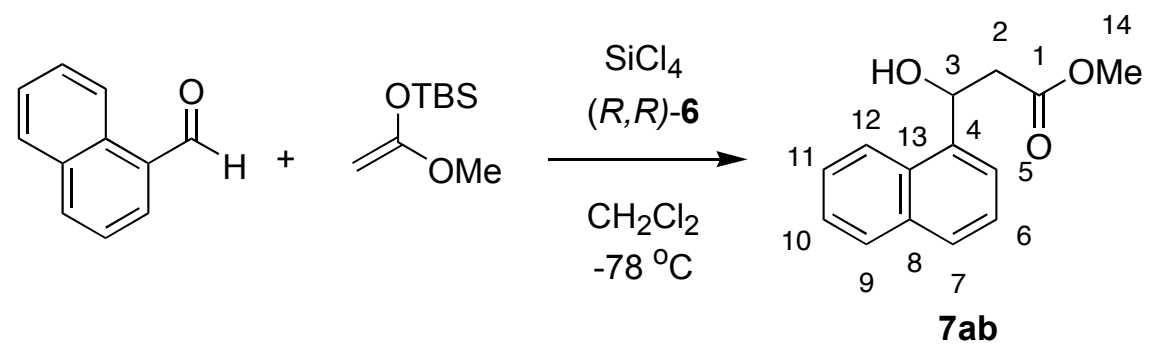

Following General Procedure 3, $271 \mu \mathrm{L}(2.0 \mathrm{mmol})$ of 1-naphthaldehyde was combined with 84 $\mathrm{mg}(0.1 \mathrm{mmol}, 0.05$ equiv) of bis-phosphoramide $((R, R)-6), 252 \mu \mathrm{L}(2.2 \mathrm{mmol}, 1.1$ equiv) of $\mathrm{SiCl}_{4}$, and $523 \mu \mathrm{L}$ (2.4 mmol, 1.2 equiv) of 1-(tert-butyldimethylsilyloxy)-1-methoxyethene (1a) to yield, after purification by silica gel column chromatography (hexanes/EtOAc, 2/1), $457 \mathrm{mg}$ (98\%) of methyl 3-hydroxy-3-(2-naphthyl) propanoate (7ab) as a colorless solid. An analytical sample was obtained by recrystallization from $\mathrm{Et}_{2} \mathrm{O}$ to afford colorless crystals.

Data for 7ab:

mp: $\quad 76-77^{\circ} \mathrm{C}\left(\mathrm{Et}_{2} \mathrm{O}\right)$

글 NMR: $\quad\left(400 \mathrm{MHz}, \mathrm{CDCl}_{3}\right)$

8.05 (d, $J=8.3,1 \mathrm{H}, \mathrm{HC}(12)), 7.89$ (d, $J=7.5,1 \mathrm{H}, \mathrm{HC}(9)), 7.80(\mathrm{~d}, J=8.1,1 \mathrm{H}$, $\mathrm{HC}(7)), 7.71(\mathrm{~d}, J=7.0,1 \mathrm{H}, \mathrm{HC}(5)), 7.51$ (m, $3 \mathrm{H}, \mathrm{HC}(6), \mathrm{HC}(10), \mathrm{HC}(11))$, 5.93 (m, 1 H, HC(3)), 3.78 (s, 3 H, HC(14)), 3.35 (bs, 1 H, HO), 2.91 (dd, $J=$ 16.3, 5.3, $1 \mathrm{H}, \mathrm{H} H \mathrm{C}(2)), 2.90(\mathrm{dd}, J=16.3,6.0,1 \mathrm{H}, H \mathrm{HC}(2))$ 
${ }^{13} \mathrm{C}$ NMR: $\quad\left(100 \mathrm{MHz}, \mathrm{CDCl}_{3}\right)$

$173.31(\mathrm{C}(1)), 138.12(\mathrm{C}(4)), 133.94(\mathrm{C}(8)), 130.10(\mathrm{C}(13)), 129.22(\mathrm{C}(9))$, $128.52(\mathrm{C}(7)), 126.51(\mathrm{C}(11)), 125.83(\mathrm{C}(10)), 125.72(\mathrm{C}(6)), 123.11(\mathrm{C}(5))$, $122.92(\mathrm{C}(12)), 67.50(\mathrm{C}(3)), 52.23(\mathrm{C}(14)), 42.70(\mathrm{C}(2))$

IR: (neat)

3459 (s), 3051 (s), 3007 (w), 2951 (m), 1731 (s), 1597 (w), 1511 (m), 1437 (s), 1356 (m), 1279 (m), 1206 (m), 1173 (s), 1083 (m), 1042 (s), 1001 (w), 906 (w), $861(\mathrm{w}), 803(\mathrm{~s}), 780(\mathrm{~s}), 737(\mathrm{w}), 695(\mathrm{w})$

MS: $\quad(E I, 70 \mathrm{eV})$

$230\left(\mathrm{M}^{+}, 31\right), 157$ (100), $129(80)$

TLC: $\quad R_{f} 0.35$ (Hexanes/EtOAc, 2/1) [UV(254)/PMA]

Opt. Rot.: $\quad[\alpha]_{\mathrm{D}}^{24}+21.00(\mathrm{c}=2.12, \mathrm{EtOH})$

SFC: $\quad t_{R}(S)-7 \mathbf{a b}, 5.17$ (9.8\%); $t_{R}(R)-7 \mathbf{a b}, 5.91 \mathrm{~min}(90.2 \%)$ (Chiralpak OD, $200 \mathrm{psi}, 40$ ${ }^{\circ} \mathrm{C}, 9.0 \% \mathrm{CH}_{3} \mathrm{OH}$ in $\left.\mathrm{CO}_{2}, 3.0 \mathrm{~mL} / \mathrm{min}, 220 \mathrm{~nm}\right)$

Analysis: $\quad \mathrm{C}_{14} \mathrm{H}_{14} \mathrm{O}_{3}(230.26)$

Calcd: $\quad$ C, $73.03 ; \quad \mathrm{H}, 6.13 \%$

Found: $\quad$ C, $72.93 ; \quad \mathrm{H}, 6.16 \%$

Preparation of Methyl 3-Hydroxy-3-(2-naphthyl)propanoate (7ac)

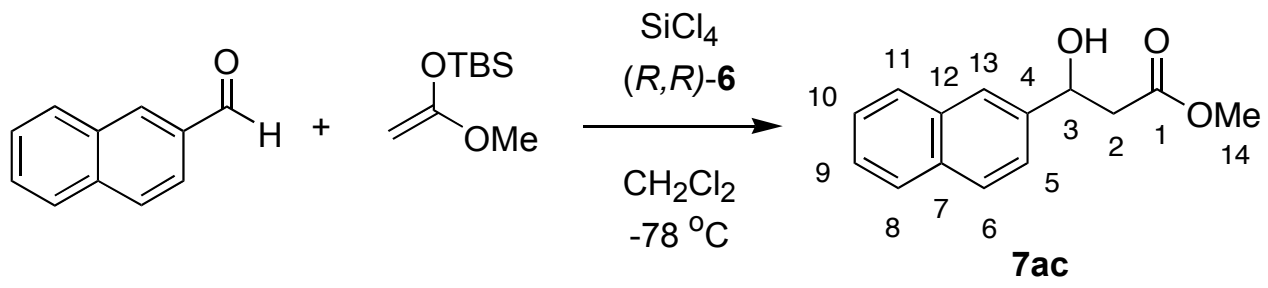

Following General Procedure 3, $312.3 \mathrm{mg}$ (2.0 mmol) of 2-naphthaldehyde was combined with $84 \mathrm{mg}$ ( $0.1 \mathrm{mmol}, 0.05$ equiv) of bis-phosphoramide $((R, R)-6), 252 \mu \mathrm{L}$ (2.2 mmol, 1.1 equiv) of $\mathrm{SiCl}_{4}$, and $523 \mu \mathrm{L}$ (2.4 mmol, 1.2 equiv) of 1-(tert-butyldimethylsilyloxy)-1-methoxyethene (1a) to yield, after purification by silica gel column chromatography (hexanes/EtOAc, 2/1), $455 \mathrm{mg}$ (98\%) of methyl 3-hydroxy-3-(2-naphthyl) propanoate (7ac) as a wax-like, white solid. After Kugelrohr distillation an analytically pure, colorless oil was isolated which solidified upon standing. 
Data for 7ac:

bp: $\quad 145^{\circ} \mathrm{C}\left(1.8 \times 10^{-4} \mathrm{mmHg}, \mathrm{ABT}\right)$

근 $\quad\left(400 \mathrm{MHz}, \mathrm{CDCl}_{3}\right)$

7.83 (m, 4 H, H(Ar)), 7.48 (m, 3 H, H(Ar)), 5.31 (m, 1 H, HC(3)), 3.74 (s, 3 H, HC(14)), 3.34 (m, $1 \mathrm{H}, \mathrm{HO}), 2.84$ (dd, $J=16.4,6.0,1 \mathrm{H}, \mathrm{H} H \mathrm{C}(2)), 2.83(\mathrm{dd}, J=$ 16.4, 2.0, $1 \mathrm{H}, \mathrm{HHC}(2))$

${ }^{13} \mathrm{C} \mathrm{NMR}: \quad\left(100 \mathrm{MHz}, \mathrm{CDCl}_{3}\right)$

$173.06(\mathrm{C}(1)), 140.03(\mathrm{C}(4)), 133.48(\mathrm{C}(12)), 133.24(\mathrm{C}(7)), 128.65(\mathrm{C}(8))$, 128.25 (C(11)), 127.90 (C(5)), 126.47 (C(6)), 126.24 (C(13)), 124.68 (C(10)), 123.89 (C(9)), 70.63 (C(3)), 52.16 (C(14)), 43.28 (C(2))

IR: (neat)

3442 (s), 3055 (s), 3022 (m), 2952 (m), 1732 (s), 1601 (w), 1509 (m), 1437 (s), 1404 (w), 1358 (m), 1274 (s), 1203 (m), 1169 (m), 1067 (m), 1034 (m), 897 (w), $821(\mathrm{~s}), 748(\mathrm{~s}), 692(\mathrm{w})$

MS: $\quad(\mathrm{EI}, 70 \mathrm{eV})$

$230\left(\mathrm{M}^{+} 41\right), 157$ (100), 129 (120)

TLC: $\quad R_{f} 0.30$ (hexanes/EtOAc, 2/1) [UV(254)/PMA]

Opt. Rot.: $\quad[\alpha]_{\mathrm{D}}^{24}+15.66(\mathrm{c}=2.11, \mathrm{EtOH})$

SFC: $\quad t_{R}(R)-7 \mathbf{a c}, 4.72(96.8 \%) ; t_{R}(S)-7 \mathbf{a c}, 5.26 \mathrm{~min}(3.2 \%)$ (Chiralpak AD, 150 psi, 40 ${ }^{\circ} \mathrm{C}, 10.0 \% \mathrm{CH}_{3} \mathrm{OH}$ in $\mathrm{CO}_{2}, 3.0 \mathrm{~mL} / \mathrm{min}, 220 \mathrm{~nm}$ )

Analysis: $\quad \mathrm{C}_{14} \mathrm{H}_{14} \mathrm{O}_{3}(230.26)$

Calcd: $\quad$ C, $73.03 ; \quad H, 6.13 \%$

Found: $\quad$ C, $72.82 ; \quad H, 6.02 \%$ 
Preparation of Methyl 3-Hydroxy-3-(4-methylphenyl)propanoate (7ad)

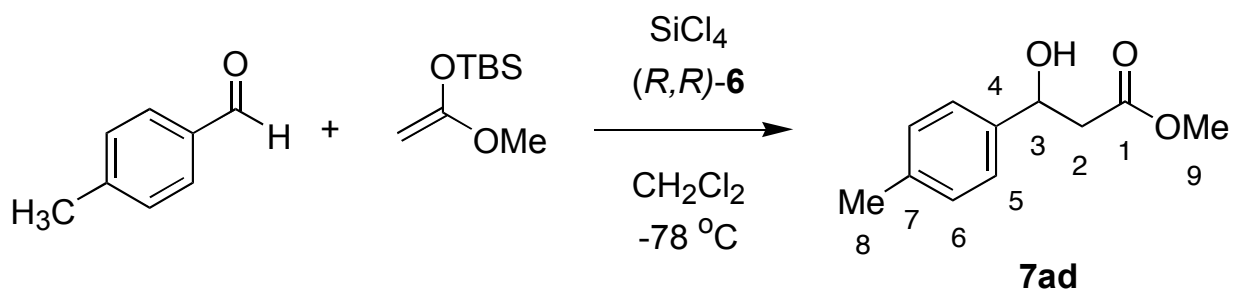

Following General Procedure 3, $236 \mu \mathrm{L}(2.0 \mathrm{mmol})$ of anisaldehyde was combined with $84 \mathrm{mg}$ (0.1 mmol, 0.05 equiv) of bis-phosphoramide $((R, R)-6), 252 \mu \mathrm{L}$ (2.2 mmol, 1.1 equiv) of $\mathrm{SiCl}_{4}$, and $523 \mu \mathrm{L}$ (2.4 mmol, 1.2 equiv) of 1-(tert-butyldimethylsilyloxy)-1-methoxyethene (1a) to yield, after purification by silica gel column chromatography (hexanes/EtOAc, 2/1), $378 \mathrm{mg}$ (97\%) of methyl 3-hydroxy-3-(4-methylphenyl) propanoate (7ad) as a clear oil. After Kugelrohr distillation an analytically pure, colorless oil was collected.

Data for 7ad:

bp: $\quad 105^{\circ} \mathrm{C}\left(1.4 \times 10^{-4} \mathrm{mmHg}, \mathrm{ABT}\right)$

1 $\mathrm{H}$ NMR: $\quad\left(400 \mathrm{MHz}, \mathrm{CDCl}_{3}\right)$

$7.28(\mathrm{~d}, J=8.1,2 \mathrm{H}, \mathrm{HC}(5)), 7.18(\mathrm{~d}, J=8.1,2 \mathrm{H}, \mathrm{HC}(6)), 5.12(\mathrm{dt}, J=9.0,3.6$, $1 \mathrm{H}, \mathrm{HC}(3)), 3.75$ (s, $3 \mathrm{H}, \mathrm{HC}(9)), 3.13$, (d, $J=3.4,1 \mathrm{H}, \mathrm{HO}$ ), 2.76 (dd, $J=16.4$, 7.8, $1 \mathrm{H}, \mathrm{H} H \mathrm{C}(2)), 2.74(\mathrm{dd}, J=16.4,5.3,1 \mathrm{H}, H \mathrm{HC}(2)), 1.60$ (s, $3 \mathrm{H}, \mathrm{HC}(8))$

${ }^{13} \mathrm{C} \mathrm{NMR}: \quad\left(100 \mathrm{MHz}, \mathrm{CDCl}_{3}\right)$ 173.08 (C(1)), $139.69(\mathrm{C}(4)), 137.80$ (C(7)), $129.46(\mathrm{C}(6)), 125.80$ (C(5)), 70.39

(C(3)), $52.13(\mathrm{C}(9)), 43.32(\mathrm{C}(2)), 21.34(\mathrm{C}(8))$

IR: (neat)

3450 (s), 3023 (w), 2952 (m), 2922 (m), 1737 (s), 1515 (m), 1438 (m), 1357 (w), 1267 (w), 1198 (m), 1162 (m), 1067 (w), 1035 (m), 1019 (w), 989 (w), 818 (m)

MS: $\quad(E I, 70 \mathrm{eV})$

$194\left(\mathrm{M}^{+}, 22\right) 179$ (10), 122 (10), 121 (100), 105 (10)

TLC: $\quad R_{f} 0.33$ (hexanes/EtOAc, 2/1) [UV(254)/PMA]

Opt. Rot.: $\quad[\alpha]_{D}^{24}+16.51(\mathrm{c}=2.21, \mathrm{EtOH})$

SFC: $\quad t_{R}(R)$-7ad, 3.97 (97.2\%); $t_{R}(S)$-7ad, 4.37 min (2.8\%) (Chiralpak AD, 150 psi, 40 ${ }^{\circ} \mathrm{C}, 4.0 \% \mathrm{CH}_{3} \mathrm{OH}$ in $\mathrm{CO}_{2}, 3.0 \mathrm{~mL} / \mathrm{min}, 220 \mathrm{~nm}$ ) 
Analysis: $\quad \mathrm{C}_{11} \mathrm{H}_{14} \mathrm{O}_{3}(194.23)$

Calcd: $\quad$ C, 68.02; H, 7.27\%

Found: $\quad$ C, 67.83; H, $7.32 \%$

Preparation of Methyl 3-Hydroxy-3-(4-methoxyphenyl)propanoate (7ae)

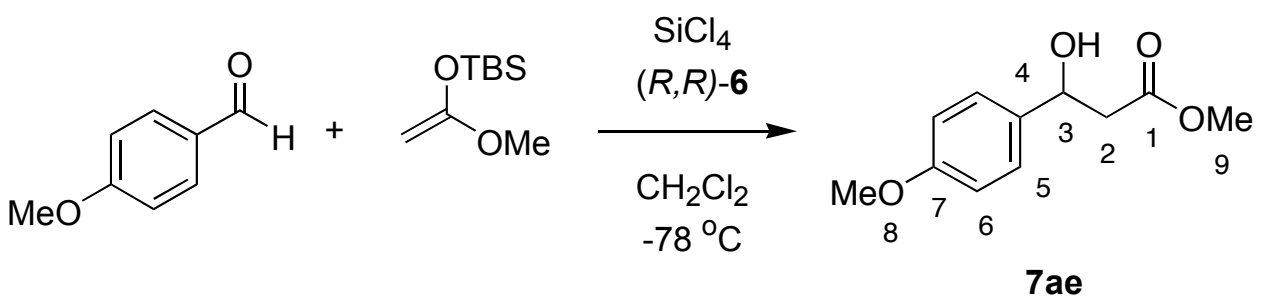

Following General Procedure 3, $243 \mu \mathrm{L}(2.0 \mathrm{mmol})$ of anisaldehyde was combined with $84 \mathrm{mg}$ (0.1 mmol, 0.05 equiv) of bis-phosphoramide $((R, R)-6), 252 \mu \mathrm{L}\left(2.2 \mathrm{mmol}, 1.1\right.$ equiv) of $\mathrm{SiCl}_{4}$, and $523 \mu \mathrm{L}$ (2.4 mmol, 1.2 equiv) of 1-(tert-butyldimethylsilyloxy)-1-methoxyethene (1a) to yield, after purification by silica gel column chromatography (hexanes/EtOAc, 2/1), $410 \mathrm{mg}$ (97\%) of methyl 3-hydroxy-3-(4-methoxyphenyl) propanoate (7ae) as a white solid. After recrystallization from benzene/hexanes, analytically pure, colorless needles were isolated.

Data for 7ae:

mp: $\quad 67-68^{\circ} \mathrm{C}$ (benzene/hexanes)

1 NMR: $\quad\left(400 \mathrm{MHz}, \mathrm{CDCl}_{3}\right)$

7.30 (m, 2 H, HC(5)), 6.89 (m, 2 H, HC(6)), 5.09 (dt, J=9.0, 3.7, 1 H, HC(3)),

3.80 (s, 3 H, HC(8)), 3.72 (s, 3 H, HC(9)), 3.10 (d, $J=3.4,1 \mathrm{H}, \mathrm{OH}), 2.73$ (dd, $J$

$=16.4,8.7,1 \mathrm{H}, \mathrm{H} H \mathrm{C}(2)), 2.72(\mathrm{dd}, J=16.4,6.6,1 \mathrm{H}, H \mathrm{HC}(2))$

${ }^{13} \mathrm{C} \mathrm{NMR}: \quad\left(100 \mathrm{MHz}, \mathrm{CDCl}_{3}\right)$

172.74 (C(1)), $159.11(\mathrm{C}(7)), 134.50$ (C(4)), 126.84 (C(5)), 113.81 (C(6)), 69.84

(C(3)), 55.18 (C(8)), $51.79(\mathrm{C}(9)), 43.00(\mathrm{C}(2))$

IR: (neat)

3439 (s), 3001 (w), 2953 (m), 2837 (m), 1735 (s), 1612 (m), 1586 (w), 1514 (s), 1438 (m), 1358 (w), 1302 (m), 1248 (s), 1201 (m), 1174 (m), $1111(\mathrm{w}), 1032$ (s), $833(\mathrm{~m})$.

MS: $\quad(E I, 70 \mathrm{eV})$

$210\left(\mathrm{M}^{+}, 10\right), 161$ (10), 138 (10), 137 (100) 
TLC: $\quad R_{f} 0.20$ (hexanes/EtOAc, 2/1) [UV(254)/PMA]

Opt. Rot.: $\quad[\alpha]_{\mathrm{D}}^{24}+14.4(\mathrm{c}=2.05$, EtOH $)$

SFC: $\quad t_{R}(R)-7 \mathbf{a e}, 3.95$ (98.7\%); $t_{R}(S)-7 \mathbf{a e}, 4.45 \min (1.3 \%)$ (Chiralpak AD, 150 psi, 40

${ }^{\circ} \mathrm{C}, 6.0 \% \mathrm{CH}_{3} \mathrm{OH}$ in $\mathrm{CO}_{2}, 3.0 \mathrm{~mL} / \mathrm{min}, 220 \mathrm{~nm}$ )

Analysis: $\quad \mathrm{C}_{11} \mathrm{H}_{14} \mathrm{O}_{4}(210.23)$

Calcd: $\quad$ C, $62.85 ; \quad \mathrm{H}, 6.71 \%$

Found: $\quad \mathrm{C}, 62.83 ; \quad \mathrm{H}, 6.75 \%$

Preparation of Methyl 3-Hydroxy-3-(4-trifluoromethylphenyl)propanoate (7af)<smiles>C=C(O[GaH2])OC(=O)C[CH+]C(=O)c1ccc(C(F)(F)F)cc1</smiles>

Following General Procedure 3, $273 \mu \mathrm{L}(2.0 \mathrm{mmol})$ of 4-trifluoromethylbenzaldehyde was combined with $84 \mathrm{mg}(0.1 \mathrm{mmol}, 0.05$ equiv) of bis-phosphoramide $((R, R)-6), 252 \mu \mathrm{L}(2.2$ mmol, 1.1 equiv) of $\mathrm{SiCl}_{4}$, and $523 \mu \mathrm{L}$ (2.4 mmol, 1.2 equiv) of 1-(tert-butyldimethylsilyloxy)-1methoxyethene (1a) to yield, after purification by silica gel column chromatography (hexanes/EtOAc, 2/1), $484 \mathrm{mg}$ (97\%) of methyl-3-hydroxy-3-(4-trifluoromethylphenyl) propanoate (7af) as a white solid. Recrystallization from hexane afforded analytically pure, colorless needles.

Data for 7af:

mp: $\quad 42-43^{\circ} \mathrm{C}$ (hexane)

${ }^{1} \mathrm{H}$ NMR: $\quad\left(400 \mathrm{MHz}, \mathrm{CDCl}_{3}\right)$

7.58 (d, $J=8.3,2 \mathrm{H}, \mathrm{HC}(5)), 7.46$ (d, $J=8.0,2 \mathrm{H}, \mathrm{HC}(6)), 5.15$ (m, $1 \mathrm{H}, \mathrm{HC}(3)$ ),

3.75 (d, $J=3.7,1 \mathrm{H}, \mathrm{OH}), 3.68$ (s, $3 \mathrm{H}, \mathrm{HC}(9)), 2.71$ (m, $2 \mathrm{H}, \mathrm{HC}(2))$

${ }^{13} \mathrm{C}$ NMR: $\quad\left(100 \mathrm{MHz}, \mathrm{CDCl}_{3}\right)$

$172.72(\mathrm{C}(1)), 146.68(\mathrm{C}(4)), 130.10$ (q, $J=32.0 \mathrm{C}(7)), 126.13(\mathrm{C}(5)), 125.60$

(C(6)), 124.22 (q, $J=271.8 \mathrm{C}(8)), 69.78$ (C(3)), 52.14 (C(9)), 43.15 (C(2))

${ }^{19}$ F NMR: $\quad\left(376 \mathrm{MHz}, \mathrm{CDCl}_{3}\right)$

$-63.1\left(\mathrm{~F}_{3} \mathrm{C}(8)\right)$ 
IR: (neat)

3456 (s), 3005 (w), 2957 (m), 2851 (w), 1734 (s), 1621 (m), 1439 (m), 1416 (m), 1327 (s), 1272 (w), 1164 (s), 1124 (s), 1068 (s), 1017 (m), 845 (m)

MS: $\quad(\mathrm{CI})$

$248\left(\mathrm{M}^{+}, 8\right), 230$ (43), 223 (21), 212 (10), 184 (11), 175 (27), 168 (26), 157 (100)

TLC: $\quad R_{f} 0.25$ (hexanes/EtOAc, 2/1) [UV(254)/PMA]

Opt. Rot.: $\quad[\alpha]_{\mathrm{D}}^{24}+13.60(\mathrm{c}=2.27, \mathrm{EtOH})$

SFC: $\quad t_{R}(R)-7$ af, $3.75(95.7 \%) ; t_{R}(S)$-7af, $4.14 \min (4.3 \%)$ (Chiralpak AS, 150 psi, 40 ${ }^{\circ} \mathrm{C}, 7.5 \% \mathrm{CH}_{3} \mathrm{OH}$ in $\mathrm{CO}_{2}, 2.5 \mathrm{~mL} / \mathrm{min}, 220 \mathrm{~nm}$ )

Analysis: $\quad \mathrm{C}_{11} \mathrm{H}_{11} \mathrm{~F}_{3} \mathrm{O}_{3}(248.20)$

Calcd: $\quad$ C, $53.23 ; \quad \mathrm{H}, 4.47 \%$

Found: $\quad$ C, $53.49 ; \quad H, 4.61 \%$

Preparation of Methyl 3-Hydroxy-5-phenyl-4-pentenoate (7ag)

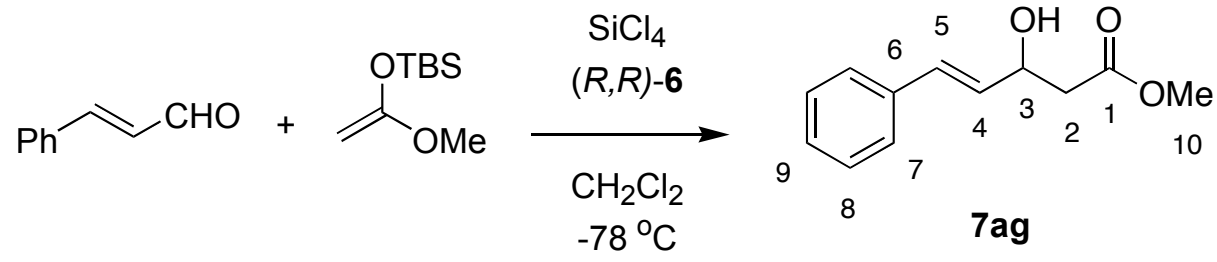

Following General Procedure 3, $252 \mu \mathrm{L}(2.0 \mathrm{mmol})$ of cinnamaldehyde was combined with 84 $\mathrm{mg}(0.1 \mathrm{mmol}, 0.05$ equiv) of bis-phosphoramide $((R, R)-6), 252 \mu \mathrm{L}$ (2.2 mmol, 1.1 equiv) of $\mathrm{SiCl}_{4}$, and $523 \mu \mathrm{L}$ (2.4 mmol, 1.2 equiv) of 1-(tert-butyldimethylsilyloxy)-1-methoxyethene (1a) to yield, after purification by silica gel column chromatography (hexanes/EtOAc, 2/1), $393 \mathrm{mg}$ (95\%) of 3-hydroxy-5-phenyl-4-pentenoate (7ag) as a colorless oil. NMR spectral data matched that previously reported in the literature. ${ }^{13}$

\section{Data for 7ag:}

${ }^{1} \mathrm{H}$ NMR: $\quad\left(400 \mathrm{MHz}, \mathrm{CDCl}_{3}\right)$

7.39 (bd, $J=7.1,2 \mathrm{H}, 2 \times \mathrm{HC}(7)), 7.34$ (bt, $J=7.12 \mathrm{H}, 2 \times \mathrm{HC}(8)), 7.28$ (bt, $J=$ 6.1, $1 \mathrm{H}, \mathrm{HC}(9)), 6.67$ (d, $J=15.9,1 \mathrm{H}, \mathrm{HC}(5)), 6.24(\mathrm{dd}, J=15.9,6.1,1 \mathrm{H}$, HC(4)), 4.75 (m, 1 H, HC(3)), 3.74 (s, 3 H, H(C(10)), 3.23 (d, J=4.6, 1 H, HO), 2.67 (dd, $J=16.4,1.2,1 \mathrm{H}, \mathrm{H} H \mathrm{C}(2)), 2.66$ (dd, $J=16.4,3.0,1 \mathrm{H}, H \mathrm{HC}(2))$ 
${ }^{13} \mathrm{C}$ NMR: $\quad\left(100 \mathrm{MHz}, \mathrm{CDCl}_{3}\right)$

$172.74(\mathrm{C}(1)), 136.50$ (C(6)), 130.89 (C(5)), $130.06(\mathrm{C}(4)), 128.70$ (C(7)), 127.95

$(\mathrm{C}(9)), 126.67(\mathrm{C}(8)), 68.98(\mathrm{C}(3)), 52.03(\mathrm{C}(10)), 41.50(\mathrm{C}(2))$

IR: (neat)

3448 (s), 3058 (w), 3026 (m), 2952 (m), 1735 (s), 1494 (m), 1438 (s), 1403 (w), 1357 (m), 1283 (m), 1205 (m), 1166 (s), 1104 (m), 1030 (m), 969 (s), 751 (s), 694

(s)

Opt. Rot.: $\quad[\alpha]_{\mathrm{D}}^{24}-17.0(\mathrm{c}=2.07, \mathrm{EtOH})$

SFC: $\quad t_{R}(R)-7 \mathbf{a g}, 3.61$ (96.9\%); $t_{R}(S)-7 \mathbf{a g}, 4.24 \mathrm{~min}(3.1 \%)$ (Chiralpak OD, $150 \mathrm{psi}, 40$ ${ }^{\circ} \mathrm{C}, 7.5 \% \mathrm{CH}_{3} \mathrm{OH}$ in $\mathrm{CO}_{2}, 3.0 \mathrm{~mL} / \mathrm{min}, 220 \mathrm{~nm}$ )

\section{Preparation of Methyl 3-Hydroxy-4-methyl-5-phenyl-4-pentenoate (7ah)}

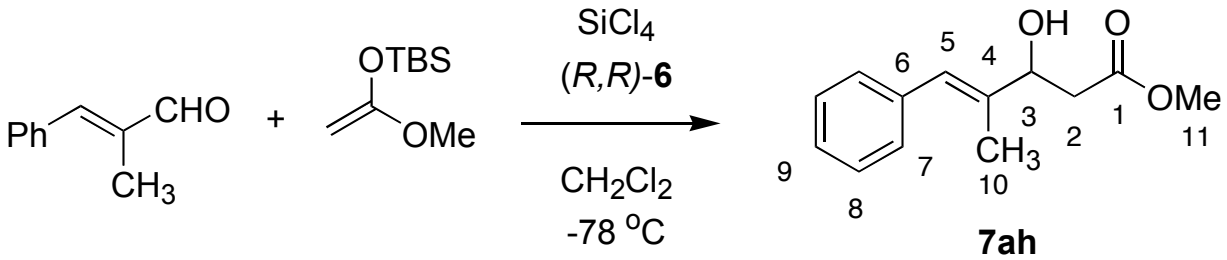

Following General Procedure 3, $279 \mu \mathrm{L}(2.0 \mathrm{mmol})$ of $\alpha$-methylcinnamaldehyde was combined with $84 \mathrm{mg}$ ( $0.1 \mathrm{mmol}, 0.05$ equiv) of bis-phosphoramide ((R,R)-6), $252 \mu \mathrm{L}(2.2 \mathrm{mmol}, 1.1$ equiv) of $\mathrm{SiCl}_{4}$, and $523 \mu \mathrm{L}(2.4 \mathrm{mmol}, 1.2$ equiv) of 1-(tert-butyldimethylsilyloxy)-1methoxyethene (1a) to yield, after purification by silica gel column chromatography (hexanes/EtOAc, 2/1), $432 \mathrm{mg}$ (98\%) of methyl 3-hydroxy-4-methyl-5-phenyl-4-pentenoate (7ah) as a colorless oil. Kugelrohr distillation afforded an analytically pure, colorless oil. NMR spectral data matched that previously reported in the literature. ${ }^{14}$

Data for 7ah:

mp: $\quad 115^{\circ} \mathrm{C}\left(1.3 \times 10^{-4} \mathrm{mmHg}, \mathrm{ABT}\right)$

${ }^{1} \mathrm{H}$ NMR: $\quad\left(400 \mathrm{MHz}, \mathrm{CDCl}_{3}\right)$

7.35 (m, 2 H, HC(7)), 7.25 (m, 3 H, HC(8), HC(9)), 6.62 (bs, 1 H, HC(5)), 4.64

(m, $1 \mathrm{H}, \mathrm{HC}(3)), 3.76$ (s, $\left.3 \mathrm{H}, \mathrm{H}_{3} \mathrm{C}(11)\right), 2.94$ (d, J=3.4, $\left.1 \mathrm{H}, \mathrm{HO}\right), 2.68$ (m, $2 \mathrm{H}$, 2 x $\mathrm{HC}(2)), 1.90\left(\mathrm{~d}, J=1.5,3 \mathrm{H}, \mathrm{H}_{3} \mathrm{C}(10)\right)$

${ }^{13} \mathrm{C} \mathrm{NMR}: \quad\left(100 \mathrm{MHz}, \mathrm{CDCl}_{3}\right)$ 
173.23 (C(1)), 138.26 (C(4)), 137.45 (C(6)), 129.20 (C(8)), 128.35 (C(9)), 126.83 (C(7)), 126.26 (C(6)), 73.77 (C(3)), 52.18 (C(11)), 40.28 (C(2)), 14.03 (C(10))

IR: (neat)

3456 (s), 3024 (w), 2984 (w), 2952 (m), 2917 (w), 1736 (s), 1599 (m), 1492 (m), 1439 (s), 1347 (m), 1273 (s), 1169 (s), 1074 (m), 1042 (m), 990 (m), 920 (w), 870 (w), $753(\mathrm{~m})$

$\underline{\mathrm{MS}}: \quad(\mathrm{CI})$

$220\left(\mathrm{M}^{+}, 29\right), 202$ (17), 147 (100), 129 (74), 118 (63)

TLC: $\quad R_{f} 0.31$ (hexanes/EtOAc, 2/1) [UV(254)/PMA]

Opt. Rot.: $\quad[\alpha]_{\mathrm{D}}^{24}-13.70(\mathrm{c}=2.23, \mathrm{EtOH})$

SFC: $\quad t_{R}(R)-7 \mathbf{a h}, 4.34(72.7 \%) ; t_{R}(S)-7 \mathbf{a h}, 4.45 \min (27.3 \%)$ (Chiralpak AD, 150 psi, $40{ }^{\circ} \mathrm{C}, 6.0 \% \mathrm{CH}_{3} \mathrm{OH}$ in $\mathrm{CO}_{2}, 3.0 \mathrm{~mL} / \mathrm{min}, 220 \mathrm{~nm}$ )

Analysis: $\quad \mathrm{C}_{13} \mathrm{H}_{16} \mathrm{O}_{3}(220.26)$

Calcd: $\quad$ C, $70.89 ; \quad \mathrm{H}, 7.32 \%$

Found: $\quad$ C, $70.61 ; \quad$ H, $7.33 \%$

\section{Preparation of Methyl 3-Hydroxy-3-(2-furyl)propanoate (7ai)}

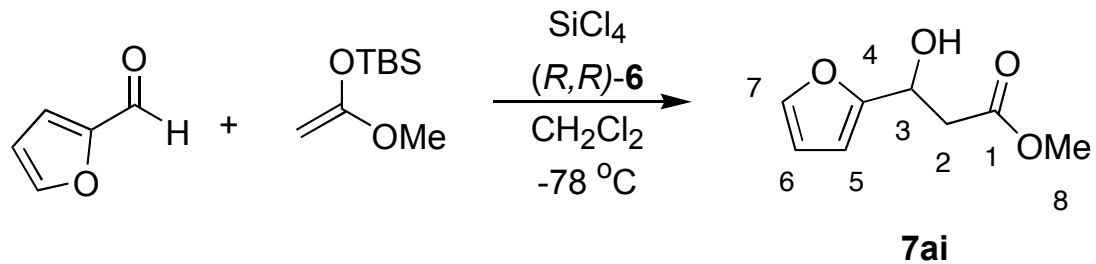

Following General Procedure 3, $165 \mu \mathrm{L}(2.0 \mathrm{mmol})$ of 2-furfuraldehyde was combined with 84 $\mathrm{mg}(0.1 \mathrm{mmol}, 0.05$ equiv) of bis-phosphoramide $((R, R)-6), 252 \mu \mathrm{L}$ (2.2 mmol, 1.1 equiv) of $\mathrm{SiCl}_{4}$, and $523 \mu \mathrm{L}$ (2.4 mmol, 1.2 equiv) of 1-(tert-butyldimethylsilyloxy)-1-methoxyethene (1a) to yield, after purification by silica gel column chromatography (hexanes/EtOAc, 2/1), $320 \mathrm{mg}$ (94\%) of methyl 3-hydroxy-3-(2-furyl) propanoate (7ai) as a colorless oil. 


\section{Data for 7ai:}

bp: $\quad 75^{\circ} \mathrm{C}\left(2.2 \times 10^{-4} \mathrm{mmHg}, \mathrm{ABT}\right)$

11 $\mathrm{H}$ NMR: $\quad\left(400 \mathrm{MHz}, \mathrm{CDCl}_{3}\right)$

7.38 (m, $1 \mathrm{H}, \mathrm{HC}(7)), 6.33$ (m, $1 \mathrm{H}, \mathrm{HC}(6)), 6.27$ (m, $1 \mathrm{H}, \mathrm{HC}(5)), 5.12$ (d, $J=$ 4.4, 1 H, HC(3)), 3.73 (s, 3 H, HC(8)), 3.21 (bs, 1 H, HO), 2.90 (dd, J= 16.4, 6.3, $1 \mathrm{H}, \mathrm{H} H \mathrm{C}(2)), 2.88(\mathrm{dd}, J=16.4,5.4,1 \mathrm{H}, H \mathrm{HC}(2))$

${ }^{13} \mathrm{C} \mathrm{NMR}: \quad\left(100 \mathrm{MHz}, \mathrm{CDCl}_{3}\right)$

$172.64(\mathrm{C}(1)), 154.83(\mathrm{C}(4), 142.56(\mathrm{C}(7)), 110.52$ (C(6)), $106.62(\mathrm{C}(5)), 64.38$ (C(3)), $52.28(\mathrm{C}(8)), 39.78(\mathrm{C}(2))$

IR: (neat)

3460 (s), 2955 (m), 1736 (s), 1504 (w), 1439 (m), 1363 (m), 1284 (m), 1211 (m), 1164 (m), 1066 (w), 1030 (m), 1011 (m), 741 (m)

MS: $\quad(\mathrm{EI}, 70 \mathrm{eV})$

$170\left(\mathrm{M}^{+}, 18\right), 110(14), 97$ (100), 84 (46), 71 (13)

TLC: $\quad R_{f} 0.30$ (hexanes/EtOAc, 2/1) [UV(254)/PMA]

Opt. Rot.: $\quad[\alpha]_{\mathrm{D}}^{24}+22.65(\mathrm{c}=2.15, \mathrm{EtOH})$

SFC: $\quad t_{R}(R)-7 \mathbf{a i}, 4.02(93.4 \%) ; t_{R}(S)$-7ai, $4.55 \mathrm{~min}(6.6 \%)$ (Chiralpak AS, 150 psi, 40 ${ }^{\circ} \mathrm{C}, 7.5 \% \mathrm{CH}_{3} \mathrm{OH}$ in $\mathrm{CO}_{2}, 2.5 \mathrm{~mL} / \mathrm{min}, 220 \mathrm{~nm}$ )

Preparation of Methyl 3-Hydroxy-3-cyclohexylpropanoate (7aj)

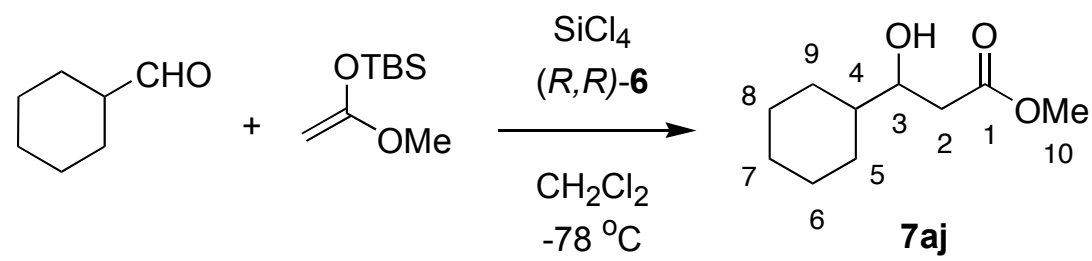

A flame-dried, 20-mL, 2-neck flask containing a solution of $168 \mathrm{mg}(0.2 \mathrm{mmol}, 0.1$ equiv) of bis-phosphoramide $((R, R)-6)$ in $\mathrm{CH}_{2} \mathrm{Cl}_{2}(10 \mathrm{~mL})$ was cooled to $-78{ }^{\circ} \mathrm{C}$ under nitrogen in a dry ice/isopropanol bath. Into this reaction mixture was added $242 \mu \mathrm{L}(2.0 \mathrm{mmol})$ of cyclohexanecarboxaldehyde. To the resulting mixture $252 \mu \mathrm{L}(2.2 \mathrm{mmol}, 1.1$ equiv $)$ of $\mathrm{SiCl}_{4}$ was added and the reaction mixture was allowed to stir at $-78{ }^{\circ} \mathrm{C}$ for $5 \mathrm{~min}$ in a dry ice/isopropanol bath. Then $523 \mu \mathrm{L}$ (2.4 mmol, 1.2 equiv) of 1-(tert-butyldimethylsilyloxy)-1- 
methoxyethene (1a) was added dropwise (over $5 \mathrm{~min}$ ) the resulting mixture was allowed to stir at $-78{ }^{\circ} \mathrm{C}$ for $6 \mathrm{~h}$ in a dry ice/isopropanol bath. The cold reaction mixture was then poured into a rapidly stirring $1 / 1$ sat. aq. $\mathrm{KF} / 1.0 \mathrm{M} \mathrm{KH}_{2} \mathrm{PO}_{4}$ solution $(50 \mathrm{~mL})$. This biphasic mixture was stirred vigorously for $2 \mathrm{~h}$ after which the aqueous layer was washed with $\mathrm{CH}_{2} \mathrm{Cl}_{2}(3 \mathrm{x} 50 \mathrm{~mL})$. The combined organic extracts were dried over $\mathrm{Na}_{2} \mathrm{SO}_{4}$, filtered and the filtrate was concentrated in vacuo. The residue was purified by silica gel column chromatography (hexanes/EtOAc, 2/1). The product-containing fractions were combined and the solvent was removed in vacuo to yield $321 \mathrm{mg}(86 \%)$ of methyl 3-hydroxy-3-cyclohexylpropanoate (7aj) as a colorless oil. NMR spectral data matched that previously reported in the literature. Analysis of the enantiomeric composition was preformed on the trifluoroacetate derivative, $\mathbf{3 1}$.

\section{Data for 7aj:}

${ }^{1} \mathrm{H}$ NMR: $\quad\left(400 \mathrm{MHz}, \mathrm{CDCl}_{3}\right)$

3.78 (m, $1 \mathrm{H}, \mathrm{HC}(3)), 3.71$ (s, $3 \mathrm{H}, \mathrm{HC}(10)), 2.81$ (d, J = 4.1, $1 \mathrm{H}, \mathrm{HO}), 2.52$ (dd, $J=16.1,2.7,1 \mathrm{H}, \mathrm{H} H \mathrm{C}(2)), 2.43(\mathrm{dd}, J=16.1,9.4,1 \mathrm{H}, H \mathrm{HC}(2)), 1.86$ (bd, $1 \mathrm{H}$, $\mathrm{C}(9))$, 1.80-1.72 (m, 2 H, 2 x HC(8), 1.66 (m, 2 H, 2 x HC(5)) 1.42-1.32 (m, 1 H, $\mathrm{HC}(4)), 1.30$ - 0.95 (m, 5 H, 2 x HC(7), HC(9), 2 x HC(6))

${ }^{13} \mathrm{C} \mathrm{NMR}: \quad\left(100 \mathrm{MHz}, \mathrm{CDCl}_{3}\right)$

174.20 (C(1)), 72.35 (C(3)), 52.10 (C(10)), 43.23 (C11)), 38.56 (C(2)), 28.98 (C(7)), 28.43 (C(5)), 26.60 (C(6)), $26.34(\mathrm{C}(8)), 26.23$ (C(9))

IR: (neat)

3462 (s), 2926 (s), 2853 (s), 1737 (s), 1439 (s), 1407 (w), 1366 (w), 1311 (w), $1281(\mathrm{w}), 1192(\mathrm{w}), 1168(\mathrm{~m}), 992(\mathrm{~m}), 892(\mathrm{~m}), 843(\mathrm{w})$

GC: $\quad t_{R}(S)-31,7.73(5.8 \%) ; t_{R}(R)-31,8.20 \min (94.2 \%)\left(\mathrm{GTA}, 16 \mathrm{psi}, 100{ }^{\circ} \mathrm{C}\right.$ isothermal)

Opt. Rot.: $[\alpha]_{\mathrm{D}}^{24}+17.5(\mathrm{c}=2.84, \mathrm{EtOH})$ 


\section{Preparation of Methyl 3-Trifluoromethylacetoxy-3-cyclohexylpropanoate ((3R)-31)}

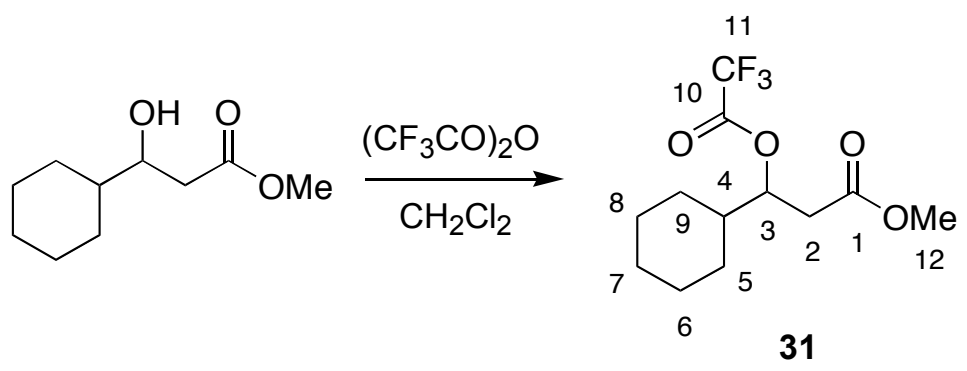

To a stirred solution of $61 \mathrm{mg}(1.1 \mathrm{mmol})$ of $(3 R)-7 \mathbf{a j}$ in $\mathrm{CH}_{2} \mathrm{Cl}_{2}(1.0 \mathrm{~mL})$ was added $0.25 \mathrm{~mL}$ (1.76 mmol, 1.6 equiv) of trifluoroacetic anhydride at room temperature under nitrogen. After the addition was completed, the mixture was stirred for $12 \mathrm{~h}$. The reaction mixture was then concentrated in vacuo. Purification of the residue by silica gel column chromatography (hexane/EtOAc, 10/1), afforded $88 \mathrm{mg}$ (95\%) of methyl 3-trifluoromethylacetoxy-3cyclohexylpropanoate $((3 R)-\mathbf{3 1})$.

Data for $(3 R)-\mathbf{3 1}$ :

${ }^{1} \mathrm{H}$ NMR: $\quad\left(400 \mathrm{MHz}, \mathrm{CDCl}_{3}\right)$

$5.33(\mathrm{dd}, J=6.6,6.1,1 \mathrm{H}, \mathrm{HC}(3)), 3.69$ (s, $3 \mathrm{H}, \mathrm{HC}(12)), 2.69$ (d, $J=6.8,2 \mathrm{H}, 2$

x HC(2)), 1.81-1.64 (m, 6 H, H(Cy)), 1.28-1.00 (m, 5 H, H(Cy))

${ }^{13} \mathrm{C} \mathrm{NMR:} \quad\left(100 \mathrm{MHz}, \mathrm{CDCl}_{3}\right)$

170.56 (C(1)), 157.10 (q, $J=41.9, \mathrm{C}(10)), 114.80$ (q, $J=286, \mathrm{C}(11)), 78.83$ (C(3)), 52.23 (C(12)), $41.27(\mathrm{C}(2)), 36.18$ (C(4)), $28.66(\mathrm{C}(7)), 27.87(\mathrm{C}(5))$,

$26.17(\mathrm{C}(9)), 25.93(\mathrm{C}(6)), 25.85(\mathrm{C}(8))$

IR: (neat)

2934 (s), 2858 (s), 1787 (s), 1745 (s), 1451 (m), 1439 (m), 1383 (m), 1339 (m), 1285 (m), 1262 (s), 1220 (s), 992 (m), 892 (w), 837 (w), 771 (m), 732 (m)

GC: $\quad t_{R}(3 S)-31,7.73(5.8 \%) ; t_{R}(3 R)-31,8.20 \min (94.2 \%)\left(\mathrm{GTA}, 16 \mathrm{psi}, 100{ }^{\circ} \mathrm{C}\right.$ isothermal) 


\section{Preparation of Methyl 3-Hydroxy-5-phenylpentanoate (7ak)}

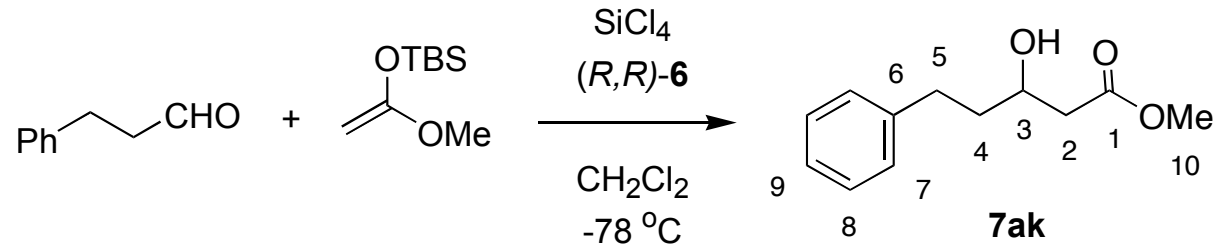

A flame-dried, 20-mL, 2-neck flask containing a solution of $168 \mathrm{mg}$ ( $0.2 \mathrm{mmol}, 0.1$ equiv) of bis-phosphoramide $((R, R)-6)$ in $\mathrm{CH}_{2} \mathrm{Cl}_{2}(10 \mathrm{~mL})$ was cooled to $-78{ }^{\circ} \mathrm{C}$ under nitrogen in a dry ice/isopropanol bath. To this reaction mixture was added $263 \mu \mathrm{L}(2.0 \mathrm{mmol})$ of hydrocinnamaldehyde. To the resulting mixture was added $252 \mu \mathrm{L}$ (2.2 mmol, 1.1 equiv) of $\mathrm{SiCl}_{4}$ and the reaction mixture was allowed to stir at $-78{ }^{\circ} \mathrm{C}$ for $5 \mathrm{~min}$ in a dry ice/isopropanol bath. Then $523 \mu \mathrm{L}(2.4 \mathrm{mmol}, 1.2$ equiv) of 1-(tert-butyldimethylsilyloxy)-1-methoxyethene (1a) was added dropwise (over $5 \mathrm{~min}$ ) to the reaction mixture. The resulting mixture was allowed to stir at $-78^{\circ} \mathrm{C}$ for $6 \mathrm{~h}$ in a dry ice/isopropanol bath. The cold reaction mixture was then poured into a rapidly stirring $1 / 1$ sat. aq. $\mathrm{KF} / 1.0 \mathrm{M} \mathrm{KH}_{2} \mathrm{PO}_{4}$ solution $(50 \mathrm{~mL})$. This biphasic mixture was stirred vigorously for $2 \mathrm{~h}$ after which the aqueous layer was washed with $\mathrm{CH}_{2} \mathrm{Cl}_{2}(3 \times 50$ $\mathrm{mL}$ ). The combined organic extracts were dried over $\mathrm{Na}_{2} \mathrm{SO}_{4}$, filtered and the filtrate was concentrated in vacuo. The residue was purified by silica gel column chromatography (hexanes/EtOAc, 2/1). The product-containing fractions were combined and the solvent was removed in vacuo to yield $300 \mathrm{mg}$ (72\%) of methyl 3-hydroxy-5-phenylpentanoate (7ak) as a colorless oil. NMR spectral data matched that previously reported in the literature. ${ }^{15}$

Data for 7ak:

${ }^{1} \mathrm{H}$ NMR: $\quad\left(400 \mathrm{MHz}, \mathrm{CDCl}_{3}\right)$

7.28-7.18 (m, 5H, HC(7,8,9)), 4.01 (m, $1 \mathrm{H}, \mathrm{HC}(3)), 3.70$ (s, $\left.3 \mathrm{H}, \mathrm{H}_{3} \mathrm{C}(10)\right), 3.10$ (d, $J=3.6,1 \mathrm{H}, \mathrm{OH}), 2.82(\mathrm{~m}, 1 \mathrm{H}, \mathrm{H} H \mathrm{C}(5)), 2.69$ (m, $1 \mathrm{H}, \mathrm{H} H \mathrm{C}(5)), 2.50$ (dd, $J$ $=16.5,3.4,1 \mathrm{H}, \mathrm{H} H \mathrm{C}(2)), 2.46(\mathrm{dd}, J=16.5,8.7,1 \mathrm{H}, \mathrm{H} H \mathrm{C}(2)), 1.84(\mathrm{~m}, 1 \mathrm{H}$, $\mathrm{H} H \mathrm{C}(4)), 1.74$ (m, $1 \mathrm{H}, \mathrm{H} H \mathrm{C}(4))$,

${ }^{13} \mathrm{C} \mathrm{NMR:} \quad\left(100 \mathrm{MHz}, \mathrm{CDCl}_{3}\right)$

173.31 (C(1)), $141.61(\mathrm{C}(6)), 128.38$ (C(7)), 128.35 (C(8)), 125.83 (C(9)), 67.12 $(\mathrm{C}(3)), 51.72(\mathrm{C}(10)), 41.07(\mathrm{C}(5)), 38.05(\mathrm{C}(2)), 31.69$ (C(4))

IR: (neat)

3450 (w), 2951 (s), 2929 (s), 2862 (w), 1737 (s), 1603 (w), 1496 (m), 1454 (s), 
1439 (s), 1409 (m), 1359 (m), 1303 (s), 1254 (s), 1198 (s), 1177 (s), 1158 (s), $1082(\mathrm{~s}), 1060(\mathrm{~s}), 1045(\mathrm{~s}), 1031(\mathrm{~m}), 1002(\mathrm{~m})$

SFC: $\quad t_{R}(S)$-7ak, $3.46(90.7 \%) ; t_{R}(R)-7 \mathbf{a k}, 4.13 \mathrm{~min}$ (9.3\%) (Chiralpak OD, $150 \mathrm{psi}, 40$ ${ }^{\circ} \mathrm{C}, 5.0 \% \mathrm{CH}_{3} \mathrm{OH}$ in $\mathrm{CO}_{2}, 3.0 \mathrm{~mL} / \mathrm{min}, 220 \mathrm{~nm}$ )

Opt. Rot.: $\quad[\alpha]_{D}^{24}+17.5(\mathrm{c}=2.84, \mathrm{EtOH})$

General Procedure 4. Preparation of tert-butyl 3-Hydroxy-3-phenylpropanoate (7ba)

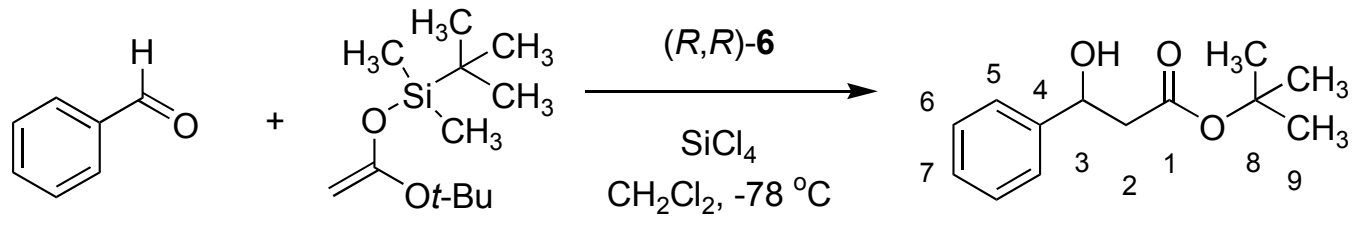

$7 \mathrm{ba}$

A flame-dried, $25 \mathrm{~mL}, 2$-neck flask containing a solution of $17 \mathrm{mg}(0.02 \mathrm{mmol}, 0.01$ equiv) of bis-phosphoramide $((R, R)-6)$ in $\mathrm{CH}_{2} \mathrm{Cl}_{2}(10 \mathrm{~mL})$ was cooled to $-78{ }^{\circ} \mathrm{C}$ and then $200 \mu \mathrm{L}(2.0$ mmol) of benzaldehyde was added in one portion. To the resulting solution, $250 \mu \mathrm{L}(2.2 \mathrm{mmol}$, 1.1 equiv) of $\mathrm{SiCl}_{4}$ was added and the reaction mixture was allowed to stir at $-78{ }^{\circ} \mathrm{C}$ for $5 \mathrm{~min}$. Then, $552 \mathrm{mg}$ ( $2.4 \mathrm{mmol}, 1.2$ equiv) of $\mathbf{1 b}$ was added dropwise (over $1 \mathrm{~min}$ ) to the reaction mixture. The resulting mixture was allowed to stir at $-78{ }^{\circ} \mathrm{C}$ for $1 \mathrm{~h}$ whereupon the cold reaction mixture was poured into a rapidly stirring solution of saturated aqueous $\mathrm{NaHCO}_{3}(50 \mathrm{~mL})$ and the resulting mixture was stirred for $3 \mathrm{~h}$ at room temperature. The solution was then filtered through Celite and the filter cake was washed with $\mathrm{CH}_{2} \mathrm{Cl}_{2}(100 \mathrm{~mL})$. The aqueous layer was washed with $\mathrm{CH}_{2} \mathrm{Cl}_{2}(50 \mathrm{~mL})$ and the combined organics were dried over $\mathrm{K}_{2} \mathrm{CO}_{3}(5 \mathrm{~g})$ and concentrated in vacuo to give a colorless oil $(480 \mathrm{mg})$. Crude ${ }^{1} \mathrm{H}$ NMR analysis indicated that a 4.4:1 mixture of the tert-butyl ester $\mathbf{7} \mathbf{b a}$ and the tert-butyldimethylsilyl ester $\mathbf{8 a}$ had formed. The residue was dissolved in $\mathrm{CH}_{2} \mathrm{Cl}_{2}(50 \mathrm{~mL})$ poured into a stirred solution of 1/1 sat. aq. $\mathrm{KF} / 1.0 \mathrm{M}$ $\mathrm{KH}_{2} \mathrm{PO}_{4}(50 \mathrm{~mL})$ and the resulting mixture was stirred for $3 \mathrm{~h}$ after which it was diluted with water $(100 \mathrm{~mL})$ and the aqueous layer washed with $\mathrm{CH}_{2} \mathrm{Cl}_{2}(3 \times 50 \mathrm{~mL})$. The combined organic extracts were dried over $\mathrm{K}_{2} \mathrm{CO}_{3}(5 \mathrm{~g})$, filtered through Celite and concentrated in vacuo. The residue was purified by Kugelrohr distillation to yield $302 \mathrm{mg}(68 \%)$ of $7 \mathbf{b a}$ as a colorless oil. The NMR spectral data matched that previously reported in the literature. ${ }^{16,17}$ 


\section{Data for 7ba:}

bp: $\quad 200{ }^{\circ} \mathrm{C}(0.5 \mathrm{mmHg}, \mathrm{ABT})$

${ }^{1} \mathrm{H}$ NMR: $\quad\left(500 \mathrm{MHz}, \mathrm{CDCl}_{3}\right)$

7.40-7.32 (m, 4 H, HC(5,6)), 7.30-7.26 (m, 1 H, HC(7)), 5.09 (dd, J=8.5, 4.0, 1 H, HC(3)), 3.48 (br s, 1 H, OH), 2.68 (dd, $J=17.0,8.5,1$ H, HHC(2)), 2.64 (dd, $J$ $=17.0,4.0,1 \mathrm{H}, \mathrm{H} H \mathrm{C}(2)), 1.45(\mathrm{~s}, 9 \mathrm{H}, \mathrm{HC}(9))$

${ }^{13} \mathrm{C} \mathrm{NMR}: \quad\left(125 \mathrm{MHz}, \mathrm{CDCl}_{3}\right)$

172.18 (C(1)), 142.83 (C(4)), 128.71 (C(5)), 127.90 (C(7)), 125.95 (C(6)), 81.75 (C(8)), 70.61 (C(3)), 44.45 (C(2)), 28.27 (C(9))

IR: (thin film)

$3435(\mathrm{~m}), 2977(\mathrm{~m}), 1718$ (s)

MS: (ESI)

$245\left(\mathrm{M}^{+\mathrm{Na}}, 100\right), 229(32)$

HRMS: $\quad$ calcd for $\mathrm{C}_{13} \mathrm{H}_{18} \mathrm{O}_{3} \mathrm{Na}: 245.1154$, found 245.1152

TLC: $\quad R_{f} 0.3$ (hexanes/EtOAc, 4/1) [UV(254)/KMnO $\left.{ }_{4}\right]$

Opt. Rot.: $\quad[\alpha]_{\mathrm{D}+11.8(\mathrm{c}=0.85, \mathrm{EtOH})}^{24}$

SFC: $\quad t_{R}(3 R)-7 \mathbf{b a}, 5.15(97.9 \%) ; t_{R}(3 S)-7 \mathbf{b a}, 5.58(2.1 \%)$ (OD column, $125 \mathrm{psi}, 40{ }^{\circ} \mathrm{C}$, $2.0 \% \mathrm{CH}_{3} \mathrm{OH}$ in $\mathrm{CO}_{2}, 2.5 \mathrm{~mL} / \mathrm{min}, 220 \mathrm{~nm}$ )

Analysis: $\quad \mathrm{C}_{13} \mathrm{H}_{18} \mathrm{O}_{3}(222.28)$

Calcd: $\quad$ C, $70.24 ; \quad$ H, $8.16 \%$

Found: $\quad \mathrm{C}, 70.03 ; \quad \mathrm{H}, 8.07 \%$ 


\section{Preparation of tert-Butyl 3-Hydroxy-3-(1-naphthyl)propanoate (7bb)}

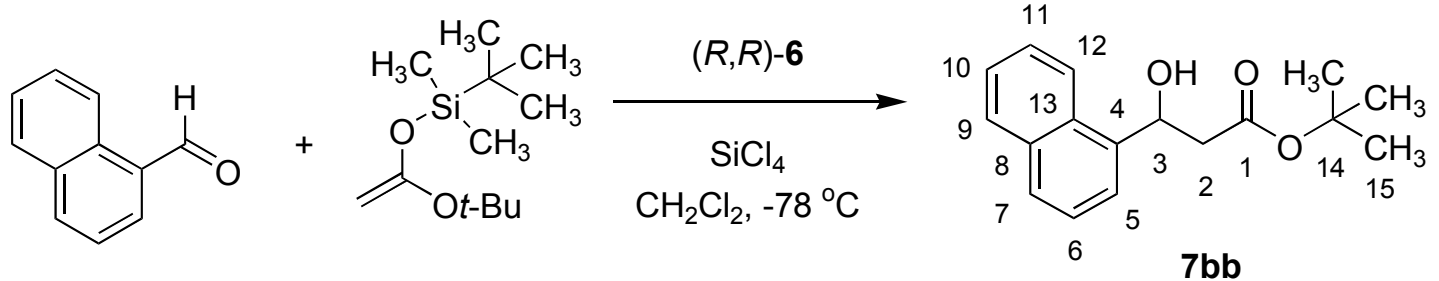

Following General Procedure 4, $270 \mu \mathrm{L}$ (2.0 mmol) of 1-napthaldehyde ${ }^{18}$ was combined with 17 $\mathrm{mg}(0.02 \mathrm{mmol}, 0.01$ equiv) of bis-phosphoramide $((R, R)-6), 250 \mu \mathrm{L}$ (2.2 mmol, 1.1 equiv) of $\mathrm{SiCl}_{4}$ and $552 \mathrm{mg}$ of $\mathbf{1 b}$ (2.4 mmol, 1.2 equiv) to yield a clear colorless oil $(480 \mathrm{mg})$. Crude ${ }^{1} \mathrm{H}$ NMR analysis indicated that a 6:1 mixture of the tert-butyl ester $\mathbf{7 b b}$ and the tertbutyldimethylsilyl ester $\mathbf{8 b}$ had formed. After additional quenching in a stirred solution of 1/1 sat. aq. $\mathrm{KF} / 1.0 \mathrm{M} \mathrm{K}_{2} \mathrm{HPO}_{4}(50 \mathrm{~mL})$, this residue was purified by Kugelrohr distillation to yield $430 \mathrm{mg}(79 \%)$ of $\mathbf{7 b b}$ as a white crystalline solid. Recrystallization from hexanes afforded colorless needles. The NMR spectral data matched that previously reported in the literature. ${ }^{17}$

Data for $7 \mathbf{b b}$ :

bp: $\quad 250{ }^{\circ} \mathrm{C}(0.5 \mathrm{mmHg}, \mathrm{ABT})$

mp: $\quad 79-79.5^{\circ} \mathrm{C}$ (hexanes)

${ }^{1} \mathrm{H}$ NMR: $\quad\left(500 \mathrm{MHz}, \mathrm{CDCl}_{3}\right)$

8.07 (d, $J=8.0,1 \mathrm{H}, \mathrm{HC}(12)), 7.90-7.86$ (m, $1 \mathrm{H}, \mathrm{HC}(9)), 7.79$ (d, $J=8.0,1 \mathrm{H}$, HC(7)), 7.70 (d, $J=7.0,1$ H, HC(5)), 7.56-7.46 (m, 3 H, HC(6,10,11)), 5.88 (dd, $J=10.0,3.0,1 \mathrm{H}, \mathrm{HC}(3)), 3.60$ (br s, $1 \mathrm{H}, \mathrm{OH}), 2.85$ (dd, $J=17.0,3.0,1 \mathrm{H}$, $H \mathrm{HC}(2)), 2.77$ (dd, $J=17.0,10.0, \mathrm{H} H \mathrm{C}(2)), 1.50$ (s, $9 \mathrm{H}, \mathrm{HC}(15))$

\section{${ }^{13} \mathrm{C} \mathrm{NMR}: \quad\left(125 \mathrm{MHz}, \mathrm{CDCl}_{3}\right)$}

172.46 (C(1)), 138.32 (C(4)), 133.97 (C(8)), 130.27 (C(13)), 129.20 (C(9)), 128.40 (C(7)), 126.43 (C(11)), 125.78 (C(10)), 125.74 (C(6)), 123.23 (C(5)), 123.12 (C(12)), 81.88 (C(14)), 67.60 (C(3)), 43.82 (C(2)), 28.37 (C(15))

IR: (thin film)

$3436(\mathrm{~m}), 2977(\mathrm{~m}), 1719$ (s)

MS: (ESI) $295\left(\mathrm{M}^{+\mathrm{Na}}, 100\right), 279$ (30), 255 (55), 199 (25)

HRMS: $\quad$ calcd for $\mathrm{C}_{17} \mathrm{H}_{20} \mathrm{O}_{3} \mathrm{Na}$ : 295.1337, found 295.1325 
TLC: $\quad R_{f} 0.3$ (Hexanes/EtOAc, 4/1) [UV(254)/KMnO 4$]$

Opt. Rot.: $[\alpha]_{D}^{24}+28.2(\mathrm{c}=2.2, \mathrm{EtOH})$

SFC: $\quad t_{R}(3 S)-7 \mathbf{b b}, 3.4(3.1 \%) ; t_{R}(3 R)-7 \mathbf{b b}, 4.4(96.9 \%)\left(\mathrm{OD}\right.$ column, $200 \mathrm{psi}, 40{ }^{\circ} \mathrm{C}$, $10.0 \% \mathrm{MeOH}$, in $\mathrm{CO}_{2}, 3.0 \mathrm{~mL} / \mathrm{min}, 220 \mathrm{~nm}$ )

Analysis: $\quad \mathrm{C}_{17} \mathrm{H}_{20} \mathrm{O}_{3}(272.34)$

Calcd: $\quad$ C, $74.97 ; \quad$ H, $7.40 \%$

Found: $\quad$ C, $74.92 ; \quad$ H, $7.47 \%$

Preparation of tert-Butyl 3-Hydroxy-4-methyl-5-phenyl-4-pentenoate (7bh)<smiles>CC(C=O)=Cc1ccccc1</smiles><smiles>C=C(O[Ga])O[Si](C)(C)C(C)(C)C</smiles><smiles>C/C(=C\c1ccccc1)C(O)CC(=O)OC(C)(C)C</smiles>

$7 \mathrm{bh}$

Following General Procedure 4, $280 \mathrm{~mL}(2.0 \mathrm{mmol}) \alpha$-methylcinnamaldehyde was combined with $17 \mathrm{mg}$ (0.02 mmol, 0.01 equiv) of bis-phosphoramide $((R, R)-6), 250 \mu \mathrm{L}(2.2 \mathrm{mmol}, 1.1$ equiv) of $\mathrm{SiCl}_{4}$ and $552 \mathrm{mg}$ of $\mathbf{1 b}(2.4 \mathrm{mmol}, 1.2$ equiv) to yield a clear colorless oil (580 $\mathrm{mg})$. Crude ${ }^{1} \mathrm{H}$ NMR analysis indicated a 2.2:1 mixture of the tert-butyl ester $7 \mathbf{b h}$ and the tertbutyldimethylsilyl ester $\mathbf{8 h}$ had formed. After additional quenching in a stirred solution of 1/1 sat. aq. $\mathrm{KF} / 1.0 \mathrm{M} \mathrm{KH}_{2} \mathrm{PO}_{4}(50 \mathrm{~mL})$, filtration through a $1 \mathrm{~cm}$ pad of $\mathrm{SiO}_{2}$ (hexanes/EtOAc, 4/1) and concentration in vacuo yielded $303 \mathrm{mg}$ (58\%) of $7 \mathbf{b h}$ as a white solid. Recrystallization from hexanes afforded fine, colorless needles.

Data for 7bh:

mp: $\quad 78-79{ }^{\circ} \mathrm{C}$ (hexanes)

${ }^{1} \mathrm{H}$ NMR: $\quad\left(400 \mathrm{MHz}, \mathrm{CDCl}_{3}\right)$

7.34 (t, $J=7.0,2$ H, HC(9)), 7.28-7.18 (m, 3 H, HC(8,10)), 6.59 (s, $1 \mathrm{H}, \mathrm{HC}(6))$,

4.59-4.51 (m, $1 \mathrm{H}, \mathrm{HC}(3)), 3.22(\mathrm{~d}, J=3.0,1 \mathrm{H}, \mathrm{OH}), 2.58(\mathrm{~d}, J=6.0,2 \mathrm{H}$, $\mathrm{HC}(2)), 1.88$ (br s, $3 \mathrm{H}, \mathrm{HC}(5)), 1.47$ (s, 9 H, HC(12))

${ }^{13} \mathrm{C} \mathrm{NMR}: \quad\left(100 \mathrm{MHz}, \mathrm{CDCl}_{3}\right)$

$172.27(\mathrm{C}(1)), 128.45(\mathrm{C}(7)), 127.65(\mathrm{C}(4)), 129.22$ (C(8)), $128.33(\mathrm{C}(9)), 126.73$ 
(C(10)), 126.06 (C(6)), 81.72 (C(11)), 73.85 (C(3)), 41.40 (C(2)), 28.35 (C(12)), $14.13(\mathrm{C}(5))$

IR: (thin film)

3435 (m), $2977(\mathrm{~m}), 1719$ (s)

MS: $\quad(\mathrm{ESI})$

$285\left(\mathrm{M}^{+\mathrm{Na}}, 17\right), 189(100)$

HRMS: $\quad$ calcd for $\mathrm{C}_{16} \mathrm{H}_{22} \mathrm{O}_{3} \mathrm{Na}$ : 285.1467, found 285.1452

TLC: $\quad R_{f} 0.3$ (hexanes/EtOAc, 4/1) [UV(254)/KMnO 4$]$

Opt. Rot.: $\quad[\alpha]_{\mathrm{D}}^{24}-23.9(\mathrm{c}=0.95$, EtOH $)$

SFC: $\quad t_{R}(3 S)-7 \mathbf{b h}, 3.4(14.5 \%) ; t_{R}(3 R)-7 \mathbf{b h}, 3.7(85.5 \%)\left(\mathrm{AD}\right.$ column, $150 \mathrm{psi}, 40{ }^{\circ} \mathrm{C}$, $5.0 \% \mathrm{MeOH}$ in $\mathrm{CO}_{2}, 3.0 \mathrm{~mL} / \mathrm{min}, 220 \mathrm{~nm}$ )

Analysis: $\quad \mathrm{C}_{16} \mathrm{H}_{22} \mathrm{O}_{3}(262.30)$

Calcd: $\quad$ C, $73.25 ; \quad H, 8.45 \%$

Found: $\quad$ C, $73.19 ; \quad$ H, $8.40 \%$

Preparation of Methyl 3-Hydroxy-5-phenylpentanoate (7bk)<smiles>C=C(OCC)O[Si](C)(C)C(C)(C)C</smiles>

A flame-dried, $25 \mathrm{~mL}, 2$-neck flask containing a solution of $17 \mathrm{mg}$ (0.02 mmol, 0.01 equiv) of bis-phosphormaide $((R, R)-6)$ in $\mathrm{CH}_{2} \mathrm{Cl}_{2}(10 \mathrm{~mL})$ was cooled to $-78^{\circ} \mathrm{C}$ and $260 \mu \mathrm{L}(2.0 \mathrm{mmol})$ of hydrocinnamaldehyde and $70 \mu \mathrm{L}(0.4 \mathrm{mmol}, 0.2$ equiv $)$ of DIPEA were added. To the resulting solution, $250 \mu \mathrm{L}$ (2.2 mmol, 1.1 equiv) of $\mathrm{SiCl}_{4}$ was added and the reaction was allowed to stir at $-78{ }^{\circ} \mathrm{C}$ for $5 \mathrm{~min}$. Then, $552 \mathrm{mg}(2.4 \mathrm{mmol}, 1.2 \mathrm{equiv})$ of $\mathbf{1 b}$ was added dropwise (over $\left.1 \mathrm{~min}\right)$ to the reaction mixture. The resulting mixture was allowed to stir at $-78{ }^{\circ} \mathrm{C}$ for $6 \mathrm{~h}$ whereupon the cold reaction mixture was poured into a rapidly stirring solution of saturated aqueous $\mathrm{NaHCO}_{3}$ $(50 \mathrm{~mL})$ and the resulting mixture was stirred for $30 \mathrm{~min}$ at room temperature. The solution was then filtered through Celite and the filter cake was washed with $\mathrm{CH}_{2} \mathrm{Cl}_{2}(100 \mathrm{~mL})$. The aqueous layer was washed with $\mathrm{CH}_{2} \mathrm{Cl}_{2}(50 \mathrm{~mL})$ and the combined organics were dried over $\mathrm{K}_{2} \mathrm{CO}_{3}(5 \mathrm{~g})$ 
and concentrated in vacuo to give a colorless oil $(280 \mathrm{mg})$. Crude ${ }^{1} \mathrm{H}$ NMR analysis indicated that a 95:5 mixture of the tert-butyl ester $\mathbf{7 b k}$ and the tert-butyldimethylsilyl ester $\mathbf{8 k}$ had formed. However, the tert-butyldimethylsilyl ester $\mathbf{8 k}$ was found to hydrolyse rapidly in the $\mathrm{NaHCO}_{3}$ solution and therefore this may not be representative of the true ratio. The residue was purified by column chromatography (hexanes/EtOAc, 3/1) to yield $232 \mathrm{mg}(46 \%)$ of $7 \mathbf{b k}$ as a colorless oil.

Data for $7 \mathbf{b k}$ :

1 NMR: $\quad\left(500 \mathrm{MHz}, \mathrm{CDCl}_{3}\right)$

7.31-7.27 (m, 2 H, HC(7)), 7.22-7.17 (m, 3 H, HC(8,9)), 4.00-3.94 (m, $1 \mathrm{H}$, HC(3)), 3.22 (br s, $1 \mathrm{H}, \mathrm{OH}), 2.82$ (ddd, $J=13.5,10.0,5.0,1 \mathrm{H}, H \mathrm{HC}(5)), 2.70$ (ddd, $J=13.5,9.5,6.5,1 \mathrm{H}, \mathrm{H} H \mathrm{C}(5)), 2.43$ (dd, $J=17.0,4.0,1 \mathrm{H}, H \mathrm{HC}(2)$ ), 2.36 (dd, $J=16.5,9.0,1 \mathrm{H}, \mathrm{H} H \mathrm{C}(2))$ ) 1.87-1.79 (m, $1 \mathrm{H}, H \mathrm{HC}(4))$ ), 1.75-1.68 (m, $1 \mathrm{H}, \mathrm{H} H \mathrm{C}(4)), 1.46$ (s, $9 \mathrm{H}, \mathrm{HC}(11))$

${ }^{13} \mathrm{C} \mathrm{NMR}: \quad\left(125 \mathrm{MHz}, \mathrm{CDCl}_{3}\right)$

$172.80(\mathrm{C}(1)), 142.12(\mathrm{C}(6)), 128.71(\mathrm{C}(7)), 128.64(\mathrm{C}(8)), 126.08(\mathrm{C}(9)), 81.60$ (C(1)), $67.71(\mathrm{C}(3)), 42.44(\mathrm{C}(2)), 38.28(\mathrm{C}(5)), 31.96(\mathrm{C}(4)), 28.31(\mathrm{C}(11))$

IR: (thin film)

$3436(\mathrm{~m}), 2929(\mathrm{~m}), 1718(\mathrm{~s})$

MS: $\quad(\mathrm{ESI})$

$273\left(\mathrm{M}^{+\mathrm{Na}}, 50\right), 157$ (100), $195(55), 177$ (30)

HRMS: calcd for $\mathrm{C}_{15} \mathrm{H}_{22} \mathrm{O}_{3} \mathrm{Na}$ : 273.1467, found 273.1465

TLC: $\quad R_{f} 0.5$ (Hexanes/EtOAc, 3/1) [UV(254)/ $\left.\mathrm{KMnO}_{4}\right]$

Opt. Rot.: $\quad[\alpha]_{\mathrm{D}}^{24}-14.8(\mathrm{c}=0.45, \mathrm{EtOH})$

SFC: $\quad t_{R}(3 S)-7 \mathbf{b k}, 3.0(96.9 \%) ; t_{R}(3 R)-7 \mathbf{b k}, 3.6(3.1 \%)\left(\mathrm{OD}\right.$ column, $150 \mathrm{psi}, 40{ }^{\circ} \mathrm{C}$, $5.0 \% \mathrm{MeOH}$ in $\mathrm{CO}_{2}, 3.0 \mathrm{~mL} / \mathrm{min}, 220 \mathrm{~nm}$ )

Analysis: $\quad \mathrm{C}_{15} \mathrm{H}_{22} \mathrm{O}_{3}(250.30)$

Calcd: $\quad$ C, $71.97 ; \quad \mathrm{H}, 8.86 \%$

Found: $\quad \mathrm{C}, 71.77 ; \quad \mathrm{H}, 8.93 \%$ 


\section{General Procedure 5. Addition of propionate-derived Silyl Ketene Acetals to Aromatic} Aldehydes: Preparation of tert-Butyl 3-Hydroxy-2-methyl-3-phenylpropanoate ((2S,3R)9ea)

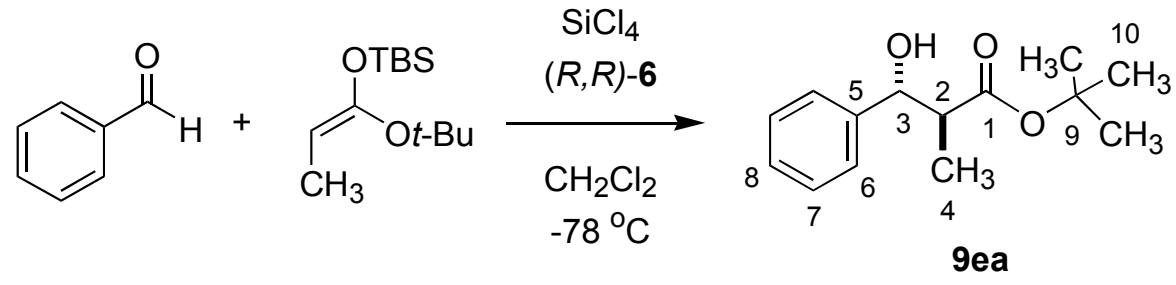

A flame-dried, 10-mL, 2-neck flask containing a solution of $8.4 \mathrm{mg}(0.01 \mathrm{mmol}, 0.01$ equiv) of bis-phosphoramide $((R, R)-6)$ in $\mathrm{CH}_{2} \mathrm{Cl}_{2}(5 \mathrm{~mL})$ was cooled to $-78{ }^{\circ} \mathrm{C}$ under nitrogen in a dry ice/isopropanol bath and then $102 \mu \mathrm{L}(1.0 \mathrm{mmol})$ of benzaldehyde was added. To the resulting solution was added $123 \mu \mathrm{L}\left(1.1 \mathrm{mmol}, 1.1\right.$ equiv) of $\mathrm{SiCl}_{4}$ and the reaction mixture was allowed to stir at $-78^{\circ} \mathrm{C}$ for $5 \mathrm{~min}$ in a dry ice/isopropanol bath. Then $293 \mathrm{mg}$ (1.2 mmol, 1.2 equiv) of (E)-1-[(tert-butoxy)-propenyl]-tert-butyl-dimethylsilane (2e) was added dropwise (over 5 min) to the reaction mixture. The resulting mixture was allowed to stir at $-78{ }^{\circ} \mathrm{C}$ for $3 \mathrm{~h}$ in a dry ice/isopropanol bath whereupon the cold reaction mixture was poured into a rapidly stirring $1 / 1$ sat. aq. $\mathrm{KF} / 1.0 \mathrm{M} \mathrm{KH}_{2} \mathrm{PO}_{4}$ solution $(20 \mathrm{~mL})$. This biphasic mixture was stirred vigorously for 1 $\mathrm{h}$ before filtration through Celite. The aqueous layer was washed with $\mathrm{CH}_{2} \mathrm{Cl}_{2}(3 \times 50 \mathrm{~mL})$. The combined organic extracts were washed with brine $(50 \mathrm{~mL})$, dried over $\mathrm{Na}_{2} \mathrm{SO}_{4}(2 \mathrm{~g})$, filtered and the filtrate was concentrated in vacuo. The residue was purified by Kugelrohr distillation to yield $217 \mathrm{mg}$ (93\%) of tert-butyl 3-hydroxy-2-methyl-3-phenyl propanoate $((2 S, 3 R)-9$ ea) as a colorless oil. NMR spectral data matched that previously reported in the literature. ${ }^{19}$

Data for $(2 S, 3 R)-9 \mathbf{e a}:$

bp: $150{ }^{\circ} \mathrm{C}(0.01 \mathrm{mmHg}, \mathrm{ABT})$

${ }^{1} \mathrm{H}$ NMR: $\quad\left(499 \mathrm{MHz}, \mathrm{CDCl}_{3}\right)$

7.40-7.20 (m, $5 \mathrm{H}, \mathrm{HC}(6,7,8)), 4.70(\mathrm{dd}, J=7.9,4.9,1 \mathrm{H}, \mathrm{HC}(3)), 3.19$ (d, $J=$ 4.9, $1 \mathrm{H}, \mathrm{OH}), 2.70$ (pent, $J=7.3,1 \mathrm{H}, \mathrm{HC}(2)), 1.44$ (s, $\left.9 \mathrm{H}, \mathrm{H}_{3} \mathrm{C}(10)\right), 1.01$ (d, $J$ $\left.=7.3,3 \mathrm{H}, \mathrm{H}_{3} \mathrm{C}(4)\right)$ 


\section{${ }^{13} \mathrm{C}$ NMR: $\quad\left(125 \mathrm{MHz}, \mathrm{CDCl}_{3}\right)$}

$175.3(\mathrm{C}(1)), 141.8(\mathrm{C}(5)), 128.3(\mathrm{C}(7)), 127.8$ (C(8)), $126.3(\mathrm{C}(6)), 81.2(\mathrm{C}(9))$,

76.3 (C(3)), $47.68(\mathrm{C}(2)), 27.9$ (C(10)), 14.6 (C(4))

IR: (neat)

3455 (w), 2978 (s), 2936 (m), 1727 (s), 1456 (m), 1368 (s), 1253 (m), 1154 (s), $1021(\mathrm{~m}), 701(\mathrm{~m})$

MS: $\quad(\mathrm{FAB})$

$237\left(\mathrm{M}^{+}, 6\right), 181(11), 163(25), 155$ (43), $135(50), 119$ (100)

TLC: $\quad R_{f} 0.35$ (hexanes/EtOAc, 8/2) [UV(254)/KMnO $\mathrm{KM}_{4}$

SFC: $\quad t_{R}(2 S, 3 R)-9$ ea, $2.32(>99.9 \%) ; t_{R}(2 R, 3 S)$-9ea, $2.81 \mathrm{~min}(<0.1 \%)$ (Chiralpak AS, $150 \mathrm{psi}, 40{ }^{\circ} \mathrm{C}, 1.0 \% \mathrm{CH}_{3} \mathrm{OH}$ in $\mathrm{CO}_{2}, 3.5 \mathrm{~mL} / \mathrm{min}, 220 \mathrm{~nm}$ )

Opt. Rot.: $\quad[\alpha]_{\mathrm{D}}^{24}+36.70(\mathrm{c}=1.0, \mathrm{EtOH})$

Analysis: $\quad \mathrm{C}_{14} \mathrm{H}_{20} \mathrm{O}_{3}(230.26)$

Calcd: $\quad$ C, $71.16 ; \quad \mathrm{H}, 8.53 \%$

Found: $\quad$ C, $70.93 ; \quad H, 8.46 \%$

\section{Preparation of tert-Butyl 3-Hydroxy-2-methyl-3-(1-naphthyl)propanoate (9eb)}

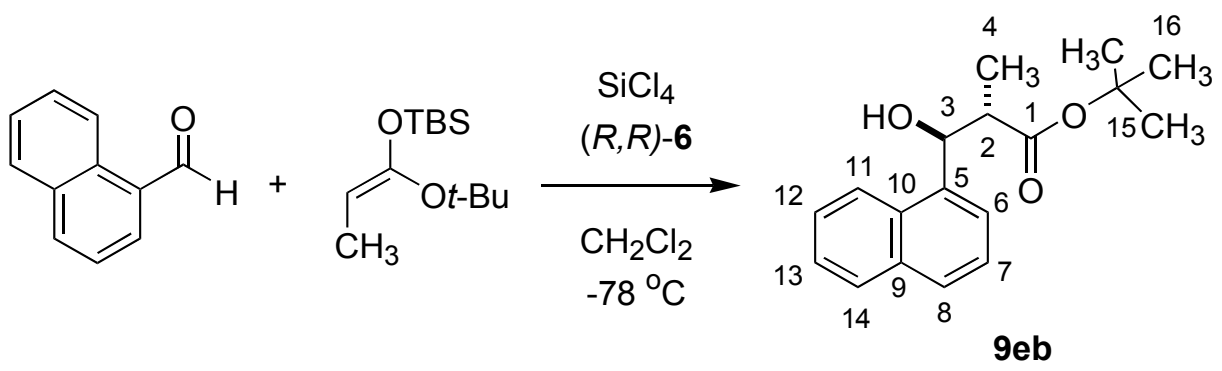

Following General Procedure 5, $136 \mu \mathrm{L}(1.0 \mathrm{mmol})$ of 1-naphthaldehyde was combined with 8.4 $\mathrm{mg}(0.01 \mathrm{mmol}, 0.01$ equiv) of bis-phosphoramide $((R, R)-6), 123 \mu \mathrm{L}(1.1 \mathrm{mmol}, 1.1$ equiv) of $\mathrm{SiCl}_{4}$, and $293 \mathrm{mg}$ (1.2 mmol, 1.2 equiv) of (E)-1-[(tert-butoxy)-propenyl]-tert-butyldimethylsilane (2e) to yield, after Kugelrohr distillation, $280 \mathrm{mg}(98 \%)$ of tert-butyl 3-hydroxy2-methyl-3-(1-naphthyl) propanoate (9eb) as a colorless wax. NMR spectral data matched that previously reported in the literature. ${ }^{20}$ 


\section{Data for 9eb:}

bp: $\quad 210^{\circ} \mathrm{C}(0.01 \mathrm{mmHg}, \mathrm{ABT})$

근 $\quad\left(499 \mathrm{MHz}, \mathrm{CDCl}_{3}\right)$

$8.2(\mathrm{~d}, J=8.1,1 \mathrm{H}, \mathrm{HC}(11)), 7.88(\mathrm{~d}, J=7.9,1 \mathrm{H}, \mathrm{HC}(14)), 7.8(\mathrm{~d}, J=8.4,1 \mathrm{H}$, $\mathrm{HC}(8)), 7.57$ (d, $J=7.5,1 \mathrm{H}, \mathrm{HC}(6)), 7.51-7.45$ (m, $3 \mathrm{H}, \mathrm{HC}(7,12,13)), 5.48$ (dd, $J=7.5,5.2,1 \mathrm{H}, \mathrm{HC}(3)), 3.52$ (d, $J=5.2,1 \mathrm{H}, \mathrm{OH}), 3.04$ (pent, $J=7.3,1 \mathrm{H}$, $\mathrm{HC}(2)), 1.44$ (s, $\left.9 \mathrm{H}, \mathrm{H}_{3} \mathrm{C}(16)\right), 1.06$ (d, $\left.J=7.1,3 \mathrm{H}, \mathrm{H}_{3} \mathrm{C}(4)\right)$

${ }^{13} \mathrm{C} \mathrm{NMR}: \quad\left(125 \mathrm{MHz}, \mathrm{CDCl}_{3}\right)$

175.59 (C(1)), 137.44 (C(5)), 133.82 (C(9)), 130.91 (C(10)), 128.88 (C(14)), 128.39 (C(8)), 126.02 (C(12)), 125.49 (C(13)), 125.23 (C(7)), 124.51 (C(6)), 123.51 (C(11)), 81.29 (C(15)), 73.57 (C(3)), 47.05 (C(2)), 28 (C(16)), 15.29 $(\mathrm{C}(4))$

IR: $\quad\left(\mathrm{CHCl}_{3}\right)$

3606 (w), $3474(\mathrm{w}), 2981$ (m), 1712 (s), 1369 (s), 1154 (s), 780 (m)

MS: $\quad(\mathrm{EI}, 70 \mathrm{eV})$

$286\left(\mathrm{M}^{+}, 8\right), 230(5), 213(9), 151$ (100), $129(50), 73$ (12)

TLC: $\quad R_{f} 0.5$ (hexanes/EtOAc, 8/2) [UV(254)/KMnO $\mathrm{KM}_{4}$

Opt. Rot.: $\quad[\alpha]_{\mathrm{D}}^{24}+28.61(\mathrm{c}=1.0, \mathrm{EtOH})$

SFC: $\quad t_{R}(2 S, 3 R)-9 \mathrm{eb}, 4.11(2.8 \%) ; t_{R}(2 R, 3 S)-9 \mathrm{eb}, 4.52 \mathrm{~min}(97.2 \%)$ (Chiralpak AD, $150 \mathrm{psi}, 40{ }^{\circ} \mathrm{C}, 5.0 \% \mathrm{CH}_{3} \mathrm{OH}$ in $\mathrm{CO}_{2}, 3.0 \mathrm{~mL} / \mathrm{min}, 220 \mathrm{~nm}$ )

Analysis: $\quad \mathrm{C}_{18} \mathrm{H}_{22} \mathrm{O}_{3}(286.37)$
Calcd:
C, 75.50;
$\mathrm{H}, 7.74 \%$
Found:
C, 75.57;
H, $7.90 \%$ 


\section{Preparation of tert-Butyl 3-Hydroxy-2-methyl-3-(2-naphthlyl)propanoate (9ec)}

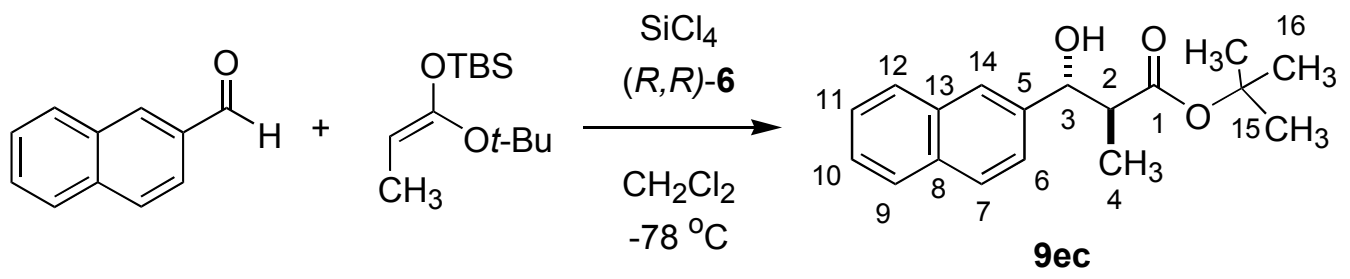

Following General Procedure 5, $156 \mathrm{mg}(1.0 \mathrm{mmol})$ of 2-naphthaldehyde was combined with 8.4 $\mathrm{mg}(0.01 \mathrm{mmol}, 0.01$ equiv) of bis-phosphoramide $((R, R)-6), 123 \mu \mathrm{L}(1.1 \mathrm{mmol}, 1.1$ equiv) of $\mathrm{SiCl}_{4}$, and $293 \mathrm{mg}$ (1.2 mmol, 1.2 equiv) of (E)-1-[(tert-butoxy)-propenyl]-tert-butyldimethylsilane (2e) to yield, after Kugelrohr distillation, $271 \mathrm{mg}(95 \%)$ of tert-butyl 3-hydroxy2-methyl-3-(2-naphthlyl) propanoate (9ec) as a colorless wax.

Data for 9ec:

bp: $\quad 210^{\circ} \mathrm{C}(0.01 \mathrm{mmHg}, \mathrm{ABT})$

${ }^{1} \mathrm{H}$ NMR: $\quad\left(499 \mathrm{MHz}, \mathrm{CDCl}_{3}\right)$

7.83 (m, 4 H, H(Ar)), 7.48 (m, 3 H, H(Ar)), 4.87 (d, J= 8.2, 1 H, HC(3)), 3.33 (br s, $1 \mathrm{H}, \mathrm{OH}), 2.82$ (pent, $J=7.2,1 \mathrm{H}, \mathrm{HC}(2)), 1.44$ (s, $\left.9 \mathrm{H}, \mathrm{H}_{3} \mathrm{C}(16)\right), 1.04$ (d, $J=$ $\left.7.2,3 \mathrm{H}, \mathrm{H}_{3} \mathrm{C}(4)\right)$

${ }^{13} \mathrm{C} \mathrm{NMR}: \quad\left(125 \mathrm{MHz}, \mathrm{CDCl}_{3}\right)$

175.3 (C(1)), 139.2 (C(5)), 133.13 (C(13)), 133.10 (C(8)), 128.2 (C(9)), 127.9 (C(12)), 127.6 (C(6)), 126.1 (C(7)), 125.9 (C(14)), 125.8 (C(11)), 124.3 (C(10)), 81.27 (C(15)), 76.5 (C(3)), 47.89 (C(2)), 27.98 (C(16)), 14.72 (C(4))

IR: (neat)

3343 (w), 2977 (s), 2935 (m), 1724 (s), 1711 (s), 1457 (s), 1368 (s), 1255 (s), 1155 (s), 1026 (s), 821 (m), 748 (m)

MS: $\quad(\mathrm{EI}, 70 \mathrm{eV})$

$286\left(\mathrm{M}^{+}, 8\right), 229(10), 193$ (10), 151 (100), 129 (51), 73 (12)

TLC: $\quad R_{f} 0.44$ (hexanes/EtOAc, $\left.8 / 2\right)\left[\mathrm{UV}(254) / \mathrm{KMnO}_{4}\right]$

Opt. Rot.: $\quad[\alpha]_{\mathrm{D}}^{24}+21.37(\mathrm{c}=1, \mathrm{EtOH})$

SFC: $\quad t_{R}(2 S, 3 R)-9 e c, 3.26(99.6 \%) ; t_{R}(2 R, 3 S)$-9ec, $3.67 \mathrm{~min}(0.4 \%)$ (Chiralpak AS, 150 psi, $40{ }^{\circ} \mathrm{C}, 5.0 \% \mathrm{CH}_{3} \mathrm{OH}$ in $\left.\mathrm{CO}_{2}, 2.5 \mathrm{~mL} / \mathrm{min}, 220 \mathrm{~nm}\right)$ 
Analysis: $\quad \mathrm{C}_{18} \mathrm{H}_{22} \mathrm{O}_{3}(286.37)$

$\begin{array}{lll}\text { Calcd: } & \text { C, 75.50; } & \text { H, 7.74\% } \\ \text { Found: } & \text { C, 75.41; } & \text { H, 7.78\% }\end{array}$

Preparation of tert-Butyl 3-Hydroxy-3-(4-methoxyphenyl)-2-methylpropanoate (9ee)

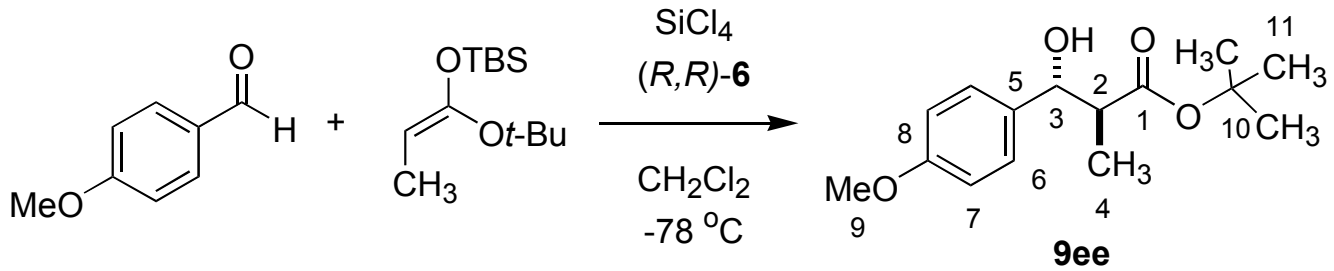

Following General Procedure 5, $122 \mu \mathrm{L}(1.0 \mathrm{mmol})$ of $p$-anisaldehyde was combined with 8.4 $\mathrm{mg}(0.01 \mathrm{mmol}, 0.01$ equiv.) of bis-phosphoramide $((R, R)-6), 123 \mu \mathrm{L}(1.1 \mathrm{mmol}, 1.1 \mathrm{equiv})$ of $\mathrm{SiCl}_{4}$, and $293 \mathrm{mg}$ (1.2 mmol, 1.2 equiv) of (E)-1-[(tert-butoxy)-propenyl]-tert-butyldimethylsilane (2e) to yield, after Kugelrohr distillation, $233 \mathrm{mg}(88 \%)$ of tert-butyl 3-hydroxy3-(4-methoxyphenyl)-2-methyl propanoate (9ee) as a colorless oil.

Data for 9ee:

bp: $\quad 175^{\circ} \mathrm{C}(0.01 \mathrm{mmHg}, \mathrm{ABT})$

${ }^{1} \mathrm{H}$ NMR: $\quad\left(500 \mathrm{MHz}, \mathrm{CDCl}_{3}\right)$

7.16 (m, 2 H, HC(6)), 6.77 (m, 2 H, HC(7)), 4.55 (d, J = 8.4, 1 H, HC(3)), 2.99 (s, $1 \mathrm{H}, \mathrm{OH}), 2.57$ (pent, $J=7.3,1 \mathrm{H}, \mathrm{HC}(2)), 1.35$ (s, $\left.9 \mathrm{H}, \mathrm{H}_{3} \mathrm{C}(11)\right), 0.88$ (d, $J=$ $\left.7.3,3 \mathrm{H}, \mathrm{H}_{3} \mathrm{C}(4)\right)$

${ }^{13} \mathrm{C} \mathrm{NMR}: \quad\left(125 \mathrm{MHz}, \mathrm{CDCl}_{3}\right)$

175.43 (C(1)), 159.2 (C(8)), 133.98 (C(5)), 127.84 (C(6)), 113.72 (C(7)), 81.08 (C(10), 75.93 (C(3)), 55.23 (C(9)), 47.81 (C(2)), 27.99 (C(11)), 14.60 (C(4))

IR: (neat) 3459 (w), 2977 (m), 29369 (m), 1726 (s), 1612 (m), 1513 (s), 1460 (m), 1368 (s), 1251 (s), 1154 (s), 1035 (m), 833 (m)

MS.: $\quad(\mathrm{EI}, 70 \mathrm{eV})$ $266\left(\mathrm{M}^{+}, 8\right), 209$ (3), 193 (10), 134 (100), 108 (9), 76 (7)

TLC: $\quad R_{f} 0.34$ (hexanes/EtOAc, 8/2) [UV(254)/KMnO $\left.{ }_{4}\right]$ 
Opt. Rot.: $\quad[\alpha]_{\mathrm{D}}^{24}+24.59(\mathrm{c}=1, \mathrm{EtOH})$

SFC: $\quad t_{R}(2 S, 3 R)-9 e e, 2.46(99.2 \%) ; t_{R}(2 R, 3 S)$-9ee, $3.05 \mathrm{~min}(0.8 \%)$ (Chiralpak AS, 150 psi, $40{ }^{\circ} \mathrm{C}, 5.0 \% \mathrm{CH}_{3} \mathrm{OH}$ in $\left.\mathrm{CO}_{2}, 2.5 \mathrm{~mL} / \mathrm{min}, 220 \mathrm{~nm}\right)$

Analysis: $\quad \mathrm{C}_{15} \mathrm{H}_{22} \mathrm{O}_{4}(266.33)$
Calcd:
C, 67.64;
H, $8.33 \%$
Found:
C, 67.58;
$\mathrm{H}, 8.44 \%$

Preparation of tert-Butyl 3-Hydroxy-2-methyl-3-(4-trifluoromethylphenyl)propanoate (9ef)

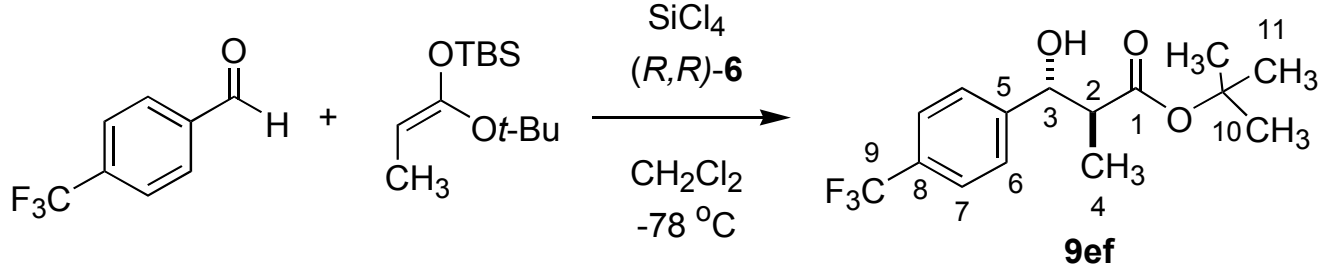

Following General Procedure 5, $136 \mu \mathrm{L}(1.0 \mathrm{mmol})$ of 4-trifluoromethylbenzaldehyde was combined with $8.4 \mathrm{mg}(0.01 \mathrm{mmol}, 0.01$ equiv) of bis-phosphoramide $((R, R)-6), 123 \mu \mathrm{L}(1.1$ mmol, 1.1 equiv) of $\mathrm{SiCl}_{4}$, and $293 \mathrm{mg}$ (1.2 mmol, 1.2 equiv) of (E)-1-[(tert-butoxy)-propenyl]tert-butyl-dimethylsilane (2e) to yield, after Kugelrohr distillation, 284 mg (93\%) of tert-butyl 3hydroxy-2-methyl-3-(4-trifluoromethylphenyl) propanoate (9ef) as a white crystalline solid.

Data for 9ef:

mp: $\quad 84-86{ }^{\circ} \mathrm{C}$

매 NMR: $\quad\left(399 \mathrm{MHz}, \mathrm{CDCl}_{3}\right)$

7.53 (m, 4 H, HC(6,7)), 4.57 (d, $J=7.56,1 \mathrm{H}, \mathrm{HC}(3)), 3.48$ (s, $1 \mathrm{H}, \mathrm{OH}), 2.69$

(pent, $J=7.32,1 \mathrm{H}, \mathrm{HC}(2)), 1.41\left(\mathrm{~s}, 9 \mathrm{H}, \mathrm{H}_{3} \mathrm{C}(11)\right), 1.06(\mathrm{~d}, J=7.08,3 \mathrm{H}$,

$\left.\mathrm{H}_{3} \mathrm{C}(4)\right)$

${ }^{13} \mathrm{C} \mathrm{NMR}: \quad\left(100 \mathrm{MHz}, \mathrm{CDCl}_{3}\right)$

$175.24(\mathrm{C}(1)), 146.20(\mathrm{C}(5)), 130.30$ (q, $J=32.04, \mathrm{C}(8)), 127.17$ (C(6)), 125.52

(C(7)), 124.34 (q, $J=271.6, \mathrm{C}(9)), 81.63$ (C(10)), 75.60 (C93)), 47.38 (C(2)), $27.93(\mathrm{C}(11)), 14.63(\mathrm{C}(4))$ 
${ }^{19}$ F NMR: $\quad\left(376 \mathrm{MHz}, \mathrm{CDCl}_{3}\right)$

$-62.89\left(\mathrm{t}, 3 \mathrm{~F}, \mathrm{~F}_{3} \mathrm{C}(9)\right)$

IR: $\quad\left(\mathrm{CHCl}_{3}\right)$

3492 (w), 3009 (w), 2982 9w), 2938 (w), 1707 (s), 1369 (m), 1166 (s), 1131 (s)

$1068(\mathrm{~m}), 8449(\mathrm{~m})$

MS.: $\quad(\mathrm{EI}, 70 \mathrm{eV})$

$305\left(\mathrm{M}^{+1}, 2\right), 248$ (13), 229 (16), 175 (60), 130 (32), 73 (59)

TLC: $\quad R_{f} 0.48$ (hexanes/EtOAc, $\left.8 / 2\right)\left[\mathrm{UV}(254) / \mathrm{KMnO}_{4}\right]$

Opt. Rot.: $\quad[\alpha]_{\mathrm{D}}^{24}+39.50(\mathrm{c}=1, \mathrm{EtOH})$

SFC: $\quad t_{R}(2 S, 3 R)-9$ ef, $2.44(96.0 \%) ; t_{R}(2 R, 3 S)-9$ ef, $3.08 \mathrm{~min}(4.0 \%)$ (Whelk-O1, $150 \mathrm{psi}$, $40{ }^{\circ} \mathrm{C}, 1.0 \% \mathrm{CH}_{3} \mathrm{OH}$ in $\left.\mathrm{CO}_{2}, 3.5 \mathrm{~mL} / \mathrm{min}, 220 \mathrm{~nm}\right)$

Analysis: $\quad \mathrm{C}_{15} \mathrm{H}_{19} \mathrm{~F}_{3} \mathrm{O}_{3}(304.30)$

Calcd: $\quad$ C, 59.20; H, 6.29; $\quad$ F, $18.73 \%$

Found: $\quad$ C, 59.05; $\quad \mathrm{H}, 6.21 ; \quad \mathrm{F}, 18.61 \%$

Preparation of tert-Butyl 3-(2-Furyl)-3-hydroxy-2-methylpropanoate (9ei)

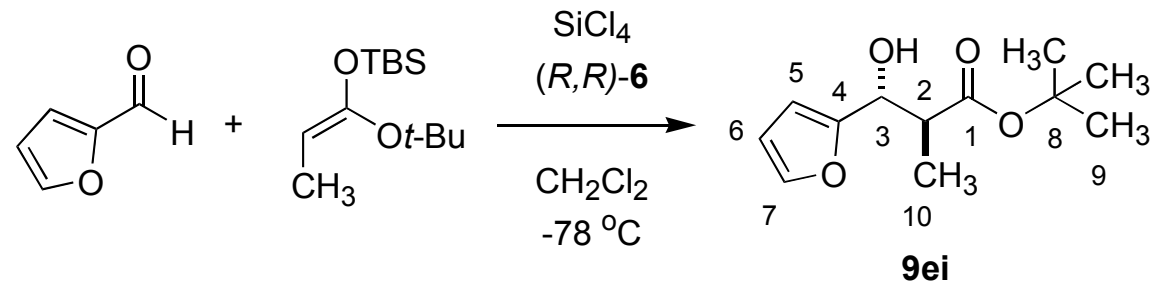

Following General Procedure 5, $100 \mu \mathrm{L}$ of 2-furaldehdye 5i (1.0 mmol) was combined with 8.4 $\mathrm{mg}(0.01 \mathrm{mmol}, 0.01$ equiv) of bis-phosphoramide $((R, R)-6), 123 \mu \mathrm{L}(1.1 \mathrm{mmol}, 1.1$ equiv) of $\mathrm{SiCl}_{4}$, and $293 \mathrm{mg}$ (1.2 mmol, 1.2 equiv) of (E)-1-[(tert-butoxy)-propenyl]-tert-butyldimethylsilane (2e) to yield, after silica gel column chromatography (hexanes/EtOAc, 9/1), 217 mg (96\%) of tert-butyl 3-(2-furyl)-3-hydroxy-2-methyl propanoate 9ei as a colorless oil.

Data for 9ei:

bp: $\quad 150{ }^{\circ} \mathrm{C}$ ABT $(0.01 \mathrm{mmHg})$ 
${ }^{1} \mathrm{H}$ NMR: $\quad\left(400 \mathrm{MHz}, \mathrm{CDCl}_{3}\right)$

$7.37(\mathrm{~d}, J=1.7,1 \mathrm{H} \mathrm{HC(7)),} 6.33(\mathrm{dd}, J=3.2,1.7,1 \mathrm{H}, \mathrm{HC}(6)), 6.27$ (d, $J=3.21$

$\mathrm{H}, \mathrm{HC}(5)), 4.72$ (t, $J=7.1,1 \mathrm{H}, \mathrm{HC}(3)), 3.27$ (d, $J=6.6,1 \mathrm{H}, \mathrm{OH}), 2.90$ (pent, $J=$ 7.1, $1 \mathrm{H}, \mathrm{HC}(2)), 1.45$ (s, $\left.9 \mathrm{H}, \mathrm{H}_{3} \mathrm{C}(9)\right), 1.01$ (d, $J=7.1,3 \mathrm{H} \mathrm{H}_{3} \mathrm{C}(10)$ )

${ }^{13} \mathrm{C} \mathrm{NMR}: \quad\left(100 \mathrm{MHz}, \mathrm{CDCl}_{3}\right)$

174.94 (C(1)), 154.53 (C(4)), 142.13 (C(7)), 110.12 (C(6)), 107.20 (C(6)), 81.34 (C(8)), 69.89 (C(3)), 45.05 (C(2)), 27.98 (C(9)), 14.35 (C(10))

IR: (neat)

3454 (m), 2978 (s), 2937 (m), 1730 (s), 1505 (w), 1458 (m), 1368 (s), 1256 (m), $1153(\mathrm{~s}), 1010(\mathrm{~m})$

MS: $\quad(\mathrm{EI}, 70 \mathrm{eV})$

$$
226\left(\mathrm{M}^{+}, 3\right), 209(5), 170(31), 153 \text { (35), } 97 \text { (100), } 57 \text { (25) }
$$

Opt. Rot.: $\quad+28.12(\mathrm{c}=1.0, \mathrm{EtOH})$

TLC: $\quad R_{f} 0.26$ (hexanes/EtOAc, 9/1) [UV(254)/KMnO $\mathrm{KM}_{4}$

SFC: $\quad t_{R}(2 S, 3 R)-9$ ei, $2.26(96.9 \%) ; t_{R}(2 R, 3 S)-9$ ei, 2.85 (3.1\%) (Chiralpak AS, 125 psi, $40{ }^{\circ} \mathrm{C}, 0.5 \% \mathrm{CH}_{3} \mathrm{OH}$ in $\mathrm{CO}_{2}, 3.5 \mathrm{~mL} / \mathrm{min}, 220 \mathrm{~nm}$ )

Analysis: $\quad \mathrm{C}_{12} \mathrm{H}_{18} \mathrm{O}_{4}(226.27)$

Calcd: $\quad$ C, 63.70; $\quad$ H, 8.02\%

Found; $\quad$ C, 63.50; H, 8.12\%

\section{Preparation of tert-Butyl 3-(2-Thienyl)-3-hydroxy-2-methylpropanoate (9el)}

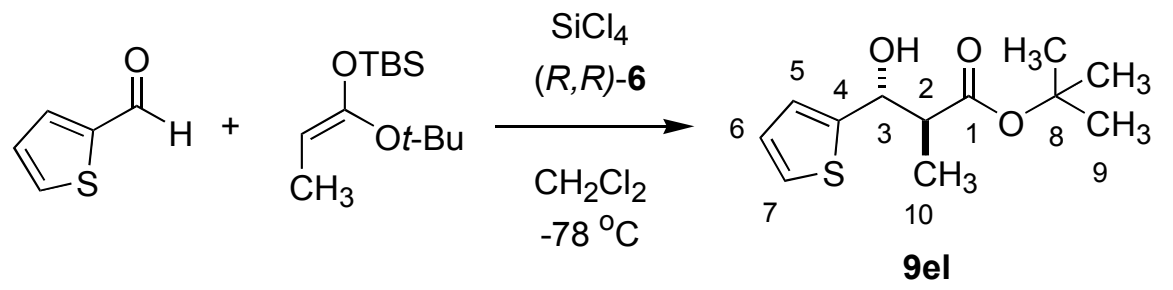

Following General Procedure 5, $8.4 \mathrm{mg}$ of bis-phosphoramide $(R, R)-6$ (0.01 mmol, 0.01 equiv) was combined with $93 \mu \mathrm{L}$ of 2-thiophene carboxaldehyde 51 (1.0 mmol), $123 \mu \mathrm{L}$ of $\mathrm{SiCl}_{4}(1.1$ mmol, 1.1 equiv) and $293 \mathrm{mg}$ of $2 \mathrm{e}(1.2 \mathrm{mmol}, 1.2$ equiv) to yield, after silica gel column 
chromatography (hexanes/EtOAc, 9/1), $218 \mathrm{mg}$ (90 \%) of tert-butyl 3-(2-thiophenyl)-3-hydroxy2-methyl propanoate 9el as a colorless oil.

Data for 9el:

bp: $\quad 150{ }^{\circ} \mathrm{C}$ ABT $(0.01 \mathrm{mmHg})^{21}$

${ }^{1} \mathrm{H}$ NMR: $\quad\left(400 \mathrm{MHz}, \mathrm{CDCl}_{3}\right)$

7.27 ( $\mathrm{m}, 1 \mathrm{H}, \mathrm{HC}(7)), 6.97$ (m, $2 \mathrm{H}, \mathrm{HC}(5,6)), 4.95$ (dd, $J=7.1,5.8,1 \mathrm{H} \mathrm{HC}(3))$, 3.44 (d, $J=5.8,1 \mathrm{H}, \mathrm{OH}$ ), 2.77 (pent, $J=7.1,1 \mathrm{H}, \mathrm{HC}(2)$ ), 1.44 (s, $9 \mathrm{H}, \mathrm{H}_{3} \mathrm{C}(9)$ ), $1.12\left(\mathrm{~s}, J=7.3,3 \mathrm{H}, \mathrm{H}_{3} \mathrm{C}(10)\right)$

${ }^{13} \mathrm{C}$ NMR: $\quad\left(100 \mathrm{MHz}, \mathrm{CDCl}_{3}\right)$ $174.98(\mathrm{C}(1)), 126.50(\mathrm{C}(4)), 125.00(\mathrm{C}(7)), 124.90(\mathrm{C}(6)), 124.60(\mathrm{C}(5)), 81.46$ (C(3)), $72.33(\mathrm{C}(8)), 47.88(\mathrm{C}(2)), 27.98(\mathrm{C}(9)), 14.69(\mathrm{C}(10))$

IR: (neat) $3463(\mathrm{~m}), 2978(\mathrm{~m}), 2936(\mathrm{~m}), 1727$ (s), $1458(\mathrm{~m}), 1368(\mathrm{~s}), 1255(\mathrm{~m}), 1154$ (s), $1035(\mathrm{~m})$

MS: $\quad(\mathrm{EI}, 70 \mathrm{eV})$ $242\left(\mathrm{M}^{+}, 1\right), 225(1), 186(20), 169(18), 113$ (100), 74 (11), 57 (46)

Opt. Rot.: $\quad+14.34(\mathrm{c}=1.0, \mathrm{EtOH})$

TLC: $\quad R_{f} 0.30$ (hexanes/EtOAc, 9/1) $\left[\mathrm{UV}(254) / \mathrm{KMnO}_{4}\right]$

SFC: $\quad t_{R}(2 S, 3 R)-9 \mathrm{el}, 2.21$ (94.3\%); $t_{R}(2 R, 3 S)$-9el, 2.54 (5.7\%) (Chiralpak AS, 125 psi, $40{ }^{\circ} \mathrm{C}, 1.5 \% \mathrm{CH}_{3} \mathrm{OH}$ in $\mathrm{CO}_{2}, 3.5 \mathrm{~mL} / \mathrm{min}, 220 \mathrm{~nm}$ )

Analysis: $\quad \mathrm{C}_{12} \mathrm{H}_{18} \mathrm{O}_{3} \mathrm{~S}(242.34)$

Calcd: $\quad$ C, $59.47 ; \quad$ H, $7.49 \%$

Found; C, 59.72; H, $7.68 \%$ 
Preparation of tert-Butyl 3-Hydroxy-2-methyl-5-phenyl-4-pentenoate (9eg)

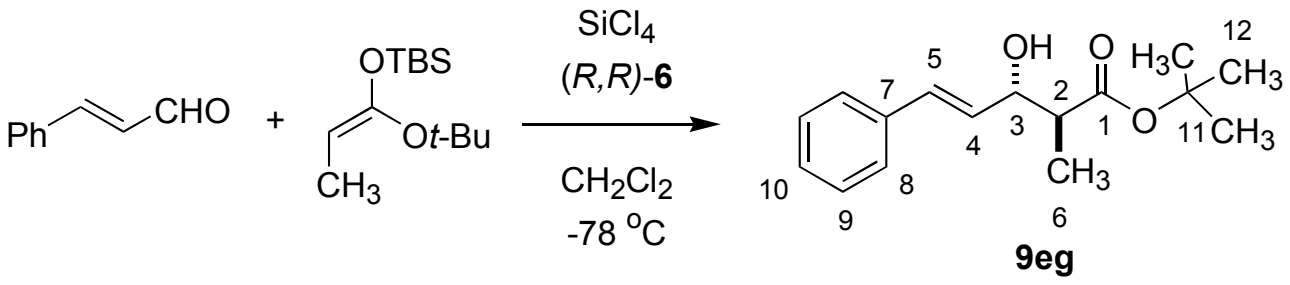

Following General Procedure 5, $141 \mu \mathrm{L}(1.0 \mathrm{mmol})$ of cinnamaldehyde was combined with 8.4 $\mathrm{mg}(0.01 \mathrm{mmol}, 0.01$ equiv) of bis-phosphoramide $((R, R)-6), 123 \mu \mathrm{L}(1.1 \mathrm{mmol}, 1.1$ equiv) of $\mathrm{SiCl}_{4}$, and $293 \mathrm{mg}$ (1.2 mmol, 1.2 equiv) of (E)-1-[(tert-butoxy)-propenyl]-tert-butyldimethylsilane (2e) to yield, after Kugelrohr distillation, $256 \mathrm{mg}$ (98\%) of tert-butyl 3-hydroxy2-methyl-5-phenyl-pent-4-enoate (9eg) as a white crystalline solid.

Data for 9eg:

mp: $\quad 80-82^{\circ} \mathrm{C}$

bp: $\quad 200{ }^{\circ} \mathrm{C}(0.01 \mathrm{mmHg}, \mathrm{ABT})$

${ }^{1} \mathrm{H}$ NMR: $\quad\left(399 \mathrm{MHz}, \mathrm{CDCl}_{3}\right)$

7.15 (m, $5 \mathrm{H}, \mathrm{HC}(8,9,10)), 6.64(\mathrm{~d}, J=15.9,1 \mathrm{H}, \mathrm{HC}(5)), 6.19$ (dd, $J=15.9,6.6$, $1 \mathrm{H}, \mathrm{HC}(4)), 4.33$ (t, $J=6.6,1 \mathrm{H}, \mathrm{HC}(3)), 2.55$ (pent, $J=7.1,1 \mathrm{H}, \mathrm{HC}(2)$ ), 1.46 (s, $\left.9 \mathrm{H}, \mathrm{H}_{3} \mathrm{C}(12)\right), 1.20\left(\mathrm{~d}, J=7.3,3 \mathrm{H}, \mathrm{H}_{3} \mathrm{C}(6)\right)$

${ }^{13} \mathrm{C} \mathrm{NMR}: \quad\left(100 \mathrm{MHz}, \mathrm{CDCl}_{3}\right)$

174.97 (C(1)), 136.50 (C(7)), 131.73 (C(5)), 129.53 (C(4)), 128.55 (C(8)), 127.73 (C(10)), 126.50 (C(9)), 81.24 (C(11)), 74.62 (C(3)), 46.19 (C(2)), 28.05 (C(12)), $14.25(\mathrm{C}(6))$

IR: (neat) $3521(\mathrm{w}), 3011$ (m), 2982 (m), 2937 (m), 1718 (s), 1457 (w), 1369 (m), 1154 (s), $968(\mathrm{~m})$

MS.: $\quad(\mathrm{EI}, 70 \mathrm{eV})$ $263\left(\mathrm{M}^{+1}, 3\right), 245$ (20), 206 (37), 189 (99), 143 (25), 133 9100), 105 (16), 57 (86)

TLC: $\quad R_{f} 0.36$ (hexanes/EtOAc, $\left.8 / 2\right)\left[\mathrm{UV}(254) / \mathrm{KMnO}_{4}\right]$

Opt. Rot.: $\quad[\alpha]_{\mathrm{D}}^{24}-17.48(\mathrm{c}=1, \mathrm{EtOH})$

SFC: $\quad t_{R}(2 S, 3 S)-9 \mathrm{eg}, 3.31(>99.0 \%) ; t_{R}(2 R, 3 R)-9 \mathrm{eg}, 4.13 \mathrm{~min}(<1.0 \%)$ (Chiralpak AS, $150 \mathrm{psi}, 40^{\circ} \mathrm{C}, 1.0 \% \mathrm{CH}_{3} \mathrm{OH}$ in $\mathrm{CO}_{2}, 3.5 \mathrm{~mL} / \mathrm{min}, 220 \mathrm{~nm}$ ) 
Analysis: $\quad \mathrm{C}_{16} \mathrm{H}_{22} \mathrm{O}_{3}(262.34)$

Calcd: $\quad$ C, $73.25 ; \quad H, 8.45 \%$

Found: $\quad$ C, $73.18 ; \quad H, 8.45 \%$

Preparation of tert-Butyl 3-Hydroxy-2,4-dimethyl-5-phenyl-4-pentenoate (9eh)

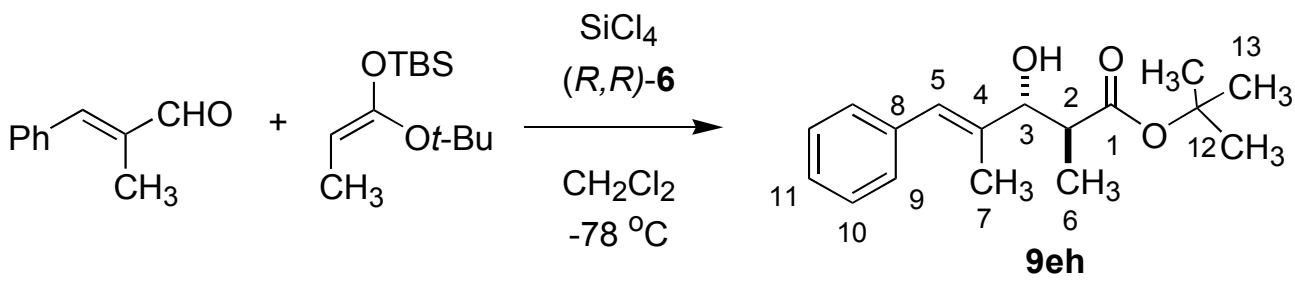

Following General Procedure 5, $140 \mu \mathrm{L}(1.0 \mathrm{mmol})$ of $\alpha$-methylcinnamaldehyde was combined with $8.4 \mathrm{mg}$ (0.01 mmol, 0.01 equiv) of bis-phosphoramide $((R, R)-6), 123 \mu \mathrm{L}(1.1 \mathrm{mmol}, 1.1$ equiv) of $\mathrm{SiCl}_{4}$, and $293 \mathrm{mg}$ (1.2 mmol, 1.2 equiv) of (E)-1-[(tert-butoxy)-propenyl]-tert-butyldimethylsilane (2e) to yield, after Kugelrohr distillation, $248 \mathrm{mg}$ (90\%) of tert-butyl 3-hydroxy2,4-dimethyl-5-phenyl-pent-4-enoate (9eh) as a colorless wax.

Data for 9eh:

bp: $\quad 225^{\circ} \mathrm{C}(0.01 \mathrm{mmHg}, \mathrm{ABT})$

${ }^{1} \mathrm{H}$ NMR: $\quad\left(399 \mathrm{MHz}, \mathrm{CDCl}_{3}\right)$

7.25 (m, 5 H, HC(9,10,11)), 6.53 (s, $1 \mathrm{H}, \mathrm{HC}(5)), 4.20$ (d, J= 7.8, $1 \mathrm{H}, \mathrm{HC}(3))$, 3.00 (s, $1 \mathrm{H}, \mathrm{OH}), 2.67$ (pent, $J=7.8,1 \mathrm{H}, \mathrm{HC}(2)), 1.87$ (s, $\left.3 \mathrm{H}, \mathrm{H}_{3} \mathrm{C}(7)\right), 1.47$ (s, $\left.9 \mathrm{H}, \mathrm{H}_{3} \mathrm{C}(13)\right), 1.13\left(\mathrm{~d}, J=7.3,3 \mathrm{H}, \mathrm{H}_{3} \mathrm{C}(6)\right)$

${ }^{13} \mathrm{C} \mathrm{NMR}: \quad\left(100 \mathrm{MHz}, \mathrm{CDCl}_{3}\right)$

$175.28(\mathrm{C}(1)), 137.33(\mathrm{C}(4)), 137.24(\mathrm{C}(8)), 128.98$ (C(10)), 128.07 (C(11)), 127.83 (C(9)), 126.54 (C(5)), 81.22 (C(12)), 79.88 (C(3)), 43.92 (C(2)), 28.04 (C(12)), 14.67 (C(7)), $13.10(\mathrm{C}(6))$

IR: $\quad\left(\mathrm{CHCl}_{3}\right)$ 3454 (w), 2977 (s), 2935 (m), 1726 (s), 1454 (m), 1368 (m), 1254 (m), 1154 (s), $1009(\mathrm{~m}), 649(\mathrm{~m})$

MS: $\quad(\mathrm{EI}, 70 \mathrm{eV})$ $277\left(\mathrm{M}^{+1}, 2\right), 261$ (2), 220 (19), 203 (43), 157 (43), 157 (24), 147 (100), 129 (27), $91(18), 57(38)$ 
TLC: $\quad R_{f} 0.39$ (hexanes/EtOAc, $\left.8 / 2\right)\left[\mathrm{UV}(254) / \mathrm{KMnO}_{4}\right]$

Opt. Rot.: $\quad[\alpha]_{D}^{24}-66.28(\mathrm{c}=1.0, \mathrm{EtOH})$

SFC: $\quad t_{R}(2 S, 3 R)$-9eh, $3.63(96.1 \%) ; t_{R}(2 R, 3 S)$-9eh, $4.13 \mathrm{~min}$ (3.9\%) (Chiralpak AS, 150 psi, $40{ }^{\circ} \mathrm{C}, 1.0 \% \mathrm{CH}_{3} \mathrm{OH}$ in $\mathrm{CO}_{2}, 3.0 \mathrm{~mL} / \mathrm{min}, 220 \mathrm{~nm}$ )

Analysis: $\quad \mathrm{C}_{17} \mathrm{H}_{24} \mathrm{O}_{3}(276.38)$

$\begin{array}{lll}\text { Calcd: } & \text { C, } 73.88 ; & \text { H, } 8.75 \% \\ \text { Found: } & \text { C, } 73.59 ; & \text { H, } 8.63 \%\end{array}$

\section{Preparation of tert-Butyl 3-Hydroxy-2-methyl-4-hexenoate (9em)}

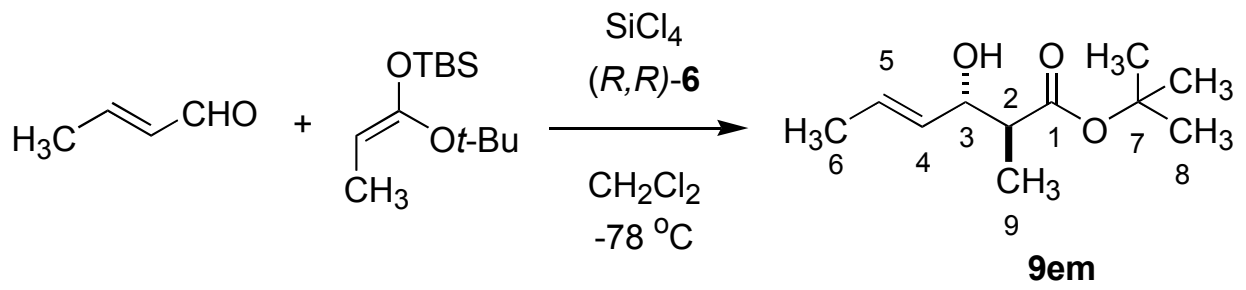

A flame-dried, $25 \mathrm{~mL}, 2$-neck flask containing a solution of $8.4 \mathrm{mg}$ of bis-phosphoramide $(R, R)$ 6 (0.01 mmol, 0.01 equiv) and $82 \mu \mathrm{L}$ of crotonaldehyde $5 \mathrm{~m}(1.0 \mathrm{mmol})$ in $\mathrm{CH}_{2} \mathrm{Cl}_{2}(5 \mathrm{~mL})$ was cooled to $-78{ }^{\circ} \mathrm{C}$ under nitrogen. To the resulting solution was added $123 \mu \mathrm{L}$ of $\mathrm{SiCl}_{4}(1.1 \mathrm{mmol}$, 1.1 equiv). Then $293 \mathrm{mg}$ of $2 \mathrm{e}$ ( $1.2 \mathrm{mmol}, 1.2$ equiv) was added dropwise over $5 \mathrm{~min}$ to the reaction mixture. The solution was allowed to stir at $-78{ }^{\circ} \mathrm{C}$ (bath temperature) for $3 \mathrm{~h}$ whereupon a solution $464 \mu \mathrm{L}$ of triethylamine ( $3.3 \mathrm{mmol}, 3.3$ equiv) in $\mathrm{MeOH}(2 \mathrm{~mL}$ ) was added by cannula. The resulting solution was allowed to warm to room temperature and stirred for $1 \mathrm{~h}$ prior to pouring into a rapidly stirring, sat. sodium bicarbonate solution $(20 \mathrm{~mL})$. This mixture was stirred vigorously for $1 \mathrm{~h}$ before filtration through Celite. The aqueous layer was washed with $\mathrm{CH}_{2} \mathrm{Cl}_{2}(3 \times 25 \mathrm{~mL})$. The combined organic extracts were washed with brine $(50 \mathrm{~mL})$, dried over $\mathrm{Na}_{2} \mathrm{SO}_{4}(2 \mathrm{~g})$, filtered and the filtrate was concentrated in vacuo. The residue was purifed by silica gel column chromatography (hexanes/EtOAc, 9/1) to yield $109 \mathrm{mg}(55 \%)$ of $9 \mathbf{e m}$ as a colorless oil. Enantiomeric composition was determined on the benzoate derivative $\mathbf{3 2}$. 


\section{Data for 9em:}

${ }^{1} \mathrm{H}$ NMR: $\quad\left(400 \mathrm{MHz}, \mathrm{CDCl}_{3}\right)$

$5.72(\mathrm{ddq}, J=15.4,0.9,6.6,1 \mathrm{H}, \mathrm{HC}(5)), 5.45$ (ddq, $J=15.4,7.4,1.7,1 \mathrm{H}$, $\mathrm{HC}(4)), 4.08$ (t, $J=7.1,1 \mathrm{H}, \mathrm{HC}(3)), 2.7$ (bs, $1 \mathrm{H}, \mathrm{OH}$ ), 2.41 (pent, $J=7.1,1 \mathrm{H}$, $\mathrm{HC}(2)), 1.70$ (dd, $\left.J=6.6,1.2,3 \mathrm{H}, \mathrm{H}_{3} \mathrm{C}(6)\right), 1.45$ (s, $\left.9 \mathrm{H}, \mathrm{H}_{3} \mathrm{C}(8)\right), 1.11$ (d, $J=$ 7.3, $\left.3 \mathrm{H}, \mathrm{H}_{3} \mathrm{C}(9)\right)$

${ }^{13} \mathrm{C} \mathrm{NMR}: \quad\left(100 \mathrm{MHz}, \mathrm{CDCl}_{3}\right)$

175.13 (C(1)), 131.29 (C(5)), 128.54 (C(4)), 80.98 (C(7)), 74.73 (C(3)), 46.19

(C(2)), $28.07(\mathrm{C}(8)), 17.71(\mathrm{C}(6)), 14.13(\mathrm{C}(9))$

IR: (neat)

3448 (m), 2977 (s), 2937 (m), 2883 (w), 1727 (s), 1457 (m), 1369 (s), 1225 (m), $1159(\mathrm{~s}), 1031(\mathrm{~m})$

MS: $\quad(\mathrm{EI}, 70 \mathrm{eV})$

$201\left(\mathrm{M}^{+}, 7\right), 183$ (5), 145 (22), 127 (100), 109 (5), 71 (12)

Opt. Rot.: $\quad[\alpha]_{\mathrm{D}}^{24}-17.17(\mathrm{c}=1.0, \mathrm{EtOH})$

TLC: $\quad R_{f} 0.25$ (hexanes/EtOAc, 9/1) $\left[\mathrm{KMnO}_{4}\right]$

SFC: $\quad t_{R}(2 S, 3 S)-32,6.57(98.2 \%) ; t_{R}(2 R, 3 R)-32,7.50(1.8 \%)$ (Chiralpak AD, 125 psi, $40{ }^{\circ} \mathrm{C}, 1.0 \% \mathrm{CH}_{3} \mathrm{OH}$ in $\mathrm{CO}_{2}, 2.0 \mathrm{~mL} / \mathrm{min}, 220 \mathrm{~nm}$ )

Analysis: $\quad \mathrm{C}_{11} \mathrm{H}_{20} \mathrm{O}_{3}(200.14)$
Calcd:
C, 65.97;
H, $10.07 \%$

Found;

C, 65.79;

$\mathrm{H}, 10.18 \%$ 


\section{Preparation of tert-Butyl 3-Benzoyloxy-2-methyl-4-hexenoate (32)}

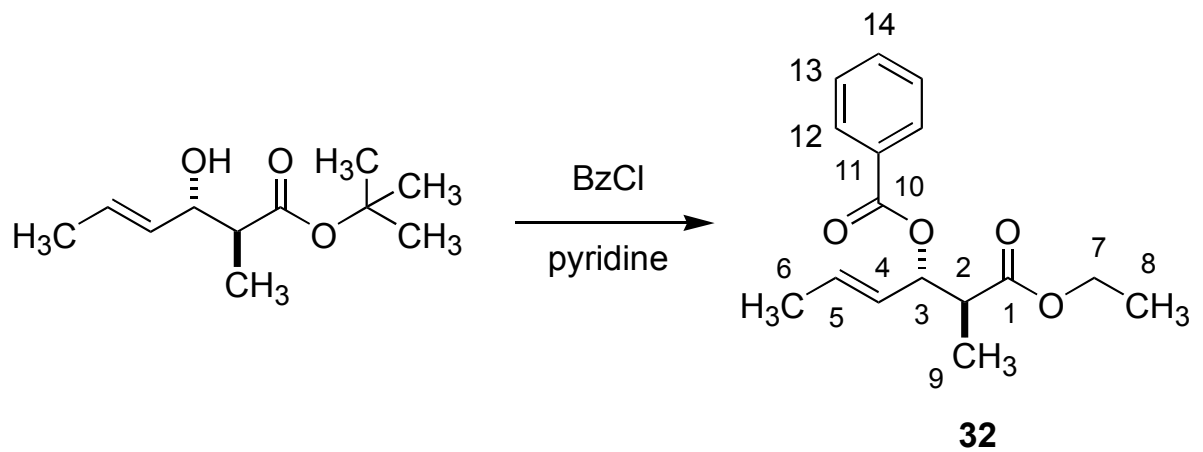

To a stirred solution of $39 \mathrm{mg}$ of tert-butyl 3-hydroxy-2-methyl-4-hexenoate 9em (0.2 mmol) in pyridine $(1.0 \mathrm{~mL})$ and $1 \mathrm{~mL}$ of THF was added $30 \mu \mathrm{L}$ of benzoyl chloride $(0.2 \mathrm{mmol}, 1$ equiv) at $0{ }^{\circ} \mathrm{C}$ under nitrogen. After the addition was completed, the solution was allowed to stir at room temperature for $24 \mathrm{~h}$. The reaction mixture was then diluted with $\mathrm{Et}_{2} \mathrm{O}(10 \mathrm{~mL})$, washed with 0.1 $\mathrm{M} \mathrm{HCl}(20 \mathrm{~mL})$, sat. $\mathrm{NaHCO}_{3}(20 \mathrm{~mL})$ and brine $(20 \mathrm{~mL})$. The organic layer was dried over $\mathrm{Na}_{2} \mathrm{SO}_{4}(2 \mathrm{~g})$ and filtered. The filtrate was concentrated in vacuo. Purification of the residue by silica gel column chromatography (hexanes/EtOAc, 9/1), afforded $40 \mathrm{mg}$ (67\%) of 32 as a colorless oil.

\section{Data for 32:}

${ }^{1} \mathrm{H} \mathrm{NMR}: \quad\left(400 \mathrm{MHz}, \mathrm{CDCl}_{3}\right)$

8.03 (m, 2 H, HC(12)), 7.53 (m, 1 H, HC(14)), 7.41 (m, 2 H, HC(13)), 5.92 (ddq, $J=15.2,0.7,6.6,1 \mathrm{H}, \mathrm{HC}(5)), 5.56(\mathrm{t}, J=8.4,1 \mathrm{H}, \mathrm{HC}(3)), 5.43(\mathrm{ddq}, J=8.1$, 15.2, 1.7, $1 \mathrm{H}, \mathrm{HC}(4)), 2.76$ (pent, $J=7.1,1 \mathrm{H}, \mathrm{HC}(2)), 1.71$ (dd, $J=6.4,1.3,3$ $\left.\mathrm{H}, \mathrm{H}_{3} \mathrm{C}(6)\right), 1.35$ (s, $\left.9 \mathrm{H}, \mathrm{H}_{3} \mathrm{C}(8)\right), 1.15$ (d, $J=7.1,3 \mathrm{H}, \mathrm{H}_{3} \mathrm{C}(9)$ )

${ }^{13} \mathrm{C} \mathrm{NMR}: \quad\left(100 \mathrm{MHz}, \mathrm{CDCl}_{3}\right)$ 172.29 (C(1)), 165.29 (C(10)), 132.84 (C(14)), 132.06 (C(5)), 130.42 (C(10)), 129.60 (C(12)), 128.27 (C(13)), 126.64 (C(4)), 80.59 (C(7)), 76.74 (C(3)), 45.26 (C(2)), 27.88 (C(8)), 17.78 (C(6)), 13.19 (C(9))

TLC: $\quad R_{f} 0.44$ (hexanes/EtOAc, 9/1) [UV(254)/KMnO

SFC: $\quad t_{R}(2 S, 3 S)-32,6.57(98.2 \%) ; t_{R}(2 R, 3 R)-32,7.50(1.8 \%)$ (Chiralpak AD, 125 psi, $40{ }^{\circ} \mathrm{C}, 1.0 \% \mathrm{CH}_{3} \mathrm{OH}$ in $\mathrm{CO}_{2}, 2.0 \mathrm{~mL} / \mathrm{min}, 220 \mathrm{~nm}$ ) 
Preparation of tert-Butyl 3-Hydroxy-2-ethyl-5-phenyl-4-pentynoate (9en)

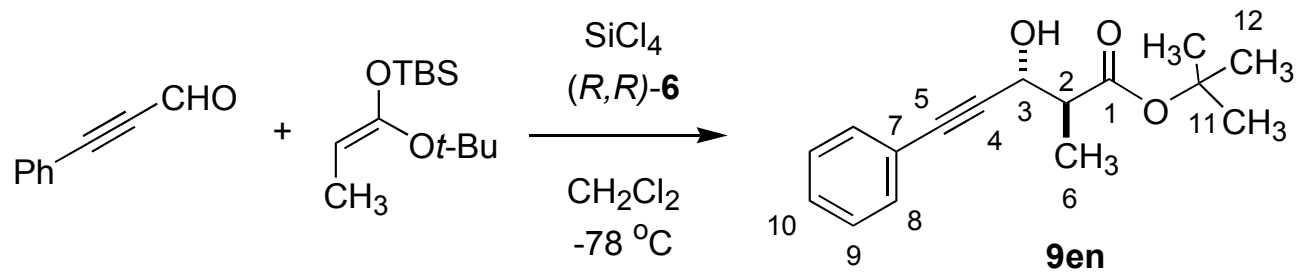

Following General Procedure 5, $122 \mu \mathrm{L}(1.0 \mathrm{mmol})$ of 3-phenyl-2-propynal was combined with $8.4 \mathrm{mg}(0.01 \mathrm{mmol}, 0.01$ equiv) of bis-phosphoramide $((R, R)-6), 123 \mu \mathrm{L}(1.1 \mathrm{mmol}, 1.1$ equiv) of $\mathrm{SiCl}_{4}$, and $293 \mathrm{mg}$ (1.2 mmol, 1.2 equiv) of $(E)$-1-[(tert-butoxy)-propenyl]-tert-butyldimethylsilane (2e) to yield, after Kugelrohr distillation, $240 \mathrm{mg}$ (92\%) of tert-butyl 3-hydroxy2-methyl-5-phenyl-pent-4-ynoate $((2 S, 3 R)-9 e n)$ as a white crystalline solid.

Data for 9en:

bp: $\quad 210^{\circ} \mathrm{C}(0.01 \mathrm{mmHg}, \mathrm{ABT})$

mp: $\quad 68-70{ }^{\circ} \mathrm{C}$

${ }^{1} \mathrm{H}$ NMR: $\quad\left(499 \mathrm{MHz}, \mathrm{CDCl}_{3}\right)$

7.42 (m, 2 H, HC(8)), 7.30 (m, 3 H, HC(9,10)), 4.67 (t, $J=7.1,1$ H, HC(3)), 3.09

(d, $J=7.1,1 \mathrm{H}, \mathrm{OH}), 2.73$ (pent, $J=7.1,1 \mathrm{H}, \mathrm{HC}(2)), 1.48$ (s, $9 \mathrm{H}, \mathrm{H}_{3} \mathrm{C}(12)$ ), $1.32\left(\mathrm{~d}, J=7.3,3 \mathrm{H}, \mathrm{H}_{3} \mathrm{C}(6)\right)$

${ }^{13} \mathrm{C} \mathrm{NMR}: \quad\left(125 \mathrm{MHz}, \mathrm{CDCl}_{3}\right)$

174.10 (C(1)), 131.71 (C(9)), 128.48 (C(10)), 128.27 (C(8)), 122.45 (C(7)), 87.96 $(\mathrm{C}(5)), 85.54(\mathrm{C}(4)), 81.46$ (C(11)), 64.85 (C(3)), 46.95 (C(2)), 28.02 (C(12)), $13.95(\mathrm{C}(6))$

IR: $\quad\left(\mathrm{CHCl}_{3}\right)$ 3485 (w), 3028 (w), 3010 (m), 2982 (m), 2938 (m), 1712 (s), 1600 (w), 1490 (w), $1458(\mathrm{w}), 1369$ (m), 1253 (m), 1155 (s), $1028(\mathrm{w})$

MS: $\quad(\mathrm{EI}, 70 \mathrm{eV})$

$261\left(\mathrm{M}^{+}, 23\right), 243$ (8), 205 (44), 187 (100), 131 (39)

TLC: $\quad R_{f} 0.41$ (hexanes/EtOAc, 8/2) $\left[\mathrm{UV}(254) / \mathrm{KMnO}_{4}\right]$

Opt. Rot.: $\quad[\alpha]_{\mathrm{D}}^{24}+6.87(\mathrm{c}=1.0, \mathrm{EtOH})$

SFC: $\quad t_{R}(2 S, 3 R)-9 \mathrm{en}, 4.78(84.5 \%) ; t_{R}(2 R, 3 S)-9 \mathrm{en}, 5.71 \mathrm{~min}(15.5 \%)$ (Chiralpak AS, $150 \mathrm{psi}, 40{ }^{\circ} \mathrm{C}, 1.5 \% \mathrm{CH}_{3} \mathrm{OH}$ in $\mathrm{CO}_{2}, 2.5 \mathrm{~mL} / \mathrm{min}, 220 \mathrm{~nm}$ ) 
Analysis: $\quad \mathrm{C}_{16} \mathrm{H}_{20} \mathrm{O}_{3}(260.33)$

Calcd: $\quad$ C, $73.82 ; \quad \mathrm{H}, 7.74 \%$

Found: $\quad$ C, $73.92 ; \quad H, 7.76 \%$

Preparation of Ethyl 3-Hydroxy-2-methyl-5-phenylpentanoate (9bk)

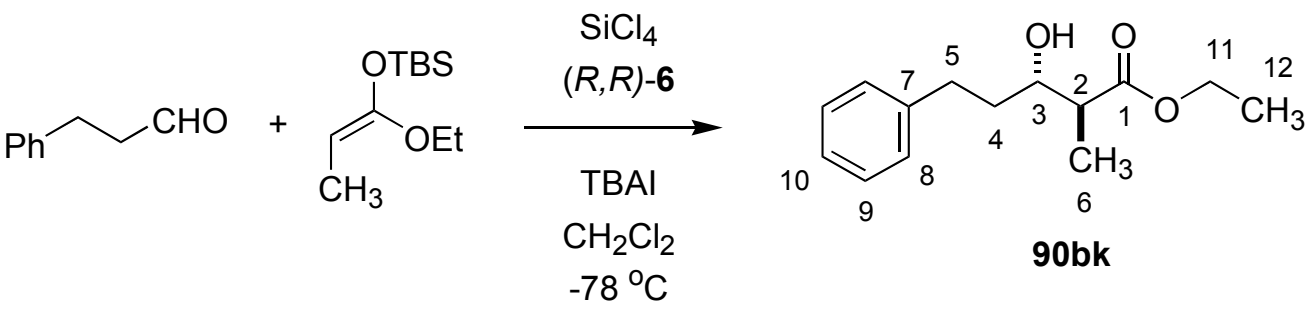

A flame-dried, 10-mL, 2-neck round bottom flask containing a solution of $42.1 \mathrm{mg}(0.05 \mathrm{mmol}$, 0.05 equiv) of bis-phosphoramide $((R, R)-6)$ and $37 \mathrm{mg}(0.1 \mathrm{mmol}, 0.1$ equiv) of tetrabutylammonium iodide in $\mathrm{CH}_{2} \mathrm{Cl}_{2}(2.5 \mathrm{~mL})$ was cooled to $-78{ }^{\circ} \mathrm{C}$ under nitrogen. Into this mixture was added $123 \mu \mathrm{L}\left(1.1 \mathrm{mmol}, 1.1\right.$ equiv) of $\mathrm{SiCl}_{4}$. To the resulting mixture was added $132 \mu \mathrm{L}(1.0 \mathrm{mmol})$ of hydrocinnamaldehyde and it was allowed to stir at $-78{ }^{\circ} \mathrm{C}$ for $5 \mathrm{~min}$. Then $260 \mathrm{mg}$ (1.2 mmol, 1.2 equiv) of (E)-[(1-ethoxy)-propenyl]-tert-butyl-dimethylsilane (2b) was added dropwise (over $5 \mathrm{~min}$ ) to the reaction mixture. The resulting mixture was allowed to stir at $-78{ }^{\circ} \mathrm{C}$ (bath temperature) for $24 \mathrm{~h}$ whereupon the cold reaction mixture was poured into a rapidly stirring $1 / 1$ sat. aq. $\mathrm{KF} / 1.0 \mathrm{M} \mathrm{KH}_{2} \mathrm{PO}_{4}$ solution $(20 \mathrm{~mL})$. This biphasic mixture was stirred vigorously for $1 \mathrm{~h}$ before filtration through Celite. The aqueous layer was washed with $\mathrm{CH}_{2} \mathrm{Cl}_{2}(3 \times 50 \mathrm{~mL})$. The combined organic extracts were washed with brine $(50 \mathrm{~mL})$, dried over $\mathrm{Na}_{2} \mathrm{SO}_{4}(2 \mathrm{~g})$, filtered and the filtrate was concentrated in vacuo. The residue was purified by silica gel column chromatography (hexanes/EtOAc, 9/1), to yield $167 \mathrm{mg}$ (71\%) of ethyl 3hydroxy-2-methyl-5-phenyl-propanoate (9bk) as a colorless oil.

\section{Data for 9bk:}

${ }^{1} \mathrm{H}$ NMR: $\quad\left(399 \mathrm{MHz}, \mathrm{CDCl}_{3}\right.$ )

7.20 (m, $5 \mathrm{H}, \mathrm{HC}(8,9,10)), 4.18$ (q, $J=7.08,2 \mathrm{H}, \mathrm{HC}(11)), 3.68$ (m, $1 \mathrm{H}, \mathrm{HC}(3))$,

2.89 (m, $1 \mathrm{H}, \mathrm{HC}(5)), 2.77$ (d, $J=7.08,1 \mathrm{H}, \mathrm{OH}), 2.67$ (m, $1 \mathrm{H}, \mathrm{HC}(3)), 2.53$

(pent, $J=6.34,1 \mathrm{H}, \mathrm{HC}(2)), 1.80(\mathrm{~m}, 2 \mathrm{H}, \mathrm{HC}(4)), 1.28(\mathrm{t}, J=7.08,3 \mathrm{H}$, $\left.\mathrm{H}_{3} \mathrm{C}(12)\right), 1.22\left(\mathrm{~d}, J=7.32,3 \mathrm{H}, \mathrm{H}_{3} \mathrm{C}(6)\right)$ 
${ }^{13} \mathrm{C}$ NMR: $\quad\left(100 \mathrm{MHz}, \mathrm{CDCl}_{3}\right)$

$176.1(\mathrm{C}(1)), 141.94(\mathrm{C}(9)), 128.46(\mathrm{C}(10)), 128.39$ (C(8)), 125.85 (C(7)), 72.63

(C(11)), $60.64(\mathrm{C}(3)), 45.15(\mathrm{C}(2)), 36.67(\mathrm{C}(5)), 31.91(\mathrm{C}(4)), 14.41(\mathrm{C}(12))$, $14.17(\mathrm{C}(6))$

IR: (neat)

3459 (m), 3026 (w), 2980 (s), 2940 (s), 1726 (s), 1495 (w), 1455 (s), 1376 (s), $1262(\mathrm{~s}), 1187(\mathrm{~s}), 1096(\mathrm{~s}), 1043(\mathrm{~s})$

SFC: $\quad t_{R}(2 S, 3 S)-9 \mathbf{b k}, 4.34(94.9 \%) ; t_{R}(2 R, 3 R)-9 \mathbf{b k}, 5.04 \mathrm{~min}(5.2 \%)$ (Chiralpak OD, $150 \mathrm{psi}, 40{ }^{\circ} \mathrm{C}, 2.0 \% \mathrm{CH}_{3} \mathrm{OH}$ in $\mathrm{CO}_{2}, 3.5 \mathrm{~mL} / \mathrm{min}, 220 \mathrm{~nm}$ )

MS: $\quad(E I, 70 \mathrm{eV})$

$237\left(\mathrm{M}^{+}, 53\right), 219$ (52), 173 (19), 144 (65), 117 (100), 91 (73)

TLC: $\quad R_{f} 0.1$ (hexanes/EtOAc, 9/1) $\left[\mathrm{UV}(254) / \mathrm{KMnO}_{4}\right]$

Opt. Rot.: $\quad[\alpha]_{\mathrm{D}}^{24}-14.87(\mathrm{c}=1.0, \mathrm{EtOH})$

Analysis: $\quad \mathrm{C}_{14} \mathrm{H}_{20} \mathrm{O}_{3}(236.31)$

Calcd: $\quad$ C, $71.16 ; \quad \mathrm{H}, 8.53 \%$

Found: $\quad$ C, $70.95 ; \quad H, 8.52 \%$

\section{Preparation of Ethyl 3-Cyclohexyl-3-hydroxy-2-methylpropanoate (9bj)}

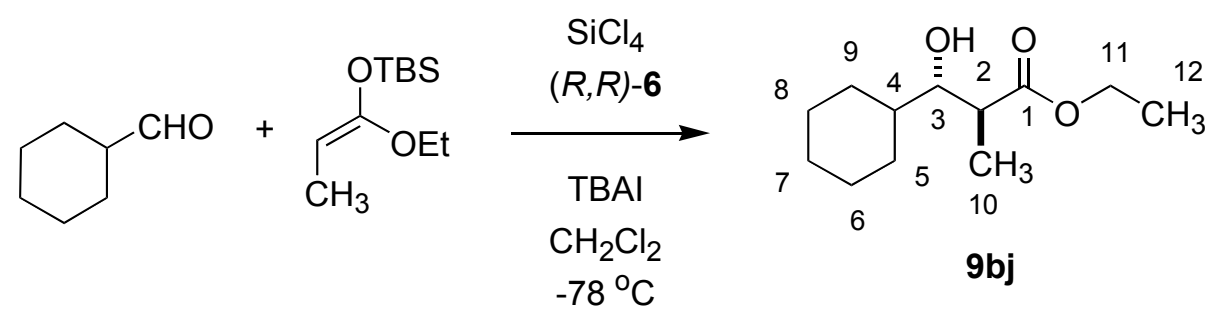

A flame-dried, 10-mL, 2-neck round bottom flask containing a solution of $84.2 \mathrm{mg}(0.1 \mathrm{mmol}$, 0.1 equiv) of bis-phosphoramide $((R, R)-6)$ and $37 \mathrm{mg}(0.1 \mathrm{mmol}, 0.1$ equiv) of tetrabutylammonium iodide in $\mathrm{CH}_{2} \mathrm{Cl}_{2}(1.25 \mathrm{~mL})$ was cooled to $-40{ }^{\circ} \mathrm{C}$ under nitrogen. Into this mixture was added $123 \mu \mathrm{L}$ ( $1.1 \mathrm{mmol}, 1.1$ equiv) of $\mathrm{SiCl}_{4}$. To the resulting mixture was added $121 \mu \mathrm{L}(1.0 \mathrm{mmol})$ of cyclohexanecarboxaldehyde and it was allowed to stir at $-78^{\circ} \mathrm{C}$ for $5 \mathrm{~min}$. Then $260 \mathrm{mg}$ (1.2 mmol, 1.2 equiv) of (E)-[(1-ethoxy)-propenyl]-tert-butyl-dimethylsilane (2b) was added dropwise (over $5 \mathrm{~min}$ ) to the reaction mixture. The resulting mixture was allowed to 
stir at $-40{ }^{\circ} \mathrm{C}$ (bath temperature) for $24 \mathrm{~h}$ whereupon the cold reaction mixture was poured into a rapidly stirring $1 / 1$ sat. aq. $\mathrm{KF} / 1.0 \mathrm{M} \mathrm{KH}_{2} \mathrm{PO}_{4}$ solution $(20 \mathrm{~mL})$. This biphasic mixture was stirred vigorously for $1 \mathrm{~h}$ before filtration through Celite. The aqueous layer was washed with $\mathrm{CH}_{2} \mathrm{Cl}_{2}(3 \times 50 \mathrm{~mL})$. The combined organic extracts were washed with brine $(50 \mathrm{~mL})$, dried over $\mathrm{Na}_{2} \mathrm{SO}_{4}(2 \mathrm{~g})$, filtered and the filtrate was concentrated in vacuo. The residue was purified by silica gel column chromatography (hexanes/EtOAc, 9/1), to yield $86 \mathrm{mg}(40 \%)$ of ethyl 3cyclohexyl-3-hydroxy-2-methyl propanoate (9bj) as a colorless oil. Enantiomeric composition was determined on the 3,5-dinitrobenzoate derivative 33 .

Data for $9 \mathbf{b j}$ :

${ }^{1} \mathrm{H}$ NMR: $\quad\left(399 \mathrm{MHz}, \mathrm{CDCl}_{3}\right)$

4.17 (m, $2 \mathrm{H}, \mathrm{HC}(11)$ ), 3.35 (q, $J=6.6,1 \mathrm{H}, \mathrm{HC}(3)$ ), 2.65 (pent, $J=5.9,1 \mathrm{H}$, $\mathrm{HC}(2)), 2.57$ (d, $J=7.8,1 \mathrm{H}, \mathrm{OH}), 1.85-1.65$ (m, $4 \mathrm{H}, \mathrm{HC}(6,8)), 1.6-1.5(\mathrm{~m}, 4 \mathrm{H}$, $\mathrm{HC}(5,9)), 1.27$ (t, $\left.J=7.1,3 \mathrm{H}, \mathrm{H}_{3} \mathrm{C}(12)\right), 1.22$ (d, $\left.J=7.08,3 \mathrm{H}, \mathrm{H}_{3} \mathrm{C}(10)\right), 1.2-1.0$ $(\mathrm{m}, 3 \mathrm{H}, \mathrm{HC}(4,7))$

${ }^{13} \mathrm{C} \mathrm{NMR}: \quad\left(100 \mathrm{MHz}, \mathrm{CDCl}_{3}\right)$

$176.57(\mathrm{C}(1)), 77.79(\mathrm{C}(11)), 60.52(\mathrm{C}(3)), 41.79(\mathrm{C}(2)), 41.19(\mathrm{C}(4)), 29.91$ $(\mathrm{C}(7)), 27.13(\mathrm{C}(5)), 26.36(\mathrm{C}(6)), 26.32(\mathrm{C}(8)), 26.04(\mathrm{C}(9)), 14.91(\mathrm{C}(12))$, $14.19(\mathrm{C}(10))$

IR: (neat)

3498 (w), $2979(\mathrm{w}), 2853$ (s), 1721 (s), $1450(\mathrm{~m}), 1376(\mathrm{~m}), 1258(\mathrm{~m}), 1179(\mathrm{~s})$, $1102(\mathrm{~m}), 1036(\mathrm{~m})$

SFC: $\quad t_{R}(2 S, 3 S)-\mathbf{3 3}, 8.30(67.8 \%) ; t_{R}(2 R, 3 R)-\mathbf{3 3}, 9.16 \min$ (32.8\%) (Chiralpak OD, 150 psi, $40{ }^{\circ} \mathrm{C}, 3.0 \% \mathrm{CH}_{3} \mathrm{OH}$ in $\mathrm{CO}_{2}, 3.0 \mathrm{~mL} / \mathrm{min}, 220 \mathrm{~nm}$ )

MS: $\quad(E I, 70 \mathrm{eV})$

215 ( $\left.\mathrm{M}^{+1}, 17\right), 197$ (37), 169 (9), 151 (28), 131 (100), 123 (26), 102 (83), 85 (64), 74 (51), 57 (34)

TLC: $\quad R_{f} 0.23$ (hexanes/EtOAc, 9/1) $\left[\mathrm{UV}(254) / \mathrm{KMnO}_{4}\right]$

Opt. Rot.: $\quad[\alpha]_{\mathrm{D}}^{24}+5.26(\mathrm{c}=1.0, \mathrm{EtOH})$

Analysis: $\quad \mathrm{C}_{12} \mathrm{H}_{22} \mathrm{O}_{3}(214.16)$

Calcd: $\quad$ C, $67.26 ; \quad$ H, $10.35 \%$

Found: $\quad$ C, $67.03 ; \quad H, 10.55 \%$ 


\section{Preparation of Ethyl 3-Cyclohexyl-3-(3,5-dinitrobenzoyloxy)-2-methylpropanoate (33)}

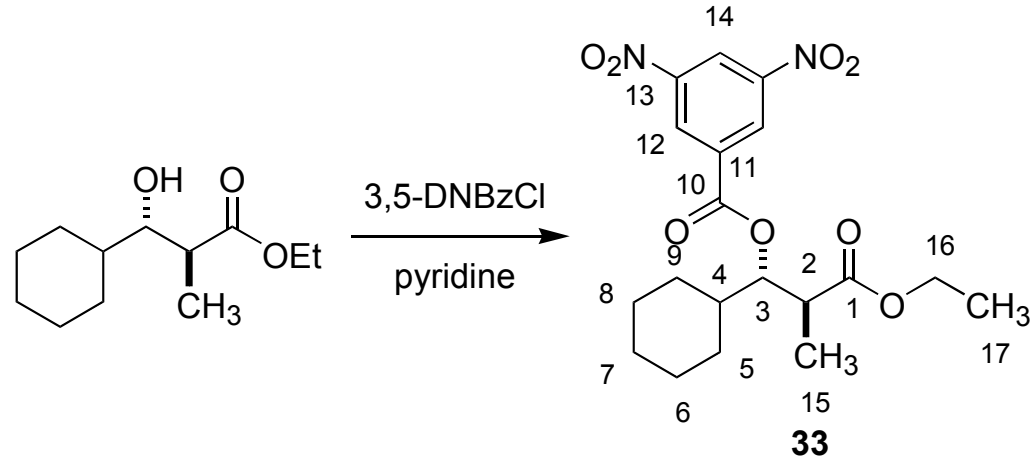

To a stirred solution of $134 \mathrm{mg}(0.627 \mathrm{mmol})$ of $(2 S, 3 S)-9 \mathbf{b j}$ in pyridine $(1.0 \mathrm{~mL})$ and $1 \mathrm{~mL}$ of THF was added $145 \mathrm{mg}\left(0.627 \mathrm{mmol}, 1.0\right.$ equiv) of 3,5-dinitrobenzoyl chloride at $0{ }^{\circ} \mathrm{C}$ under nitrogen. After the addition was completed, the mixture was stirred at room temperature for $5 \mathrm{~h}$. The reaction mixture was then diluted with $\mathrm{Et}_{2} \mathrm{O}(10 \mathrm{~mL})$, washed with $0.1 \mathrm{M} \mathrm{HCl}(20 \mathrm{~mL})$, sat. $\mathrm{NaHCO}_{3}(20 \mathrm{~mL})$ and brine $(20 \mathrm{~mL})$. The organic layer was dried over $\mathrm{Na}_{2} \mathrm{SO}_{4}(2 \mathrm{~g})$ and filtered. The filtrate was concentrated in vacuo. Purification of the residue by silica gel chromatography (hexane/EtOAc, 9/1), afforded $220 \mathrm{mg}$ (86\%) of ethyl 3-cyclohexyl-3-(3,5dinitrobenzoyloxy)-2-methyl propanoate (33).

Data for 33:

1ㄱ NMR: $\quad\left(499 \mathrm{MHz}, \mathrm{CDCl}_{3}\right)$

9.22 (S, $1 \mathrm{H}, \mathrm{HC}(13)), 9.14$ (S, $2 \mathrm{H}, \mathrm{HC}(12)), 5.29$ (t, $J=6.10 \mathrm{HC}(3)), 4.12$ (q, $J$

$=7.08,2 \mathrm{H} \mathrm{HC}(15)), 3.01$ (pent, $J=6.38,1 \mathrm{H}, \mathrm{HC}(2))$, 1.83-1.65 (m, $8 \mathrm{H}$, $\mathrm{HC}(5,6,8,9)), 1.27$ (t, $\left.J=7.08,3 \mathrm{H}, \mathrm{H}_{3} \mathrm{C}(16)\right), 1.22$ (d, $\left.J=7.0,3 \mathrm{H}, \mathrm{H}_{3} \mathrm{C}(14)\right)$, 1.2-0.9 (m, $2 \mathrm{H}, \mathrm{HC}(4,7))$

${ }^{13} \mathrm{C} \mathrm{NMR}: \quad\left(125 \mathrm{MHz}, \mathrm{CDCl}_{3}\right)$

173.60 (C(1)), 162.32 (C(10)), 148.94 (C(13)), 134.21 (C(11)), 129.76 (C(12)), 122.64 (C(14)), 81.64 (C(16)), 61.11 (C(3)), 41.53 (C(2)), 39.39 (C(4)), 30.15 (C(7)), 27.44 (C(5)), 26.30 (C(6)), 26.22 (C(8)), 26.02 (C(9)), 14.43 (C(17)), $14.33(\mathrm{C}(15))$

SFC: $\quad t_{R}(2 S, 3 S)-33,8.30(67.8 \%) ; t_{R}(2 R, 3 R)-33,9.16$ min (32.8\%) (Chiralpak OD, 150 psi, $40{ }^{\circ} \mathrm{C}, 3.0 \% \mathrm{CH}_{3} \mathrm{OH}$ in $\left.\mathrm{CO}_{2}, 3.0 \mathrm{~mL} / \mathrm{min}, 220 \mathrm{~nm}\right)$

TLC: $\quad R_{f} 0.25$ (hexanes/EtOAc, 9/1) [UV(254)] 


\section{Preparation of Ethyl 3-Hydroxy-2,5-dimethylhexanoate (9bo)}

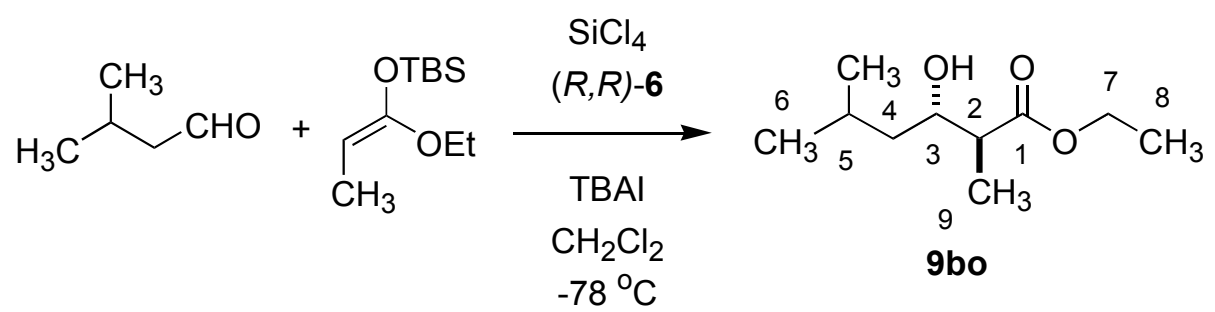

A flame-dried, $25 \mathrm{~mL}, 2$-neck flask containing a solution of $42 \mathrm{mg}$ of bis-phosphoramide $(R, R)-6$ (0.05 mmol, 0.05 equiv), $8 \mu \mathrm{L}$ of $i-\operatorname{Pr}_{2} \operatorname{EtN}(0.05 \mathrm{mmol}, 0.05$ equiv) and $107 \mu \mathrm{L}$ of isovaleraldehyde $50(1.0 \mathrm{mmol})$ in $\mathrm{CH}_{2} \mathrm{Cl}_{2}(5 \mathrm{~mL})$ was cooled to $-78{ }^{\circ} \mathrm{C}$ under nitrogen. To the resulting solution was added $123 \mu \mathrm{L}$ of $\mathrm{SiCl}_{4}(1.1 \mathrm{mmol}, 1.1$ equiv). Then $260 \mathrm{mg}$ of $\mathbf{2 b}(1.2$ mmol, 1.2 equiv) was added dropwise over $5 \mathrm{~min}$ to the reaction mixture. The solution was allowed to stir at $-78{ }^{\circ} \mathrm{C}$ (bath temperature) for $24 \mathrm{~h}$ whereupon the cold reaction mixture was poured into a rapidly stirring $1 / 1$ sat. aq. $\mathrm{KF} / 1.0 \mathrm{M} \mathrm{KH}_{2} \mathrm{PO}_{4}$ solution $(20 \mathrm{~mL})$. This biphasic mixture was stirred vigorously for $1 \mathrm{~h}$ before filtration through Celite. The aqueous layer was washed with $\mathrm{CH}_{2} \mathrm{Cl}_{2}(3 \times 25 \mathrm{~mL})$. The combined organic extracts were washed with brine (50 $\mathrm{mL})$, dried over $\mathrm{Na}_{2} \mathrm{SO}_{4}(2 \mathrm{~g})$, filtered and the filtrate was concentrated in vacuo. The residue was purifed by silica gel column chromatography (hexanes/EtOAc, 9/1) to yield $142 \mathrm{mg}(76 \%)$ of $\mathbf{9 b o}$ as a colorless oil. Enantiomeric analysis was determined on the benzoate derivative 34 . Data for 9bo:

${ }^{1} \mathrm{H}$ NMR: $\quad\left(400 \mathrm{MHz}, \mathrm{CDCl}_{3}\right)$

$4.17\left(\mathrm{~m}, 2 \mathrm{H}, \mathrm{H}_{2} \mathrm{C}(7)\right), 3.72(\mathrm{ddd}, J=9.6,6.0,3.0,1 \mathrm{H}, \mathrm{HC}(3)), 2.46$ (pent, $J=$ 6.9, $1 \mathrm{H}, \mathrm{HC}(2)), 1.85$ (m, $1 \mathrm{H}, \mathrm{HC}(5)), 1.39$ (ddd, $J=14.1,10.1,4.5,1 \mathrm{H}$, $\mathrm{HC}(4)), 1.27$ (t, $\left.J=7.1,3 \mathrm{H}, \mathrm{H}_{3} \mathrm{C}(8)\right), 1.25$ (m, $\left.1 \mathrm{H}, \mathrm{HC}(4)\right), 1.21$ (d, $J=7.1,3 \mathrm{H}$, $\left.\mathrm{H}_{3} \mathrm{C}(9)\right), 0.93$ (d, $\left.J=7.1,3 \mathrm{H}, \mathrm{H}_{3} \mathrm{C}(6)\right), 0.91$ (d, $\left.J=7.1,3 \mathrm{H}, \mathrm{H}_{3} \mathrm{C}(6)\right)$

${ }^{13} \mathrm{C} \mathrm{NMR}: \quad\left(100 \mathrm{MHz}, \mathrm{CDCl}_{3}\right)$

$176.14(\mathrm{C}(1)), 71.49$ (C(3)), 60.51 (C(7)), 45.64 (C(2)), 44.03 (C(5)), 24.45

(C(4)), 23.63 (C(8)), 21.62 (C(9)), 14.33 (C(6)), 14.17 (C(6))

IR: (neat)

3475 (m), 2956 (s), 2871 (m), 1722 (s), 1467 (m), 1371 (m), 1257 (m), 1186 (s), 1130 (m), 1034 (m) 
MS: $\quad(\mathrm{EI}, 70 \mathrm{eV})$

$189\left(\mathrm{M}^{+1}, 8\right), 171(7), 143(6), 131$ (61), 102 (100), 85 (31), 74 (52), 57 (15)

Opt. Rot.: $\quad[\alpha]_{\mathrm{D}}^{24}-12.90(\mathrm{c}=1.0, \mathrm{EtOH})$

TLC: $\quad R_{f} 0.29$ (hexanes/EtOAc, 9/1) $\left[\mathrm{KMnO}_{4}\right]$

SFC: $\quad t_{R}(2 S, 3 S)-34,4.62(89.7 \%) ; t_{R}(2 R, 3 R)-34,5.09$ (10.3\%) (Chiralpak OD, 125 psi, $40{ }^{\circ} \mathrm{C}, 1.0 \% \mathrm{CH}_{3} \mathrm{OH}$ in $\mathrm{CO}_{2}, 2.0 \mathrm{~mL} / \mathrm{min}, 220 \mathrm{~nm}$ )

Analysis: $\quad \mathrm{C}_{10} \mathrm{H}_{20} \mathrm{O}_{3}(188.14)$

Calcd: $\quad$ C, 63.80; $\quad$ H, $10.71 \%$

Found; $\quad$ C, 63.60; H, 10.88\%

\section{Preparation of Ethyl 3-Benzoyloxy-2,5-dimethylhexanoate (34)}

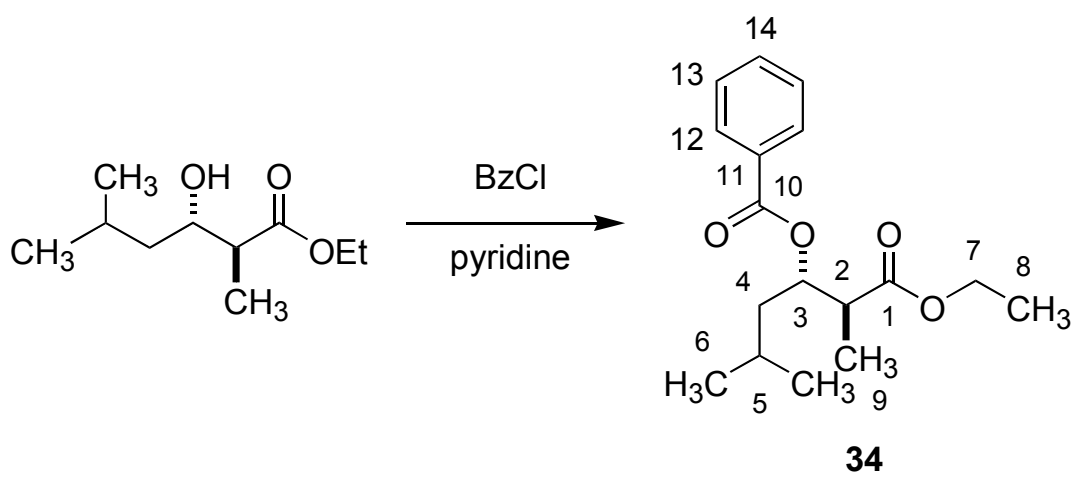

To a stirred solution of $51 \mathrm{mg}$ of ethyl 3-hydroxy-2-methyl-4-hexanoate 9 bo $(0.31 \mathrm{mmol})$ in pyridine $(1.0 \mathrm{~mL})$ and $1 \mathrm{~mL}$ of THF was added $37 \mu \mathrm{L}$ of benzoyl chloride $(0.31 \mathrm{mmol}, 1$ equiv) at $0{ }^{\circ} \mathrm{C}$ under nitrogen. After the addition was completed, the solution was allowed to stir at room temperature for $24 \mathrm{~h}$. The reaction mixture was then diluted with $\mathrm{Et}_{2} \mathrm{O}(10 \mathrm{~mL})$, washed with 0.1 $\mathrm{M} \mathrm{HCl}(20 \mathrm{~mL})$, sat. $\mathrm{NaHCO}_{3}(20 \mathrm{~mL})$ and brine $(20 \mathrm{~mL})$. The organic layer was dried over $\mathrm{Na}_{2} \mathrm{SO}_{4}(2 \mathrm{~g})$ and filtered. The filtrate was concentrated in vacuo. Purification of the residue by silica gel column chromatography (hexanes/EtOAc, 9/1), afforded $52 \mathrm{mg}$ (62\%) of 34 as a colorless oil.

Data for 34:

${ }^{1} \mathrm{H}$ NMR: $\quad\left(400 \mathrm{MHz}, \mathrm{CDCl}_{3}\right)$

8.05-7.26 (m, 5 H, HC(12,13,14)), 5.47 (ddd, $J=9.2,6.0,3.2,1$ H, HC(3)), 4.12

(m, $\left.2 \mathrm{H}, \mathrm{H}_{2} \mathrm{C}(7)\right), 2.91$ (pent, $\left.J=5.8,1 \mathrm{H}, \mathrm{HC}(2)\right), 1.74$ (m, $\left.2 \mathrm{H}, \mathrm{HC}(4,5)\right), 1.39$ 
(m, $1 \mathrm{H}, \mathrm{HC}(4)), 1.20$ (t, $\left.J=7.1,3 \mathrm{H}, \mathrm{H}_{3} \mathrm{C}(8)\right), 1.19$ (d, $\left.J=7.1,3 \mathrm{H}, \mathrm{H}_{3} \mathrm{C}(9)\right)$,

$0.95\left(\mathrm{~d}, J=6.4,3 \mathrm{H}, \mathrm{H}_{3} \mathrm{C}(6)\right), 0.93\left(\mathrm{~d}, J=6.6,3 \mathrm{H}, \mathrm{H}_{3} \mathrm{C}(6)\right)$

${ }^{13} \mathrm{C} \mathrm{NMR}: \quad\left(100 \mathrm{MHz}, \mathrm{CDCl}_{3}\right)$

173.40 (C(1)), 165.92 (C(10)), 137.94 (C(14)), 130.30 (C(11)), 129.65 (C(12)), 128.36 (C(13)), 73.53 (C(7)), 60.58 (C(3)), 43.71 (C(2)), 40.21 (C(5)), 24.56

(C(8)), 23.51 (C(4)), 21.82 (C(9)), 14.14 (C(6)), 12.25 (C(6))

TLC: $\quad R_{f} 0.52$ (hexanes/EtOAc, 9/1) [UV(254)/ $\left.\mathrm{KMnO}_{4}\right]$

SFC: $\quad t_{R}(2 S, 3 S)-344.62(89.7 \%) ; t_{R}(2 R, 3 R)-34,5.09(10.3 \%)$ (Chiralpak OD, 125 psi, $40{ }^{\circ} \mathrm{C}, 1.0 \% \mathrm{CH}_{3} \mathrm{OH}$ in $\mathrm{CO}_{2}, 2.0 \mathrm{~mL} / \mathrm{min}, 220 \mathrm{~nm}$ )

\section{Preparation of Ethyl 3-Hydroxy-2-methylbenzenepropanoate (11aa)}

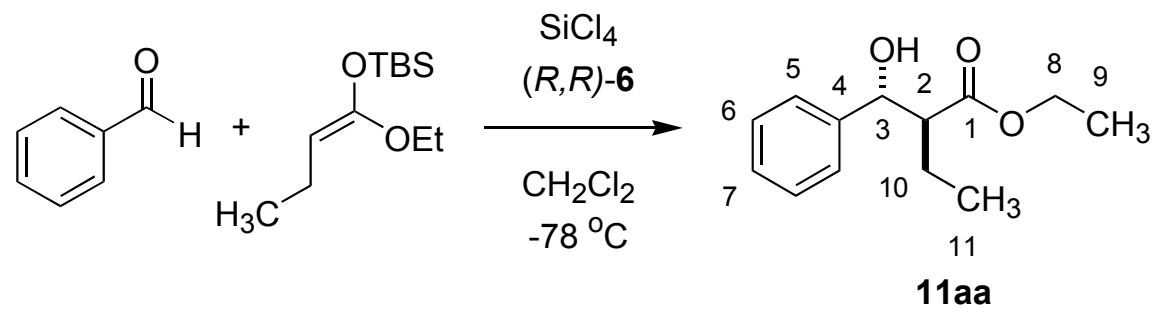

Following General Procedure 5, $8.4 \mathrm{mg}$ of bis-phosphoramide $(R, R)-6$ (0.01 mmol, 0.01 equiv) was combined with $102 \mu \mathrm{L}$ of benzaldehyde 5a $(1.0 \mathrm{mmol}), 123 \mu \mathrm{L}$ of $\mathrm{SiCl}_{4}(1.1 \mathrm{mmol}, 1.1$ equiv) and $276 \mathrm{mg}$ of 10a (1.2 mmol, 1.2 equiv) to yield, after silica gel column chromatography (hexanes/EtOAc, 9/1), $167 \mathrm{mg}$ (75\%) of 11 aa as a colorless oil.

\section{Data for 11aa:}

${ }^{1} \mathrm{H}$ NMR: $\quad\left(400 \mathrm{MHz}, \mathrm{CDCl}_{3}\right)$

$7.32(\mathrm{~m}, 5 \mathrm{H}, \mathrm{HC}(5,6,7)), 4.80(\mathrm{~d}, J=7.6,1 \mathrm{H}, \mathrm{HC}(3)), 4.16\left(\mathrm{~m}, 2 \mathrm{H}, \mathrm{H}_{2} \mathrm{C}(10)\right)$,

2.87 (s, $1 \mathrm{H}, \mathrm{OH}), 2.67$ (m, $1 \mathrm{H}, \mathrm{HC}(2)), 1.58$ (m, $1 \mathrm{H}, \mathrm{HC}(10)), 1.39$ (m, $1 \mathrm{H}$, $\mathrm{HC}(10)), 1.22$ (t, $\left.J=7.1,3 \mathrm{H}, \mathrm{H}_{3} \mathrm{C}(9)\right), 0.87$ (t, $\left.J=6.6,3 \mathrm{H}, \mathrm{H}_{3} \mathrm{C}(11)\right)$

${ }^{13} \mathrm{C} \mathrm{NMR}: \quad\left(100 \mathrm{MHz}, \mathrm{CDCl}_{3}\right)$

175.26 (C(1)), 142.07 (C(4)), 128.46 (C(6)), 127.92 (C(7)), 126.39 (C(5)), 75.08 (C(3)), 60.57 (C(8)), 54.62 (C(2)), 22.27 (C(10)), 14.21 (C(9)), 11.55 (C(11))

IR: (neat) 3596 (w), 2969 (m), 1725 (s), 1456 (m), 1378 (m), 1186 (s), 1027 (m) 
MS: $\quad(\mathrm{EI}, 70 \mathrm{eV})$

$222\left(\mathrm{M}^{+}, 2\right), 205$ (4), $193(3), 177$ (2), $116(100), 107$ (61), 77 (74)

Opt. Rot.: $\quad[\alpha]_{\mathrm{D}}^{24}+23.31(\mathrm{c}=1.0, \mathrm{EtOH})$

TLC: $\quad R_{f} 0.16$ (hexanes/EtOAc, 9/1) [UV(254)/ $\left.\mathrm{KMnO}_{4}\right]$

SFC: $\quad t_{R}(2 S, 3 R)-11 \mathbf{a a}, 2.07(83.6 \%) ; t_{R}(2 R, 3 S)-11 \mathbf{a}, 2.58$ (16.4\%) (Chiralpak AS, 150 psi, $40{ }^{\circ} \mathrm{C}, 3.0 \% \mathrm{CH}_{3} \mathrm{OH}$ in $\mathrm{CO}_{2}, 4.0 \mathrm{~mL} / \mathrm{min}, 220 \mathrm{~nm}$ )

Analysis: $\quad \mathrm{C}_{13} \mathrm{H}_{18} \mathrm{O}_{3}(222.13)$

Calcd: $\quad$ C, $70.24 ; \quad H, 8.16 \%$

Found; $\quad$ C, $70.12 ; \quad$ H, $8.09 \%$

\section{Preparation of Ethyl 3-Hydroxy-2-(1-methylethyl)benzenepropanoate (11ba)}

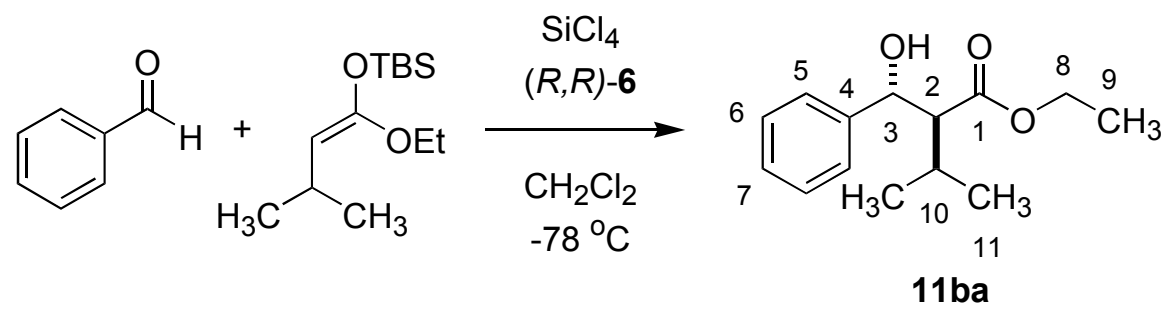

Following General Procedure 5, $8.4 \mathrm{mg}$ of bis-phosphoramide $(R, R)-6$ (0.01 mmol, 0.01 equiv) was combined with $102 \mu \mathrm{L}$ of benzaldehyde $5 \mathbf{a}(1.0 \mathrm{mmol}), 123 \mu \mathrm{L}$ of $\mathrm{SiCl}_{4}(1.1 \mathrm{mmol}, 1.1$ equiv) and $293 \mathrm{mg}$ of $\mathbf{1 0 b}$ (1.2 mmol, 1.2 equiv) to yield, after silica gel column chromatography (hexanes/EtOAc, 9/1), $193 \mathrm{mg}$ (82\%) of $\mathbf{1 1 b a}$ as a colorless oil.

\section{Data for 11ba:}

${ }^{1} \mathrm{H}$ NMR: $\quad\left(400 \mathrm{MHz}, \mathrm{CDCl}_{3}\right)$

$7.31(\mathrm{~m}, 5 \mathrm{H}, \mathrm{HC}(5,6,7)), 4.96$ (dd, $J=7.8,5.6,1 \mathrm{H}, \mathrm{HC}(3)), 4.03$ (q, $J=7.3,2 \mathrm{H}$,

$\left.\mathrm{H}_{2} \mathrm{C}(8)\right), 3.41(\mathrm{~d}, J=8.3,1 \mathrm{H}, \mathrm{OH}), 2.52(\mathrm{dd}, J=8.1,5.6,1 \mathrm{H}, \mathrm{HC}(2)), 2.02$

(octet, $J=6.6,1 \mathrm{H}, \mathrm{HC}(10)), 1.07$ (t, $\left.J=7.1,3 \mathrm{H}, \mathrm{H}_{3} \mathrm{C}(9)\right), 1.06(\mathrm{~d}, J=6.8,3 \mathrm{H}$, $\left.\mathrm{H}_{3} \mathrm{C}(11)\right), 0.94$ (d, $\left.J=6.8,3 \mathrm{H}, \mathrm{H}_{3} \mathrm{C}(11)\right)$

${ }^{13} \mathrm{C} \mathrm{NMR:} \quad\left(100 \mathrm{MHz}, \mathrm{CDCl}_{3}\right)$

174.98 (C(1)), 142.47 (C(4)), 128.32 (C(6)), 127.47 (C(7)), 125.74 (C(5)), 72.47

(C(3)), 60.32 (C(8)), 59.28 (C(2)), 27.86 (C(9)), 20.98 (C(10)), 20.00 (C(11)), $14.07(\mathrm{C}(11))$ 
IR: (neat)

3488 (m), 2964 (s), 2935 (m), 2824 (m), 1730 (s), 1465 (m), 1454 (m), 1390 (m), 1375 (s), 1287 (s), 1234 (s), 1179 (s), 1160 (s), 1060 (m), 1028 (m)

MS: $\quad(\mathrm{EI}, 70 \mathrm{eV})$

$236\left(\mathrm{M}^{+}, 3\right), 219(6), 193$ (6), 173 (4), 145 (6), 130 (75), 115 (100), 87 (37)

Opt. Rot.: $\quad[\alpha]_{\mathrm{D}}^{24}+19.88(\mathrm{c}=1.0, \mathrm{EtOH})$

TLC: $\quad R_{f} 0.25$ (hexanes/EtOAc, 9/1) [UV(254)/ $\left.\mathrm{KMnO}_{4}\right]$

SFC: $\quad t_{R}(2 R, 3 S)-11 b a, 2.23(14.2 \%) ; t_{R}(2 S, 3 R)-11$ ba, $3.83(85.8 \%)$ (Chiralpak OD, 125 psi, $40{ }^{\circ} \mathrm{C}, 1.5 \% \mathrm{CH}_{3} \mathrm{OH}$ in $\mathrm{CO}_{2}, 3.5 \mathrm{~mL} / \mathrm{min}, 220 \mathrm{~nm}$ )

Analysis: $\quad \mathrm{C}_{14} \mathrm{H}_{20} \mathrm{O}_{3}(236.31)$

Calcd: $\quad$ C, $71.16 ; \quad \mathrm{H}, 8.53 \%$

Found; $\quad$ C, $71.05 ; \quad H, 8.54 \%$

Preparation of Ethyl 3-Hydroxy-2-(2-methylpropyl)benzenepropanoate (11ca)

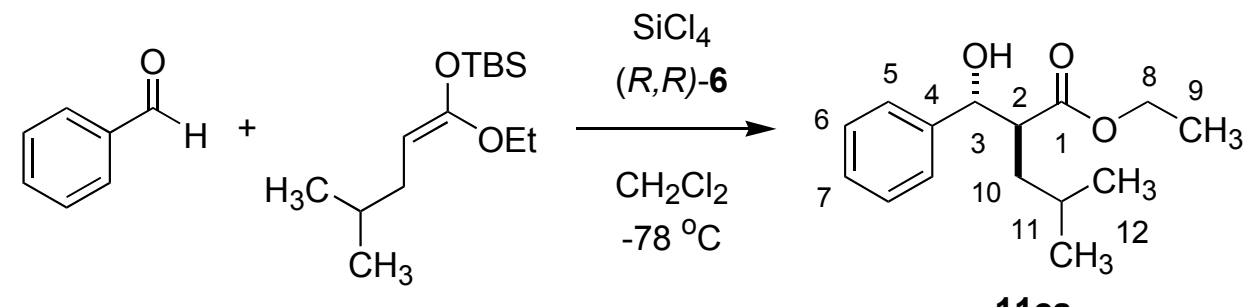

11ca

Following General Procedure 5, $8.4 \mathrm{mg}$ of bis-phosphoramide $(R, R)-6$ (0.01 mmol, 0.01 equiv) was combined with $102 \mu \mathrm{L}$ of benzaldehyde $\mathbf{5 a}(1.0 \mathrm{mmol}), 123 \mu \mathrm{L}$ of $\mathrm{SiCl}_{4}(1.1 \mathrm{mmol}, 1.1$ equiv) and $310 \mathrm{mg}$ of 10c (1.2 mmol, 1.2 equiv) to yield, after silica gel column chromatography (hexanes/EtOAc, 9/1), $219 \mathrm{mg}$ (88\%) of 11ca as a colorless oil.

\section{Data for 11ca:}

${ }^{1} \mathrm{H}$ NMR: $\quad\left(400 \mathrm{MHz}, \mathrm{CDCl}_{3}\right)$

$7.36(\mathrm{~m}, 5 \mathrm{H}, \mathrm{HC}(5,6,7)), 4.75$ (t, $J=6.6,1 \mathrm{H}, \mathrm{HC}(3)), 4.13\left(\mathrm{~m}, 2 \mathrm{H}, \mathrm{H}_{2} \mathrm{C}(8)\right)$,

$2.87(\mathrm{~d}, J=5.6,1 \mathrm{H}, \mathrm{OH}), 2.84(\mathrm{ddd}, J=10.7,7.3,4.6,1 \mathrm{H}, \mathrm{HC}(2)), 1.65$ (ddd, $J$

$=13.7,10.7,5.1,1 \mathrm{H}, \mathrm{HC}(10)), 1.51(\mathrm{~m}, 1 \mathrm{H}, \mathrm{HC}(11)), 1.21(\mathrm{t}, J=7.1,3 \mathrm{H}$,

$\left.\mathrm{H}_{3} \mathrm{C}(9)\right), 1.08$ (ddd, $\left.J=13.7,9.3,4.6,1 \mathrm{H}, \mathrm{HC}(10)\right), 0.84$ (d, $J=2.7,3 \mathrm{H}$,

$\left.\mathrm{H}_{3} \mathrm{C}(12)\right), 0.82\left(\mathrm{~d}, J=2.5,3 \mathrm{H}, \mathrm{H}_{3} \mathrm{C}(12)\right)$ 
${ }^{13} \mathrm{C}$ NMR: $\quad\left(100 \mathrm{MHz}, \mathrm{CDCl}_{3}\right)$

175.55 (C(1)), $142.17(\mathrm{C}(4)), 128.47$ (C(6)), 127.90 (C(7)), 126.32 (C(5)), 75.78

(C(3)), $60.54(\mathrm{C}(8)), 51.13(\mathrm{C}(2)), 38.69(\mathrm{C}(10)), 26.06(\mathrm{C}(11)), 23.21(\mathrm{C}(12))$, $21.53(\mathrm{C}(12)), 14.15(\mathrm{C}(9))$

IR: (neat)

3596 (m), 3498 (w), 2960 (s), 2872 (m), 1726 (s), 1469 (m), 1454 (m), 1378 (m), $1246(\mathrm{~m}), 1181(\mathrm{~s}), 1017(\mathrm{~m})$

MS: $\quad(E I, 70 \mathrm{eV})$

$250\left(\mathrm{M}^{+}, 2\right), 233$ (2), 205 (1), 159 (3), 144 (38), 101 (100), 73 (34)

Opt. Rot.: $\quad[\alpha]_{\mathrm{D}}^{24}-2.51(\mathrm{c}=1.0, \mathrm{EtOH})$

TLC: $\quad R_{f} 0.25$ (hexanes/EtOAc, 9/1) $\left[\mathrm{UV}(254) / \mathrm{KMnO}_{4}\right]$

SFC: $\quad t_{R}(2 S, 3 R)-11 \mathrm{ca}, 4.59$ (86.3\%); $t_{R}(2 R, 3 S)$-11 ca, 7.58 (13.7\%) (Chiralpak AS, 125 psi, $40{ }^{\circ} \mathrm{C}, 1.0 \% \mathrm{CH}_{3} \mathrm{OH}$ in $\left.\mathrm{CO}_{2}, 3.5 \mathrm{~mL} / \mathrm{min}, 220 \mathrm{~nm}\right)$

Analysis: $\quad \mathrm{C}_{15} \mathrm{H}_{22} \mathrm{O}_{3}(250.33)$

Calcd: $\quad$ C, $71.97 ; \quad$ H, $8.86 \%$

Found; $\quad$ C, $71.95 ; \quad \mathrm{H}, 9.06 \%$

\section{Preparation of (3S) Methyl 3-Hydroxy-2,2-dimethyl-3-phenylpropanoate ((3S)-12)}

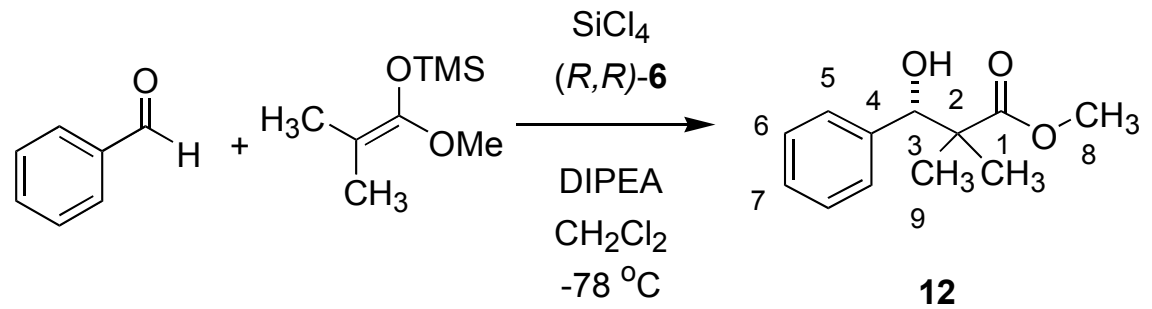

A flame-dried, $25 \mathrm{~mL}, 2$-neck, round-bottom flask containing a solution of $42 \mathrm{mg}$ of bisphosphoramide $(R, R)-6$ ( $0.05 \mathrm{mmol}, 0.05$ equiv), $8 \mu \mathrm{L}$ of $i-\operatorname{Pr}_{2} \operatorname{EtN}(0.05 \mathrm{mmol}, 0.05$ equiv) and $102 \mu \mathrm{L}$ of benzaldehyde $5 \mathrm{a}(1.0 \mathrm{mmol})$ in $\mathrm{CH}_{2} \mathrm{Cl}_{2}(5 \mathrm{~mL})$ was cooled to $-78{ }^{\circ} \mathrm{C}$ under nitrogen. To the resulting solution was added $123 \mu \mathrm{L}$ of $\mathrm{SiCl}_{4}$ (1.1 mmol, 1.1 equiv). Then $208 \mathrm{mg}$ of 3 (1.2 mmol, 1.2 equiv) was added dropwise over $5 \mathrm{~min}$ to the reaction mixture. The solution was allowed to stir at $-78{ }^{\circ} \mathrm{C}$ for $24 \mathrm{~h}$ whereupon the cold reaction mixture was poured into a rapidly stirring $1 / 1$ sat. aq. $\mathrm{KF} / 1.0 \mathrm{M} \mathrm{KH}_{2} \mathrm{PO}_{4}$ solution $(20 \mathrm{~mL})$. This biphasic mixture was stirred 
vigorously for $1 \mathrm{~h}$ before filtration through Celite. The aqueous layer was washed with $\mathrm{CH}_{2} \mathrm{Cl}_{2}(3$ x $25 \mathrm{~mL})$. The combined organic extracts were washed with brine $(50 \mathrm{~mL})$, dried over $\mathrm{Na}_{2} \mathrm{SO}_{4}(2$ $\mathrm{g}$ ), filtered and the filtrate was concentrated in vacuo. The residue was purified by Kugelrohr distillation, yielding $180 \mathrm{mg}(87 \%)$ of $(3 S)-12$ as a white, crystalline solid NMR spectral data matched that previously reported in the literature. ${ }^{22}$

Data for $(3 S)-\mathbf{1 2}$ :

mp: $\quad 62-64{ }^{\circ} \mathrm{C}$

bp: $\quad 175^{\circ} \mathrm{C}(0.1 \mathrm{~mm} \mathrm{Hg}, \mathrm{ABT})$

${ }^{1} \mathrm{H}$ NMR: $\quad\left(400 \mathrm{MHz}, \mathrm{CDCl}_{3}\right)$

7.32-7.26 (m, 5 H, HC(5,6,7)), 4.90 (s, $1 \mathrm{H}, \mathrm{HC}(3)), 3.72$ (s, $\left.3 \mathrm{H}, \mathrm{H}_{3} \mathrm{C}(8)\right), 3.05$

(s, $1 \mathrm{H}, \mathrm{OH}), 1.14$ (s, $\left.3 \mathrm{H}, \mathrm{H}_{3} \mathrm{C}(9)\right), 1.11$ (s, $\left.3 \mathrm{H}, \mathrm{H}_{3} \mathrm{C}(9)\right)$

${ }^{13} \mathrm{C} \mathrm{NMR}: \quad\left(100 \mathrm{MHz}, \mathrm{CDCl}_{3}\right)$

178.21 (C(1)), 139.89 (C(4)), 127.77 (C(6)), 127.75 (C(5)), 127.63 (C(7)), 78.69

(C(3)), 52.11 (C(8)), 47.67 (C(2)), 23.06 (C(9)), 19.0 (C(9))

Opt. Rot:: $\quad[\alpha]_{\mathrm{D}}^{24}+1.94(\mathrm{c}=2.4, \mathrm{MeOH})$, lit. $[\alpha]_{\mathrm{D}}^{24}+5.7(\mathrm{c}=2.49, \mathrm{MeOH})$ for $(3 S)-12^{22}$

TLC: $\quad R_{f} 0.36$ (hexanes/EtOAc, 4/1) [UV(254)/KMnO $\left.\mathrm{KM}_{4}\right]$

SFC: $\quad t_{R}(3 S)-12,3.69(91.1 \%) ; t_{R}(3 R)-12,4.25(8.9 \%)$ (Chiralpak OB, $125 \mathrm{psi}, 40{ }^{\circ} \mathrm{C}$, $2.0 \% \mathrm{CH}_{3} \mathrm{OH}$ in $\mathrm{CO}_{2}, 2.0 \mathrm{~mL} / \mathrm{min}, 220 \mathrm{~nm}$ )

Preparation of Isopropyl 3-Hydroxy-2-methyl-3-phenylpropanoate (9ca)

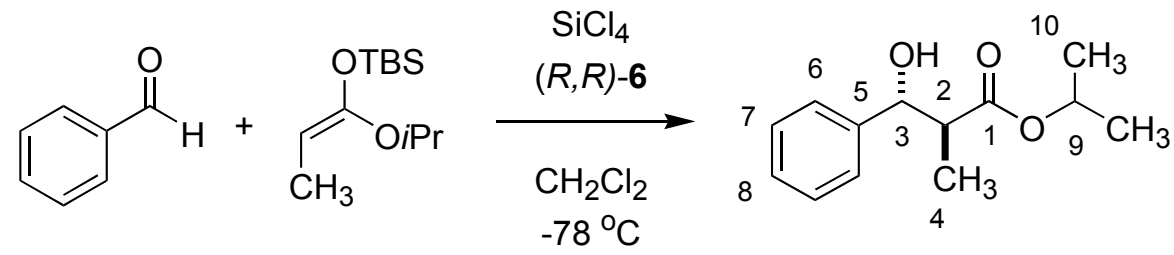

$9 \mathrm{ca}$

Following General Procedure 5, $8.4 \mathrm{mg}$ of bis-phosphoramide $(R, R)-6$ (0.01 mmol, 0.01 equiv) was combined with $102 \mu \mathrm{L}$ of benzaldehyde $5 \mathrm{a}(1.0 \mathrm{mmol}), 123 \mu \mathrm{L}$ of $\mathrm{SiCl}_{4}(1.1 \mathrm{mmol}, 1.1$ equiv) and $276 \mathrm{mg}$ of $2 \mathrm{c}$ (1.2 mmol, 1.2 equiv) to yield, after Kugelrohr distillation, $177 \mathrm{mg}$ (80 $\%$ ) of 9ca as a white crystalline solid. NMR spectral data matched that previously reported in the literature. ${ }^{9}$ 
Data for 9ca:

mp: $\quad 45-47^{\circ} \mathrm{C}$

bp: $\quad 200{ }^{\circ} \mathrm{C}(0.05 \mathrm{mmHg}, \mathrm{ABT})$

${ }^{1} \mathrm{H}$ NMR: $\quad\left(400 \mathrm{MHz}, \mathrm{CDCl}_{3}\right)$

7.36-7.26 (m, $5 \mathrm{H}, \mathrm{HC}(6,7,8)), 5.05$ (septet, $J=6.3,1 \mathrm{H}, \mathrm{HC}(9)), 4.73$ (dd, $J=$

8.3, 4.9, $1 \mathrm{H}, \mathrm{HC}(3)), 3.08(\mathrm{dd}, J=4.8,0.9,1 \mathrm{H}, \mathrm{OH}), 2.76$ (pent, $J=7.3,1 \mathrm{H}$, $\mathrm{HC}(2)), 1.23\left(\mathrm{~d}, J=6.3,3 \mathrm{H}, \mathrm{H}_{3} \mathrm{C}(10)\right), 1.20$ (d, $\left.J=6.3,3 \mathrm{H}, \mathrm{H}_{3} \mathrm{C}(10)\right), 1.02(\mathrm{~d}, J$ $\left.=7.1,3 \mathrm{H}, \mathrm{H}_{3} \mathrm{C}(4)\right)$

${ }^{13} \mathrm{C} \mathrm{NMR}: \quad\left(100 \mathrm{MHz}, \mathrm{CDCl}_{3}\right)$

175.42 (C(1)), 141.66 (C(5)), 128.42 (C(7)), 127.92 (C(8)), 126.60 (C(6)), 76.27 (C(3)), 68.15 (C(9)), 47.15 (C(2)), 21.71 (C(10)), 21.67 (C(10)), 14.54 (C(4))

IR: (neat)

3475 (m), 2980 (s), 2938 (m), 1730 (s), 1455 (m), 1376 (m), 1249 (m), 1181 (s), $1108(\mathrm{~m}), 1022(\mathrm{~m})$

MS: $\quad(\mathrm{EI}, 70 \mathrm{eV})$

$222\left(\mathrm{M}^{+}, 4\right), 179(3), 163(9), 118(5), 116(46), 107$ (74), 74 (100)

Opt. Rot.: $\quad[\alpha]_{\mathrm{D}}^{24}+31.24(\mathrm{c}=1.0, \mathrm{EtOH})$

TLC: $\quad R_{f} 0.30$ (hexanes/EtOAc, 8/2) [UV(254)/KMnO $\left.\mathrm{KM}_{4}\right]$

SFC: $\quad t_{R}(2 S, 3 R)-9 \mathbf{c a}, 4.37(91.1 \%) ; t_{R}(2 R, 3 S)-9 \mathbf{c a}, 6.38$ (8.9\%) (Chiralpak AS, 125 psi, $40{ }^{\circ} \mathrm{C}, 2.0 \% \mathrm{CH}_{3} \mathrm{OH}$ in $\mathrm{CO}_{2}, 2.0 \mathrm{~mL} / \mathrm{min}, 220 \mathrm{~nm}$ )

\section{Preparation of Ethyl 8-(tert-Butyldimethylsilyl)-3-hydroxy-2-methyloctanoate (9bp)}

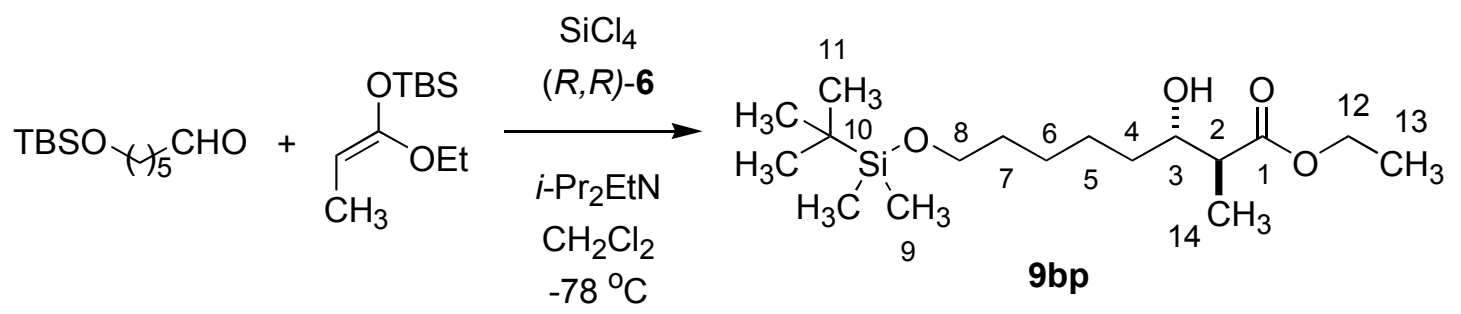

A flame-dried, $25 \mathrm{~mL}$, 2-neck, round-bottom flask containing a solution of $42 \mathrm{mg}$ of bisphosphoramide $(R, R)-6$ (0.05 mmol, 0.05 equiv), $8 \mu \mathrm{L}$ of $i-\operatorname{Pr}_{2} \operatorname{EtN}(0.05 \mathrm{mmol}, 0.05$ equiv) and $230 \mathrm{mg}$ of $\mathbf{5 p}(1.0 \mathrm{mmol})$ in $\mathrm{CH}_{2} \mathrm{Cl}_{2}(5 \mathrm{~mL})$ was cooled to $-78{ }^{\circ} \mathrm{C}$ under nitrogen. To the resulting solution was added $123 \mu \mathrm{L}$ of $\mathrm{SiCl}_{4}(1.1 \mathrm{mmol}, 1.1$ equiv). Then $260 \mathrm{mg}$ of $\mathbf{2 b}(1.2$ 
mmol, 1.2 equiv) was added dropwise over $5 \mathrm{~min}$ to the reaction mixture. The solution was allowed to stir at $-78{ }^{\circ} \mathrm{C}$ for $24 \mathrm{~h}$ whereupon the cold reaction mixture was poured into a rapidly stirring sat. aq. $\mathrm{NaHCO}_{3}$ solution $(20 \mathrm{~mL})$. This biphasic mixture was stirred vigorously for $1 \mathrm{~h}$ before filtration through Celite. The aqueous layer was washed with $\mathrm{CH}_{2} \mathrm{Cl}_{2}(3 \times 25 \mathrm{~mL})$. The combined organic extracts were washed with brine $(50 \mathrm{~mL})$, dried over $\mathrm{Na}_{2} \mathrm{SO}_{4}(2 \mathrm{~g})$, filtered and the filtrate was concentrated in vacuo. The residue was purified to yield, after silica gel column chromatography (hexanes/EtOAc, 9/1), $169 \mathrm{mg}$ (51\%) of 9bp as a colorless oil. Enantiomeric analysis was determined on the 3,5-dinitrobenzoate derivative 35 .

\section{Data for 9bp:}

${ }^{1} \mathrm{H}$ NMR: $\quad\left(400 \mathrm{MHz}, \mathrm{CDCl}_{3}\right)$

4.16 (q, $\left.J=7.1,2 \mathrm{H}, \mathrm{H}_{2} \mathrm{C}(12)\right), 3.61(\mathrm{~m}, 1 \mathrm{H}, \mathrm{HC}(3)), 3.59$ (t, $J=6.4,2 \mathrm{H}$, $\left.\mathrm{H}_{2} \mathrm{C}(8)\right), 2.59$ (d, $J=6.8,1 \mathrm{H}, \mathrm{OH}$ ), 2.49 (pent, $\left.J=6.6,1 \mathrm{H}, \mathrm{HC}(2)\right), 1.53-1.34$

(m, $8 \mathrm{H}, \mathrm{HC}(4,5,6,7)), 1.27$ (t, $\left.J=7.1,3 \mathrm{H}, \mathrm{H}_{3} \mathrm{C}(13)\right), 1.20$ (d, $J=7.3,3 \mathrm{H}$, $\left.\mathrm{H}_{3} \mathrm{C}(14)\right), 0.88$ (s, $\left.9 \mathrm{H}, \mathrm{H}_{3} \mathrm{C}(11)\right), 0.04$ (s, $6 \mathrm{H}, \mathrm{H}_{3} \mathrm{C}(9)$ )

${ }^{13} \mathrm{C} \mathrm{NMR}: \quad\left(100 \mathrm{MHz}, \mathrm{CDCl}_{3}\right)$

$176.11(\mathrm{C}(1)) 73.31(\mathrm{C}(3)), 63.16(\mathrm{C}(8)), 60.55(\mathrm{C}(12)), 45.12(\mathrm{C}(2)), 34.78$

(C(4)), $32.77(\mathrm{C}(7)), 25.96(\mathrm{C}(6)), 25.76(\mathrm{C}(11)), 25.34(\mathrm{C}(5)), 18.36(\mathrm{C}(10))$, $14.41(\mathrm{C}(13)), 14.19(\mathrm{C}(14)),-5.28(\mathrm{C}(9))$

IR: (neat) 3462 (w), 2934 (s), 2852 (s), 1735 (s), 1718 (s), 1463 (m), 1388 (m), 1255 (s), $1184(\mathrm{~s}), 1099(\mathrm{~s})$

MS: $\quad(E I, 70 \mathrm{eV})$ $333\left(\mathrm{M}^{+1}, 2\right), 288$ (3), 275 (15), 231 (14), 211 (11), 185 (13), 173 (13), 155 (12), 131 (42), 109 (100), 75 (89)

Opt. Rot.: $\quad[\alpha]_{\mathrm{D}}^{24}+5.14(\mathrm{c}=1.0, \mathrm{EtOH})$

TLC: $\quad R_{f} 0.50$ (hexanes/EtOAc, $\left.8 / 2\right)\left[\mathrm{KMnO}_{4}\right]$

SFC: $\quad t_{R}(2 R, 3 R)-35,15.78(3.3 \%) ; t_{R}(2 S, 3 S)-35,16.62$ (96.7\%) (Whelk-O1, 125 psi, 40 ${ }^{\circ} \mathrm{C}, 6.0 \% \mathrm{CH}_{3} \mathrm{OH}$ in $\mathrm{CO}_{2}, 3.0 \mathrm{~mL} / \mathrm{min}, 220 \mathrm{~nm}$ )

Analysis: $\quad \mathrm{C}_{17} \mathrm{H}_{36} \mathrm{O}_{4} \mathrm{Si}(332.55)$

Calcd: $\quad$ C, $61.40 ; \quad$ H, $10.91 \%$

Found; $\quad \mathrm{C}, 61.23 ; \quad \mathrm{H}, 11.12 \%$ 


\section{Preparation of Ethyl 8-(tert-Butyldimethylsilyl) 3-[N-(3,5-dinitrophenyl)carbamoyl]-2-} methyloctanoate (35)

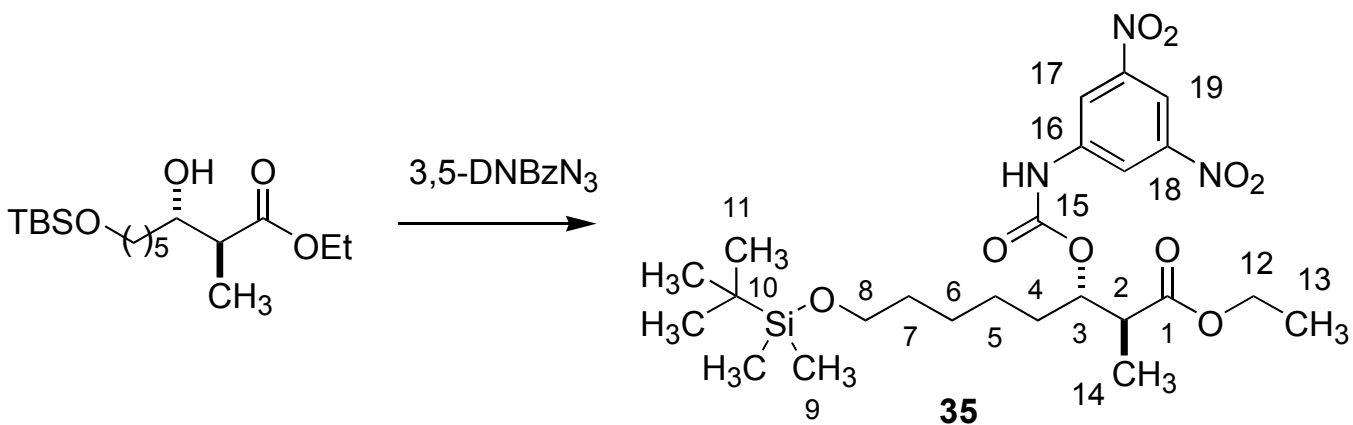

In a $10 \mathrm{~mL}$, 1-neck, round-bottom flask fitted with a reflux condenser a solution of $120 \mathrm{mg}$ of 3,5-dinitrobenzoylazide $\left(0.51 \mathrm{mmol}, 1.2\right.$ equiv) was heated at $120{ }^{\circ} \mathrm{C}$ for 20 minutes. A solution of ethyl 8-(tert-butyldimethylsilyl)-3-hydroxy-2-methyl octanoate 9bp $(0.42 \mathrm{mmol})$ in $1 \mathrm{~mL}$ toluene was then added and heating continued for $4 \mathrm{~h}$. The solution was then cooled to room temperature, washed with $5 \mathrm{~mL} 10 \%$ aqueous $\mathrm{HCl}, 5 \mathrm{~mL}$ saturated aqueous $\mathrm{NaHCO}_{3}$ and $5 \mathrm{~mL}$ brine prior to drying over $\mathrm{Na}_{2} \mathrm{SO}_{4}(1 \mathrm{~g})$, filtration and concentration in vacuo. Purification of the residue by silica gel column chromatography (hexanes/EtOAc, 8/2), afforded $150 \mathrm{mg}(66 \%)$ of 35 as a white crystalline solid.

Data for 35:

mp: $\quad 98-100^{\circ} \mathrm{C}$

${ }^{1} \mathrm{H}$ NMR: $\quad\left(400 \mathrm{MHz}, \mathrm{C}_{6} \mathrm{D}_{6}\right)$

8.14 (m, $1 \mathrm{H}, \mathrm{HC}(16)), 7.90$ (m, $2 \mathrm{H}, \mathrm{HC}(14)), 6.08$ (bs, $1 \mathrm{H}, \mathrm{NH}), 5.25$ (q, $J=$ 7.1, $1 \mathrm{H}, \mathrm{HC}(3)), 4.00$ (q, $\left.J=7.1,2 \mathrm{H}, \mathrm{H}_{2} \mathrm{C}(9)\right), 3.54$ (t, $J=6.4,2 \mathrm{H}, \mathrm{H}_{2} \mathrm{C}(8)$ ), 2.70 (pent, $J=7.1,1 \mathrm{H}, \mathrm{HC}(2))$, 1.62-1.40 (m, $8 \mathrm{H}, \mathrm{HC}(4,5,6,7)), 1.08$ (d, $J=7.1$, $\left.3 \mathrm{H}, \mathrm{H}_{3} \mathrm{C}(11)\right), 1.00$ (t, $\left.J=7.1,3 \mathrm{H}, \mathrm{H}_{3} \mathrm{C}(10)\right), 0.97$ (s, $\left.9 \mathrm{H}, \mathrm{H}_{3} \mathrm{C}(19)\right), 0.06$ (s, 6 $\left.\mathrm{H}, \mathrm{H}_{3} \mathrm{C}(17)\right)$

13 C NMR: $\quad\left(100 \mathrm{MHz}, \mathrm{C}_{6} \mathrm{D}_{6}\right)$

172.93 (C(1)), 152.31 (C(12)), 148.51 (C(15)), 140.02 (C(13)), 117.28 (C(14)), 112.33 (C(16)), 76.95 (C(3)), 63.00 (C(8)), 60.67 (C(9)), 43.61 (C(2)), 33.05 (C(4)), 31.84 (C(7)), 26.16 (C(5)), 26.09 (C(19)), 25.20 (C(6)), 18.46 (C(18)), 14.19 (C(10)), 13.19 (C(11), -5.21 (C(17))

TLC: $\quad R_{f} 0.65$ (hexanes/EtOAc, 8/2) $\left[\mathrm{UV}(254) / \mathrm{KMnO}_{4}\right]$ 
SFC: $\quad t_{R}(2 R, 3 R)-\mathbf{3 5}, 15.78(3.3 \%) ; t_{R}(2 S, 3 S)-\mathbf{3 5}, 16.62$ (96.7\%) (Whelk-O1, $125 \mathrm{psi}, 40$ ${ }^{\circ} \mathrm{C}, 6.0 \% \mathrm{CH}_{3} \mathrm{OH}$ in $\mathrm{CO}_{2}, 3.0 \mathrm{~mL} / \mathrm{min}, 220 \mathrm{~nm}$ )

\section{Preparation of Ethyl 8-Benzyloxy-3-hydroxy-2-methyloctanoate (9bq)}

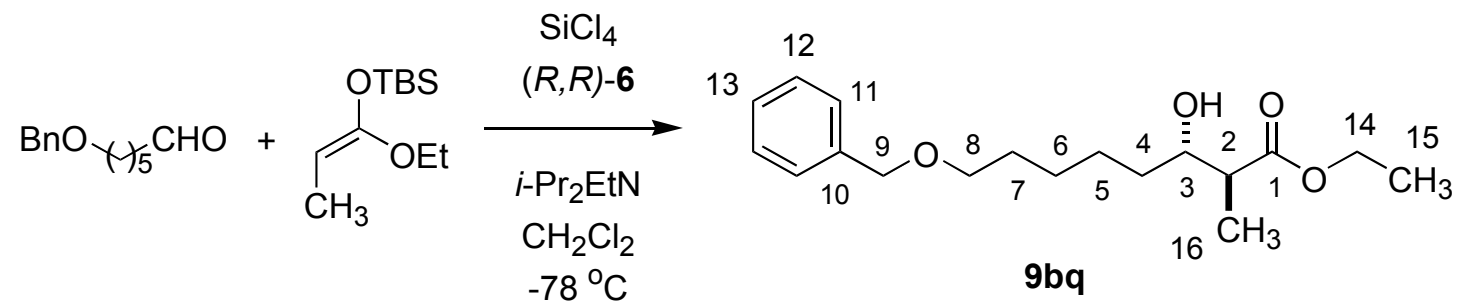

A flame-dried, $25 \mathrm{~mL}, 2$-neck, round-bottom flask containing a solution of $42 \mathrm{mg}$ of bisphosphoramide $(R, R)-6$ ( $0.05 \mathrm{mmol}, 0.05$ equiv), $8 \mu \mathrm{L}$ of $i-\operatorname{Pr}_{2} \operatorname{EtN}(0.05 \mathrm{mmol}, 0.05$ equiv) and $206 \mathrm{mg}$ of $\mathbf{5 q}(1.0 \mathrm{mmol})$ in $\mathrm{CH}_{2} \mathrm{Cl}_{2}(5 \mathrm{~mL})$ was cooled to $-78{ }^{\circ} \mathrm{C}$ under nitrogen. To the resulting solution was added $123 \mu \mathrm{L}$ of $\mathrm{SiCl}_{4}$ (1.1 mmol, 1.1 equiv). Then $260 \mathrm{mg}$ of $\mathbf{2 b}(1.2$ mmol, 1.2 equiv) was added dropwise over $5 \mathrm{~min}$ to the reaction mixture. The solution was allowed to stir at $-78{ }^{\circ} \mathrm{C}$ for $24 \mathrm{~h}$ whereupon the cold reaction mixture was poured into a rapidly stirring 1/1 sat. aq. $\mathrm{KF} / 1.0 \mathrm{M} \mathrm{KH}_{2} \mathrm{PO}_{4}$ solution $(20 \mathrm{~mL})$. This biphasic mixture was stirred vigorously for $1 \mathrm{~h}$ before filtration through Celite. The aqueous layer was washed with $\mathrm{CH}_{2} \mathrm{Cl}_{2}(3$ x $25 \mathrm{~mL})$. The combined organic extracts were washed with brine $(50 \mathrm{~mL})$, dried over $\mathrm{Na}_{2} \mathrm{SO}_{4}(2$ g), filtered and the filtrate was concentrated in vacuo. The residue was purified to yield, after silica gel column chromatography (hexanes/EtOAc, 8/2), $215 \mathrm{mg}$ (70 \%) of $\mathbf{9 b q}$ as a colorless oil.

Data for 9bq:

1 NMR: $\quad\left(400 \mathrm{MHz}, \mathrm{CDCl}_{3}\right)$

7.35 (m, $5 \mathrm{H}, \mathrm{HC}(11,12,13)), 4.49$ (s, $\left.2 \mathrm{H}, \mathrm{H}_{2} \mathrm{C}(9)\right), 4.16$ (q, $J=7.1,2 \mathrm{H}$, $\left.\mathrm{H}_{2} \mathrm{C}(14)\right), 3.63$ ( $\left.\mathrm{m}, 1 \mathrm{H}, \mathrm{HC}(3)\right), 3.46\left(\mathrm{t}, J=6.6 ., 2 \mathrm{H}, \mathrm{H}_{2} \mathrm{C}(8)\right), 2.59$ (d, $J=6.8,1$ H, OH), 2.49 (pent, $J=7.1,1 \mathrm{H}, \mathrm{HC}(2)), 1.62$ (pent, $\left.J=7.6,2 \mathrm{H}, \mathrm{H}_{2} \mathrm{C}(7)\right), 1.59-$ 1.37 (m, $6 \mathrm{H}, \mathrm{HC}(4,5,6)), 1.27$ (t, $\left.J=7.1,3 \mathrm{H}, \mathrm{H}_{3} \mathrm{C}(15)\right), 1.20$ (d, $J=7.1,3 \mathrm{H}$, $\left.\mathrm{H}_{3} \mathrm{C}(16)\right)$ 
${ }^{13} \mathrm{C}$ NMR: $\quad\left(100 \mathrm{MHz}, \mathrm{CDCl}_{3}\right)$

$176.10(\mathrm{C}(1)), 138.61(\mathrm{C}(10)), 128.33$ (C(12)), 127.61 (C(13)), 127.47 (C(11)),

$73.26(\mathrm{C}(3)), 72.87$ (C(9)), $70.31(\mathrm{C}(8)), 60.56(\mathrm{C}(14)), 45.12(\mathrm{C}(2)), 34.70$

(C(4)), $29.68(\mathrm{C}(7)), 26.13(\mathrm{C}(6)), 25.36(\mathrm{C}(5)), 14.41(\mathrm{C}(15)), 14.19(\mathrm{C}(16))$

IR: (neat)

3456 (m), 2930 (s), 2938 (s), 2859 (s), 1731 (s), 1496 (m), 1455 (s), 1372 (s),

$1259(\mathrm{~s}), 1184(\mathrm{~s}), 1100(\mathrm{~s}), 1028(\mathrm{~s})$

MS: $\quad(\mathrm{EI}, 70 \mathrm{eV})$

309 (M $\left.\mathrm{M}^{+1}, 3\right), 245$ (1), 199 (4), 184 (13), 153 (5), 131 (8), 115 (6), 102 (25), 91

(100), 74 (11)

Opt. Rot.: $\quad[\alpha]_{\mathrm{D}}^{24}+19.08(\mathrm{c}=1.0, \mathrm{EtOH})$

TLC: $\quad R_{f} 0.30$ (hexanes/EtOAc, $\left.8 / 2\right)\left[\mathrm{UV}(254) / \mathrm{KMnO}_{4}\right]$

SFC: $\quad t_{R}(2 R, 3 R)-9 \mathbf{b q}, 5.45(4.5 \%) ; t_{R}(2 S, 3 S)-9 \mathbf{b q}, 5.92$ (95.5\%) (Chiralpak OJ, 125 psi, $40{ }^{\circ} \mathrm{C}, 4.0 \% \mathrm{CH}_{3} \mathrm{OH}$ in $\mathrm{CO}_{2}, 3.0 \mathrm{~mL} / \mathrm{min}, 220 \mathrm{~nm}$ )

Analysis: $\quad \mathrm{C}_{18} \mathrm{H}_{28} \mathrm{O}_{4}(308.41)$

Calcd: $\quad$ C, $70.10 ; \quad$ H, $9.15 \%$

Found; $\quad$ C, 69.86; H, $9.38 \%$

Preparation of Ethyl 8-Benzoyl-3-hydrox-2-methyloctanoate (9br)

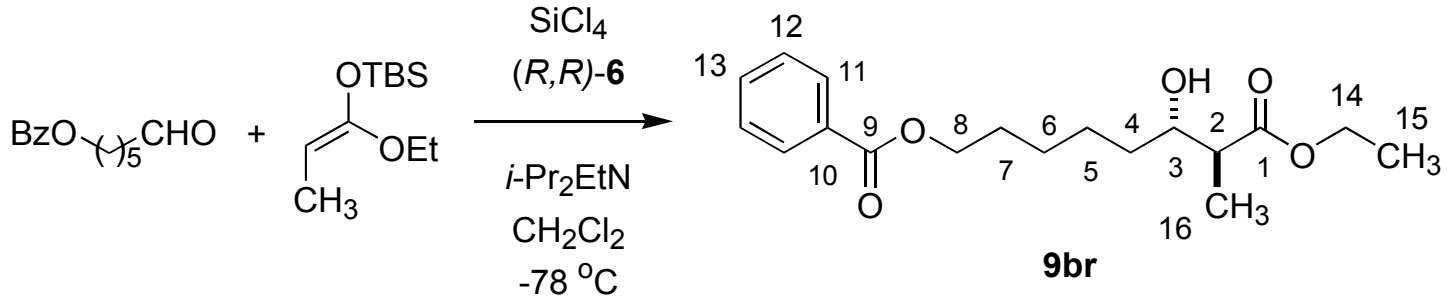

A flame-dried, $25 \mathrm{~mL}, 2$-neck, round-bottom flask containing a solution of $42 \mathrm{mg}$ of bisphosphoramide $(R, R)-6$ ( $0.05 \mathrm{mmol}, 0.05$ equiv), $8 \mu \mathrm{L}$ of $i-\operatorname{Pr}_{2} \operatorname{EtN}(0.05 \mathrm{mmol}, 0.05$ equiv) and $220 \mathrm{mg}$ of $5 \mathbf{r}(1.0 \mathrm{mmol})$ in $\mathrm{CH}_{2} \mathrm{Cl}_{2}(5 \mathrm{~mL})$ was cooled to $-78{ }^{\circ} \mathrm{C}$ under nitrogen. To the resulting solution was added $123 \mu \mathrm{L}$ of $\mathrm{SiCl}_{4}(1.1 \mathrm{mmol}, 1.1$ equiv). Then $260 \mathrm{mg}$ of $\mathbf{2 b}(1.2$ mmol, 1.2 equiv) was added dropwise over 5 min to the reaction mixture. The solution was allowed to stir at $-78{ }^{\circ} \mathrm{C}$ (bath temperature) for $24 \mathrm{~h}$ whereupon the cold reaction mixture was 
poured into a rapidly stirring $1 / 1$ sat. aq. $\mathrm{KF} / 1.0 \mathrm{M} \mathrm{KH}_{2} \mathrm{PO}_{4}$ solution $(20 \mathrm{~mL})$. This biphasic mixture was stirred vigorously for $1 \mathrm{~h}$ before filtration through Celite. The aqueous layer was washed with $\mathrm{CH}_{2} \mathrm{Cl}_{2}(3 \times 25 \mathrm{~mL})$. The combined organic extracts were washed with brine (50 $\mathrm{mL})$, dried over $\mathrm{Na}_{2} \mathrm{SO}_{4}(2 \mathrm{~g})$, filtered and the filtrate was concentrated in vacuo. The residue was purified to yield, after silica gel column chromatography (hexanes/EtOAc, 4/1), $193 \mathrm{mg}$ (60 $\%)$ of $9 \mathrm{br}$ as a colorless oil. Enantiomeric analysis was determined on the 3,5dinitrophenylcarbamate 36 .

\section{Data for 9br:}

${ }^{1} \mathrm{H}$ NMR: $\quad\left(400 \mathrm{MHz}, \mathrm{CDCl}_{3}\right)$

8.04 (m, $2 \mathrm{H}, \mathrm{HC}(11)), 7.55$ (m, $1 \mathrm{H}, \mathrm{HC}(13)), 7.42$ (m, $2 \mathrm{H}, \mathrm{HC}(12)), 4.32$ (t, $J=$ 6.6, 2 H, $\mathrm{H}_{2} \mathrm{C}(8)$ ), 4.16 (q, $\left.J=7.1,2 \mathrm{H}, \mathrm{H}_{2} \mathrm{C}(14)\right), 3.65$ (m, $\left.1 \mathrm{H}, \mathrm{HC}(3)\right), 2.64$ (d, $J$ $=6.8,1 \mathrm{H}, \mathrm{OH}$ ), 2.50 (pent, $J=7.1,1 \mathrm{H}, \mathrm{HC}(2)), 1.78$ (pent, $J=6.8,2 \mathrm{H}$, $\left.\mathrm{H}_{2} \mathrm{C}(7)\right), 1.66-1.44(\mathrm{~m}, 6 \mathrm{H}, \mathrm{HC}(4,5,6)), 1.27$ (t, $\left.J=7.1,3 \mathrm{H}, \mathrm{H}_{3} \mathrm{C}(15)\right), 1.21$ (d, $\left.J=7.3,3 \mathrm{H}, \mathrm{H}_{3} \mathrm{C}(16)\right)$

${ }^{13} \mathrm{C} \mathrm{NMR}: \quad\left(100 \mathrm{MHz}, \mathrm{CDCl}_{3}\right)$ 176.08 (C(1)), $166.65(\mathrm{C}(9)), 132.81(\mathrm{C}(13)), 130.42$ (C(10)), 129.51 (C(11)), $128.31(\mathrm{C}(12)), 73.70(\mathrm{C}(3)), 64.94(\mathrm{C}(8)), 60.59(\mathrm{C}(14)), 45.13$ (C(2)), 34.65 (C(4)), $28.71(\mathrm{C}(7)), 26.03$ (C(6)), $25.27(\mathrm{C}(5)), 14.41$ (C(15)), 14.19 (C(16)) IR: (neat) $3513(\mathrm{~m}), 2979(\mathrm{~m}), 2940(\mathrm{~s}), 2861(\mathrm{~m}), 1719(\mathrm{~s}), 1602(\mathrm{w}), 1452(\mathrm{~s}), 1376(\mathrm{~m})$, $1315(\mathrm{~m}), 1276(\mathrm{~s}), 1176(\mathrm{~s}), 1114(\mathrm{~s}), 1070$ (s), 1026 (s)

MS: $\quad(E I, 70 \mathrm{eV})$ $322\left(\mathrm{M}^{+}, 1\right), 258(3), 221(8), 192(14), 123(58), 105$ (100), 77 (34)

Opt. Rot.: $\quad[\alpha]_{\mathrm{D}}^{24}-2.40(\mathrm{c}=1.0, \mathrm{EtOH})$

TLC: $\quad R_{f} 0.27$ (hexanes/EtOAc, 8/2) [UV(254)/KMnO 4$]$

SFC: $\quad t_{R}(2 R, 3 R)-\mathbf{3 6}, 14.07(5.0 \%) ; t_{R}(2 S, 3 S)$-36, 14.75 (95.0\%) (Whelk-O1, $150 \mathrm{psi}, 40$ ${ }^{\circ} \mathrm{C}, 14.0 \% \mathrm{CH}_{3} \mathrm{OH}$ in $\mathrm{CO}_{2}, 2.0 \mathrm{~mL} / \mathrm{min}, 220 \mathrm{~nm}$ )

Analysis: $\quad \mathrm{C}_{18} \mathrm{H}_{26} \mathrm{O}_{5}(322.40)$

Calcd: $\quad$ C, $67.06 ; \quad \mathrm{H}, 8.43 \%$

Found; $\quad$ C, $66.77 ; \quad \mathrm{H}, 8.24 \%$ 
Preparation of Ethyl 8-Benzoyl-3-[N-(3,5-dinitrophenyl)carbamoyl]octanoate (36)

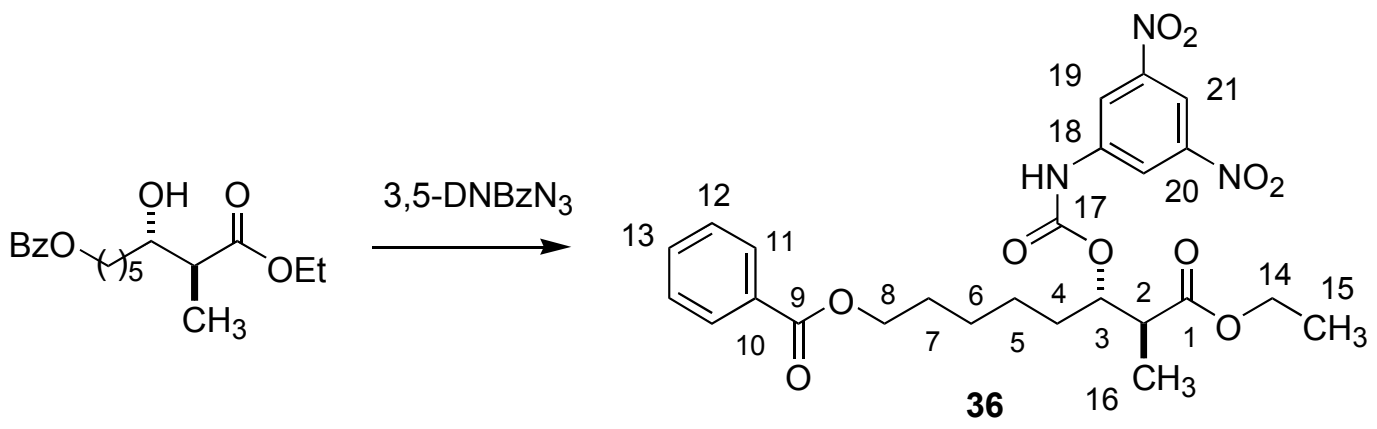

In a $10 \mathrm{~mL}$, 1-neck, round-bottom flask fitted with a reflux condenser a solution of $84 \mathrm{mg}$ of 3,5dinitrobenzoylazide ( $0.36 \mathrm{mmol}, 1.2$ equiv) in $5 \mathrm{~mL}$ of toluene was heated at $120{ }^{\circ} \mathrm{C}$ for 20 minutes. A solution of $9 \mathrm{br}(0.29 \mathrm{mmol})$ in $1 \mathrm{~mL}$ toluene was then added and heating continued for $4 \mathrm{~h}$. The solution was then cooled to room temperature, washed with $5 \mathrm{~mL} 10 \%$ aqueous $\mathrm{HCl}, 5 \mathrm{~mL}$ saturated aqueous $\mathrm{NaHCO}_{3}$ and $5 \mathrm{~mL}$ brine prior to drying over $\mathrm{Na}_{2} \mathrm{SO}_{4}(1 \mathrm{~g})$, filtration and concentration in vacuo. Purification of the residue by silica gel column chromatography (hexanes/EtOAc, 8/2), afforded $121 \mathrm{mg}$ (78\%) of $\mathbf{3 6}$ as a white crystalline solid. Data for 36:

mp: $\quad 116-118^{\circ} \mathrm{C}$

근 NMR: $\quad\left(400 \mathrm{MHz}, \mathrm{C}_{6} \mathrm{D}_{6}\right)$

8.25 (m, 3 H, HC(20,21)), 8.17 (m, 2 H, HC(19)), 7.14 (m, 3 H, HC(14,16)), 6.72

(bs, $1 \mathrm{H}, \mathrm{NH}$ ), 5.36 (m, $1 \mathrm{H}, \mathrm{HC}(3)$ ), 4.29 (m, $2 \mathrm{H}, \mathrm{H}_{2} \mathrm{C}(8)$ ), 4.11 (dq, J= 2.7, 7.1, 2 H, $\mathrm{H}_{2} \mathrm{C}(9)$ ), 2.85 (pent, $\left.J=7.1,1 \mathrm{H}, \mathrm{HC}(2)\right)$, 1.67-1.29 (m, $8 \mathrm{H}, \mathrm{HC}(4,5,6,7)$ ),

$1.20\left(\mathrm{~d}, J=7.1,3 \mathrm{H}, \mathrm{H}_{3} \mathrm{C}(11)\right), 1.10$ (t, $\left.J=3 \mathrm{H}, \mathrm{H}_{3} \mathrm{C}(10)\right)$

${ }^{13} \mathrm{C}$ NMR: $\quad\left(100 \mathrm{MHz}, \mathrm{C}_{6} \mathrm{D}_{6}\right)$

$173.01(\mathrm{C}(1)), 166.60$ (C(17)), 152.45 (C(12)), 148.56 (C(15)), 140.25 (C(13)), 133.02 (C(21)), 130.82 (C(18)), 129.79 (C(19)), 128.61 (C(20)), 117.40 (C(14)), $112.35(\mathrm{C}(16)), 76.85(\mathrm{C}(3)), 64.70(\mathrm{C}(8)), 60.78(\mathrm{C}(9)), 43.61(\mathrm{C}(2)), 31.30$ (C(4)), 28.69 (C(7)), 25.88 (C(6)), 24.74 (C(5)), 14.17 (C(10)), 13.10 (C(11))

TLC: $\quad R_{f} 0.37$ (hexanes/EtOAc, 8/2) [UV(254)/KMnO 4$]$

SFC: $\quad t_{R}(2 R, 3 R)-36,14.07(5.0 \%) ; t_{R}(2 S, 3 S)-36,14.75$ (95.0\%) (Whelk-O1, 150 psi, 40 ${ }^{\circ} \mathrm{C}, 14.0 \% \mathrm{CH}_{3} \mathrm{OH}$ in $\mathrm{CO}_{2}, 2.0 \mathrm{~mL} / \mathrm{min}, 220 \mathrm{~nm}$ ) 


\section{General Procedure 6. Addition of Silyl Dienol Ethers to Conjugated Aldehydes.}

Preparation of $(2 E, 5 R)$ Ethyl 5-Hydroxy-5-phenyl-2-pentenoate ((2E,5R)-14aa)

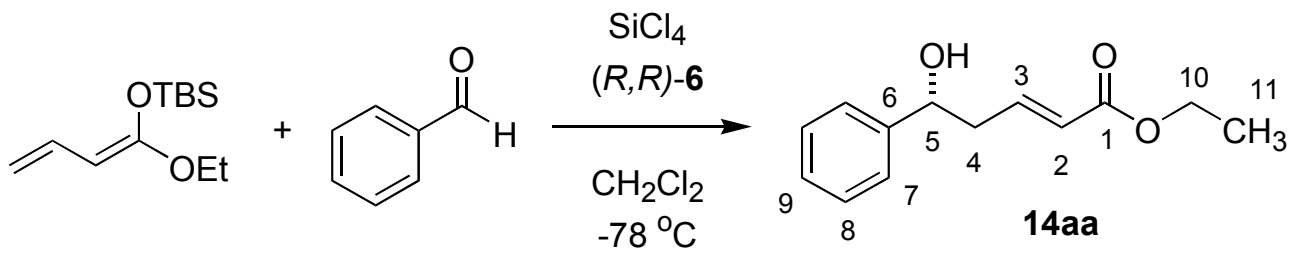

A flame-dried, $25 \mathrm{~mL}, 2$-neck, round-bottom flask containing a solution of $8.4 \mathrm{mg}$ of bisphosphoramide $(R, R)-6(0.01 \mathrm{mmol}, 0.01$ equiv) and $102 \mu \mathrm{L}$ of benzaldehyde $5 \mathbf{a}(1.0 \mathrm{mmol})$ in $\mathrm{CH}_{2} \mathrm{Cl}_{2}(5 \mathrm{~mL})$ was cooled to $-78{ }^{\circ} \mathrm{C}$ under nitrogen in a dry ice/isopropanol bath. To the resulting solution was added $123 \mu \mathrm{L}$ of $\mathrm{SiCl}_{4}(1.1 \mathrm{mmol}, 1.1$ equiv). Then $272 \mathrm{mg}$ of $4 \mathbf{a}(1.2$ mmol, 1.2 equiv) was added dropwise over $5 \mathrm{~min}$ to the reaction mixture. The solution was allowed to stir at $-78{ }^{\circ} \mathrm{C}$ for $3 \mathrm{~h}$ in a dry ice/isopropanol bath whereupon the cold reaction mixture was poured into a rapidly stirring 1/1 sat. aq. $\mathrm{KF} / 1.0 \mathrm{M} \mathrm{KH}_{2} \mathrm{PO}_{4}$ solution $(20 \mathrm{~mL})$. This biphasic mixture was stirred vigorously for $1 \mathrm{~h}$ before filtration through Celite. The aqueous layer was washed with $\mathrm{CH}_{2} \mathrm{Cl}_{2}(3 \times 25 \mathrm{~mL})$. The combined organic extracts were washed with brine $(50 \mathrm{~mL})$, dried over $\mathrm{Na}_{2} \mathrm{SO}_{4}(2 \mathrm{~g})$, filtered and the filtrate was concentrated in vacuo. The residue was purified by silica gel column chromatography (hexanes/EtOAc, 4/1) to yield $196 \mathrm{mg}$ $(89 \%)$ of $(2 E, 5 R)$-14aa as a colorless oil. NMR spectral data matched that previously reported in the literature. ${ }^{23}$

Data for $(2 E, 5 R)-14 a a:$

1 NMR: $\quad\left(400 \mathrm{MHz}, \mathrm{CDCl}_{3}\right)$

7.4-7.25 (m, $5 \mathrm{H}, \mathrm{HC}(7,8,9)), 6.95(\mathrm{dt}, J=15.6,7.1,1 \mathrm{H}, \mathrm{HC}(3)), 5.87(\mathrm{~d}, J=$ 15.6, $1 \mathrm{H}, \mathrm{HC}(2)), 4.81$ (m, $1 \mathrm{H}, \mathrm{HC}(5)), 4.16$ (q, $\left.J=7.1,2 \mathrm{H}, \mathrm{H}_{2} \mathrm{C}(10)\right)$ ), 2.64 (m, $\left.2 \mathrm{H}, \mathrm{H}_{2} \mathrm{C}(4)\right), 2.2$ (bs, $\left.1 \mathrm{H}, \mathrm{OH}\right), 1.26$ (t, $\left.J=7.1,3 \mathrm{H}, \mathrm{H}_{3} \mathrm{C}(11)\right)$

${ }^{13} \mathrm{C} \mathrm{NMR}: \quad\left(100 \mathrm{MHz}, \mathrm{CDCl}_{3}\right)$

166.29 (C(1)), 144.67 (C(3)), 142.97 (C(6)), 128.60 (C(8)), 127.89 (C(9)), 125.69 (C(7)), 124.0 (C(2)), 73.06 (C(5)), 60.29 (C(10)), 41.83 (C(4)), 14.21 (C(11))

IR: (neat)

3440 (m), 3029 (w), 2983 (m), 2939 (w), 2904 (w), 1712 (s), 1654 (s), 1494 (m), 1454 (m), 1369 (m), 1315 (s), 1278 (s), 1199 (s), 1160 (s), 1043 (s) 
MS: $\quad(E I, 70 \mathrm{eV})$

$221\left(\mathrm{M}^{+1}, 3\right), 203$ (32), 175 (3), 129 (11), 114 (100), 107 (87), 86 (37)

Opt. Rot.: $\quad[\alpha]_{\mathrm{D}}^{24}+22.86(\mathrm{c}=1.0, \mathrm{EtOH})$, lit. $[\alpha]_{\mathrm{D}}^{24}+10.4(\mathrm{c}=1.13, \mathrm{EtOH})$ for $(5 R)-\mathbf{X X}^{23}$

TLC: $\quad R_{f} 0.26$ (hexanes/EtOAc, $\left.4 / 1\right)\left[\mathrm{UV}(254) / \mathrm{KMnO}_{4}\right]$

SFC: $\quad t_{R}(R)$-14aa, 3.25 (98.8\%); $t_{R}(S)$-14aa, 4.17 (1.2\%) (Chiralpak AD, 125 psi, 40 ${ }^{\circ} \mathrm{C}, 10.0 \% \mathrm{CH}_{3} \mathrm{OH}$ in $\mathrm{CO}_{2}, 3.0 \mathrm{~mL} / \mathrm{min}, 220 \mathrm{~nm}$ )

Analysis: $\quad \mathrm{C}_{13} \mathrm{H}_{16} \mathrm{O}_{3}(220.26)$

Calcd: $\quad$ C, $70.89 ; \quad$ H, $7.32 \%$

Found: $\quad$ C, $70.64 ; \quad \mathrm{H}, 7.28 \%$

Preparation of $(2 E, 5 R, 6 E)$ Ethyl 5-Hydroxy-7-phenyl-2,6-heptadienoate $((2 E, 5 R, 6 E)-14 a g)$

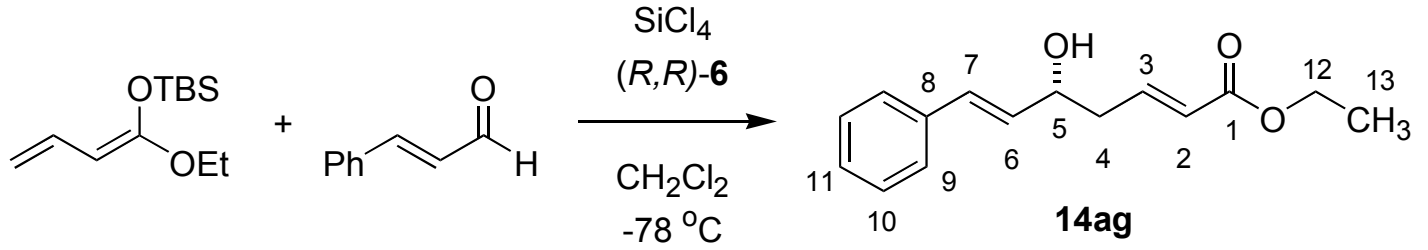

Following General Procedure 6, $8.4 \mathrm{mg}$ of bis-phosphoramide $(R, R)-6$ ( $0.01 \mathrm{mmol}, 0.01$ equiv) was combined with $126 \mu \mathrm{L}$ of cinnamaldehyde $5 \mathrm{~g}(1.0 \mathrm{mmol}), 123 \mu \mathrm{L}$ of $\mathrm{SiCl}_{4}(1.1 \mathrm{mmol}, 1.1$ equiv) and $272 \mathrm{mg}$ of $4 \mathrm{a}$ (1.2 mmol, 1.2 equiv) to yield, after silica gel column chromatography (silica gel, hexanes/EtOAc, 4/1), $207 \mathrm{mg}(84 \%)$ of $(2 E, 5 R, 6 E)$-14ag as a colorless oil.

Data for $(2 E, 5 R, 6 E)-\mathbf{1 4 a g}$ :

${ }^{1} \mathrm{H}$ NMR: $\quad\left(400 \mathrm{MHz}, \mathrm{CDCl}_{3}\right)$

7.4-7.2 (m, $5 \mathrm{H}, \mathrm{HC}(9,10,11)), 7.0(\mathrm{dt}, J=15.6,7.3,1 \mathrm{H}, \mathrm{HC}(3)), 6.62(\mathrm{~d}, J=$ 16.1, $1 \mathrm{H}, \mathrm{HC}(7)), 6.23$ (dd, $J=15.9,6.6,1 \mathrm{H}, \mathrm{HC}(6)), 5.94$ (dt, $J=15.6,1.5,1$ H, $\mathrm{HC}(2)), 4.45$ (q, $J=6.1,1 \mathrm{H}, \mathrm{HC}(5)), 4.18$ (q, $\left.J=7.1,2 \mathrm{H}, \mathrm{H}_{2} \mathrm{C}(12)\right), 2.55$ (t, $J$ $\left.=7.1,2 \mathrm{H}, \mathrm{H}_{2} \mathrm{C}(4)\right), 1.28\left(\mathrm{t}, J=7.08,3 \mathrm{H}, \mathrm{H}_{3} \mathrm{C}(13)\right)$

${ }^{13} \mathrm{C} \mathrm{NMR}: \quad\left(100 \mathrm{MHz}, \mathrm{CDCl}_{3}\right)$

$166.24(\mathrm{C}(1)), 144.17(\mathrm{C}(3)), 136.24(\mathrm{C}(8)), 131.13(\mathrm{C}(7)), 130.87$ (C(6)), 128.61 $(\mathrm{C}(9)), 127.92(\mathrm{C}(11)), 126.54(\mathrm{C}(10)), 124.24(\mathrm{C}(2)), 71.53$ (C(5)), 60.33 (C(12)), $40.12(\mathrm{C}(4)), 14.23(\mathrm{C}(13))$ 
IR: (neat)

3430 (m), 3027 (w), 2983 (m), 2937 (w), 2904 (w), 1716 (s), 1654 (m), 1494 (w), 1448 (w), 1369 (m), 1317 (m), 1272 (m), 1201 (m), 1164 (m), 1041 (m)

MS: $\quad(\mathrm{EI}, 70 \mathrm{eV})$

$245\left(\mathrm{M}^{-1}, 3\right), 229$ (100), 183 (11), 155 (17), 133 (31), 115 (11)

Opt. Rot.: $\quad[\alpha]_{\mathrm{D}}^{24}-17.86(\mathrm{c}=1.0, \mathrm{EtOH})$

TLC: $\quad R_{f} 0.25$ (hexanes/EtOAc, 4/1) [UV(254)/KMnO $\left.\mathrm{KM}_{4}\right]$

SFC: $\quad t_{R}(R)-14 a g, 4.15(98.3 \%) ; t_{R}(S)$-14ag, $4.41(1.7 \%)$ (Chiralpak AD, 125 psi, 40 ${ }^{\circ} \mathrm{C}, 10.0 \% \mathrm{CH}_{3} \mathrm{OH}$ in $\mathrm{CO}_{2}, 3.0 \mathrm{~mL} / \mathrm{min}, 220 \mathrm{~nm}$ )

Analysis: $\quad \mathrm{C}_{15} \mathrm{H}_{18} \mathrm{O}_{3}(246.30)$

Calcd: $\quad$ C, $73.15 ; \quad$ H, $7.37 \%$

Found: $\quad$ C, $72.91 ; \quad H, 7.38 \%$

General Procedure 7. Addition of Silyl Dienol Ethers to Aliphatic Aldehydes. Preparation of $(2 E, 5 S)$ Ethyl 5-Hydroxy-7-phenyl-2-heptenoate ((2E,5S)-14ak)

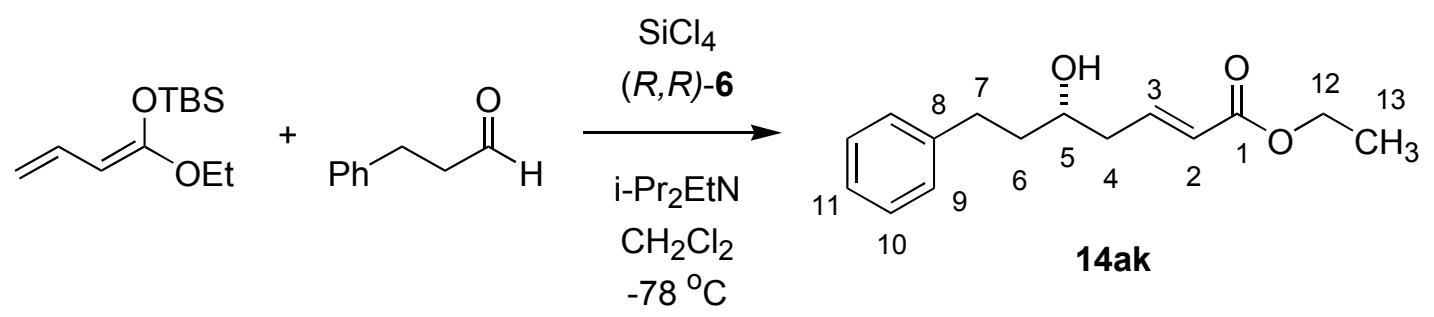

A flame-dried, $25 \mathrm{~mL}, 2$-neck flask containing a solution of $42 \mathrm{mg}$ of bis-phosphoramide $(R, R)-6$ (0.05 mmol, 0.05 equiv), $8 \mu \mathrm{L}$ of $i-\operatorname{Pr}_{2} \operatorname{EtN}(0.05 \mathrm{mmol}, 0.05$ equiv) and $132 \mu \mathrm{L}$ of hydrocinnamaldehyde 5k $(1.0 \mathrm{mmol})$ in $\mathrm{CH}_{2} \mathrm{Cl}_{2}(5 \mathrm{~mL})$ was cooled to $-78{ }^{\circ} \mathrm{C}$ under nitrogen. To the resulting solution was added $123 \mu \mathrm{L}$ of $\mathrm{SiCl}_{4}(1.1 \mathrm{mmol}, 1.1$ equiv). Then $272 \mathrm{mg}$ of $4 \mathbf{a}(1.2$ mmol, 1.2 equiv) was added dropwise over $5 \mathrm{~min}$ to the reaction mixture. The solution was allowed to stir at $-78{ }^{\circ} \mathrm{C}$ for $24 \mathrm{~h}$ whereupon the cold reaction mixture was poured into a rapidly stirring $1 / 1$ sat. aq. $\mathrm{KF} / 1.0 \mathrm{M} \mathrm{KH}_{2} \mathrm{PO}_{4}$ solution $(20 \mathrm{~mL})$.. This biphasic mixture was stirred vigorously for $1 \mathrm{~h}$ before filtration through Celite. The aqueous layer was washed with $\mathrm{CH}_{2} \mathrm{Cl}_{2}$ (3 x $25 \mathrm{~mL})$. The combined organic extracts were washed with brine $(50 \mathrm{~mL})$, dried over $\mathrm{Na}_{2} \mathrm{SO}_{4}(2$ $\mathrm{g}$ ), filtered and the filtrate was concentrated in vacuo. The residue was purified by silica gel 
column chromatography (hexanes/EtOAc, 4/1) to yield $167 \mathrm{mg}(68 \%)$ of $(2 E, 5 S)$-14ak as a colorless oil. NMR spectral data matched that previously reported in the literature. ${ }^{24}$

Data for $(2 E, 5 S)-\mathbf{1 4 a k}$ :

${ }^{1} \mathrm{H}$ NMR: $\quad\left(500 \mathrm{MHz}, \mathrm{CDCl}_{3}\right)$

7.3-7.18 (m, $5 \mathrm{H}, \mathrm{HC}(9,10,11)), 6.96(\mathrm{dt}, J=15.6,7.7,1 \mathrm{H}, \mathrm{HC}(3)), 5.9$ (dt, $J=$ 15.6, 1.5, $1 \mathrm{H}, \mathrm{HC}(2)), 4.19$ (q, $J=7.3,2 \mathrm{H}, \mathrm{H}_{2} \mathrm{C}(12)$ ), 3.78 (m, $1 \mathrm{H}, \mathrm{HC}(5)$ ), 2.80-2.69 (m, $\left.2 \mathrm{H}, \mathrm{H}_{2} \mathrm{C}(4)\right)$, 2.42-2.35 (m, $\left.2 \mathrm{H}, \mathrm{H}_{2} \mathrm{C}(7)\right)$, 1.81 (m, $2 \mathrm{H}, \mathrm{H}_{2} \mathrm{C}(6)$ ), $1.28\left(\mathrm{t}, J=7.1,3 \mathrm{H}, \mathrm{H}_{3} \mathrm{C}(13)\right)$

${ }^{13} \mathrm{C} \mathrm{NMR}: \quad\left(125 \mathrm{MHz}, \mathrm{CDCl}_{3}\right)$

$166.27(\mathrm{C}(1)), 144.27(\mathrm{C}(3)), 141.58(\mathrm{C}(8)), 128.49(\mathrm{C}(9)), 128.41(\mathrm{C}(10))$, $125.99(\mathrm{C}(11)), 124.13(\mathrm{C}(2)), 69.79(\mathrm{C}(5)), 60.31(\mathrm{C}(12)), 40.31(\mathrm{C}(4)), 38.64$ $(\mathrm{C}(7)), 31.92(\mathrm{C}(6)), 14.20(\mathrm{C}(13))$

IR: (neat)

$3442(\mathrm{~m}), 3025(\mathrm{w}), 2981(\mathrm{w}), 2935(\mathrm{~m}), 2863(\mathrm{w}), 1718(\mathrm{~s}), 1654(\mathrm{~m}), 1496(\mathrm{~m})$, $1454(\mathrm{~m}), 1369(\mathrm{~m}), 1319(\mathrm{~m}), 1270(\mathrm{~s}), 1211(\mathrm{~m}), 1176(\mathrm{~s}), 1045(\mathrm{~s})$

MS: $\quad(\mathrm{EI}, 70 \mathrm{eV})$ $249\left(\mathrm{M}^{+1}, 1\right), 230$ (15), 203 (5), 156 (7), 135 (7), 114 (80), 91 (100)

Opt. Rot.: $\quad[\alpha]_{\mathrm{D}}^{24}-12.77(\mathrm{c}=1.0, \mathrm{EtOH})$

TLC: $\quad R_{f} 0.21$ (hexanes/EtOAc, $\left.4 / 1\right)\left[\mathrm{UV}(254) / \mathrm{KMnO}_{4}\right]$

SFC: $\quad t_{R}(R)$-14ak, 7.95 (4.7\%); $t_{R}(S)$-14ak, 8.29 (95.3\%) (Chiralpak AD, 125 psi, 40 ${ }^{\circ} \mathrm{C}, 7.0 \% \mathrm{CH}_{3} \mathrm{OH}$ in $\left.\mathrm{CO}_{2}, 2.0 \mathrm{~mL} / \mathrm{min}, 220 \mathrm{~nm}\right)$

Analysis: $\quad \mathrm{C}_{15} \mathrm{H}_{20} \mathrm{O}_{3}(248.32)$

Calcd: $\quad$ C, $72.55 ; \quad \mathrm{H}, 8.12 \%$

Found: $\quad$ C, $72.44 ; \quad \mathrm{H}, 8.23 \%$ 
Preparation of (2E,5R) Methyl 5-Hydroxy-2-methyl-5-phenyl-2-pentenoate ((2E,5R)-14ba)

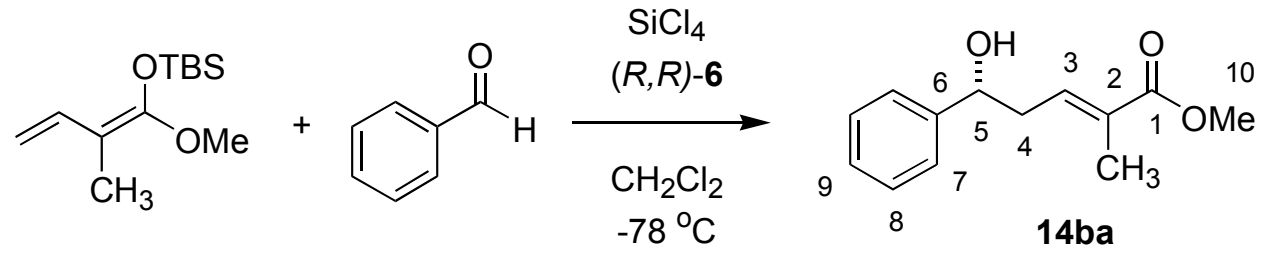

Following General Procedure 6, $8.4 \mathrm{mg}$ of bis-phosphoramide $(R, R)-6$ ( $0.01 \mathrm{mmol}, 0.01$ equiv) was combined with $102 \mu \mathrm{L}$ of benzaldehyde $\mathbf{5 a}(1.0 \mathrm{mmol}), 123 \mu \mathrm{L}$ of $\mathrm{SiCl}_{4}(1.1 \mathrm{mmol}, 1.1$ equiv) and $272 \mathrm{mg}$ of $\mathbf{4 b}$ (1.2 mmol, 1.2 equiv) to yield, after silica gel column chromatography (hexanes/EtOAc, 4/1), $205 \mathrm{mg}$ (93\%) of (2E,5R)-14ba as a colorless oil.

\section{Data for $(2 E, 5 R)-\mathbf{1 4 b a}$ :}

l. $\mathrm{H}$ NMR: $\quad\left(500 \mathrm{MHz}, \mathrm{CDCl}_{3}\right)$

7.4-7.28 (m, $5 \mathrm{H}, \mathrm{HC}(7,8,9)), 6.83$ (t, $J=7.5,1 \mathrm{H}, \mathrm{HC}(3)), 4.83$ (dd, $J=7.7,5.2$, $1 \mathrm{H}, \mathrm{HC}(5)), 3.72$ (s, $\left.3 \mathrm{H}, \mathrm{H}_{3} \mathrm{C}(10)\right)$, 2.71-2.56 (m, $\left.2 \mathrm{H}, \mathrm{H}_{2} \mathrm{C}(4)\right), 2.0$ (s, $1 \mathrm{H}, \mathrm{OH}$ ), $1.79\left(\mathrm{~s}, 3 \mathrm{H}, \mathrm{H}_{3} \mathrm{C}(11)\right)$

${ }^{13} \mathrm{C} \mathrm{NMR}: \quad\left(125 \mathrm{MHz}, \mathrm{CDCl}_{3}\right)$

168.37 (C(1)), 143.17 (C(6)), 137.68 (C(3)), 129.95 (C(8)), 128.60 (C(9)), 127.88 $(\mathrm{C}(7)), 125.71(\mathrm{C}(2)), 73.46(\mathrm{C}(5)), 51.76(\mathrm{C}(10)), 38.43(\mathrm{C}(4)), 12.58(\mathrm{C}(11))$

IR: (neat)

$3453(\mathrm{~m}), 3029$ (w), 2950 (m), 1710 (s), 1648 (m), 1436 (s), 1278 (s), 1195 (s), 1124 (s), 1083 (s), 1051 (s)

MS: $\quad(\mathrm{EI}, 70 \mathrm{eV})$

$221\left(\mathrm{M}^{+1}, 15\right), 203$ (100), 171 (20), 143 (6), 114 (30)

Opt. Rot.: $\quad[\alpha]_{\mathrm{D}}^{24}+2.51(\mathrm{c}=1.0, \mathrm{EtOH})$

TLC: $\quad R_{f} 0.22$ (hexanes/EtOAc, $\left.4 / 1\right)\left[\mathrm{UV}(254) / \mathrm{KMnO}_{4}\right]$

SFC: $\quad t_{R}(S)$-14ba, $4.17(0.5 \%) ; t_{R}(R)-14 \mathbf{b a}, 5.33$ (99.5\%) (Chiralpak AD, 125 psi, 40 ${ }^{\circ} \mathrm{C}, 7.0 \% \mathrm{CH}_{3} \mathrm{OH}$ in $\left.\mathrm{CO}_{2}, 3.0 \mathrm{~mL} / \mathrm{min}, 220 \mathrm{~nm}\right)$

Analysis: $\quad \mathrm{C}_{13} \mathrm{H}_{16} \mathrm{O}_{3}(220.20)$

Calcd: $\quad$ C, $70.89 ; \quad$ H, $7.32 \%$

Found: $\quad$ C, $70.74 ; \quad$ H, $7.31 \%$ 
Preparation of $(2 E, 6 E)$ Methyl 5-Hydroxy-2-methyl-7-phenyl-2,6-heptadienoate $((2 E, 6 E)$ 14bg)

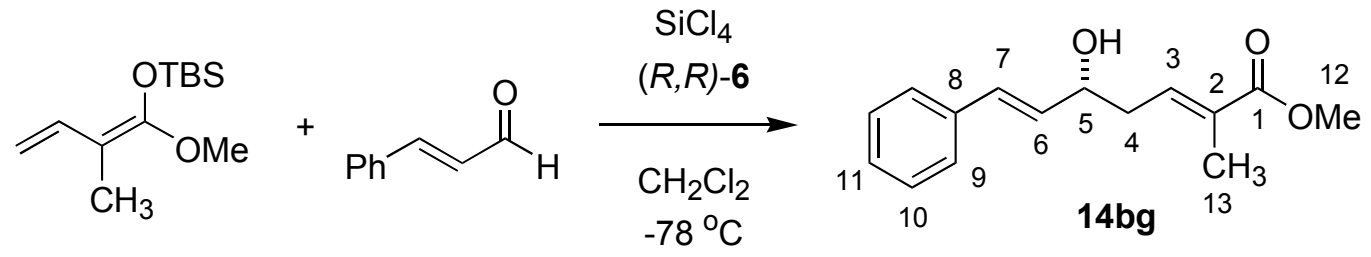

Following General Procedure 6, $8.4 \mathrm{mg}$ of bis-phosphoramide $(R, R)-6$ (0.01 mmol, 0.01 equiv) was combined with $126 \mu \mathrm{L}$ of cinnamaldehyde $\mathbf{5 g}(1.0 \mathrm{mmol}), 123 \mu \mathrm{L}$ of $\mathrm{SiCl}_{4}(1.1 \mathrm{mmol}, 1.1$ equiv) and $272 \mathrm{mg}$ of $\mathbf{4 b}$ (1.2 mmol, 1.2 equiv) to yield, after silica gel column chromatography (hexanes/EtOAc, 4/1), $216 \mathrm{mg}(88 \%)$ of $(2 E, 6 E)-\mathbf{1 4 b g}$ as a colorless oil.

Data for $(2 E, 6 E)-\mathbf{1 4 b g}$ :

${ }^{1} \mathrm{H}$ NMR: $\quad\left(500 \mathrm{MHz}, \mathrm{CDCl}_{3}\right)$

7.4-7.2 (m, $5 \mathrm{H}, \mathrm{HC}(9,10,11)), 6.85$ (t, $J=7.5,1 \mathrm{H}, \mathrm{HC}(3)), 6.63$ (d, $J=15.9,1$ $\mathrm{H}, \mathrm{HC}(7)), 6.24$ (dd, $J=16.1,6.6,1 \mathrm{H}, \mathrm{HC}(6)), 4.45$ (q, $J=6.0,1 \mathrm{H}, \mathrm{HC}(5))$, 3.73 (s, $\left.3 \mathrm{H}, \mathrm{H}_{3} \mathrm{C}(12)\right), 2.53$ (m, $\left.2 \mathrm{H}, \mathrm{H}_{2} \mathrm{C}(4)\right)$ ), 1.88 (s, $\left.3 \mathrm{H}, \mathrm{H}_{3} \mathrm{C}(13)\right), 1.82$ (s, $1 \mathrm{H}$ $\mathrm{OH})$

${ }^{13} \mathrm{C}$ NMR: $\quad\left(125 \mathrm{MHz}, \mathrm{CDCl}_{3}\right)$

168.37 (C(1)), 137.28 (C(3)), 136.36 (C(8)), 131.16 (C(7)), 130.99 (C(6)), 130.04 (C(9)), 128.63 (C(11)), 127.88 (C(10)), 126.55 (C(2)), 71.93 (C(5)), 51.29 (C(12)), 36.65 (C(4)), 12.75 (C(13))

IR: (neat) 3423 (m), 3025 (w), 2950 (m), 1710 (s), 1648 (m), 1494 (w), 1436 (m), 1282 (m), 1195 (m), 1128 (m), 1097 (m), 1081 (m), 1037 (m)

MS: $\quad(\mathrm{EI}, 70 \mathrm{eV})$ $245\left(\mathrm{M}^{-}, 4\right), 229$ (73), 197 (17), 169 (26), 133 (100), 115 (18)

Opt. Rot.: $\quad[\alpha]_{\mathrm{D}}^{24}-36.88(\mathrm{c}=1.0, \mathrm{EtOH})$

TLC: $\quad R_{f} 0.24$ (hexanes/EtOAc, 4/1) [UV(254)/ $\left.\mathrm{KMnO}_{4}\right]$

SFC: $\quad t_{R}(S)$-14bg, $3.78(0.4 \%) ; t_{R}(R)-14 b g, 4.62$ (99.6\%) (Chiralpak AD, 125 psi, 40 ${ }^{\circ} \mathrm{C}, 10.0 \% \mathrm{CH}_{3} \mathrm{OH}$ in $\left.\mathrm{CO}_{2}, 3.0 \mathrm{~mL} / \mathrm{min}, 220 \mathrm{~nm}\right)$ 
Analysis: $\quad \mathrm{C}_{15} \mathrm{H}_{18} \mathrm{O}_{3}(246.30)$

Calcd: $\quad$ C, $73.15 ; \quad H, 7.37 \%$

Found: $\quad$ C, $73.28 ; \quad H, 7.24 \%$

\section{Preparation of $(2 E, 5 R)$ Ethyl 5-Hydroxy-3-methyl-5-phenyl-2-pentenoate $((2 E, 5 R)-14 \mathrm{ca})$}

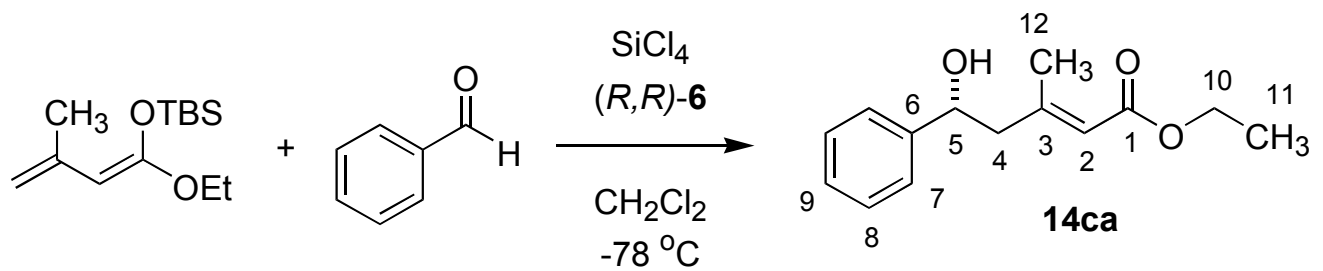

Following General Procedure 6, $8.4 \mathrm{mg}$ of bis-phosphoramide $(R, R)-6$ (0.01 mmol, 0.01 equiv) was combined with $102 \mu \mathrm{L}$ of benzaldehyde $5 \mathrm{a}(1.0 \mathrm{mmol}), 123 \mu \mathrm{L}$ of $\mathrm{SiCl}_{4}(1.1 \mathrm{mmol}, 1.1$ equiv) and $290 \mathrm{mg}$ of $\mathbf{4 c}$ (1.2 mmol, 1.2 equiv) to yield, after silica gel column chromatography (hexanes/EtOAc, 4/1), $214 \mathrm{mg}(91 \%)$ of $(2 E, 5 R)-14$ ca as a colorless oil. NMR spectral data matched that previously reported in the literature. ${ }^{25}$

Data for $(2 E, 5 R)-\mathbf{1 4 c a}$ :

마 NMR: $\quad\left(500 \mathrm{MHz}, \mathrm{CDCl}_{3}\right)$

7.37-7.26 (m, $5 \mathrm{H}, \mathrm{HC}(7,8,9)), 5.77$ (s, $1 \mathrm{H}, \mathrm{HC}(2)), 4.90(\mathrm{dd}, J=8.8,4.6,1 \mathrm{H}$, $\mathrm{HC}(5)), 4.15$ (q, $\left.J=7.0,2 \mathrm{H}, \mathrm{H}_{2} \mathrm{C}(10)\right), 2.57$ (dd, $\left.J=13.9,8.8,1 \mathrm{H}, \mathrm{HC}(4)\right), 2.51$ (dd, $J=13.7,4.4,1 \mathrm{H}, \mathrm{HC}(4)), 2.22$ (s, $\left.3 \mathrm{H}, \mathrm{H}_{3} \mathrm{C}(12)\right), 2.04$ (s, $\left.1 \mathrm{H}, \mathrm{OH}\right), 1.27$ (t, $\left.J=7.1,3 \mathrm{H}, \mathrm{H}_{3} \mathrm{C}(11)\right)$

NOE ${ }^{1} \mathrm{H}$ NMR: $\left(500 \mathrm{MHz}, \mathrm{CDCl}_{3}\right)$

Irradiation at $5.77 \mathrm{ppm}(\mathrm{HC}(2))$ enhanced signal at $2.57 \mathrm{ppm}(\mathrm{HC}(4))$ in major isomer

${ }^{13} \mathrm{C}$ NMR: $\quad\left(125 \mathrm{MHz}, \mathrm{CDCl}_{3}\right)$

166.44 (C(1)), 155.63 (C(3)), 143.69 (C(6)), 128.59 (C(8)), 127.85 (C(9)), 125.67

(C(7)), 118.55 (C(2)), 72.09 (C(5)), 59.64 (C(10)), 50.70 (C(4)), 18.94 (C(12)), $14.23(\mathrm{C}(11))$

IR: (neat)

3451 (m), 3029 (w), 2981 (m), 2940 (m), 2906 (m), 1710 (s), 1648 (s), 1452 (m), $1369(\mathrm{~m}), 1226(\mathrm{~s}), 1151(\mathrm{~s}), 1058(\mathrm{~s})$ 
MS: $\quad(E I, 70 \mathrm{eV})$

$235\left(\mathrm{M}^{+1}, 7\right), 217$ (100), 189 (7), 171 (15), 128 (80), 107 (37), 100 (27), 83 (30)

Opt. Rot.: $\quad[\alpha]_{\mathrm{D}}^{24}+19.61(\mathrm{c}=1.0, \mathrm{EtOH})$

TLC: $\quad R_{f} 0.36$ (hexanes/EtOAc, 4/1) [UV(254)/KMnO 4$]$

SFC: $\quad t_{R}(R)$-14ca, 4.04 (96.1\%); $t_{R}(S)$-14ca, 7.92 (3.9\%) (Chiralpak AD, 125 psi, 40 ${ }^{\circ} \mathrm{C}, 7.5 \% \mathrm{CH}_{3} \mathrm{OH}$ in $\left.\mathrm{CO}_{2}, 3.0 \mathrm{~mL} / \mathrm{min}, 220 \mathrm{~nm}\right)$

Analysis: $\quad \mathrm{C}_{14} \mathrm{H}_{18} \mathrm{O}_{3}(234.29)$

Calcd: $\quad$ C, $71.77 ; \quad$ H, $7.74 \%$

Found: $\quad$ C, $71.65 ; \quad \mathrm{H}, 7.98 \%$

Preparation of $(2 E, 6 E)$ Ethyl 5-Hydroxy-3-methyl-7-phenyl-2,6-heptadienoate ((2E,6E)$14 \mathrm{cg})$

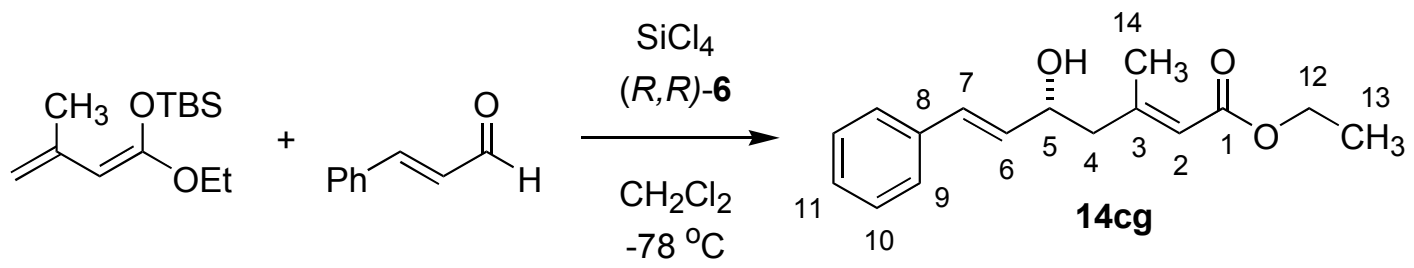

Following General Procedure 6, $8.4 \mathrm{mg}$ of bis-phosphoramide $(R, R)-6$ ( $0.01 \mathrm{mmol}, 0.01$ equiv) was combined with $126 \mu \mathrm{L}$ of cinnamaldehyde $5 \mathrm{~g}(1.0 \mathrm{mmol}), 123 \mu \mathrm{L}$ of $\mathrm{SiCl}_{4}(1.1 \mathrm{mmol}, 1.1$ equiv) and $290 \mathrm{mg}$ of $\mathbf{4 c}$ (1.2 mmol, 1.2 equiv) to yield, after silica gel column chromatography (hexanes/EtOAc, 4/1), $253 \mathrm{mg}(97 \%, 2 E / 2 Z$ 97/3) of (2E,6E)-14cg as a colorless oil.

\section{Data for $(2 E, 6 E)-\mathbf{1 4} \mathrm{cg}$ :}

${ }^{1} \mathrm{H}$ NMR: $\quad\left(500 \mathrm{MHz}, \mathrm{CDCl}_{3}\right)$

7.39-7.25 (m, $5 \mathrm{H}, \mathrm{HC}(9,10,11)), 6.63(\mathrm{~d}, J=15.8,1 \mathrm{H}, \mathrm{HC}(7)), 6.22(\mathrm{dd}, J=$ 16.0, 6.5, $1 \mathrm{H}, \mathrm{HC}(6)), 5.79$ (s, $1 \mathrm{H}, \mathrm{HC}(2)), 4.53$ (q, $J=6.7,1 \mathrm{H}, \mathrm{HC}(5)), 4.15$ (q, $\left.J=7.2,2 \mathrm{H}, \mathrm{H}_{2} \mathrm{C}(12)\right), 2.45$ (d, $\left.J=6.7,2 \mathrm{H}, \mathrm{H}_{2} \mathrm{C}(4)\right), 2.25$ (s, $\left.3 \mathrm{H}, \mathrm{H}_{3} \mathrm{C}(14)\right), 1.80$ (s, $1 \mathrm{H}, \mathrm{OH}), 1.28\left(\mathrm{t}, J=7.2,3 \mathrm{H}, \mathrm{H}_{3} \mathrm{C}(13)\right)$

NOE ${ }^{1} \mathrm{H}$ NMR: $\left(500 \mathrm{MHz}, \mathrm{CDCl}_{3}\right)$

Irradiation at $5.79 \mathrm{ppm}(\mathrm{HC}(2))$ enhanced signal at $2.45 \mathrm{ppm}(\mathrm{HC}(4))$ in major isomer 
${ }^{13} \mathrm{C}$ NMR: $\quad\left(125 \mathrm{MHz}, \mathrm{CDCl}_{3}\right)$

166.67 (C(1)), 155.57 (C(3)), 136.60 (C(8)), 131.37 (C(7)), 130.99 (C(6)), 128.85

(C(9)), 128.09 (C(11)), 126.77 (C(10)), $118.86(\mathrm{C}(2)), 70.77$ (C(5)), 59.89

$(\mathrm{C}(12)), 49.01(\mathrm{C}(4)), 19.28(\mathrm{C}(14)), 14.47(\mathrm{C}(13))$

IR: (neat)

3440 (m), 3025 (m), 2981 (m), 2939 (w), 2906 (w), 1712 (s), 1646 (m), $1494(\mathrm{w})$, $1448(\mathrm{~m}), 1382(\mathrm{~m}), 1369(\mathrm{~m}), 1295(\mathrm{~m}), 1224$ (s), 1149 (s), $1099(\mathrm{~m}), 1058(\mathrm{~m})$

MS: $\quad(E I, 70 \mathrm{eV})$

$260\left(\mathrm{M}^{+}, 1\right), 243$ (2), 213 (2), 185 (2), 157 (5), 133 (100), 115 (18), 103 (21), 91

(13), 72 (24)

Opt. Rot.: $\quad[\alpha]_{\mathrm{D}}^{24}-9.94(\mathrm{c}=1.0, \mathrm{EtOH})$

TLC: $\quad R_{f} 0.32$ (hexanes/EtOAc, 4/1) [UV(254)/KMnO 4$]$

SFC: $\quad t_{R}(R)-\mathbf{1 4 c g}, 4.10(94.0 \%) ; t_{R}(S)$-14cg, 7.35 (6.0\%) (Chiralpak AD, 125 psi, 40 ${ }^{\circ} \mathrm{C}, 12.0 \% \mathrm{CH}_{3} \mathrm{OH}$ in $\mathrm{CO}_{2}, 3.0 \mathrm{~mL} / \mathrm{min}, 220 \mathrm{~nm}$ )

Analysis: $\quad \mathrm{C}_{16} \mathrm{H}_{20} \mathrm{O}_{3}(260.33)$

Calcd: $\quad$ C, $73.82 ; \quad \mathrm{H}, 7.74 \%$

Found: $\quad$ C, $73.55 ; \quad \mathrm{H}, 7.83 \%$

\section{Preparation of (2E) Ethyl 5-Hydroxy-3-methyl-7-phenyl-2-heptenoate ((2E)-14ck)}

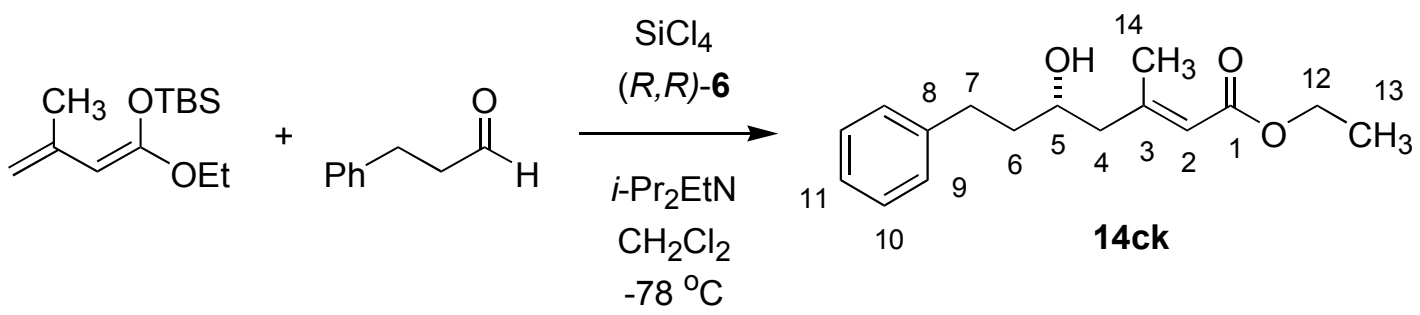

Following General Procedure 7, $42 \mathrm{mg}$ of bis-phosphoramide $(R, R)-6$ ( $0.05 \mathrm{mmol}, 0.05$ equiv) was combined with $8 \mu \mathrm{L}$ of $i-\operatorname{Pr}_{2} \operatorname{EtN}(0.05 \mathrm{mmol}, 0.05$ equiv), $126 \mu \mathrm{L}$ of hydrocinnamaldehyde $5 \mathbf{k}(1.0 \mathrm{mmol}), 123 \mu \mathrm{L}$ of $\mathrm{SiCl}_{4}$ (1.1 mmol, 1.1 equiv) and $290 \mathrm{mg}$ of $\mathbf{4 c}(1.2 \mathrm{mmol}, 1.2$ equiv) to yield, after silica gel column chromatography (hexanes/EtOAc, 9/1), $190 \mathrm{mg}(73 \%)$ of $(2 E)$ 14ck as a colorless oil. 


\section{Data for $(2 E)-\mathbf{1 4} \mathbf{c k}:$}

${ }^{1} \mathrm{H}$ NMR: $\quad\left(500 \mathrm{MHz}, \mathrm{CDCl}_{3}\right)$

7.31-7.18 (m, $5 \mathrm{H}, \mathrm{HC}(9,10,11)), 5.74$ (s, $1 \mathrm{H}, \mathrm{HC}(2)), 4.15$ (q, $J=7.2,2 \mathrm{H}$ $\mathrm{H}_{2} \mathrm{C}(12)$ ), 3.84 (pent, $J=6.5,1 \mathrm{H}, \mathrm{HC}(5)$ ), 2.83 (dt, $J=13.7,7.9,1 \mathrm{H}, \mathrm{HC}(7)$ ), 2.70 (dt, $J=13.8,8.3,1 \mathrm{H}, \mathrm{HC}(7)$ ), 2.33 (dd, $J=13.5,4.0,1 \mathrm{H}, \mathrm{HC}(4)), 2.27$ (dd, $J=13.5,8.6,1 \mathrm{H}, \mathrm{HC}(4)), 2.17$ (s, $\left.3 \mathrm{H}, \mathrm{H}_{3} \mathrm{C}(14)\right), 1.79$ (q, $\left.J=7.72,2 \mathrm{H}, \mathrm{H}_{2} \mathrm{C}(6)\right)$, 1.65 (s, $1 \mathrm{H}, \mathrm{OH}), 1.28$ (t, $\left.J=7.1,3 \mathrm{H}, \mathrm{H}_{3} \mathrm{C}(13)\right)$

NOE ${ }^{1} \mathrm{H}$ NMR: $\left(500 \mathrm{MHz}, \mathrm{CDCl}_{3}\right)$

Irradiation at $5.74 \mathrm{ppm}(\mathrm{HC}(2))$ enhanced signal at $2.27 \mathrm{ppm}(\mathrm{HC}(4))$ in major isomer

${ }^{13} \mathrm{C} \mathrm{NMR}: \quad\left(125 \mathrm{MHz}, \mathrm{CDCl}_{3}\right)$

$166.41(\mathrm{C}(1)), 156.06(\mathrm{C}(3)), 141.69(\mathrm{C}(8)), 128.46(\mathrm{C}(9)), 128.40(\mathrm{C}(10))$, 125.95 (C(11)), 118.32 (C(2)), 68.62 (C(5)), 59.65 (C(12)), 49.04 (C(4)), 38.86 $(\mathrm{C}(7)), 31.96(\mathrm{C}(6)), 18.92(\mathrm{C}(14)), 14.23(\mathrm{C}(13))$

IR: (neat)

$3450(\mathrm{~m}), 3025(\mathrm{w}), 2981(\mathrm{~m}), 2939(\mathrm{~m}), 2863(\mathrm{~m}), 1712(\mathrm{~s}), 1644(\mathrm{~s}), 1496(\mathrm{~m})$, $1454(\mathrm{~m}), 1369(\mathrm{~m}), 1280(\mathrm{~m}), 1226(\mathrm{~s}), 1149(\mathrm{~s}), 1068(\mathrm{~m})$

MS: $\quad(\mathrm{EI}, 70 \mathrm{eV})$

$263\left(\mathrm{M}^{+1}, 1\right), 244$ (8), 217 (16), 171 (3), 128 (100), 117 (13), 100 (30), 91 (48), $82(21)$

Opt. Rot.: $\quad[\alpha]_{\mathrm{D}}^{24}-5.85(\mathrm{c}=1.0, \mathrm{EtOH})$

TLC: $\quad R_{f} 0.51$ (hexanes/EtOAc, $\left.4 / 1\right)\left[\mathrm{UV}(254) / \mathrm{KMnO}_{4}\right]$

SFC: $\quad t_{R}(S)$-14ck, $3.52(97.5 \%) ; t_{R}(R)$-14ck, 4.18 (2.5\%) (Chiralpak AD, 125 psi, 40 ${ }^{\circ} \mathrm{C}, 12.0 \% \mathrm{CH}_{3} \mathrm{OH}$ in $\left.\mathrm{CO}_{2}, 3.0 \mathrm{~mL} / \mathrm{min}, 220 \mathrm{~nm}\right)$

Analysis: $\quad \mathrm{C}_{16} \mathrm{H}_{22} \mathrm{O}_{3}(262.34)$

Calcd: $\quad$ C, $73.25 ; \quad \mathrm{H}, 8.45 \%$

Found: $\quad$ C, $73.16 ; \quad \mathrm{H}, 8.22 \%$ 


\section{Preparation of $(2 E, 4 R, 5 R)$ tert-Butyl 5-Hydroxy-4-methyl-5-phenyl-2-pentenoate $((2 E, 4 R, 5 R)-14 d a)$}

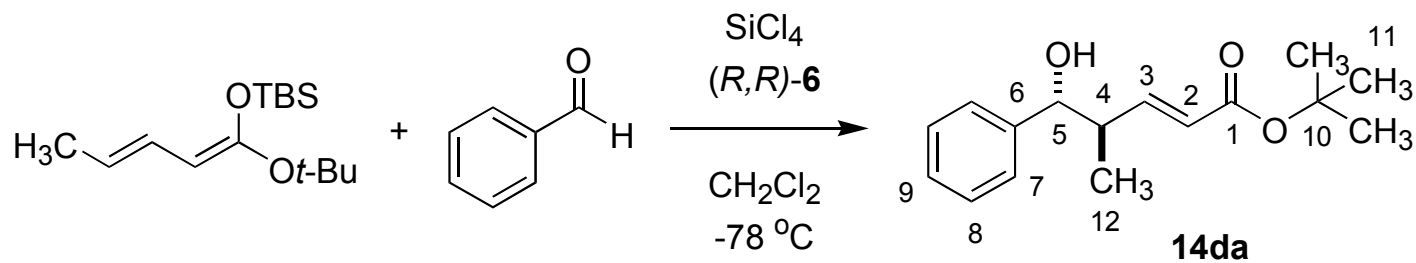

Following General Procedure 6, $8.4 \mathrm{mg}$ of bis-phosphoramide $(R, R)-6$ (0.01 mmol, 0.01 equiv) was combined with $102 \mu \mathrm{L}$ of benzaldehyde $5 \mathbf{a}(1.0 \mathrm{mmol}), 123 \mu \mathrm{L}$ of $\mathrm{SiCl}_{4}(1.1 \mathrm{mmol}, 1.1$ equiv) and $324 \mathrm{mg}$ of $\mathbf{4 d}$ (1.2 mmol, 1.2 equiv) to yield, after silica gel column chromatography (hexanes/EtOAc, 4/1), $242 \mathrm{mg}(92 \%)$ of $(2 E, 4 R, 5 R)-14 \mathrm{da}$ as a colorless oil.

Data for $(2 E, 4 R, 5 R)-14 \mathrm{da}$ :

1 NMR: $\quad\left(400 \mathrm{MHz}, \mathrm{CDCl}_{3}\right)$

7.37-7.29 (m, 5 H, HC(7,8,9)), 6.91 (dd, $J=15.6,8.3,1 \mathrm{H}, \mathrm{HC}(3)), 5.84$ (dd, $J=$ 15.9, 0.7, 1 H, HC(2)), 4.47 (d, $J=7.6,1$ H, HC(5)), 2.62 (m, 1 H, HC(4)), 2.65

(s, $1 \mathrm{H}, \mathrm{OH}), 1.48$ (s, $\left.9 \mathrm{H}, \mathrm{H}_{3} \mathrm{C}(11)\right), 0.89$ (d, $\left.J=6.8,3 \mathrm{H}, \mathrm{H}_{3} \mathrm{C}(12)\right)$

${ }^{13} \mathrm{C} \mathrm{NMR}: \quad\left(100 \mathrm{MHz}, \mathrm{CDCl}_{3}\right)$

$165.74(\mathrm{C}(1)), 149.76(\mathrm{C}(3)), 142.17(\mathrm{C}(6)), 128.45$ (C(8)), 127.95 (C(9)), 126.66 (C(7)), $124.11(\mathrm{C}(2)), 80.27$ (C(10)), 77.94 (C(5)), 44.28 (C(4)), 28.12 (C(11)), $16.17(\mathrm{C}(12))$

IR: (neat)

3442 (m), 2977 (s), 2933 (m), 1710 (s), 1650 (m), 1454 (m), 1367 (m), 1313 (m), $1253(\mathrm{~m}), 1157(\mathrm{~s}), 1035(\mathrm{~m})$

MS: $\quad(E I, 70 \mathrm{eV})$

$263\left(\mathrm{M}^{+}, 29\right), 189$ (100), 143 (37), 100(39)

Opt. Rot.: $\quad[\alpha]_{\mathrm{D}}^{24}+65.37(\mathrm{c}=1.0, \mathrm{EtOH})$

TLC: $\quad R_{f} 0.45$ (hexanes/EtOAc, 4/1) [UV(254)/ $\left.\mathrm{KMnO}_{4}\right]$

SFC: $\quad t_{R}(4 R, 5 R)-14 d a, 6.20(94.5 \%) ; t_{R}(4 S, 5 S)-14 d a, 7.05$ (5.5\%) (Chiralpak AD, 125 psi, $40{ }^{\circ} \mathrm{C}, 3.0 \% \mathrm{CH}_{3} \mathrm{OH}$ in $\mathrm{CO}_{2}, 3.0 \mathrm{~mL} / \mathrm{min}, 220 \mathrm{~nm}$ ) 
Analysis: $\quad \mathrm{C}_{16} \mathrm{H}_{22} \mathrm{O}_{3}(262.16)$

Calcd: $\quad$ C, $73.25 ; \quad H, 8.45 \%$

Found: $\quad$ C, $73.18 ; \quad$ H, 8.74\%

\section{Preparation of $(2 E, 6 E)$ tert-Butyl 5-Hydroxy-4-methyl-7-phenyl-2,6-heptadienoate $((2 E, 6 E)-14 d g)$}

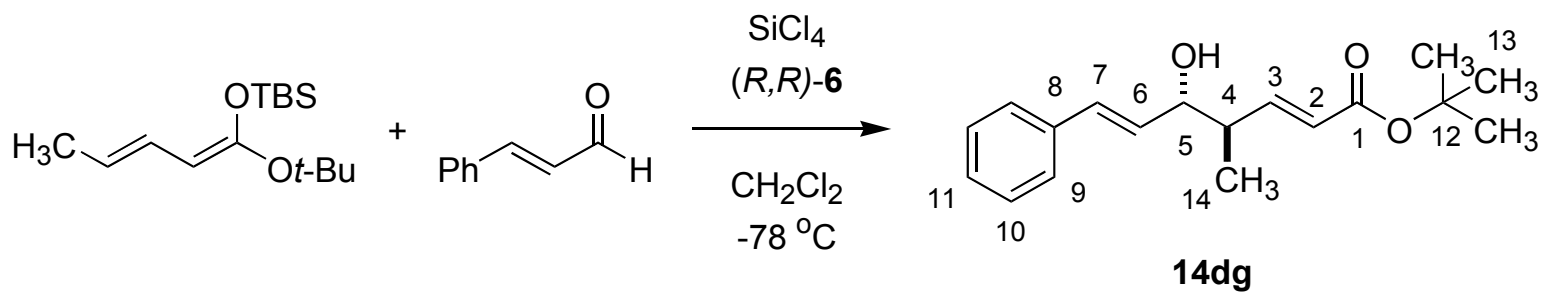

Following General Procedure 6, $8.4 \mathrm{mg}$ of bis-phosphoramide $(R, R)-6$ (0.01 mmol, 0.01 equiv) was combined with $126 \mu \mathrm{L}$ of cinnamaldehyde $5 \mathrm{~g}(1.0 \mathrm{mmol}), 123 \mu \mathrm{L}$ of $\mathrm{SiCl}_{4}(1.1 \mathrm{mmol}, 1.1$ equiv) and $324 \mathrm{mg}$ of $\mathbf{4 d}$ (1.2 mmol, 1.2 equiv) to yield, after silica gel column chromatography (hexanes/EtOAc, 9/1), $205 \mathrm{mg}(71 \%)$ of $(2 E, 6 E)-\mathbf{1 4 d g}$ as a colorless oil.

Data for $(2 E, 6 E)-\mathbf{1 4 d g}$ :

mp: $\quad 62-66^{\circ} \mathrm{C}$

${ }^{1} \mathrm{H}$ NMR: $\quad\left(400 \mathrm{MHz}, \mathrm{CDCl}_{3}\right)$

7.39-7.23 (m, 5 H, HC(9,10,11)), 6.90 (dd, $J=16.1,8.0,1 \mathrm{H}, \mathrm{HC}(3)), 6.61$ (d, $J=$ 15.9, $1 \mathrm{H}, \mathrm{HC}(7)), 6.19(\mathrm{dd}, J=15.9,7.1,1 \mathrm{H}, \mathrm{HC}(6)), 5.85(\mathrm{dd}, J=15.6,1.0,1$ H, HC(2)), 4.16 (t, J=6.8, 1 H, HC(5)), 2.52 (m, 1 H, HC(4)), 1.78 (s, $1 \mathrm{H}, \mathrm{OH})$, 1.48 (s, $\left.9 \mathrm{H}, \mathrm{H}_{3} \mathrm{C}(13)\right), 1.09$ (d, $\left.J=6.8,3 \mathrm{H}, \mathrm{H}_{3} \mathrm{C}(14)\right)$

${ }^{13} \mathrm{C} \mathrm{NMR}: \quad\left(100 \mathrm{MHz}, \mathrm{CDCl}_{3}\right)$

165.81 (C(1)), 148.75 (C(3)), 136.28 (C(8)), 132.36 (C(7)), 129.65 (C(6)), 128.58 (C(9)), 127.68 (C(11)), 126.59 (C(10)), 124.14 (C(2)), 80.33 (C(12)), 76.17 (C(5)), 42.91 (C(4)), 28.12 (C(13)), 15.66 (C(14))

IR: $\quad\left(\mathrm{CHCl}_{3}\right)$

3605 (w), 3027 (m), 3013 (m), 2981 (m), 1705 (s), 1651 (m), 1494 (m), 1455 (m), $1369(\mathrm{~m}), 1301(\mathrm{~m}), 1158(\mathrm{~s})$

MS: $\quad(\mathrm{CI}, 70 \mathrm{eV})$ $289\left(\mathrm{M}^{+1}, 1\right), 271$ (33), 215 (100), 197 (9), 169 (10), 133 (21), 100 (3) 
Opt. Rot.: $\quad[\alpha]_{\mathrm{D}}^{24}+5.78(\mathrm{c}=1.0, \mathrm{EtOH})$

TLC: $\quad R_{f} 0.58$ (hexanes/EtOAc, 4/1) [UV(254)/ $\left.\mathrm{KMnO}_{4}\right]$

SFC: $\quad t_{R}(4 S, 5 R)-\mathbf{1 4 d g}, 8.29(8.8 \%) ; t_{R}(4 R, 5 S)-14 d g, 9.15$ (91.2\%) (Chiralpak OJ, 125 psi, $40{ }^{\circ} \mathrm{C}, 4.0 \% \mathrm{CH}_{3} \mathrm{OH}$ in $\mathrm{CO}_{2}, 2.0 \mathrm{~mL} / \mathrm{min}, 220 \mathrm{~nm}$ )

Analysis: $\quad \mathrm{C}_{18} \mathrm{H}_{24} \mathrm{O}_{3}(288.38)$

Calcd: $\quad$ C, $74.97 ; \quad H, 8.39 \%$

Found: $\quad$ C, $75.11 ; \quad H, 8.59 \%$

\section{Preparation of (5R)-6-(2-Hydroxy-2-phenylethyl)-2,2-dimethyl-[1,3]-dioxin-4-one ((5R)-}

15a)

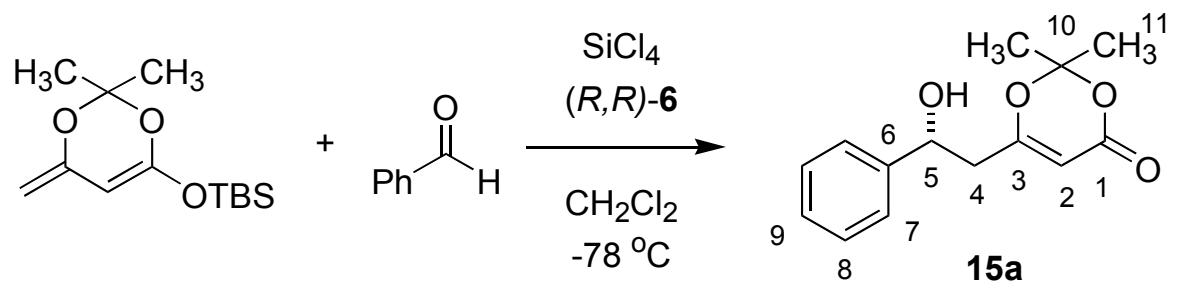

Following General Procedure 6, $8.4 \mathrm{mg}$ of bis-phosphoramide $(R, R)-6$ (0.01 mmol, 0.01 equiv) was combined with $102 \mu \mathrm{L}$ of benzaldehyde 5a $(1.0 \mathrm{mmol}), 123 \mu \mathrm{L}$ of $\mathrm{SiCl}_{4}(1.1 \mathrm{mmol}, 1.1$ equiv) and $307 \mathrm{mg}$ of $\mathbf{1 3}$ (1.2 mmol, 1.2 equiv) to yield, after silica gel column chromatography (hexanes/EtOAc, 6/4), $228 \mathrm{mg}(92 \%)$ of (5R)-15a as a white, crystalline solid. NMR spectral data matched that previously reported in the literature. ${ }^{26}$

Data for $(5 R)-\mathbf{1 5 a}$ :

mp: $\quad 70-72{ }^{\circ} \mathrm{C}$

1 NMR: $\quad\left(400 \mathrm{MHz}, \mathrm{CDCl}_{3}\right)$

7.4-7.28 (m, 5 H, HC(7,8,9)), 5.27 (s, 1 H, HC(2)), 4.98 (m, 1 H, HC(5)), 2.7 (dd, $J=14.6,8.2,1 \mathrm{H}, \mathrm{HC}(4)), 2.55(\mathrm{dd}, J=14.6,4.8,1 \mathrm{H}, \mathrm{HC}(4)), 2.1$ (d, $J=4.0,1$ $\mathrm{H}, \mathrm{OH}), 1.65$ (s, $\left.3 \mathrm{H}, \mathrm{H}_{3} \mathrm{C}(11)\right), 1.63$ (s, $\left.3 \mathrm{H}, \mathrm{H}_{3} \mathrm{C}(11)\right)$

${ }^{13} \mathrm{C}$ NMR: $\quad\left(100 \mathrm{MHz}, \mathrm{CDCl}_{3}\right)$

168.19 (C(3)), 161.2 (C(1)), 142.65 (C(6)), 128.74 (C(8)), 128.33 (C(9)), 125.69 (C(7)), $106.66(\mathrm{C}(10)), 95.38$ (C(2)), 71.22 (C(5)), 43.16 (C(4)), 25.39 (C(11)), $24.70(\mathrm{C}(11))$ 
MS: $\quad(\mathrm{CI}$, methane, $70 \mathrm{eV})$

$249\left(\mathrm{M}^{+1}, 29\right), 191$ (28), 173 (32), 147 (22), 131 (100), 105 (25), 85 (21)

Opt. Rot.: $\quad[\alpha]_{\mathrm{D}}^{24}+35.9\left(\mathrm{c}=1.0, \mathrm{CHCl}_{3}\right)$, lit. $[\alpha]_{\mathrm{D}}^{24}+35.1\left(\mathrm{c}=1.0, \mathrm{CHCl}_{3}\right)$ for $(5 R)-\mathbf{X X}^{26}$

TLC: $\quad R_{f} 0.35$ (hexanes/EtOAc, 6/4) [UV(254)/KMnO $\mathrm{KM}_{4}$

SFC: $\quad t_{R}(S)-15 a, 3.29(12.8 \%) ; t_{R}(R)-15 a, 3.53(87.2 \%)$ (Chiralpak AD, 125 psi, $40{ }^{\circ} \mathrm{C}$, $10.0 \% \mathrm{CH}_{3} \mathrm{OH}$ in $\mathrm{CO}_{2}, 3.0 \mathrm{~mL} / \mathrm{min}, 220 \mathrm{~nm}$ )

\section{Preparation of (5R)-6-(2-Hydroxy-4-phenyl 3-butenyl)-2,2-dimethyl-[1,3]-dioxin-4-one ((5R)-15g)}

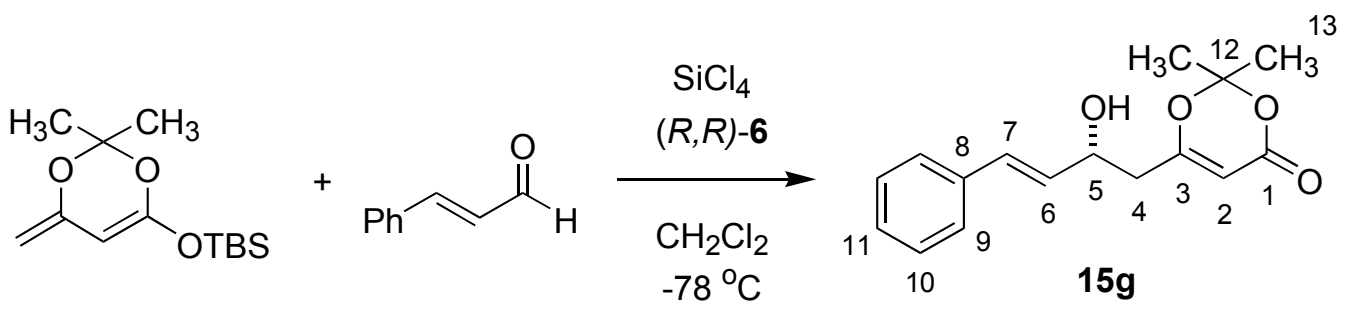

Following General Procedure 6, $8.4 \mathrm{mg}$ of bis-phosphoramide $(R, R)-6$ (0.01 mmol, 0.01 equiv) was combined with $126 \mu \mathrm{L}$ of cinnamaldehyde $5 \mathrm{~g}(1.0 \mathrm{mmol}), 123 \mu \mathrm{L}$ of $\mathrm{SiCl}_{4}(1.1 \mathrm{mmol}, 1.1$ equiv) and $290 \mathrm{mg}$ of $\mathbf{1 3}$ (1.2 mmol, 1.2 equiv) to yield, after silica gel column chromatography (hexanes/EtOAc, 6/4), $241 \mathrm{mg}(88 \%)$ of (5R)-15g as a white, crystalline solid. NMR spectral data matched that previously reported in the literature. ${ }^{26}$

Data for $(5 R)-15 g$ :

mp: $\quad 81-82^{\circ} \mathrm{C}$

${ }^{1} \mathrm{H}$ NMR: $\quad\left(400 \mathrm{MHz}, \mathrm{CDCl}_{3}\right)$

7.37-7.25 (m, $5 \mathrm{H}, \mathrm{HC}(9,10,11)), 6.63(\mathrm{~d}, J=16.1,1 \mathrm{H}, \mathrm{HC}(7)), 6.21(\mathrm{dd}, J=$ 15.8, 6.9, $1 \mathrm{H}, \mathrm{HC}(6)), 5.36$ (s, $1 \mathrm{H}, \mathrm{HC}(2)), 4.6$ (q, $J=6.9,1 \mathrm{H}, \mathrm{HC}(5)), 2.55$ (m, $\left.2 \mathrm{H}, \mathrm{H}_{2} \mathrm{C}(4)\right), 1.95$ (s, $\left.1 \mathrm{H}, \mathrm{OH}\right), 1.69$ (s, $\left.3 \mathrm{H}, \mathrm{H}_{3} \mathrm{C}(13)\right), 1.68$ (s, $\left.3 \mathrm{H}, \mathrm{H}_{3} \mathrm{C}(13)\right)$

${ }^{13} \mathrm{C} \mathrm{NMR}: \quad\left(100 \mathrm{MHz}, \mathrm{CDCl}_{3}\right)$

168.19 (C(3)), 161.03 (C(1)), 135.96 (C(8)), 131.62 (C(7)), 130.13 (C(6)), 128.72 (C(9)), 128.17 (C(11)), 126.55 (C(10)), 106.72 (C(12)), 95.43 (C(2)), 69.83 (C(5)), 41.50 (C(4)), 25.34 (C(13)), 24.84 (C(13)) 
MS: $\quad(\mathrm{EI}, 70 \mathrm{eV})$

$275\left(\mathrm{M}^{+1}, 2\right), 216(45), 188$ (60), 172 (23), 157 (18), 131 (44), 115 (32), 104 (100), 84 (41)

Opt. Rot.: $\quad[\alpha]_{\mathrm{D}}^{24}+14.43(\mathrm{c}=1.0, \mathrm{EtOH}),[\alpha]_{\mathrm{D}}^{24}+6.79\left(\mathrm{c}=1.0, \mathrm{CHCl}_{3}\right)$, lit $[\alpha]_{\mathrm{D}}^{24}+6.2(\mathrm{c}=1.0$, $\left.\mathrm{CHCl}_{3}\right)$ for $(5 R)-\mathbf{1 5} \mathbf{g}^{26}$

TLC: $\quad R_{f} 0.28$ (hexanes/EtOAc, 6/4) [UV(254)/ $\left.\mathrm{KMnO}_{4}\right]$

SFC: $\quad t_{R}(S)-15 g, 6.56(11.1 \%) ; t_{R}(R)-15 g, 7.9(88.9 \%)$ (Chiralpak AD, 125 psi, $40{ }^{\circ} \mathrm{C}$, $8.0 \% \mathrm{CH}_{3} \mathrm{OH}$ in $\mathrm{CO}_{2}, 3.0 \mathrm{~mL} / \mathrm{min}, 220 \mathrm{~nm}$ )

\section{Preparation of (5S)-6-(2-Hydroxy-4-phenylbutyl)-2,2-dimethyl-[1,3]-dioxin-4-one ((5S)-} $15 \mathrm{k})$

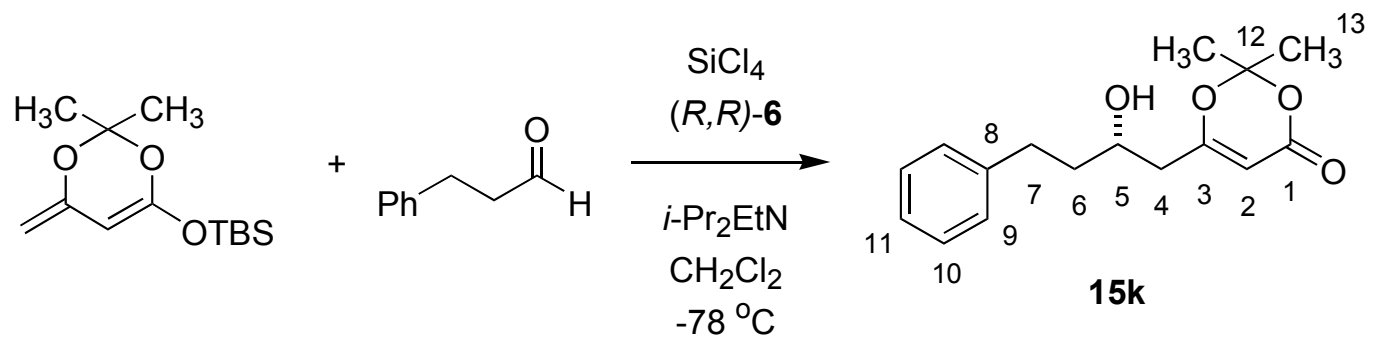

Following General Procedure 7, $42 \mathrm{mg}$ of bis-phosphoramide $(R, R)-6$ (0.05 mmol, 0.05 equiv) was combined with $8 \mu \mathrm{L}$ of $i-\operatorname{Pr}_{2} \operatorname{EtN}(0.05 \mathrm{mmol}, 0.05$ equiv), $126 \mu \mathrm{L}$ of hydrocinnamaldehyde 5k (1.0 mmol), $123 \mu \mathrm{L}$ of $\mathrm{SiCl}_{4}(1.1 \mathrm{mmol}, 1.1$ equiv) and $272 \mathrm{mg}$ of $\mathbf{1 3}$ (1.2 mmol, 1.2 equiv) to yield, after silica gel column chromatography (hexanes/EtOAc, 6/4), $230 \mathrm{mg}$ (83 \%) of (5S)-15k as a white, crystalline solid. NMR spectral data matched that previously reported in the literature. $^{26}$

Data for $(5 S)-\mathbf{1 5 k}$ :

mp: $\quad 59-60{ }^{\circ} \mathrm{C}$

1 NMR: $\quad\left(500 \mathrm{MHz}, \mathrm{CDCl}_{3}\right)$

7.3-7.18 (m, $5 \mathrm{H}, \mathrm{HC}(9,10,11)), 5.31$ (s, $1 \mathrm{H}, \mathrm{HC}(2)), 3.92$ (pent, $J=6.2,1 \mathrm{H}$, HC(5)), 2.81 (m, $1 \mathrm{H}, \mathrm{HC}(4)), 2.70$ (m, $1 \mathrm{H}, \mathrm{HC}(4)), 2.42$ (dd, J=14.4, 4.3, $1 \mathrm{H}$, HC(6)), 2.36 (dd, $J=14.6,8.1,1 \mathrm{H}, \mathrm{HC}(6)), 1.82$ (m, 2 H, $\left.\mathrm{H}_{2} \mathrm{C}(7)\right), 1.68$ (s, $3 \mathrm{H}$, $\left.\mathrm{H}_{3} \mathrm{C}(12)\right), 1.66$ (s, $\left.3 \mathrm{H}, \mathrm{H}_{3} \mathrm{C}(12)\right)$ 
${ }^{13} \mathrm{C}$ NMR: $\quad\left(125 \mathrm{MHz}, \mathrm{CDCl}_{3}\right)$

168.95 (C(3)), 161.0 (C(1)), 141.27 (C(8)), 128.55 (C(9)), 128.37 (C(10)), 126.11

(C(11)), 106.63 (C(12)), 95.14 (C(2)), 68.32 (C(5)), 41.73 (C(4)), 38.89 (C(7)), $31.77(\mathrm{C}(6)), 25.16(\mathrm{C}(13)), 24.84(\mathrm{C}(13))$

MS: $\quad(E I, 70 \mathrm{eV})$

$277\left(\mathrm{M}^{+}, 3\right), 218$ (28), 200 (31), 172 (7), 158 (16), 130 (24), 117 (44), 104 (22), $91(100), 69(26)$

Opt. Rot.: $\quad[\alpha]_{\mathrm{D}}^{24}+10.17(\mathrm{c}=1.0, \mathrm{EtOH}),[\alpha]_{\mathrm{D}}^{24}+3.4\left(\mathrm{c}=1.0, \mathrm{CHCl}_{3}\right)$, lit. $[\alpha]_{\mathrm{D}}^{24}+2.5(\mathrm{c}=1.0$, $\left.\mathrm{CHCl}_{3}\right)$ for $(5 S)-\mathbf{1 5} \mathbf{k}^{26}$

TLC: $\quad R_{f} 0.27$ (hexanes/EtOAc, 6/4) [UV(254)/ $\left.\mathrm{KMnO}_{4}\right]$

SFC: $\quad t_{R}(R)-15 k$, $4.80(5.4 \%) ; t_{R}(S)-15 k, 5.18$ (94.6\%) (Chiralpak AD, 125 psi, $40{ }^{\circ} \mathrm{C}$, $8.0 \% \mathrm{CH}_{3} \mathrm{OH}$ in $\mathrm{CO}_{2}, 3.0 \mathrm{~mL} / \mathrm{min}, 220 \mathrm{~nm}$ )

\section{General Procedure 8. Ozonolyis of an $\alpha, \beta$-Unsaturated Ester. Preparation of (3R)-1-}

\section{Phenyl propane-1,3-diol ((3R)-16) from (2E,5R) Ethyl 5-Hydroxy-5-phenyl-2-pentenoate} $((2 E, 5 R)-14 a a)$

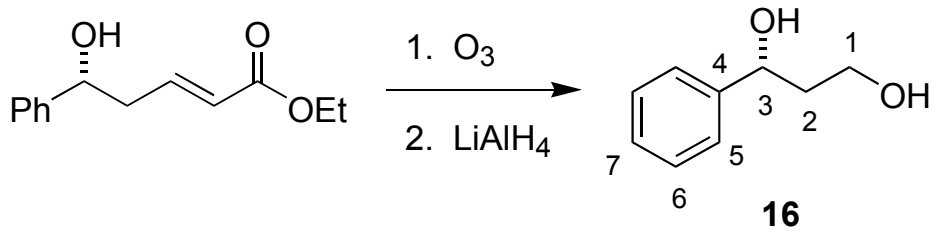

A $25 \mathrm{~mL}$, 1-neck, round-bottom flask containing a solution of $94 \mathrm{mg}(2 E, 5 R)$ ethyl 5-hydroxy-5phenyl-2-pentenoate $\left((2 E, 5 R)\right.$-14aa) $(0.42 \mathrm{mmol})$ in $5 \mathrm{~mL}$ of pentane was cooled to $-78{ }^{\circ} \mathrm{C}$ in a dry ice/isopropanol bath and a solution of ozone in oxygen was passed through it using a gas dispersion tube for $1 \mathrm{~h}$. The solution was sparged with $\mathrm{N}_{2}$ (to remove residual ozone) and then $2.89 \mathrm{~mL}$ of a solution of $\mathrm{LiAlH}_{4}$ in THF $(0.58 \mathrm{M}, 1.68 \mathrm{mmol}, 4$ equiv $)$ was added slowly at -78 ${ }^{\circ} \mathrm{C}$ in a dry ice/isopropanol bath. The flask was then fitted with a reflux condenser and was heated at $65{ }^{\circ} \mathrm{C}$ for $1 \mathrm{~h}$. The mixture was cooled to room temperature and $5 \mathrm{~mL}$ of sat. aq. sodium potassium tartrate solution was added and the mixture was stirred to break up the resulting precipitate. The organic layer was separated and the aqueous layer was washed with $\mathrm{Et}_{2} \mathrm{O}(3 \times 10$ $\mathrm{mL})$. The combined organic extracts were washed with brine $(25 \mathrm{~mL})$, dried over $\mathrm{Na}_{2} \mathrm{SO}_{4}(5 \mathrm{~g})$, filtered and the filtrate was concentrated in vacuo. The residue was purified by silica gel column 
chromatography (EtOAc/ hexanes, 2/1) to yield $41 \mathrm{mg}(64 \%)$ of $(3 R)-\mathbf{1 6}$ as a colorless oil. NMR spectral data matched that previously reported in the literature. ${ }^{27}$

Data for $(3 R)-\mathbf{1 6}$ :

${ }^{1} \mathrm{H}$ NMR: $\quad\left(400 \mathrm{MHz}, \mathrm{CDCl}_{3}\right)$

7.36-7.25 (m, $5 \mathrm{H}, \mathrm{HC}(5,6,7)), 4.96$ (dd, $J=8.5,3.9,1 \mathrm{H}, \mathrm{HC}(3)), 3.86$ (t, $J=5.6$,

$\left.2 \mathrm{H}, \mathrm{H}_{2} \mathrm{C}(1)\right), 2.55$ (s, $\left.1 \mathrm{H}, \mathrm{OH}\right), 2.1-1.85$ (m, $\left.2 \mathrm{H}, \mathrm{H}_{2} \mathrm{C}(2)\right)$

Opt. Rot.: $\quad[\alpha]_{\mathrm{D}}^{24}+60.8\left(\mathrm{c}=1.0, \mathrm{CHCl}_{3}\right)$, lit. $[\alpha]_{\mathrm{D}}^{24}-63.0\left(\mathrm{c}=0.958, \mathrm{CHCl}_{3}\right)$ for $(3 S)-\mathbf{1 6}^{27}$

TLC: $\quad R_{f} 0.28$ (EtOAc/hexanes, 2/1) [p-anisaldehyde]

\section{Preparation of (5R) Ethyl 5-Hydroxy-7-phenylheptanoate ((5R)-17) from $(2 E, 5 R, 6 E)$ Ethyl} 5-Hydroxy-7-phenyl-2,6-heptadienoate $((2 E, 5 R, 6 E)-14 a g)$

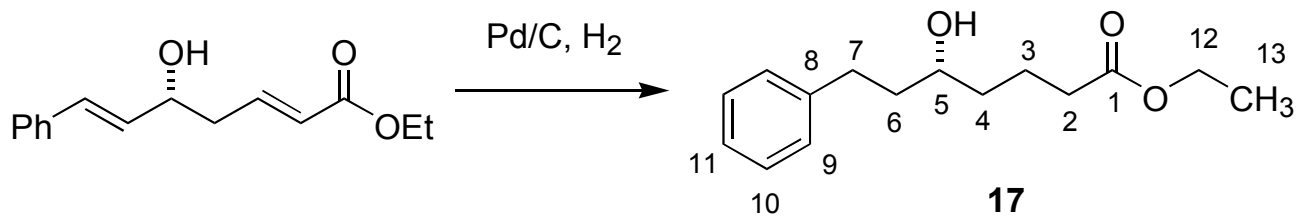

A $50 \mathrm{~mL}, 1$-neck, round-bottom flask containing a suspension of $246 \mathrm{mg}$ of $(2 E, 5 R, 6 E)$ ethyl 5hydroxy-7-phenyl-2,6-heptadienoate $((2 E, 5 R, 6 E)-14 a g)(1.0 \mathrm{mmol})$ and $7.0 \mathrm{mg}$ of $10 \% \mathrm{Pd}-\mathrm{C}$ in $25 \mathrm{~mL}$ of EtOAc was stirred for $3 \mathrm{~h}$ at $23{ }^{\circ} \mathrm{C}$ under $1 \mathrm{~atm}$ of $\mathrm{H}_{2}$. After briefly evacuating the flask and venting with $\mathrm{N}_{2}$, the suspension was then filtered through Celite and the filtrate was concentrated in vacuo. The residue was purified by silica gel column chromatography (hexanes/EtOAc, 4/1) to yield $206 \mathrm{mg}(82 \%)$ of $(5 R)-17$ as a colorless oil.

Data for $(5 R)-17$ :

1 NMR: $\quad\left(400 \mathrm{MHz}, \mathrm{CDCl}_{3}\right)$

7.30-7.16 (m, $5 \mathrm{H}, \mathrm{HC}(9,10,11)), 4.12\left(\mathrm{q}, J=7.1,2 \mathrm{H}, \mathrm{H}_{2} \mathrm{C}(12)\right), 3.61(\mathrm{~m}, 1 \mathrm{H}$, $\mathrm{HC}(5)), 2.77$ (m, $1 \mathrm{H}, \mathrm{HC}(7)), 2.68$ (m, $1 \mathrm{H}, \mathrm{HC}(7)), 2.32$ (dt, $J=1.2,7.56,2 \mathrm{H}$, $\left.\mathrm{H}_{2} \mathrm{C}(2)\right)$, 1.79-1.67 (m, $4 \mathrm{H}, 2$ x $\left.\mathrm{H}_{2} \mathrm{C}(4,6)\right), 1.50$ (m, $\left.2 \mathrm{H}, \mathrm{H}_{2} \mathrm{C}(3)\right)$, 1.24 (t, $J=7.1$, $\left.3 \mathrm{H}, \mathrm{H}_{3} \mathrm{C}(13)\right)$

${ }^{13} \mathrm{C}$ NMR: $\quad\left(100 \mathrm{MHz}, \mathrm{CDCl}_{3}\right)$

$173.78(\mathrm{C}(1)), 142.01(\mathrm{C}(8)), 128.37(\mathrm{C}(9)), 128.35(\mathrm{C}(10)), 125.29$ (C(11)), $70.65(\mathrm{C}(5)), 60.34(\mathrm{C}(12)), 39.63(\mathrm{C}(7)), 36.83(\mathrm{C}(4)), 34.05(\mathrm{C}(2)), 31.99$ $(C(6)), 20.84(C(3)), 14.21(C(13))$ 
IR: (neat)

$3451(\mathrm{w}), 2980(\mathrm{w}), 2866(\mathrm{w}), 1733$ (s), 1495 (w), 1454 (m), 1373 (m), 1238 (m), $1180(\mathrm{~m}), 1094(\mathrm{~m}), 1031(\mathrm{~m})$

MS: $\quad(\mathrm{EI}, 70 \mathrm{eV})$

$251\left(\mathrm{M}^{+1}, 1\right), 232$ (35), 204 (7), 186 (70), 117 (35), 104 (40), 91 (100), 71 (19)

Opt. Rot.: $\quad[\alpha]_{\mathrm{D}}^{24}-5.94(\mathrm{c}=1.0, \mathrm{EtOH})$

TLC: $\quad R_{f} 0.19$ (hexanes/EtOAc, 4/1) [UV(254)/KMnO 4

Analysis: $\quad \mathrm{C}_{15} \mathrm{H}_{22} \mathrm{O}_{3}(250.33)$

Calcd: $\quad$ C, $71.97 ; \quad H, 8.86 \%$

Found: $\quad$ C, $71.91 ; \quad H, 8.91 \%$

\section{Preparation of (5R) Ethyl 5-Hydroxy-7-phenylheptanoate (5R)-17 from (2E,5S) Ethyl 5-} Hydroxy-7-phenyl-2-heptenoate $(2 E, 5 S)-14$ ak

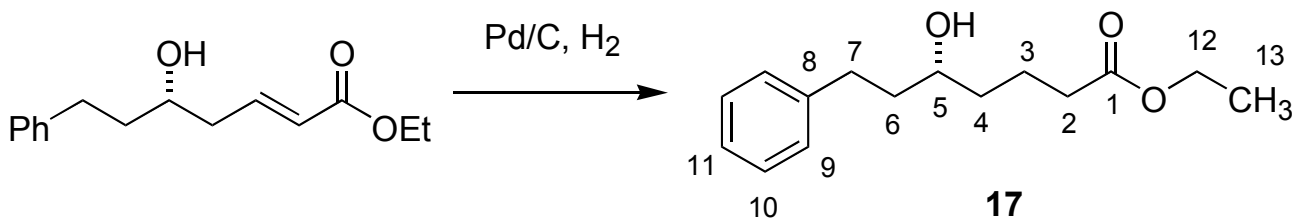

A $50 \mathrm{~mL}, 1$-neck, round-bottom flask containing a suspension of $50 \mathrm{mg}$ of $(2 E, 5 S)$ ethyl 5hydroxy-7-phenyl-2-heptenoate $(2 E, 5 S)$-14ak $(0.2 \mathrm{mmol})$ and $1.4 \mathrm{mg}$ of $10 \% \mathrm{Pd}-\mathrm{C}$ in $5 \mathrm{~mL}$ of EtOAc was stirred for $3 \mathrm{~h}$ at $23{ }^{\circ} \mathrm{C}$ under $1 \mathrm{~atm}$ of $\mathrm{H}_{2}$. After briefly evacuating the flask and venting with $\mathrm{N}_{2}$, the suspension was then filtered through Celite and the filtrate was concentrated in vacuo. The residue was purified by silica gel column chromatography (silica gel, hexanes/EtOAc, $4 / 1)$ to yield $31 \mathrm{mg}(61 \%)$ of $(5 R)-17$ as a colorless oil.

Data for $(5 R)-17$ :

${ }^{1} \mathrm{H}$ NMR: $\quad\left(400 \mathrm{MHz}, \mathrm{CDCl}_{3}\right)$

7.30-7.16 (m, $5 \mathrm{H}, \mathrm{HC}(9,10,11)), 4.12$ (q, $\left.J=7.1,2 \mathrm{H}, \mathrm{H}_{2} \mathrm{C}(12)\right), 3.61$ (m, $1 \mathrm{H}$, HC(5)), 2.77 (m, $1 \mathrm{H}, \mathrm{HC}(7)), 2.68$ (m, $1 \mathrm{H}, \mathrm{HC}(7)), 2.32$ (dt, J = 1.2, 7.56, 2 H, $\left.\mathrm{H}_{2} \mathrm{C}(2)\right), 1.79-1.67$ (m, $\left.4 \mathrm{H}, 2 \mathrm{x} \mathrm{H}_{2} \mathrm{C}(4,6)\right), 1.50$ (m, $\left.2 \mathrm{H}, \mathrm{H}_{2} \mathrm{C}(3)\right)$ ), 1.24 (t, $J=7.1$, $\left.3 \mathrm{H}, \mathrm{H}_{3} \mathrm{C}(13)\right)$

Opt. Rot.: $\quad[\alpha]_{\mathrm{D}}^{24}-6.09(\mathrm{c}=1.0, \mathrm{EtOH})$ 


\section{Preparation of (3S)-5-Phenylpentane-1,3-diol ((3S)-18) from $(2 E, 5 S)$ Ethyl 5-Hydroxy-7-} phenyl-2-heptenoate ((2E,5S)-14ak)

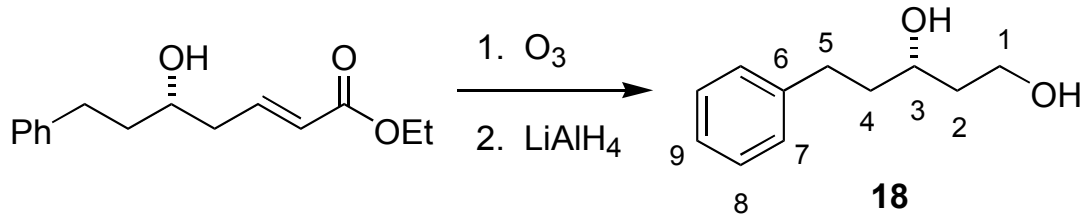

Following General Procedure 8, a solution of $93 \mathrm{mg}$ of $(2 E, 5 S)$ ethyl 5-hydroxy-7-phenyl-2heptenoate $(2 E, 5 S)$-14ak $(0.37 \mathrm{mmol})$ in $5 \mathrm{~mL}$ of pentane was treated with a solution of ozone in oxygen followed by reduction with $2.55 \mathrm{~mL}$ of a solution of $\mathrm{LiAlH}_{4}$ in THF $(0.58 \mathrm{M}, 1.48 \mathrm{mmol}$, 4 equiv) to yield, after silica gel column chromatography (EtOAc/hexanes, 2/1), $42 \mathrm{mg}$ (63\%) of $(3 S)-18$ as a colorless oil. NMR spectral data matched that previously reported in the literature. ${ }^{28}$ Data for $(3 S)-\mathbf{1 8}$ :

근 $\quad\left(400 \mathrm{MHz}, \mathrm{CDCl}_{3}\right)$

7.30-7.18 (m, 5 H, HC(7,8,9)), 3.89 (m, 2 H, $\left.\mathrm{H}_{2} \mathrm{C}(1)\right)$ ), 3.82 (m, $\left.1 \mathrm{H}, \mathrm{HC}(3)\right)$, 2.812.65 (m, $\left.2 \mathrm{H}, \mathrm{H}_{2} \mathrm{C}(5)\right), 2.60$ (s, $\left.1 \mathrm{H}, \mathrm{OH}\right), 1.85-1.70$ (m, 4 H, 2 x H $\left.\mathrm{H}_{2} \mathrm{C}(2,4)\right)$

${ }^{13} \mathrm{C} \mathrm{NMR}: \quad\left(100 \mathrm{MHz}, \mathrm{CDCl}_{3}\right)$

141.88 (C(6)), 128.43 (C(7)), 128.39 (C(8)), 125.87 (C(9)), 71.53 (C(3)), 61.72 $(\mathrm{C}(1)), 39.30(\mathrm{C}(5)), 38.25(\mathrm{C}(2)), 31.84(\mathrm{C}(4))$

Opt. Rot.: $\quad[\alpha]_{\mathrm{D}}^{24}-10.2(\mathrm{c}=1.32, \mathrm{EtOH})$, lit. $[\alpha]_{\mathrm{D}}^{24}-7.21(\mathrm{c}=1.52, \mathrm{EtOH})$ for $(3 S)-18^{28}$

TLC: $\quad R_{f} 0.26($ EtOAc/hexanes, $2 / 1)$ [p-anisaldehyde] 
Preparation of (3R)-1-Phenylpropane-1,3-diol ((3R)-16) from (2E,5R) Methyl 5-Hydroxy-2methyl-5-phenyl-2-pentenoate $((2 E, 5 R)-14 \mathrm{ba})$<smiles>COC(=O)/C(C)=C/CC(O)c1ccccc1</smiles><smiles>OCC[C@H](O)c1ccccc1</smiles>

Following General Procedure 8, a solution of $95 \mathrm{mg}$ of (2E,5R) methyl 5-hydroxy-2-methyl-5phenyl-2-pentenoate $(2 E, 5 R)$-14ba $(0.43 \mathrm{mmol})$ in $5 \mathrm{~mL}$ of pentane was treated with a solution of ozone in oxygen followed by reduction with $2.96 \mathrm{~mL}$ of a solution of $\mathrm{LiAlH}_{4}$ in THF $(0.58 \mathrm{M}$, $1.72 \mathrm{mmol}, 4$ equiv) to yield, after silica gel column chromatography (EtOAc/hexanes, 2/1), 47 mg $(71 \%)$ of $(3 R)-16$ as a colorless oil. NMR spectral data matched that previously reported in the literature. ${ }^{27}$

Data for $(3 R)-\mathbf{1 6}$ :

${ }^{1} \mathrm{H}$ NMR: $\quad\left(400 \mathrm{MHz}, \mathrm{CDCl}_{3}\right)$

7.36-7.25 (m, $5 \mathrm{H}, \mathrm{HC}(5,6,7)), 4.96$ (dd, $J=8.5,3.9,1 \mathrm{H}, \mathrm{HC}(3))$ ), 3.86 (t, $J=5.6$, $\left.2 \mathrm{H}, \mathrm{H}_{2} \mathrm{C}(1)\right), 2.55$ (s, $\left.1 \mathrm{H}, \mathrm{OH}\right), 2.1-1.85$ (m, $\left.2 \mathrm{H}, \mathrm{H}_{2} \mathrm{C}(2)\right)$

Opt. Rot.: $\quad[\alpha]_{\mathrm{D}}^{24}+57.1\left(\mathrm{c}=0.978, \mathrm{CHCl}_{3}\right)$, lit. $[\alpha]_{\mathrm{D}}^{24}-63.0\left(\mathrm{c}=0.958, \mathrm{CHCl}_{3}\right)$ for $(3 S)-\mathbf{1 6}^{27}$

\section{Preparation of (4R)-4-(Acetyloxy)-4-phenyl-2-butanone ((4R)-19) from (2E,5R) Ethyl 5-} Hydroxy-3-methyl-5-phenyl-2-pentenoate $((2 E, 5 R)-14 \mathrm{ca})$

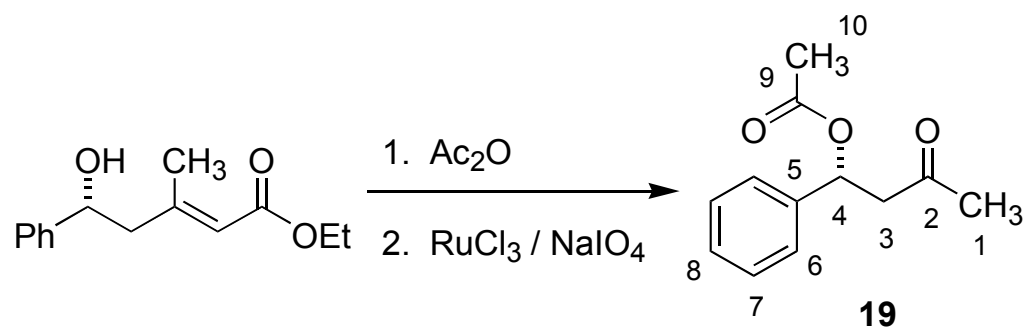

To a $25 \mathrm{~mL}$, 1-neck, round-bottom flask containing a solution of $53 \mathrm{mg}$ of $(2 E, 5 R)$ ethyl 5 hydroxy-3-methyl-5-phenyl-2-pentenoate $((2 E, 5 R)-14 \mathrm{ca})(0.23 \mathrm{mmol})$ in $0.5 \mathrm{~mL}$ of acetic anhydride ( $5.3 \mathrm{mmol}, 23$ equiv) was slowly added $0.5 \mathrm{~mL}$ of pyridine ( $6.2 \mathrm{mmol}, 27$ equiv). The solution was allowed to stir at room temperature for $90 \mathrm{~min}$ before the addition of $10 \mathrm{~mL}$ of $\mathrm{Et}_{2} \mathrm{O}$. The cloudy solution was then washed with $0.1 \mathrm{M} \mathrm{HCl}(20 \mathrm{~mL})$ followed by washing with 
brine $(20 \mathrm{~mL})$. The organic layer was then separated and dried over $\mathrm{Na}_{2} \mathrm{SO}_{4}(5 \mathrm{~g})$, filtered and the filtrate was concentrated in vacuo. The residue was then transferred to a $25 \mathrm{~mL}$, 1-neck flask and dissolved in a mixture of $2 \mathrm{~mL} \mathrm{CH}_{2} \mathrm{Cl}_{2}, 2 \mathrm{~mL} \mathrm{CH} \mathrm{CH}_{3} \mathrm{Cnd} 2 \mathrm{~mL} \mathrm{H}_{2} \mathrm{O}$. To this solution was added $1 \mathrm{mg}$ of $\mathrm{RuCl}_{3}-\mathrm{H}_{2} \mathrm{O}$ (0.0046 mmol, 0.02 equiv) and $206 \mathrm{mg}$ of $\mathrm{NaIO}_{4}(0.96 \mathrm{mmol}, 4.2$ equiv). The biphasic mixture was stirred at room temperature for $2 \mathrm{~h}$ prior to addition of $10 \mathrm{~mL}$ $\mathrm{M} \mathrm{HCl}$. The solution was then extracted with $\mathrm{CH}_{2} \mathrm{Cl}_{2}(2 \times 10 \mathrm{~mL})$. The combined organic layers were washed with brine $(10 \mathrm{~mL})$, dried over $\mathrm{Na}_{2} \mathrm{SO}_{4}(5 \mathrm{~g})$, filtered and the filtrate was concentrated in vacuo. The residue was purified by silica gel column chromatography (hexanes/EtOAc, 4/1) to yield $28 \mathrm{mg}(55 \%)$ of $(4 R)-19$ as a colorless oil. NMR spectral data matched that previously reported in the literature. ${ }^{29}$

Data for $(4 R)-19$ :

1 NM: $\quad\left(400 \mathrm{MHz}, \mathrm{CDCl}_{3}\right)$

7.34-7.29 (m, 5 H, HC(6,7,8)), 6.18 (dd, $J=8.5,4.9,1 \mathrm{H}, \mathrm{HC}(4)), 3.11$ (dd, $J=$ 16.6, 8.8, $1 \mathrm{H}, \mathrm{HC}(3)), 2.82(\mathrm{dd}, J=16.6,4.9,1 \mathrm{H}, \mathrm{HC}(3))$, 2.14 (s, $\left.3 \mathrm{H}, \mathrm{H}_{3} \mathrm{C}(1)\right)$, $2.03\left(\mathrm{~s}, 3 \mathrm{H}, \mathrm{H}_{3} \mathrm{C}(10)\right)$

${ }^{13} \mathrm{C} \mathrm{NMR}: \quad\left(100 \mathrm{MHz}, \mathrm{CDCl}_{3}\right)$

204.61 (C(2)), 169.77 (C(9)), 139.62 (C(5)), 128.58 (C(7)), 128.21 (C(6)), 126.39 (C(8)), 71.54 (C(4)), 49.80 (C(3)), 30.40 (C(10)), 21.03 (C(1))

Opt. Rot.: $\quad[\alpha]_{\mathrm{D}}^{24}+60.0\left(\mathrm{c}=0.71, \mathrm{CHCl}_{3}\right)$, lit. $[\alpha]_{\mathrm{D}}^{24}+64.6\left(\mathrm{c}=0.71, \mathrm{CHCl}_{3}\right)$ for $(4 R)-19^{29}$ TLC: $\quad R_{f} 0.35$ (hexanes/EtOAc, 4/1) [UV(254)/ $\left.\mathrm{KMnO}_{4}\right]$

Preparation of $(2 R, 3 R)-2-M e t h y l-1-p h e n y l p r o p a n e-1,3-d i o l ~((2 R, 3 R)-20)$ from $(2 E, 4 R, 5 R)$ tert-Butyl 5-Hydroxy-4-methyl-5-phenyl-2-pentenoate $((2 E, 4 R, 5 R)-14 \mathrm{da})$

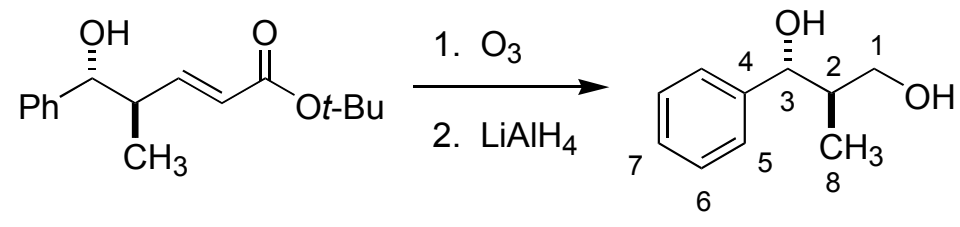

20

Following General Procedure 8, a solution of $95 \mathrm{mg}$ of $(2 E, 4 R, 5 R)$ tert-butyl 5-hydroxy-4methyl-5-phenyl-2-pentenoate $(2 E, 4 R, 5 R)$-14da $(0.36 \mathrm{mmol})$ in $5 \mathrm{~mL}$ of pentane was treated 
with a solution of ozone in oxygen followed by reduction with $2.48 \mathrm{~mL}$ a solution of $\mathrm{LiAlH}_{4}$ in THF (0.58 M, 1.44 mmol, 4 equiv) to yield, after silica gel column chromatography (EtOAc/hexanes, 2/1), $26 \mathrm{mg}$ (44\%) of ( $2 R, 3 R)-20$ as a colorless oil. NMR spectral data matched that previous reported in the literature. ${ }^{27}$

Data for $(2 R, 3 R)-\mathbf{2 0}$ :

${ }^{1} \mathrm{H}$ NMR: $\quad\left(400 \mathrm{MHz}, \mathrm{CDCl}_{3}\right)$

7.37-7.26 (m, $5 \mathrm{H}, \mathrm{HC}(5,6,7)), 4.50(\mathrm{~d}, J=8.5,1 \mathrm{H}, \mathrm{HC}(3)), 3.70(\mathrm{~m}, 2 \mathrm{H}$, $\mathrm{H}_{2} \mathrm{C}(1)$ ), 3.19 (s, $\left.2 \mathrm{H}, \mathrm{OH}\right), 2.03$ (m, $\left.1 \mathrm{H}, \mathrm{H}_{2} \mathrm{C}(2)\right), 0.67$ (d, $J=7.1,3 \mathrm{H}, \mathrm{H}_{3} \mathrm{C}(8)$ )

${ }^{13} \mathrm{C}$ NMR: $\quad\left(100 \mathrm{MHz}, \mathrm{CDCl}_{3}\right)$

$143.29(\mathrm{C}(4)), 128.39(\mathrm{C}(6)), 127.80(\mathrm{C}(5)), 126.66(\mathrm{C}(7)), 80.83(\mathrm{C}(3)), 67.92$ $(\mathrm{C}(1)), 41.51(\mathrm{C}(2)), 13.75(\mathrm{C}(8))$

Opt. Rot.: $\quad[\alpha]_{\mathrm{D}}^{24}+35.17\left(\mathrm{c}=0.34, \mathrm{CHCl}_{3}\right)$, lit. $[\alpha]_{\mathrm{D}}^{24}-46.8\left(\mathrm{c}=0.35, \mathrm{CHCl}_{3}\right)$ for $(2 S, 3 S)-\mathbf{2 0} \mathrm{o}^{27}$

TLC: $\quad R_{f} 0.47$ (EtOAc/hexanes, 2/1) [p-anisaldehyde]

\section{Preparation of ( $\alpha$-Chlorocyclohexylmethoxy)trichlorosilane (23)}

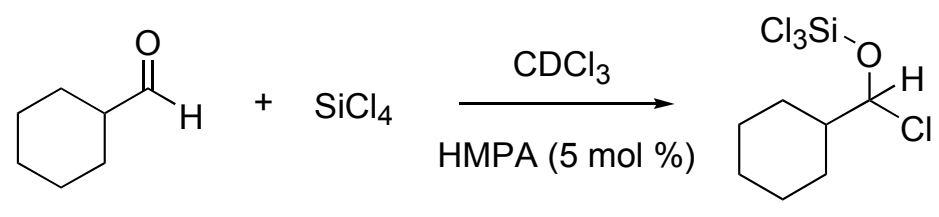

Into a dry NMR tube was added $30 \mu \mathrm{L} \quad(0.25 \mathrm{mmol})$ of freshly distilled cyclohexanecarboxaldehyde $\mathbf{5 j}$ and $1.0 \mathrm{~mL}$ of $\mathrm{CDCl}_{3}$ at room temperature under nitrogen. To this solution was added $2.2 \mu \mathrm{L}$ of ( $0.0125 \mathrm{mmol}, 0.05$ equiv) of HMPA followed by $34 \mu \mathrm{L}(0.3$ mmol, 1.2 equiv) of $\mathrm{SiCl}_{4}$. The NMR tube was vortexed for $1 \mathrm{~min}$. to insure mixing. The tube was vortexed and then inserted NMR probe and the ${ }^{1} \mathrm{H}$ NMR spectrum was acquired at room temperature. After $24 \mathrm{~h}$ at room temerpature, a ${ }^{13} \mathrm{C}$ NMR was acquired.

\section{Data for 23:}

${ }^{1} \mathrm{H}$ NMR: $\quad\left(500 \mathrm{MHz}, \mathrm{CDCl}_{3}\right)$

$5.78(\mathrm{~d}, J=4.9,1 \mathrm{H}, \mathrm{HC}(1)), 1.94-1.65$ (m, $6 \mathrm{H}, 6$ x H(Cy)), 1.30-1.10 (m, 5 H, 5 $\mathrm{x} \mathrm{H}(\mathrm{Cy}))$ 
${ }^{13} \mathrm{C} \mathrm{NMR}: \quad\left(126 \mathrm{MHz}, \mathrm{CDCl}_{3}\right)$

92.95 (C(1)), 47.05 (C(2)), 28.03 (C(3)), 27.56 (C(7)), 26.19 (C(6)), 25.73 (C(4)), $25.63(\mathrm{C}(5))$

Reaction of Crotonaldehyde with $\mathrm{SiCl}_{4}$ in the presence of HMPA

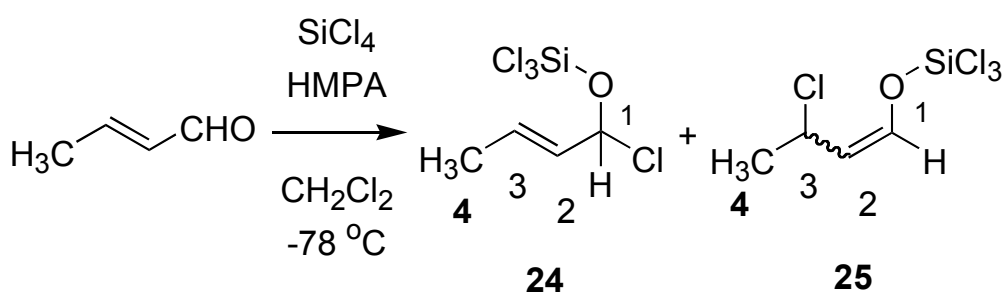

Into an oven dried $5 \mathrm{~mm}$ NMR tube, $17 \mu \mathrm{L}$ of crotonaldehyde $(\mathbf{5 m})(0.2 \mathrm{mmol})$ and $3 \mu \mathrm{L}$ of HMPA (0.02 mmol, 0.1 equiv) was dissolved in $0.8 \mathrm{~mL}$ of $\mathrm{CDCl}_{3}$. The tube was cooled in a dry ice / isopropanol bath and $25 \mu \mathrm{L}$ of $\mathrm{SiCl}_{4}(0.22 \mathrm{mmol}, 1.1$ equiv) was added. The tube was vortexed and then inserted into a pre-cooled NMR probe at $-70{ }^{\circ} \mathrm{C}$. The ${ }^{1} \mathrm{H}$ NMR spectrum was acquired at $-70{ }^{\circ} \mathrm{C}$. A mixture of species was observed: $42 \%$ 5m / 27\% $\gamma-\mathrm{Cl} 24 / 27 \% \alpha-\mathrm{Cl} 25$.

Data for 24:

${ }^{1} \mathrm{H}$ NMR: $\quad\left(500 \mathrm{MHz}, \mathrm{CDCl}_{3}\right)$

$6.54(\mathrm{~d}, J=12.0,1 \mathrm{H}, \mathrm{HC}(1)), 5.51(\mathrm{dd}, J=12.0,9.8,1 \mathrm{H}, \mathrm{HC}(2)), 4.54$ (dq, $J=$

9.6, 6.6, $1 \mathrm{H}, \mathrm{HC}(3)), 1.53\left(\mathrm{~d}, J=6.6,3.4, \mathrm{H}_{3} \mathrm{C}(4)\right)$

Data for 25:

${ }^{1} \mathrm{H}$ NMR: $\quad\left(500 \mathrm{MHz}, \mathrm{CDCl}_{3}\right)$

$6.25(\mathrm{~d}, J=5.6,1 \mathrm{H}, \mathrm{HC}(1)), 5.08(\mathrm{dd}, J=15.7,5.4,1 \mathrm{H}, \mathrm{HC}(2)), 5.02(\mathrm{~m}, 1 \mathrm{H}$, $\mathrm{HC}(3)), 1.49\left(\mathrm{~d}, J=6.4,3 \mathrm{H}, \mathrm{H}_{3} \mathrm{C}(4)\right)$ 


\section{Calculated Structure of the Aldehyde-Silyl cation Complex (Figure 11)}

Model created using the PC version of GAMESS(US) QC package at the PM3 level. ${ }^{30}$ Stucture visulaized using Chem3D with some hydrogens omitted for clarity.

\begin{tabular}{|c|c|c|c|c|}
\hline \multicolumn{5}{|c|}{$\begin{array}{l}\text { RESULTS FROM SUCCESSFUL RHF GEOMETRY SEARCH } \\
\text { COORDS, ORBS, GRADIENT, AND APPROX. HESSIAN } \\
\text { COORDINATES OF SYMMETRY UNIQUE ATOMS (ANGS) }\end{array}$} \\
\hline Atom & Charge & $\mathrm{X}$ & $\mathrm{Y}$ & $\mathrm{Z}$ \\
\hline $\mathrm{C}$ & 6 & 7.08576704 & -4.5424505 & 1.57103129 \\
\hline $\mathrm{C}$ & 6 & 6.08218144 & -4.0270331 & 2.34543123 \\
\hline $\mathrm{C}$ & 6 & 7.58284018 & -3.8025013 & 0.4749762 \\
\hline $\mathrm{N}$ & 7 & 3.09338375 & 2.87554011 & -0.9171932 \\
\hline $\mathrm{C}$ & 6 & 4.38728795 & 3.56600712 & -0.7167774 \\
\hline $\mathrm{C}$ & 6 & 7.08350719 & -2.5599204 & 0.19138778 \\
\hline $\mathrm{C}$ & 6 & 1.92993776 & 3.7958594 & -0.9008909 \\
\hline $\mathrm{C}$ & 6 & -7.2085942 & -3.4755011 & -2.1077765 \\
\hline $\mathrm{C}$ & 6 & 6.05483553 & -1.9853507 & 0.98920839 \\
\hline $\mathrm{C}$ & 6 & -6.1601344 & -2.956205 & -2.8182928 \\
\hline $\mathrm{C}$ & 6 & 5.5376727 & -2.7467525 & 2.05815336 \\
\hline $\mathrm{C}$ & 6 & -7.6321253 & -2.8499385 & -0.9136188 \\
\hline $\mathrm{C}$ & 6 & 4.44903291 & -2.2436369 & 2.81991553 \\
\hline $\mathrm{C}$ & 6 & 3.89064544 & -1.0383337 & 2.51255091 \\
\hline $\mathrm{N}$ & 7 & -2.3332423 & 2.99282974 & 1.34782975 \\
\hline $\mathrm{C}$ & 6 & 4.42341075 & -0.2434609 & 1.45625281 \\
\hline $\mathrm{N}$ & 7 & 3.8325934 & 1.05664464 & 1.17831691 \\
\hline $\mathrm{P}$ & 15 & 2.98423692 & 1.19529317 & -0.369194 \\
\hline $\mathrm{C}$ & 6 & 3.14900452 & 1.69617355 & 2.32357157 \\
\hline
\end{tabular}




\begin{tabular}{|c|c|c|c|c|}
\hline $\mathrm{C}$ & 6 & 5.51289579 & -0.6842203 & 0.71804805 \\
\hline $\mathrm{C}$ & 6 & 6.0835264 & 0.11188875 & -0.3844739 \\
\hline $\mathrm{C}$ & 6 & 5.31651495 & 0.46432015 & -1.4871189 \\
\hline $\mathrm{C}$ & 6 & 5.92328216 & 1.05520637 & -2.6348737 \\
\hline $\mathrm{C}$ & 6 & 7.24848938 & 1.37708094 & -2.6267067 \\
\hline $\mathrm{C}$ & 6 & 8.02977845 & 1.16572693 & -1.4572445 \\
\hline $\mathrm{C}$ & 6 & 7.45173738 & 0.53917565 & -0.332999 \\
\hline $\mathrm{N}$ & 7 & 3.8915398 & 0.19019543 & -1.4901711 \\
\hline $\mathrm{C}$ & 6 & 3.28534912 & -0.1521728 & -2.7909756 \\
\hline $\mathrm{C}$ & 6 & 8.24509409 & 0.37740293 & 0.83604997 \\
\hline $\mathrm{C}$ & 6 & 9.54559151 & 0.80514202 & 0.8640058 \\
\hline $\mathrm{C}$ & 6 & 10.1256877 & 1.41077288 & -0.2729728 \\
\hline $\mathrm{C}$ & 6 & 9.38480434 & 1.58773717 & -1.4106807 \\
\hline $\mathrm{O}$ & 8 & 1.43829019 & 0.8459831 & -0.2343668 \\
\hline $\mathrm{C}$ & 6 & -3.5073538 & 3.89364582 & 1.34410533 \\
\hline $\mathrm{C}$ & 6 & -7.0111372 & -1.7152737 & -0.4653716 \\
\hline $\mathrm{C}$ & 6 & -1.0291729 & 3.70053866 & 1.37850526 \\
\hline $\mathrm{C}$ & 6 & -5.9293722 & -1.1395062 & -1.1886361 \\
\hline $\mathrm{C}$ & 6 & -5.4942699 & -1.7855505 & -2.3648527 \\
\hline $\mathrm{C}$ & 6 & -4.3731716 & -1.2772519 & -3.0754057 \\
\hline $\mathrm{C}$ & 6 & -3.7023782 & -0.1830386 & -2.614299 \\
\hline $\mathrm{C}$ & 6 & -4.1386783 & 0.48869015 & -1.4353189 \\
\hline $\mathrm{N}$ & 7 & -3.4203128 & 1.66442233 & -0.9709396 \\
\hline $\mathrm{P}$ & 15 & -2.5163114 & 1.43970645 & 0.53509634 \\
\hline $\mathrm{C}$ & 6 & -2.7179272 & 2.43939767 & -2.0178926 \\
\hline $\mathrm{C}$ & 6 & -5.2582695 & 0.04797492 & -0.7436125 \\
\hline $\mathrm{C}$ & 6 & -5.7350767 & 0.73251409 & 0.47215967 \\
\hline $\mathrm{C}$ & 6 & -4.9267832 & 0.84520687 & 1.59538953 \\
\hline $\mathrm{C}$ & 6 & -5.4548627 & 1.35797418 & 2.81693834 \\
\hline
\end{tabular}




\begin{tabular}{|c|c|c|c|c|}
\hline $\mathrm{C}$ & 6 & -6.7348392 & 1.82568815 & 2.87587216 \\
\hline $\mathrm{C}$ & 6 & -7.5448992 & 1.84770351 & 1.70775565 \\
\hline $\mathrm{C}$ & 6 & -7.047356 & 1.31001446 & 0.50198775 \\
\hline $\mathrm{N}$ & 7 & -3.5426296 & 0.4026807 & 1.53451516 \\
\hline $\mathrm{C}$ & 6 & -2.9660842 & -0.1082445 & 2.7946377 \\
\hline $\mathrm{C}$ & 6 & -7.8610444 & 1.38839779 & -0.6617041 \\
\hline $\mathrm{C}$ & 6 & -9.1062855 & 1.95555341 & -0.608108 \\
\hline $\mathrm{C}$ & 6 & -9.6088062 & 2.46920802 & 0.60844805 \\
\hline $\mathrm{C}$ & 6 & -8.8452813 & 2.41718157 & 1.7437368 \\
\hline $\mathrm{O}$ & 8 & -1.1077169 & 0.78398494 & 0.27987444 \\
\hline $\mathrm{C}$ & 6 & 1.77335343 & 4.61231188 & 0.38236941 \\
\hline $\mathrm{C}$ & 6 & 0.4974222 & 5.43758389 & 0.35940293 \\
\hline $\mathrm{C}$ & 6 & -0.7624381 & 4.60701199 & 0.17756687 \\
\hline $\mathrm{Si}$ & 14 & 0.16093784 & -0.2901569 & -0.1590629 \\
\hline $\mathrm{Cl}$ & 17 & 1.65015594 & -1.9801939 & -0.6569166 \\
\hline $\mathrm{C}$ & 6 & -3.8744603 & -4.7385572 & -0.7390823 \\
\hline $\mathrm{C}$ & 6 & -2.9764927 & -3.6869722 & -0.5995046 \\
\hline $\mathrm{C}$ & 6 & -3.6753199 & -5.9271124 & -0.0442317 \\
\hline $\mathrm{C}$ & 6 & -2.5728252 & -6.0682579 & 0.79672271 \\
\hline $\mathrm{C}$ & 6 & -1.6722593 & -5.0234167 & 0.94444001 \\
\hline $\mathrm{C}$ & 6 & -1.8708786 & -3.8212407 & 0.24794536 \\
\hline $\mathrm{C}$ & 6 & -0.882289 & -2.7613019 & 0.43127183 \\
\hline $\mathrm{O}$ & 8 & -1.0141064 & -1.6489997 & -0.1172918 \\
\hline $\mathrm{Cl}$ & 17 & -0.332367 & 0.01412551 & -2.3465979 \\
\hline $\mathrm{H}$ & 1 & 7.50778321 & -5.5297699 & 1.78662992 \\
\hline $\mathrm{H}$ & 1 & 5.67937416 & -4.5947499 & 3.19217981 \\
\hline $\mathrm{H}$ & 1 & 8.37324461 & -4.2365027 & -0.1469472 \\
\hline $\mathrm{H}$ & 1 & 4.55258745 & 3.8781661 & 0.32622329 \\
\hline $\mathrm{H}$ & 1 & 4.42363112 & 4.46553184 & -1.3481828 \\
\hline
\end{tabular}




\begin{tabular}{|c|c|c|c|c|}
\hline $\mathrm{H}$ & 1 & 5.20963442 & 2.90316721 & -1.0243493 \\
\hline $\mathrm{H}$ & 1 & 7.47459334 & -1.9909497 & -0.6616107 \\
\hline $\mathrm{H}$ & 1 & 2.05317413 & 4.47800962 & -1.7683671 \\
\hline $\mathrm{H}$ & 1 & 1.00095639 & 3.21225118 & -1.096765 \\
\hline $\mathrm{H}$ & 1 & -7.7291413 & -4.3743424 & -2.4559593 \\
\hline $\mathrm{H}$ & 1 & -5.8208021 & -3.4311596 & -3.7467341 \\
\hline $\mathrm{H}$ & 1 & -8.4670763 & -3.283 & -0.3517147 \\
\hline $\mathrm{H}$ & 1 & 4.04781548 & -2.850567 & 3.64008225 \\
\hline $\mathrm{H}$ & 1 & 2.99322169 & -0.683301 & 3.05202645 \\
\hline $\mathrm{H}$ & 1 & 2.63456527 & 2.61100289 & 1.9815884 \\
\hline $\mathrm{H}$ & 1 & 3.9060297 & 1.97989419 & 3.06657863 \\
\hline $\mathrm{H}$ & 1 & 2.41246464 & 1.02687348 & 2.81255911 \\
\hline $\mathrm{H}$ & 1 & 5.30397866 & 1.22505132 & -3.5307543 \\
\hline $\mathrm{H}$ & 1 & 7.72453902 & 1.81397805 & -3.5126961 \\
\hline $\mathrm{H}$ & 1 & 3.61540486 & -1.1661222 & -3.0568319 \\
\hline $\mathrm{H}$ & 1 & 3.596459 & 0.53593268 & -3.5967975 \\
\hline $\mathrm{H}$ & 1 & 2.18561167 & -0.1655842 & -2.7018778 \\
\hline $\mathrm{H}$ & 1 & 7.79608535 & -0.1030063 & 1.71501439 \\
\hline $\mathrm{H}$ & 1 & 10.1504283 & 0.67928963 & 1.76873653 \\
\hline $\mathrm{H}$ & 1 & 11.1708397 & 1.73620315 & -0.2316536 \\
\hline $\mathrm{H}$ & 1 & 9.82356374 & 2.05842189 & -2.2983242 \\
\hline $\mathrm{H}$ & 1 & -3.6856804 & 4.37236442 & 0.36839182 \\
\hline $\mathrm{H}$ & 1 & -3.3592422 & 4.68909769 & 2.08851946 \\
\hline $\mathrm{H}$ & 1 & -4.4041629 & 3.32386687 & 1.6300928 \\
\hline $\mathrm{H}$ & 1 & -7.3478444 & -1.2304918 & 0.46016825 \\
\hline $\mathrm{H}$ & 1 & -1.0136067 & 4.29529785 & 2.31608622 \\
\hline $\mathrm{H}$ & 1 & -0.2358062 & 2.93070665 & 1.48008196 \\
\hline $\mathrm{H}$ & 1 & -4.0460068 & -1.7865202 & -3.9902654 \\
\hline $\mathrm{H}$ & 1 & -2.7934601 & 0.17692827 & -3.1335076 \\
\hline
\end{tabular}




\begin{tabular}{|c|c|c|c|c|}
\hline $\mathrm{H}$ & 1 & -2.0340438 & 3.16841843 & -1.546832 \\
\hline $\mathrm{H}$ & 1 & -3.4673021 & 2.98761173 & -2.6036034 \\
\hline $\mathrm{H}$ & 1 & -2.1303043 & 1.80000029 & -2.7073704 \\
\hline $\mathrm{H}$ & 1 & -4.817367 & 1.3545742 & 3.71605523 \\
\hline $\mathrm{H}$ & 1 & -7.1496912 & 2.20384779 & 3.81816037 \\
\hline $\mathrm{H}$ & 1 & -3.3865838 & -1.1061538 & 2.97951041 \\
\hline $\mathrm{H}$ & 1 & -3.2098129 & 0.52479694 & 3.66693259 \\
\hline $\mathrm{H}$ & 1 & -1.8701298 & -0.199569 & 2.70408964 \\
\hline $\mathrm{H}$ & 1 & -7.4736197 & 0.9826044 & -1.6051449 \\
\hline $\mathrm{H}$ & 1 & -9.7264919 & 2.01544693 & -1.5093816 \\
\hline $\mathrm{H}$ & 1 & -10.611732 & 2.90968257 & 0.63021091 \\
\hline $\mathrm{H}$ & 1 & -9.2228027 & 2.81612361 & 2.69270944 \\
\hline $\mathrm{H}$ & 1 & 2.63885458 & 5.2936438 & 0.51565653 \\
\hline $\mathrm{H}$ & 1 & 1.78665575 & 3.94558337 & 1.2763146 \\
\hline $\mathrm{H}$ & 1 & 0.5566113 & 6.18181015 & -0.4606421 \\
\hline $\mathrm{H}$ & 1 & 0.42716934 & 6.02861665 & 1.29479141 \\
\hline $\mathrm{H}$ & 1 & -0.6775895 & 3.99348215 & -0.7551649 \\
\hline $\mathrm{H}$ & 1 & -1.6181704 & 5.29299007 & 0.01034665 \\
\hline $\mathrm{H}$ & 1 & -4.7455983 & -4.618108 & -1.4001566 \\
\hline $\mathrm{H}$ & 1 & -3.1433686 & -2.7556586 & -1.1618859 \\
\hline $\mathrm{H}$ & 1 & -4.3849028 & -6.7545854 & -0.159032 \\
\hline $\mathrm{H}$ & 1 & -2.4153423 & -7.0071483 & 1.33998442 \\
\hline $\mathrm{H}$ & 1 & -0.801655 & -5.1390102 & 1.60289515 \\
\hline $\mathrm{H}$ & 1 & 0.00262044 & -2.9457676 & 1.07499146 \\
\hline $\mathrm{Cl}$ & 17 & 0.50006703 & -0.603103 & 2.12263458 \\
\hline
\end{tabular}




\section{References}

(1) Commercially available 1-naphthaldehyde is contaminated by $2-4 \%$ 2naphthaldehyde. This isomeric aldehyde cannot be removed by distillation.

(2) Denmark, S.E.; Wynn T. J. Am Chem. Soc. 2001, 123, 6199.

(3) Journet, M., Cai, D., DiMichele, L.M., Larsen, R.D. Tetrahedron Lett. 1998, 39, 6427.

(4) Ireland, R.E., Wipf, P., Armstrong, J.D. J. Org. Chem. 1991 56, 650.

(5) McDougal, P.G., Rico, J.G., Oh, Y-I., Condon, B.D. J. Org. Chem. 1986 51, 3388.

(6) Shimojo, M., Matsumoto, K., Hatanaka, M. Tetrahedron 2000, 56, 9281.

(7) Yamaguchi, M., Takata, T., Endo, T. J. Org. Chem. 1990, 55, 1490.

(8) Wilcox, C.S.; Babston, R.E. J. Org. Chem. 1984, 49, 1451.

(9) Heathcock, C.H., Davidsen, S.K., Hug, K.T., Flippin, L.A. J. Org. Chem. 1986, 51, 3027.

(10) Hertler, W. R.; Reddy, G. S.; Sogah, D. Y. J. Org. Chem. 1988, 53, 3532.

(11) Hine, J.; Kanagasabapathy, V. M. J. Org. Chem. 1982, 47, 2745.

(12) Lutz, G.P.; Du, H.; Gallagher, D.J.; Beak, P. J. Org. Chem. 1996, 61, 4542.

(13) Carreira, E.M.; Singer R.A.; Lee, W. J. Am. Chem .Soc. 1994, 116, 8837.

(14) Denmark, S.E.; Winter, S.B.D.; Su, X.; Wong, K.-T. J. Am. Chem. Soc. 1996, 118, 7404.

(15) Fujitsawa, T.; Fujimura, A.; Sato, T. Bull. Chem. Soc. Jpn. 1988, 61, 1273.

(16) Curran, D. P., Scanga, S. A., Fenk, C. J. J. Org. Chem. 1984, 49, 3474.

(17) Ucello-Barretta, G., Pini, D., Mastantuono, A., Salvadori, P. Tetrahedron: Asym. 1995, 6, 1965.

(18) To simplify CSP-SFC analysis, the 2-naphthaldehyde had to be removed from the commericially available 1-naphthaldehyde prior to use. To do this, ethanol was added to a freshly made solution of saturated aqueous sodium bisulfite until the solution became cloudy. Water was then added until the solution was clear. The 1-naphthaldehyde was then added to this stirred solution and after $15 \mathrm{~min}$, the bisulfite salt was isolated by filtration. The resulting white solid could then be recrystallized from EtOH to yield the isomerically pure, 1-naphthaldehyde bisulfite salt. The 1-naphthaldehyde could be recoverd by dissolving the bisulfite salt in a small 
amount of water and adding an equal volume of a saturated aqueous solution of $\mathrm{NaHCO}_{3}$. The resulting soution was extracted with $\mathrm{CH}_{2} \mathrm{Cl}_{2}$, the combined organic layers were dried over $\mathrm{K}_{2} \mathrm{CO}_{3}$ and concentrated in vacuo to provide the isomerically pure 1-naphthaldehyde.

(19) (a) Kamimura, A., Mitsudera, H., Asano, S., Kidera, S., Kakehi, A. J. Org. Chem. 1999, 64, 6353. (b) Corey, E.J., Kim, S.S. J. Am. Chem. Soc. 1990, 117, 4976.

(20) Kiyooka, S-I., Shimizu, A., Torii, S. Tetrahedron Lett. 1998, 39, 5237.

(21) Data obtained from a chromatographically homogeneous sample.

(22) Kobayashi, S.; Ishitani, H.; Yamashita, Y.; Ueno, M.; Shimizu, H. Tetrahedron 2001, 57,861 .

(23) Hunter, T. J.; O'Doherty, G. A. Org. Lett. 2001, 3, 1049.

(24) Sumida, S.; Ohga, M.; Mitani, J.; Nokami, J. J. Am. Chem. Soc. 2000, 122, 1310.

(25) Dugger, R. W.; Heathcock, C. H. J. Org. Chem. 1980, 45, 1181.

(26) Singer, R. A.; Carriera, E. M. J. Am. Chem. Soc. 1995, 117, 12360.

(27) Masamune, S.; Sato, T.; Kim, B.; Wollman, T.A. J. Am. Chem. Soc. 1986, 108, 8279 .

(28) Nuñez, M. T.; Martin, V. S. J. Org. Chem. 1990, 55, 1928.

(29) Nair, M. S.; Joly, S. Tetrahedron: Asymmetry 2000, 11, 2049.

(30) (a) from http://classic.chem.msu.su/gran/gamess/index.html by Granovsky, A. A. (b) Schmidt, M. W.; Baldridge, K. K.; Boatz, J. A.; Elbert, S. T.; Gordon, M. S.; Jensen, J. J.; Matsunaga, N.; Nguyen, K. A.; Su, S.; Windus, T. L.; Dupuis, M.; Montgomery, J. A. J. Comput. Chem. 1993, 14, 1347. 